\title{
Nuclear Resonance Fluorescence to Measure Plutonium Mass in Spent Nuclear Fuel
}

\author{
Prepared for: \\ Office of Dismantlement and Transparency (NA-241) \\ U.S. Department of Energy \\ Prepared by: \\ B.A. Ludewigt ${ }^{1}$, B.J. Quiter ${ }^{1}$, and S.D. Ambers ${ }^{2}$ \\ ${ }^{1}$ Lawrence Berkeley National Laboratory, Berkeley, CA 94720 \\ ${ }^{2}$ University of California, Berkeley, CA 94720
}

January 14, 2011 


\section{DISCLAIMER}

This document was prepared as an account of work sponsored by the United States Government. While this document is believed to contain correct information, neither the United States Government nor any agency thereof, nor The Regents of the University of California, nor any of their employees, makes any warranty, express or implied, or assumes any legal responsibility for the accuracy, completeness, or usefulness of any information, apparatus, product, or process disclosed, or represents that its use would not infringe privately owned rights. Reference herein to any specific commercial product, process, or service by its trade name, trademark, manufacturer, or otherwise, does not necessarily constitute or imply its endorsement, recommendation, or favoring by the United States Government or any agency thereof, or The Regents of the University of California. The views and opinions of authors expressed herein do not necessarily state or reflect those of the United States Government or any agency thereof or The Regents of the University of California.

\section{ACKNOWLEDGMENTS}

This work was supported by the Next Generation Safeguards Initiative (NGSI), Office of Nonproliferation and International Security (NA-24), National Nuclear Security Administration, U.S. Department of Energy under Contract No. DE-AC02-05CH11231. 


\section{Executive Summary}

The Next Generation Safeguard Initiative (NGSI) of the U.S. Department of Energy is supporting a multi-lab/university collaboration to quantify the plutonium $(\mathrm{Pu})$ mass in spent nuclear fuel (SNF) assemblies and to detect the diversion of pins with non-destructive assay (NDA) methods. Nuclear Resonance Fluorescence (NRF) has been investigated as one of 14 NDA techniques included in the NGSI study. In contrast to many of the other, older techniques, NRF had not previously been considered for nuclear safeguards or similar applications and basic aspects including nuclear data, modeling and simulation tools, measurement methods, and instrumentation needed to be researched.

Nuclear resonance fluorescence is the process by which a nucleus is excited to a specific state by the absorption of a photon, then subsequently de-excites to the ground state by the emission of one or more $\gamma$ rays. The energies of the $\gamma$ rays are characteristic of the specific state that underwent NRF and therefore are characteristic of the isotope. The emitted NRF $\gamma$ rays are detected at backwards angles where the backgrounds from elastically and inelastically scattered photons are lowest. Two distinct assay methods, the backscatter and the transmission methodsare analyzed in this report. In both cases a beam of energetic photons excites nuclear resonances of the isotopes of interest (IOI) within the SNF. In the backscatter assay geometry, the emitted $\mathrm{NRF} \gamma$ rays detected at backwards angles are a measure of the amount of a specific isotope in the SNF. In transmission assay, a detection system down-stream of the SNF measures the excess attenuation of resonant-energy photons due to NRF in the SNF assembly. The detection system consists of a thin sheet composed of the isotope of interest, called the transmission detector (TD), and a detector array that measures the NRF $\gamma$ rays emanating from the TD. Given the low concentrations of the $\mathrm{Pu}$ isotopes in SNF and the relatively small integrated nuclear resonance cross sections, the main challenge for achieving precise and accurate measurements lies in accruing sufficient counting statistics in an acceptable measurement time.

The goals of this study were to design and model suitable NRF measurement methods, to quantify capabilities and corresponding instrumentation requirements, to investigate possible implementations, and to evaluate the prospects and potential of the technique for the assay of SNF assemblies, i.e., for the measurement of their Pu isotopic content.Analytical models were developed that enabled calculation of NRF signals for a variety of backscatter and transmission geometries. Extensive simulations were performed using the computer code, Monte Carlo N-particle eXtended (MCNPX) to calculate photon scattering effects, i.e., to determine scattered, non-resonant background and the notch refilling in transmission measurements. We found that MCNPX underestimated the non-resonant elastic scattering at backwards angles by multiple orders of magnitude mainly due to a flawed treatment of Rayleigh scattering in the code. We corrected this shortcoming and confirmed that the improved treatment of the elastic scattering results in much better agreement with experimental data. Also added to the code was the ability to simulate NRF physics by adding known nuclear resonance data to the photonuclear Evaluated Nuclear Data Files (ENDF), which when processed by using the nuclear data processing code, NJOY, produce data libraries that could be used by MCNPX to fully model NRF measurements.

The main effort in this study was focused on analyzing and evaluating NRF-based measurement

methods using the developed modeling tools. First, we assessed the potential capabilities of 
backscatter and transmission systems that could be built with currently available instrumentation, i.e., systems that use bremsstrahlung photon sources and spectroscopic $\gamma$-ray detectors.

The backscattering method has three main limitations: a low signal-to-background ratio for the small $\mathrm{Pu}$ concentrations in $\mathrm{SNF}$, a high background from the radioactive decay of the fission products in the spent fuel, and a strong dependence on depth of the NRF signal. These difficulties cannot be overcome when using bremsstrahlung sources and available detectors, and thus render this approach not viable for the precise measurement of low concentrations in SNF of ${ }^{239} \mathrm{Pu}$, which is most abundant $\mathrm{Pu}$ isotope in $\mathrm{SNF}$ and is the only isotope whose NRF cross sections have been measured.

The transmission method provides two important advantages: first, detectors can be shielded from $\gamma$ rays emitted from the fission products in the SNF, and second, the measurement sensitivity is not depth dependent. In this method the areal density of the $\mathrm{Pu}$ isotope is derived from the decreased intensity of NRF peaks in the measured spectrum. This decrease is on the order of $0.5 \%$ for the known ${ }^{239} \mathrm{Pu}$ resonances, implying very good counting statistics are needed to determine this quantity with an uncertainty of several percent or better. Due to the high flux of mostly lower-energy, non-resonantly scattered photons associated with the bremsstrahlung beam, count rate limited detectors need to be operated behind thick filters, which reduce the low NRF count rate even further. Sufficiently precise measurements of $\mathrm{Pu}$ isotopic concentrations in SNF would require 10's to 100's of hours, a very intense bremsstrahlung source, and a very large detector array. For example, we estimated that a measurement of $0.4 \%{ }^{239} \mathrm{Pu}$ with a few percent statistical error would take about 24 hours using a detector array that fills a significant fraction of the backwards hemisphere.

Quasi-monoenergetic photon sources such as Laser Compton Scattering (LCS) sources that produce narrow beams of $2-2.5 \mathrm{MeV}$ photons with an energy spread of less than $10 \mathrm{keV}$ could potentially enable sufficiently sensitive NRF measurements to be achieved in a reasonable amount of time. Disregarding source intensity limitations, the time required for a transmission measurement of ${ }^{239} \mathrm{Pu}$ in a SNF assembly could be reduced by more than a factor of 100 , compared to a bremsstrahlung source, when using a photon source with an energy spread of $1 \mathrm{keV}$ and an intensity of $6 \times 10^{8} \mathrm{ph} / \mathrm{eV} / \mathrm{s}$. However, LCS sources currently under development are not this intense and are pulsed at repetition rates below $1 \mathrm{kHz}$. Very high instantaneous beam intensities may necessitate threshold detectors, such as Cherenkov detectors, that can measure an integrated signal. Using such detectors, measurement times would be limited only by the intensity of the photon source.

Interestingly, threshold detectors would also make backscatter measurements with high-intensity, pulsed photon sources possible. Gating the detectors so that they are sensitive only during the short, sub-nanosecond pulses could reduce the background from the radioactivity of the SNF by many orders of magnitude. In a best-case scenario, a measurement with $1 \%$ statistical uncertainty could be accomplished with a $1 \times 10^{8} \mathrm{ph} / \mathrm{eV} / \mathrm{s}$ photon source and an ideal threshold detector in roughly $10 \mathrm{~min}$. While this indicates promise, neither detector nor photon source presently exist.

In all configurations discussed above the isotopic content is measured in the volume that is defined by the intersection of the photon beam with the fuel assembly. Measurement of lower Pu concentrations in a volume could indicate fuel pin removal. By scanning the length and width of the assembly during a measurement the average $\mathrm{Pu}$ content could be determined. It is important to note that ${ }^{240} \mathrm{Pu}$, as an even mass number isotope, likely has significantly larger resonances than ${ }^{239} \mathrm{Pu}$ that could result in ten times higher measurement sensitivities. In combination with other 
data a ${ }^{240} \mathrm{Pu}$ measurement may allow the determination of total $\mathrm{Pu}$, or a ${ }^{239} \mathrm{Pu} /{ }^{240} \mathrm{Pu}$ measurement may be used to determine burn-up.

We conclude from the results of this study that the potential of NRF-based methods for direct, isotope-specific measurements cannot presently be realized and applied to the practical measurement of ${ }^{239} \mathrm{Pu}$ in $\mathrm{SNF}$ assemblies. Achieving the required sensitivity and accuracy will likely require quasi-monoenergetic photon sources with intensities that are two orders of magnitude higher than those currently being designed or proposed. Substantial progress in electron accelerator and laser technologies is still needed before suitable sources with the required capabilities and acceptable size and cost can be constructed. Other needed future work includes the measurement of NRF states for other isotopes, particularly ${ }^{240} \mathrm{Pu}$ and ${ }^{242} \mathrm{Pu}$, development of suitable $\gamma$-ray detector systems, and a more in-depth investigation of potential systematic errors and issues of implementation. 


\section{Contents}

1 Introduction $\quad 1$

2 Quantitative Measurements using NRF 2

2.1 Nuclear Resonance Fluorescence . . . . . . . . . . . . . . . . . . . . . . . . . . . 2

2.1.1 NRF $\gamma$-ray Angular Distributions . . . . . . . . . . . . . . . . . . . 3

2.1.2 Thermal Motion and Nuclear Recoil . . . . . . . . . . . . . . . . . . 4

2.2 Measurement Methods . . . . . . . . . . . . . . . . . . . . . 8

2.2.1 Backscatter Method . . . . . . . . . . . . . . . . . . 8

2.2.2 Transmission Method . . . . . . . . . . . . . . . . . . 9

2.2.3 Measurement Uncertainties . . . . . . . . . . . . . . . . . 11

2.2.4 $\mathrm{Pu}$ Content in Spent Fuel . . . . . . . . . . . . . . . . . . . . . . . . . . . . . . . . . . .

2.3 Instrumentation . . . . . . . . . . . . . . . . . . . . . . . . 12

2.3.1 Photon Sources . . . . . . . . . . . . . . . . . 12

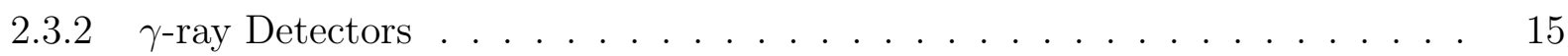

2.3.3 Threshold $\gamma$-ray Detectors . . . . . . . . . . . . . . . . . 17

3 Modeling - Analytical and MCNPX 20

3.1 Backscatter Analytical Model . . . . . . . . . . . . . . . . . . . 20

3.1.1 NRF Count Rates in Backscatter Assay . . . . . . . . . . . . . . . . . . . 20

3.2 Transmission Model . . . . . . . . . . . . . . . . . . . . . . . . 22

3.2.1 Transmission Assay: NRF and Background Signal Rates . . . . . . . . . . 22

3.2.2 Analytical Considerations . . . . . . . . . . . . . . . . . . . 25

3.2.3 Ray-Tracing Algorithm . . . . . . . . . . . . . . . . . . . . 27

3.2.4 Approximate NRF Cross Section Models . . . . . . . . . . . . . . . 29

3.2.5 Relating Attenuation Measurement to $\mathrm{Pu}$ Content in Spent Fuel . . . . . . 31

3.3 MCNPX Modeling . . . . . . . . . . . . . . . . . . . . . . 34

3.3.1 Addition of Available NRF Resonance Data to ENDF and MCNPX Datafiles 35

3.3.2 MCNPX Simulation of Backscatter Measurements with Bremsstrahlung . . 37

3.3.3 MCNPX Simulation of Resonant Attenuation . . . . . . . . . . . . . . 37

3.3.4 Notch Refilling and Transmission Assay Complications . . . . . . . . . . . 38

4 Determining Pu in Spent Fuel Using Available Technology 41

4.1 Backscatter Measurement of Spent Fuel Pins . . . . . . . . . . . . . . . . . . . . . 41

4.1.1 Alternative Backscatter Schemes . . . . . . . . . . . . . . 45

4.2 Transmission NRF Assay . . . . . . . . . . . . . . . . . . . . . . . . 46

4.2.1 Expected Precision for Measurement of Actinides in Spent Fuel using HPGe

Detector Array . . . . . . . . . . . . . . . . . . . . 46

4.2.2 Measurement of $\mathrm{Pu}$ in Spent Fuel using Alternative Detector Types . . . . 47

$4.3 \mathrm{Pu}$ Assay with Bremsstrahlung Sources Conclusions . . . . . . . . . . . . . . . . . 48 
5 Determining $\mathrm{Pu}$ in Spent Fuel Using Quasi-monoenergetic Photon Sources $\mathbf{5 0}$

5.1 Backscatter Measurement Using Quasi-monoenergetic Photons . . . . . . . . . . . 50

5.1 .1 Backscattered Photon Spectra . . . . . . . . . . . . . . . . 50

5.1.2 Influence of Spent Fuel Radioactivity on Backscatter Measurement . . . . 54

5.1 .3 Measurement of Other Rows of Pins . . . . . . . . . . . . . . . 56

5.1 .4 Use of Threshold Detectors . . . . . . . . . . . . . . . . . . 56

5.1.5 Conclusions for Backscatter Measurements Using Quasi-monoenergetic Sources 57

5.2 Transmission Measurements Using Quasi-monoenergetic Sources . . . . . . . . . . 58

5.2.1 Computed Photon Spectra using Quasi-monoenergetic Sources . . . . . . . 60

5.2.2 Source Decomposition Method - Defining Relative Weights . . . . . . . . . 64

5.2.3 Measurement Parameter Estimates Based on Source Energy Decomposition 65

6 Conclusions and Future Work

A Transmission Nuclear Resonance Fluorescence Measurements of ${ }^{238} \mathbf{U}$ in Thick Targets $\quad \mathbf{7 3}$

A.1 Measurement Summary _. . . . . . . . . . . . . . . . . . . . 73

A.2 Introduction and Experimental Goals . . . . . . . . . . . . . . . . 73

A.3 Notch Refill . . . . . . . . . . . . . . . . . . . . . . . . . . . . 73

A.4 Experimental Setup . . . . . . . . . . . . . . . . . . . . . . . 74

A.5 Data Analysis . . . . . . . . . . . . . . . . . . . . . 76

A.6 Areal Density Measurement _. . . . . . . . . . . . . . . . . . . 78

A.7 Conclusion . . . . . . . . . . . . . . . . . . . . 80

B Used Fuel Radioactivity Calculations $\quad 83$

B.1 Unshielded Photon Flux . . . . . . . . . . . . . . . . . . . . . . . 84

B.1.1 Shielded Detector Configuration . . . . . . . . . . . . . . . . 87

B.1.2 Simplifying Assumptions . . . . . . . . . . . . . . . . . . . . . . . . 89

$\begin{array}{ll}\text { C Finite Element Treatment of Target Geometry } & 91\end{array}$

D Rayleigh Scattering in MCNP

D.1 Introduction . . . . . . . . . . . . . . . . . . . . . . . . 93

D.2 Form Factor Sampling Methodology . . . . . . . . . . . . . . . . . . . . 95

D.2.1 Sampling a Simple PDF . . . . . . . . . . . . . . . . . 96

D.2.2 Rejection-Sampling Method . . . . . . . . . . . . . . . . . 96

D.3 Sampling Rayleigh Scattering Cross Sections . . . . . . . . . . . . . . . 98

D.3.1 Integrated Form Factors . . . . . . . . . . . . . . . . . . . . 999

D.4 Description of MCNPX Patch 'Rayleigh Fix' . . . . . . . . . . . . . . . . . . 99

$\begin{array}{lr}\text { E Addition of NRF Data to Photonuclear Datafiles } & 103\end{array}$

E.1 Description of Datafile Creation Method . . . . . . . . . . . . . . . . . 103

E.1.1 NRF Data Search . . . . . . . . . . . . . . . . . . . . . . . . . . 104

E.1.2 NRF $\gamma$-ray Energy Description and RIPL2FIX.EXE . . . . . . . . . . . . 104

E.1.3 NRFXSSI Step 1: Information Gathering . . . . . . . . . . . . . . . 105

E.1.4 NRFXSSI Step 2: DLSL Data Trimming . . . . . . . . . . . . . . . 106

E.1.5 NRFXSSI Step 3: NRF Cross Section Analysis . . . . . . . . . . . . 106

E.1.6 NRFXSSI Step 4: $\gamma$-ray Cascade Analysis . . . . . . . . . . . . . . . 107

E.1.7 NRFXSSI Step 5: Data Formatting and Integration . . . . . . . . . . 109

E.1.8 NRFXSSI Step 6: Data Insertion into Photonuclear ENDF File . . . . . . 109

E.1.9 ACE file Creation using NJOY . . . . . . . . . . . . . . 110 
E.1.10 NJOY Bug Repair . . . . . . . . . . . . . . . . . . . . . . . . 110

E.1.11 NRF data insertion results . . . . . . . . . . . . . . . . . . . 110

$\begin{array}{lr}\text { F Supporting Documentation of MCNPX Simulations } & 113\end{array}$

F.1 Bremsstrahlung Source Simulations . . . . . . . . . . . . . . . . . . . . . . . 113

F.2 Photon Penetration Through Thick Shielding _ . . . . . . . . . . . . . 115

F.2.1 Comparison to Published Build-up Factors . . . . . . . . . . . . . . 117

F.2.2 Shielding Calculations for Reduction of Source Bremsstrahlung Flux . . . . 118

F.3 Backscattered Photon Flux Calculations . . . . . . . . . . . . . . . . . . 121

F.4 Detector Response Modeling . . . . . . . . . . . . . . . . . . . . . . . . . . 124

F.4.1 Model Geometry . . . . . . . . . . . . . . . . . . . . . . . . . . . . . . 124

F.5 Electron Stopping Treatment in MCNP . . . . . . . . . . . . . . . . . . 128

F.6 Thick-Target Bremsstrahlung Model . . . . . . . . . . . . . . . . . . . 128 


\section{List of Acronyms}

$\mathrm{ACE}$

$\mathrm{ADC}$

$\mathrm{CDF}$

CW

DBLP

DLSL

DU

ENDF

ENSDF

EPDL

ETRAN

FEL

FOM

FWHM

GDR

$\mathrm{HI} \gamma \mathrm{S}$

HPGe

HVRL

IAEA

IOI

LBNL

LLNL

LCS

MCNP

MCNPX

NDA

NGSI

NIST

NJOY

NRES

NRFXSSI

NRF

PDF

PWR

QM

RIPL

SNF

TD

TTB

wt. $\%$

$\mathrm{XCOM}$ nuclear data file format used by MCNP

Analogue-to-Digital Converter

Cumulative probability Distribution Function

Continuous Wave - particle accelerators that are not pulsed

Doppler-broadened Lorentzian cross section Profile

Discrete Level Scheme Library

Depleted Uranium

Evaluated Nuclear Data File

Evaluated Nuclear Structure Data File

Evaluated Photon Data Library (97)

computer program Electron TRANsport

Free Electron Laser

Figure Of Merit

Full-Width at Half-Maximum

Giant Dipole Resonance

High Intensity $\gamma$-ray Source

High-Purity Germanium detector

High Voltage Research Laboratory

International Atomic Energy Agency

Isotope of Interest for transmission assay measurements

Lawrence Berkeley National Laboratory

Lawrence Livermore National Laboratory

Laser Compton Scattering photon sources

computer program Monte Carlo N-Particle

computer program Monte Carlo N-Particle eXtended

Non-Destructive Assay

Next Generation Safeguards Initiative

National Institute of Standards and Technology

daughter nuclear data processing code of program MINX:

Multi-group Interpolation of Nuclear X-Sections

Non-Resonant Elastic Scatter

Computer code, NRF cross(X) SectionS Inserter

Nuclear Resonance Fluorescence

Probability Distribution Function

Pressurized Water Reactor

Quasi-Monoenergetic photon source

Reaction/Interaction Parameter Library

Spent Nuclear Fuel

Transmission detector (comprised of IOI)

Thick-Target Bremsstrahlung model

weight percent

NIST X-ray attenuation coefficient database 


\section{Introduction}

The Next Generation Safeguard Initiative (NGSI) of the U.S Department of Energy is supporting a multi-lab/university collaboration to quantify the plutonium $(\mathrm{Pu})$ mass in spent nuclear fuel (SNF) assemblies and to detect the diversion of pins with non-destructive assay (NDA) methods. The following 14 NDA techniques are being studied: Delayed Neutrons, Differential Die-Away, Differential Die-Away Self-Interrogation, Lead Slowing Down Spectrometer, Neutron Multiplicity, Passive Neutron Albedo Reactivity, Total Neutron (Gross Neutron), X-Ray Fluorescence, ${ }^{252}$ Cf Interrogation with Prompt Neutron Detection, Delayed Gamma, Nuclear Resonance Fluorescence, Passive Prompt Gamma, Self-integration Neutron Resonance Densitometry, and Neutron Resonance Transmission Analysis[1, 2]. Understanding and maturity of the techniques vary greatly, ranging from decades old, well-understood methods to new approaches. Nuclear Resonance Fluorescence (NRF) is a technique that had not previously been studied for SNF assay or similar applications. Since NRF generates isotope-specific signals, the promise and appeal of the technique lies in its potential to directly measure the amount of a specific isotope in an SNF assay target $[3,4]$.

The objectives of this study were to design and model suitable NRF measurement methods, to quantify capabilities and corresponding instrumentation requirements, and to evaluate prospects and the potential of NRF for SNF assay. The main challenge of the technique is to achieve the sensitivity and precision, i.e., to accumulate sufficient counting statistics, required for quantifying the mass of $\mathrm{Pu}$ isotopes in SNF assemblies. Systematic errors, considered a lesser problem for a direct measurement and only briefly discussed in this report, need to be evaluated for specific instrument designs in the future. Also, since the technical capability of using NRF to measure Pu in SNF has not been established, this report does not directly address issues such as cost, size, development time, nor concerns related to the use of $\mathrm{Pu}$ in measurement systems.

This report discusses basic NRF measurement concepts, i.e., backscatter and transmission methods, and photon source and $\gamma$-ray detector options in Section 2. An analytical model for calculating NRF signal strengths is presented in Section 3 together with enhancements to the MCNPX code[5] and descriptions of modeling techniques that were drawn upon in the following sections.

Making extensive use of the model and MCNPX simulations, the capabilities of the backscatter and transmission methods based on bremsstrahlung or quasi-monoenergetic photon sources were analyzed as described in Sections 4 and 5. A recent transmission experiment is reported on in Appendix A. While this experiment was not directly part of this project, its results provide an important reference point for our analytical estimates and MCNPX simulations. Used fuel radioactivity calculations, the enhancements to the MCNPX code, and details of the MCNPX simulations are documented in the other appendices. 


\section{Quantitative Measurements using NRF}

Nuclear resonance fluorescence is the process by which a nucleus absorbs energy in the form of electromagnetic radiation, is excited to a nuclear state, and then subsequently de-excites to the ground state by the emission of one or more $\gamma$ rays. As will be discussed in detail later, the angular distribution of re-emitted photons is governed by the angular momenta of the states involved in the process, and their energy is slightly below that of the NRF-inducing photons due to the nuclear recoil.

The energies of the photon that induced the initial excitation, and the energy of the re-emitted photon(s) are characteristic of the specific state that underwent NRF, and, therefore, characteristic of the isotope. Photons produced during de-excitation of a NRF state are referred to herein as NRF $\gamma$ rays. Because NRF states are simply excited nuclear states, possible NRF $\gamma$-ray energies range from tens of $\mathrm{keV}$ up to many $\mathrm{MeV}$. However, for the purpose of using NRF to assay materials, photons of energy between 1.5 and $4 \mathrm{MeV}$ are most useful.

Both the elastic and inelastic scattering of photons may contribute to the background. However, physical properties of these scattering mechanisms cause the background intensity for the detection of NRF $\gamma$ rays to be a function of both the interrogating beam's energy spectrum and the geometry of the detection setup. Measurements of NRF $\gamma$ rays at backwards angles, relative to the interrogating beam direction, yields backgrounds that are significantly reduced compared to the forward angles.

\subsection{Nuclear Resonance Fluorescence}

In the context of assaying materials, NRF is usually induced by exciting nuclear states with a beam of photons. The subsequent NRF $\gamma$ rays are measured using photon detectors such as high-purity germanium (HPGe) or scintillation detectors. Because NRF states correspond to excited nuclear levels, the photo-absorption and NRF $\gamma$-ray energies identify the nucleus that has undergone NRF. The identification is analogous to passive $\gamma$-ray spectroscopy used to identify decaying radioactive nuclides.

If assay geometry and NRF cross section are known, measuring the rate at which NRF occurs allows the number of atoms of the corresponding isotope to be determined. The rate at which a nuclide undergoes NRF in thin targets is given by

$$
R=\int N \Phi(E) \sigma(E) d E
$$

Thicker targets require geometrical corrections due to the fact that the energy-dependent photon flux, $\Phi(E)$ changes as it traverses the target. This will be discussed in Section 3.1.1.

The cross section for photo-excitation of a nuclear state is given by the Breit-Wigner distribution:

$$
\sigma(E)=\pi g \frac{(\hbar c)^{2}}{E^{2}} \frac{\Gamma \Gamma_{0}}{\left(E-E_{c}\right)^{2}+(\Gamma / 2)^{2}}
$$


where $\Gamma$ is the full-width at half maximum (FWHM) of the state and is related to the state's mean lifetime, $\tau$, by

$$
\Gamma=\frac{\hbar}{\tau}
$$

$\Gamma_{0}$ is the partial width of the state for decay by $\gamma$-ray emission to the ground state, $E_{c}$ is the centroid energy of the resonance, and $g$ is a statistical factor equal to the ratio of the number of spin states available for the excitation to the number of initial spin states. For NRF events where the initial nuclear state is the ground state it is

$$
g=\frac{2 J+1}{2\left(2 J_{0}+1\right)}
$$

where $J$ and $J_{0}$ are the angular momentum quantum numbers of the excited and ground states, respectively. There are $2 J+1$ magnetic substates for a state of angular momentum $J$, and the additional factor of 2 in the denominator is due to the fact that the photons inducing excitation can have two possible helicities[4].

The probability for de-excitation of a state by a specific mode (i.e., neutron emission, $\gamma$-ray emission, etc.) yielding a lower-energy state can be defined by the state's partial width for that mode,

$$
p_{i}=\frac{\Gamma_{i}}{\sum_{j} \Gamma_{j}}=\frac{\Gamma_{i}}{\Gamma}
$$

where we have used

$$
\Gamma=\sum_{j} \Gamma_{j}
$$

implying that the summation over $j$ includes all possible de-excitation modes. Combining equations 2.2 and 2.5, we note that the cross section for NRF to occur via emission of a single $\gamma$ and transition to the ground state, is given by

$$
\sigma_{\mathrm{GS}}(E)=\frac{\Gamma_{0}}{\Gamma} \sigma(E)=\pi g \frac{(\hbar c)^{2}}{E^{2}} \frac{\Gamma_{0}^{2}}{\left(E-E_{c}\right)^{2}+(\Gamma / 2)^{2}}
$$

In the rest-frame of the nucleus, photon wave functions within its vicinity become successively smaller with increasing angular momentum. Because of this, the probability for interaction with the nucleus similarly decreases with increasing angular momentum, and photonuclear interactions will preferentially occur via the lowest angular momentum transfer possible[6].

\subsubsection{NRF $\gamma$-ray Angular Distributions}

NRF is generally considered to only occur between states that differ by 2 or fewer units of angular momentum. The angular distribution of NRF $\gamma$ rays, relative to the NRF-inducing radiation can be described by the same spin algebra that is used to define angular correlations in $\gamma$-ray cascades[7].

The cross section for NRF $\gamma$ ray emission at a direction, $\theta$, relative to the incident photon beam is given by

$$
\sigma(\theta)=W(\theta) \sigma
$$


where, $W(\theta)$, denotes the angular correlation function. It is a normalized function, i.e.,

$$
\int_{0}^{2 \pi} d \phi \int_{0}^{\pi} \sin (\theta) d \theta W(\theta)=4 \pi
$$

and (un-normalized) values for $W(\theta)$ were derived for absorption of un-polarized dipole or quadrupole radiation followed by emission of dipole or quadrupole radiation in reference[7]. For a more in depth discussion of angular dependence see reference[4].

${ }^{239} \mathrm{Pu}$ has a spin-1/2 ground state and only transitions that return to the ground state are considered in this discussion. Transitions of the form $1 / 2 \rightarrow 1 / 2 \rightarrow 1 / 2,1 / 2 \rightarrow 3 / 2 \rightarrow 1 / 2$, and $1 / 2 \rightarrow 5 / 2 \rightarrow 1 / 2$, are all expected to be possible in $\operatorname{NRF}(\Delta J \leq 2)$. Furthermore, the multipolarity of the $1 / 2 \rightarrow 3 / 2$ excitation may be either dipole or quadrupole (due to vector spin addition rules), and likewise for the $3 / 2 \rightarrow 1 / 2$ de-excitation. The angular distributions predicted for purely dipole-dipole and purely quadrupole-quadrupole transitions happen to be the same, but if the excitation occurs via quadrupole and the de-excitation via dipole (or vice versa) the expected $W(\theta)$ differs significantly. Because the actual intensities of quadrupole versus dipole transitions (commonly referred to as the mixing ratio, $\delta$ ) can vary from state to state, any possible $W(\theta)$ distribution combination is allowed.

The cases of ground-state to ground-state NRF on spin $1 / 2$ nuclei, such as ${ }^{239} \mathrm{Pu}$ is presented in Figure 2.1. The red curve indicates a $1 / 2 \rightarrow 3 / 2 \rightarrow 1 / 2$ transition where both the photon multipolarities were both either dipole or quadrupole, whereas the green curve indicates a transition where one photon was dipole and the other quadrupole. The area between the curves has been shaded gray to indicate that any possible angular correlation function between these two extremes is physically possible. Finally, the black curve indicates the sequence $1 / 2 \rightarrow 5 / 2 \rightarrow 1 / 2$ (assuming octupole radiation is negligible) is only described by quadrupole transitions. As long as the known excited ${ }^{239} \mathrm{Pu} \mathrm{NRF}$ are not spin $5 / 2$, there does not appear to be a preferred measurement angle for ${ }^{239} \mathrm{Pu}$. If there is a spin 5/2 state, the measurement at angles larger than $230^{\circ}$ would be preferable; otherwise near to $90^{\circ}$ would also be more favorable than a measurement near $213^{\circ}$, which could reduce count rates by up to $15 \%$.

Measurement of an even mass number isotope of $\mathrm{Pu}$ would most probably result in the $0 \rightarrow$ $1 \rightarrow 0$ spin sequence, which favors measurement at angles larger than $215^{\circ}$ and disfavors $90^{\circ}$ measurements by up to $25 \%$. Conversely, measurement of a $0 \rightarrow 2 \rightarrow 0$ spin sequence favors large angles or $90^{\circ}$ measurements and strongly disfavors measurements at angles near $218^{\circ}$.

\subsubsection{Thermal Motion and Nuclear Recoil}

The NRF process is connected to the environment of the nucleus due to the fact that nuclei, as part of atoms, are not stationary, but are always in thermal motion. In the case where NRF is induced on a nucleus comprising a gaseous atom or part of a gaseous molecule, the velocity distribution of the nucleus is defined by a Maxwell-Boltzmann probability distribution

$$
P(v)=\sqrt{\frac{M}{2 \pi k_{B} T}} \exp \left(-M v^{2} / 2 k_{B} T\right)
$$

where $M$ is the mass of the atom or molecule, $k_{B}$ is Boltzmann's constant, $\left(1.3806503 \times 10^{-23} \mathrm{~m}^{2} \mathrm{~kg} \mathrm{~s}^{-2} \mathrm{~K}^{-1}\right)$, and $T$ is the absolute temperature of the gas. 


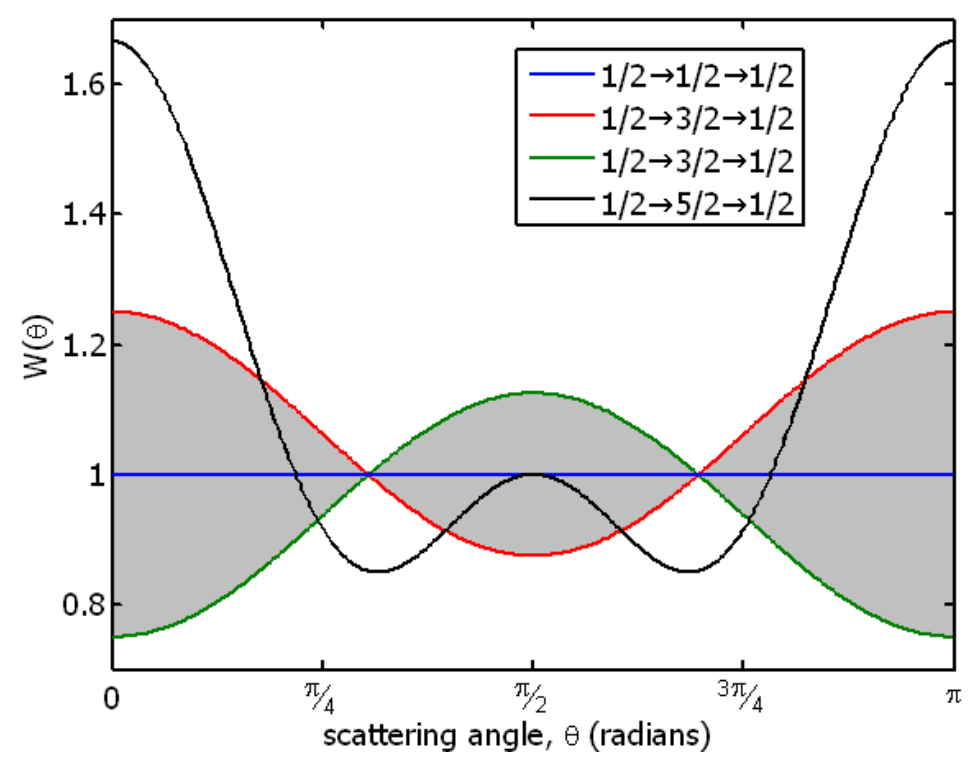

Figure 2.1 The angular correlation functions, $W(\theta)$ for NRF between states of initial and final spin $1 / 2$ allowed by dipole and quadrupole radiation. The red curve indicates a $1 / 2 \rightarrow 3 / 2 \rightarrow 1 / 2$ transition where both the photon multipolarities were both either dipole or quadrupole, whereas the green curve indicates a transition where one photon was dipole, and the other quadrupole. The area between the curves has been shaded gray to indicate that any possible angular correlation function between these two extremes is physically possible.

For a photon of energy $E$, a nucleus moving at velocity $v$ will experience a photon of shifted energy, $E^{\prime}$ due to the Doppler effect

$$
E^{\prime}=E\left(\frac{1+v / c}{\sqrt{1-(v / c)^{2}}}\right) \approx E\left(1+\frac{v}{c}\right)
$$

where we have assumed that the velocity of the nucleus is very small compared to the speed of light.

Substituting, we find that the effective energy distribution is given by

$$
P\left(E^{\prime}\right) d E^{\prime}=\frac{1}{\Delta \sqrt{2 \pi}} \exp \left(-\left(E^{\prime}-E\right)^{2} / 2 \Delta^{2}\right) d E^{\prime}
$$

where we have used $d E^{\prime} \approx \frac{E^{\prime}}{c} d v$ and

$$
\Delta=E \sqrt{\frac{k_{B} T}{M c^{2}}}
$$

is the standard deviation of the distribution. We commonly refer to the Doppler width of a broadened resonance as

$$
\Gamma_{D} \equiv 2 \sqrt{2 \ln 2} \Delta=2.3548 \Delta
$$

because it describes the FWHM of the distribution in equation $2.12^{1}$.

\footnotetext{
${ }^{1}$ It should be noted that another common definition of the Doppler width is $\sqrt{2} \Delta$. This definition is not used in this document.
} 
The effective photo-absorption cross section for a photon incident upon a collection of nuclei with a velocity distribution given by equation 2.10 is then found by averaging the Doppler-shifted energy probability distribution with the absorption cross section[3]:

$$
\sigma_{D}(E)=\int_{0}^{\infty} d E^{\prime} \sigma\left(E^{\prime}\right) P\left(E^{\prime}\right)
$$

Substituting equations 2.2 and 2.12, we have the Doppler-broadened Lorentzian profile (DBLP):

$$
\sigma_{D}(E)=\frac{\sqrt{\pi} g(\hbar c)^{2} \Gamma \Gamma_{0}}{\sqrt{2} \Delta} \int_{0}^{\infty} d E^{\prime} \frac{1}{\left(E^{\prime}-E_{c}\right)^{2}+(\Gamma / 2)^{2}} \frac{\exp \left(-\left(E^{\prime}-E\right)^{2} / 2 \Delta^{2}\right)}{E^{\prime 2}}
$$

As an illustrative example, Figure 2.2 demonstrates the effect that a Maxwell-Boltzmann energy distribution can have on the effective resonance shape that an incident photon experiences. This case describes a $2.175 \mathrm{MeV}$ centroid energy $\left(E_{c}\right){ }^{238} \mathrm{U}$ resonance with a width, $\Gamma=54 \mathrm{meV}$ and that de-excites by photon emission to the ground state $68 \%$ of the time $\left(\Gamma_{0} / \Gamma=0.68\right)$. The spin of the ground state is 0 , and that of the excited state is assumed to be 1 , such that $g=3 / 2$. This distribution is also broadened due to the motion of the atoms, which have been described by a Maxwell-Boltzmann velocity distribution for ${ }^{238} \mathrm{U}$ at $300^{\circ} \mathrm{K}: \Gamma_{D}=1.78 \mathrm{eV}$. The corresponding energy probability distribution was multiplied by $\int \sigma(E) d E$, and is shown as the green line in Figure 2.2. Equation 2.16 was numerically integrated for these parameters for an array of incident photon energies, and the resulting effective cross sections are shown as the dots on the red line in Figure 2.2. This example demonstrates that the width of the effective cross section distribution will be broader than that of the energy distribution and that of the natural cross section.

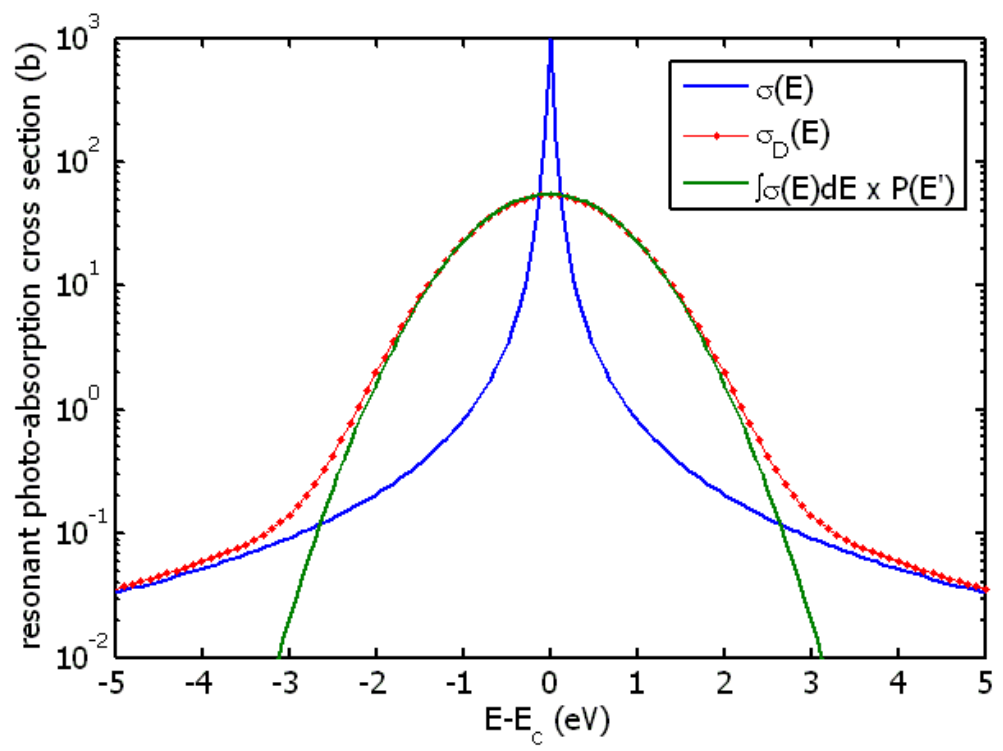

Figure 2.2 Effective resonance shape experienced by an incident photon, for the $2175 \mathrm{keV}$ resonance of ${ }^{238} \mathrm{U}$ at $300^{\circ} \mathrm{K}$. For this resonance, $\Gamma=54 \mathrm{meV}, \Gamma_{0}=37 \mathrm{meV}$, and $g=3 / 2$.

Non-amorphous solids are composed of crystalline arrangements of constituent atoms. These atoms vibrate with thermal energy, which can be described as a phonon. The effects of phonons are most evident when the nuclear recoil energy is not significantly greater than typical phonon 
energies. However, above $\sim 1.5 \mathrm{MeV}$, the influence of crystalline phonons on the energy distribution of the nuclei continues to have measurable effects. In particular, the binding energy of the crystal and the phonon-induced motion causes crystalline atoms to vibrate faster than gaseous atoms at the same temperature. In the limit that $\Delta / \sqrt{2}+\Gamma>>2 k_{B} \theta$, the effective temperature at which equation 2.16 should be evaluated $[3,8]$ is

$$
\frac{T_{e f f}}{T}=3\left(\frac{T}{\theta}\right)^{3} \int_{0}^{\theta / T} t^{3}\left(\frac{1}{\exp (t)-1}+\frac{1}{2}\right) d t
$$

where, $\theta$ is the Debye temperature of the crystal, which is a physical property of a material that is related to the theoretical maximum energy a single phonon can carry in the material.

We further investigate the loose binding limit, $\Delta+\Gamma>>2 k_{B} \theta$ by using equation 2.13 and assuming that $\Gamma$ is smaller than $\Delta$ to re-write this limit. It becomes

$$
\frac{E}{\theta} \sqrt{\frac{T}{A}}>>285[\mathrm{eV} / \sqrt{\mathrm{K}}]
$$

where $A$ is the mass number of the atom in question. This indicates that for the loose binding approximation to be valid at $T=300^{\circ} \mathrm{K}, E$ must be significantly greater than $52 \mathrm{keV}$ for a uranium matrix or $46 \mathrm{keV}$ for a $\mathrm{UO}_{2}$ matrix. All NRF resonances considered herein fall within this limit.

Finally, we consider the influence of momentum conservation on NRF physics. Conservation of momentum requires that when a nucleus of mass, $M$ absorbs a $\gamma$ ray of energy $E_{\gamma}$ and momentum $E_{\gamma} / c$, it will recoil with the $\gamma$ ray's momentum, which translates to an energy

$$
E_{\text {recoil }}=\frac{E_{\gamma}^{2}}{2 M c^{2}}
$$

For example, the $2431 \mathrm{keV}$ resonance of ${ }^{239} \mathrm{Pu}$ corresponds to a recoil energy of $13.2 \mathrm{eV}$. Of particular importance is that the nuclear recoil energy is significantly larger than the Dopplerbroadened width of the resonance.

We can equate the expressions for recoil energy and Doppler widths (assuming $\Gamma_{D}>\Gamma$ ). With the simplifying assumption that $M \approx A m_{n}$, one finds the approximate result that recoil energies equal Doppler widths when

$$
E_{\text {recoil }}=11.4 \mathrm{keV} \times \sqrt{A}
$$

Assuming that the Doppler width of a resonance is larger than the natural line width, and that the centroid energy is sufficient for the loose binding limit to apply, then the NRF recoil energy is larger than the width of a resonance and re-emitted NRF $\gamma$ rays can be assumed to be no longer resonant.

The conservation of momentum also implies that upon resonance absorption, a nucleus will recoil with energy given by equation 2.19. NRF state lifetimes tend to be on the order of $10^{-17}$ to $10^{-12} \mathrm{~s}$, implying that the nucleus will re-emit an NRF $\gamma$ ray before slowing down to thermal velocities. In the event that the $\gamma$ ray is emitted in precisely the same direction as the incident exciting photon, the nucleus would recoil back to its initial velocity. If the $\gamma$ ray is emitted in a different direction, the nucleus will again recoil from $\gamma$-ray emission and the resulting NRF $\gamma$ ray will be lower in energy than the initial photon. Most probably this energy difference will be larger than the Doppler-broadened width of the NRF resonance, and thus the emitted NRF $\gamma$ ray will no longer be resonant. 


\subsection{Measurement Methods}

In this section the development and use of two methods to measure nuclear resonance fluorescence rates for non-destructive materials assay studies are discussed. These methods assume that NRF is detected by a single radiation detector, and that use of multiple detectors would proportionally increase the count rates. The two assay methods considered here are termed backscatter and transmission assay. They differ in how photons undergoing NRF in the assay target are observed. Both methods use a photon source to induce NRF in the irradiated volume of the target material. In backscatter assay, a radiation detector is positioned at backwards angles relative to the incident photon beam trajectory. Transmission assay uses a detection system down-stream of the assay target to measure the excess attenuation of resonant-energy photons in the target. Both methods have advantages and disadvantages that will be discussed in the following sections.

\subsubsection{Backscatter Method}

A schematic drawing of a backscatter NRF assay geometry is shown in Figure 2.3. A source of energetic photons illuminates the target material and one or more radiation detectors measure the photon flux backscattered from the target. The interrogating photons stimulate resonances in the ${ }^{239} \mathrm{Pu}$ within the spent nuclear fuel. These resonances promptly de-excite by nearly isotropic emission of one or more photons. These NRF $\gamma$ rays are mixed in with the photon flux backscattered from the target.

The background, non-resonant photon flux in the backscattered direction relative to the beam incident upon the target is composed of elastic and non-elastically scattered interrogation photons and photons due to radioactive decays in the target. The detectors used are positioned at backwards angles to the target where the background photon flux is lowest and the signal-tobackground ratio the highest. The detectors are shielded from the interrogation source using large amounts of shielding, such as lead or tungsten. To control the count rate seen by the detector due to low energy scattered photons, $511 \mathrm{keV}$ photons, and the radioactivity of the target, relatively thin lead shielding is place in front of the detectors. This shielding is often referred to as a filter. As discussed further in Section 2.3.2 detector energy resolution increases the statistical value of registered NRF counts. Because of this, HPGe detectors are most commonly used for NRF experiments.

The $\gamma$-ray detectors are often collimated to only view a portion of the measured target. For fuel assemblies, this may be a portion of a single fuel pin. Multiple detectors could, in principle, be positioned to view adjacent regions, thereby the entire irradiated portion of the target may be measured simultaneously. Scanning the fuel assembly by slowly shifting its position relative to the beam may yield a complete measurement of the fuel assembly volume.

Figure 2.3 illustrates several design features of a backscatter NRF assay system. First, the radiation detectors are generally positioned at a scattering angle of $\geq 90^{\circ}$ to take advantage of the decreasing intensity of non-resonantly backscattered radiation. Second, the interrogating photon beam is shown to be collimated toward the spent fuel assembly. A third feature is the shielding directly between the detector and the target, labeled as "filter." Filters may consist of up to $\sim 200 \mathrm{~g} / \mathrm{cm}^{2}$ of high- $Z$ material, depending on photon beam characteristics and detector count rate limitations. They are application-specific and will be discussed in specific assay examples in Sections 3.2, 4.1, and 4.2. 


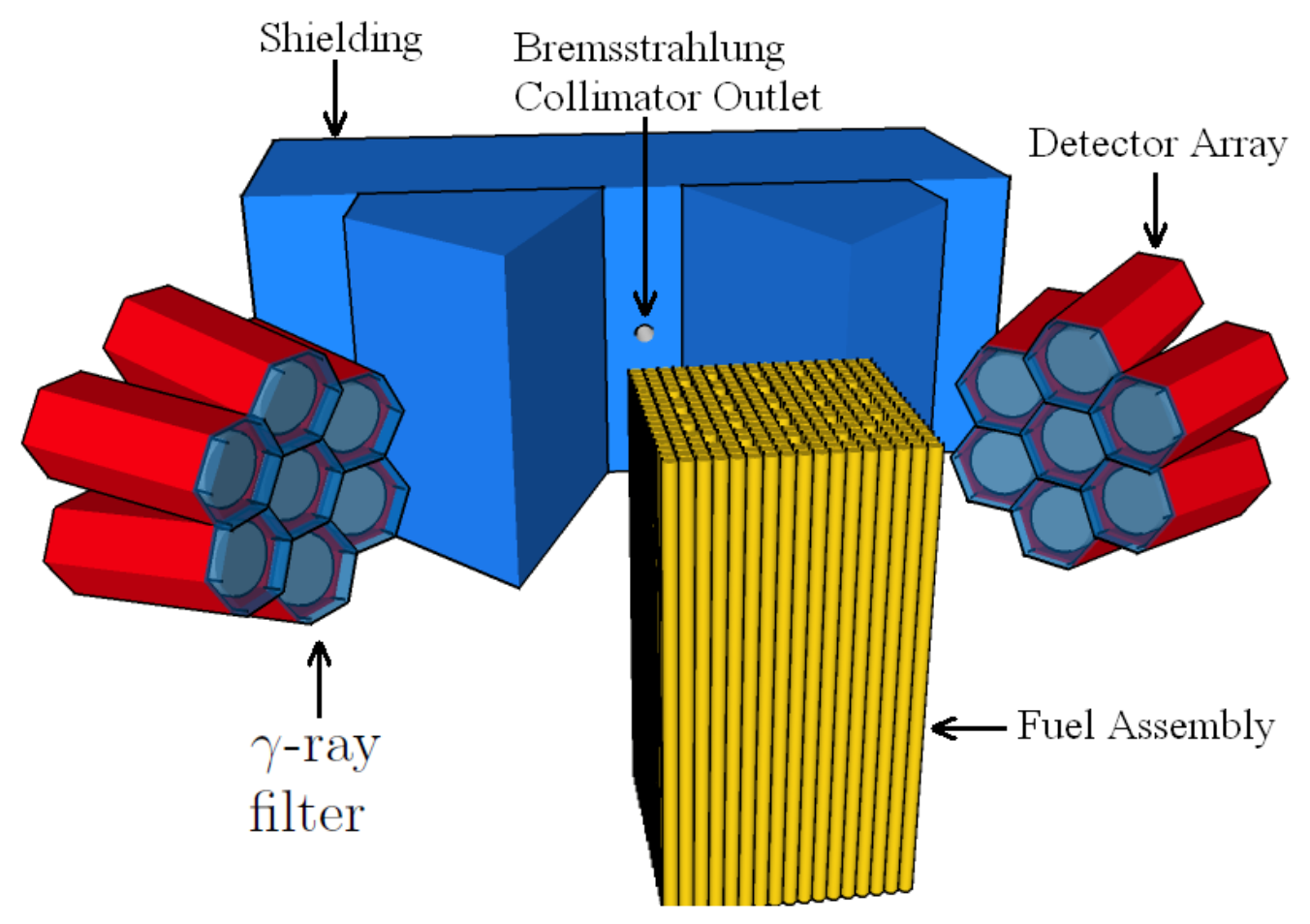

Figure 2.3 Schematic drawing of a bremsstrahlung-induced backscatter NRF assay setup.

\subsubsection{Transmission Method}

The measurement of NRF $\gamma$ rays at backward angles suffers from the disadvantage that radioactivity emitted by the assay target can necessitate thick filters in front of the detector, which subsequently reduces the NRF $\gamma$-ray detection rate. The method is also more sensitive to radiation backscattered from the front of the target rather than from the target as a whole, resulting in potentially biased results for non-homogeneous targets. The transmission method mitigates these disadvantages at the expense of a more complicated assay system and the need for even stronger interrogating photon beams.

The concept of an NRF assay using the transmitted spectrum emanating from the assay target is as follows: an interrogating beam impinges upon the assay target and the spectrum transmitted through the target is then incident upon a thin sheet made of the isotope to be measured called here the transmission detection sheet (TD). The TD, also often referred to as a "witness foil", is ideally a pure $\mathrm{Pu}$ isotope that is to be assayed in the spent fuel assembly. This isotope may be referred to as the isotope of interest (IOI). The TD must be sufficiently thick so that a large fraction of photons at resonant energy stimulate fluorescence; depending on the isotope and its resonances' cross sections this means thicknesses in the $1-10 \mathrm{~mm}$ range. The TD sheet should be large enough to intercept most of the transmitted photon beam. Bremsstrahlung beams could be from a few $\mathrm{cm}$ to the width of the assembly in size, whereas QM photon beams (see Section 2.3.1) may be a few $\mathrm{mm}$ in diameter. Resonant-energy photons that impinge upon the TD may induce NRF. The NRF $\gamma$-rays emanating from the TD are then detected by radiation detectors located at backward angles, relative to the incident bremsstrahlung beam. A schematic diagram of this assay type is shown in Figure 2.4. 


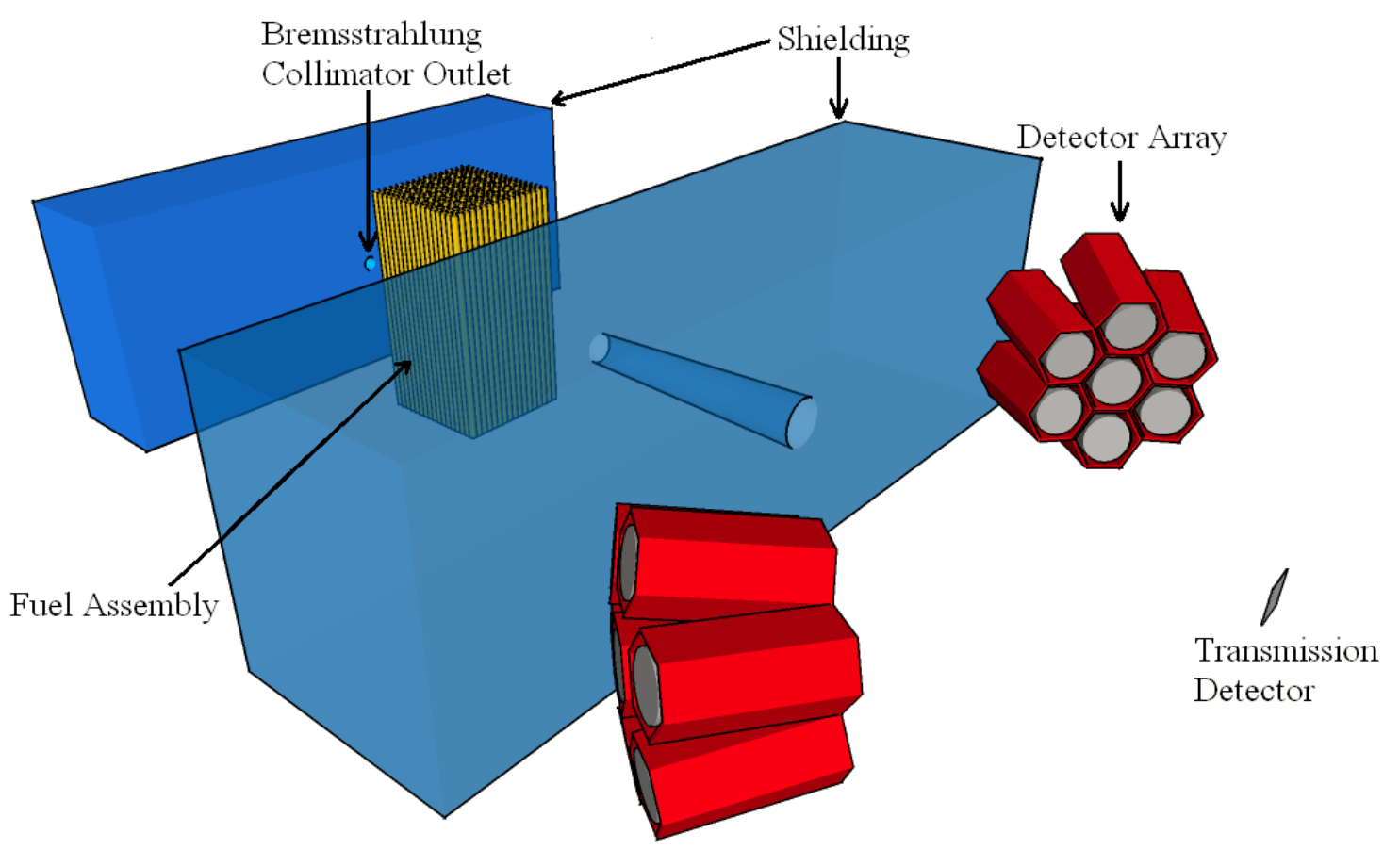

Figure 2.4 Schematic description of a geometry used for a transmission assay measurement.

The sensitive volume of the assay target is defined as the region through which interrogating photons can penetrate and subsequently reach the TD without scatter. The reduced rate at which the TD undergoes NRF is proportional to the intensity of preferential attenuation undergone by resonant-energy photons while traversing the sensitive volume of the target. This intensity is directly correlated to the areal density of the IOI within the sensitive volume of the target. Slow translation of the assay target with respect to the beam and TD during interrogation can produce an averaged measurement of the entire target volume.

For an ideal transmission measurement, the target thickness should be such that the IOI preferentially attenuates a significant fraction of the penetrating resonant-energy photons. Likewise, the source of interrogating photons should be sufficiently intense such that the detectors used to view the TD operate near their rate limit.

For spent fuel, the areal densities of $\mathrm{Pu}$ isotopes are generally very low compared to this ideal case, and the excess attenuation of resonant-energy photons is small. Because of this, and the fact that the radiation detectors are not positioned to directly view the photon flux emitted from the SNF, spent fuel assemblies rather than fuel pins are considered for transmission measurements. So long as the photon source is of sufficient intensity, this is the best case for transmission measurements.

The term notch refill is used to describe the process by which photons incident upon the assay geometry down-scatter to the energy of a resonance and subsequently interact in the transmission detection sheet. The process results in less observed resonant attenuation than would be predicted by consideration of simple exponential attenuation, and therefore neglect of the notch refill phenomenon could result in NRF transmission measurements that systematically under-predict the areal density of the measured isotope in the target.

Photon interaction processes that can induce notch refill include incoherent scatter and brems- 
strahlung emission from photoelectrons. The rate at which notch refill occurs is dependent upon the compositions of the assay target and transmission detection sheet, their respective positioning, the energy and strength of a resonance, and the photon spectrum used for the measurement. Notch refill must be taken into account and corrected for in order to accurately extract ${ }^{239} \mathrm{Pu}$ content from measured spectra. For a more in depth discussion on notch refill see Section 3.3.4.

\subsubsection{Measurement Uncertainties}

A core question this research attempts to answer is what measurement sensitivity, i.e., precision and accuracy, for the assay of ${ }^{239} \mathrm{Pu}$ content in SNF can potentially be achieved. In the context of the NGSI spent fuel research effort we assume that for a technique such as NRF to improve on currently available methods the uncertainty of a direct ${ }^{239} \mathrm{Pu}$ assay should be better than a few percent. The many contributing factors that were considered include interrogating photon source, interrogating photon flux, measurement technique (backscatter vs. transmission), detector filter thickness, detector energy resolution, detector count rate, transmission target thickness, backscatter angle of the detectors, and measurement time. The challenge for a precise measurement of a small concentration of $\mathrm{Pu}$ isotopes in SNF comes from accruing the necessary counting statistics because the integrated resonance cross sections are relatively small. Using the backscatter detection method, the NRF peaks are difficult to precisely measure on the much larger relative background. In the transmission technique only a small change in the NRF peak intensity is observed. In order to achieve practical measurement times stronger resonances or a new generation of high-intensity photon sources and efficient detection systems, as discussed in the following sections of the report, are needed.

The ability to detect removal of pins from used fuel assemblies has also been identified as an important component to the NGSI spent fuel research effort. Because NRF measurement techniques produce signals that are directly related to the quantity of a $\mathrm{Pu}$ isotope present in the irradiated volume, any diversion scenario can, in principle, be detected so long as the statistical uncertainty with which the $\mathrm{Pu}$ isotope is measured is sufficiently small to be sensitive to such a diversion. Given that spent fuel assemblies are generally 8 to 17 pins deep ${ }^{2}$, diversion of a single fuel pin would result in approximately $12.5 \%$ to $5.9 \%$ reduction of $\mathrm{Pu}$ in a row of fuel pin. A NRF measurement system using a narrow beam that could achieve a few percent statistics in ${ }^{239} \mathrm{Pu}$ or ${ }^{240} \mathrm{Pu}$ composition would therefore be capable of detecting diversion of even a single fuel pin.

The systematic errors that may be associated with NRF measurements include the following: Errors in NRF cross sections result in proportionally inaccurate measurements. As discussed later, notch refill may reduce the intensity of measured resonant absorption in a transmission measurement, relative to the predicted by simple analytical models, and will result in systematic errors without precise models that account for notch refill. Overlap of other $\gamma$ ray peaks (other NRF lines or background) can significantly reduce the precision with which the intensity of a resonance may be measured. The photon flux that induces NRF must be precisely known. This may be accomplished by system calibration as long as the beam remains stable. If this method is used, beam instability becomes an important contributor to systematic error. If the flux is determined by an alternative normalizations scheme, such as the $511 \mathrm{keV}$ normalization routine, or by making relative measurements to other NRF lines, beam stability may be less important, however such routines may result in separate systematic errors. Similarly, the uncertainty to which the $\gamma$-ray

\footnotetext{
${ }^{2}$ And neglecting spatial variations of $\mathrm{Pu}$ isotope concentrations within fuel
} 
detectors are calibrated will proportionally induce errors for absolute measurements, but relative measurements made on well-calibrated systems will be less sensitive to absolute knowledge of the $\gamma$-ray detection efficiency. Similar to beam stability, detector stability becomes very important for calibrated systems. Radiation damage to the fuel pins may lead to deformations and may, thus, result in geometric differences between measurements comparing irradiated assemblies to a reference assembly. Although these are likely to be small non-negligible systematic errors may result. Likewise, the uncertainty to which non-resonant attenuation coefficients are known will adversely effect the precision at which geometric effects may be corrected for in relative and absolute measurement schemes.

Model calculation corrections can be applied to correct for notch refill and possibly peak overlap. Improved measurements of NRF cross sections using newer and more intense bremsstrahlung sources are necessary. Other systematic errors can be addressed and minimized through calibration, which is expected to be necessary for any measurement system.

The analyses presented in this report assume that the NRF measurement is conducted on a fuel assembly that has been removed from a cooling or storage pond. This need not necessarily be the case. The presence of water has little effect on any portion of NRF measurements except the additional attenuation of beam photons. Using the minimum depth of $25 \mathrm{~cm}$ of water through which one can conceive of making a NRF measurement, would reduce the intensity of resonant-energy photons by a factor of $\sim 1 / 3$. Therefore for a given measurement geometry and beam intensity, the sensitivity of a measurement made in water would be reduced by a factor of

approximately $1 / \sqrt{3}$. Water can also increase the notch refill phenomenon, as will be discussed in Section 3.3.4.

\subsubsection{Pu Content in Spent Fuel}

In reference[9], Phillips presents calculations of the concentrations of $\mathrm{Pu}$ isotopes in spent fuel as a function of fuel burn-up. These values were calculated using the computer code CINDER[10] and are shown in Figure 2.5. Phillips indicates that $\mathrm{Pu}$ concentrations are expected to be accurate to $\pm 10 \%$ when the reactor spectrum is well-known, and up to $\pm 25 \%$, if only the type of reactor is known. Because the $\mathrm{Pu}$ concentrations are a function of the reactor type, the $\mathrm{Pu}$ concentration values are used to estimate a region of relevant target areal densities. For a typical $15 \times 15$ fuel assembly, the average areal densities of ${ }^{239} \mathrm{Pu}$ and ${ }^{240} \mathrm{Pu}$ traversed by photons normally incident upon the side of an assembly are $N x \approx 0.25 \mathrm{~g} / \mathrm{cm}^{2}$ and $\approx 0.15 \mathrm{~g} / \mathrm{cm}^{2}$, respectively.

These concentrations are used as reference values throughout the predictive modeling that is described in Sections 3 - 5. For consideration of different systems, the intensity of an NRF response may be scaled by $\mathrm{Pu}$ content to provide approximate results that may be used to estimate measurement times or detector requirements. Given that newer fuel cycles typically result in larger $\mathrm{Pu}$ concentrations, the use of these concentrations generally produce estimates that may be considered pessimistic.

\subsection{Instrumentation}

\subsubsection{Photon Sources}

Two types of photon sources are being considered for interrogation of spent nuclear fuel. Bremsstrahlung sources are available and provide the photon flux necessary to acquire necessary statistics 


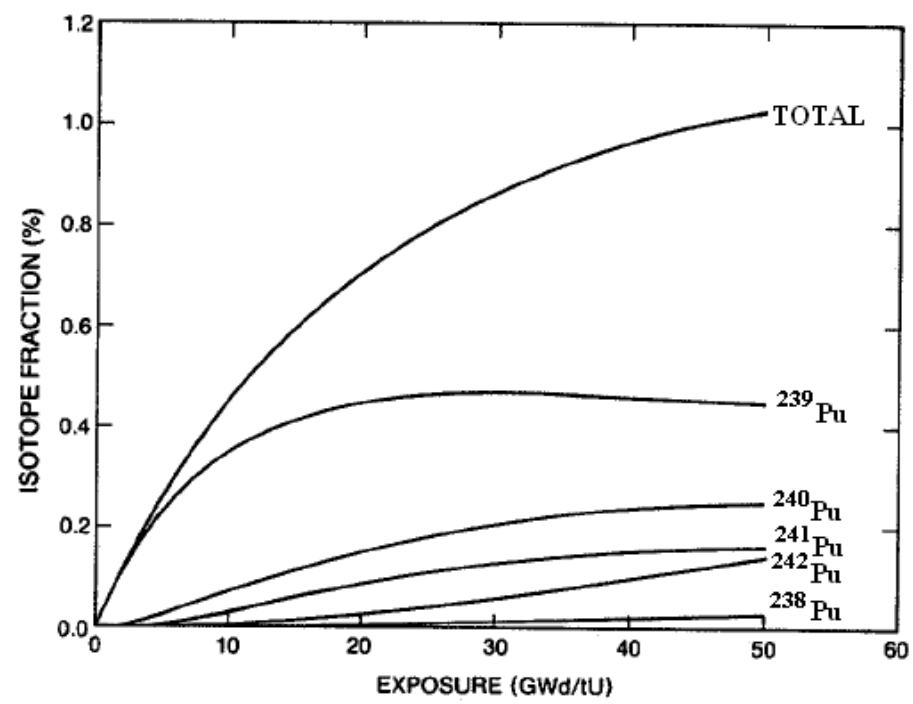

Figure 2.5 Example of computed Pu concentrations in spent fuel as a function of LWR reactor burn-up[9]. The figure is reproduced from this reference. The ordinate is percentage of $\mathrm{Pu}$ atoms to the initial uranium atoms present in the fuel.

for measurement of $\mathrm{Pu}$ in fuel assemblies. Quasi-monoenergetic photon sources offer great promise due to the possibility of very large increases in the signal-to-background ratio when compared to a bremsstrahlung source. However, quasi-monoenergetic sources are in the early stages of development and do not yet provide the necessary photon flux to precisely measure $\mathrm{Pu}$ content in spent fuel. Bremsstrahlung sources can, in principle generate continuous photon beams, referred to as CW sources, or can be operated in a pulsed mode, wherein the photons are generated in bunches. Today's quasi-monoenergetic sources operate in pulsed mode.

\section{Bremsstrahlung Sources}

Bremsstrahlung sources utilize electron accelerators to produce photons in a conversion target. Newer electron accelerators, such as rhodotrons, have reported currents approaching 100 mA[11, 12]. The energy spectrum of photons produced by a bremsstrahlung source is shown in Figure 2.6. Only a small fraction of photons that are produced are at the energies of resonances. A very large portion of the photons that are produced are at lower energies. These photons need to be filtered out in order to avoid saturating the $\gamma$-ray detectors.

Pulsed bremsstrahlung sources have also been studied as a potential method to induce NRF for non-destructive measurements. In principle, a pulsed system with excellent timing resolution and very rapid pulsing rates could use timing to discriminate against radioactive backgrounds, thereby reducing their importance. However, the discussion in Section 4.1 indicates that intense interrogating bremsstrahlung beams result in the majority of background photons being due to non-resonant scattering of beam photons, thereby reducing the importance of controlling the radioactive background.

The energy of the electron beam used to induce bremsstrahlung is a free parameter that must be optimized for a given measurement or system. Generally, higher endpoint energy bremsstrahlung beams result in higher count rates in detectors and larger background count rates. Conversely, higher endpoint energy beams excite a given resonance at a higher rate, thereby producing larger 


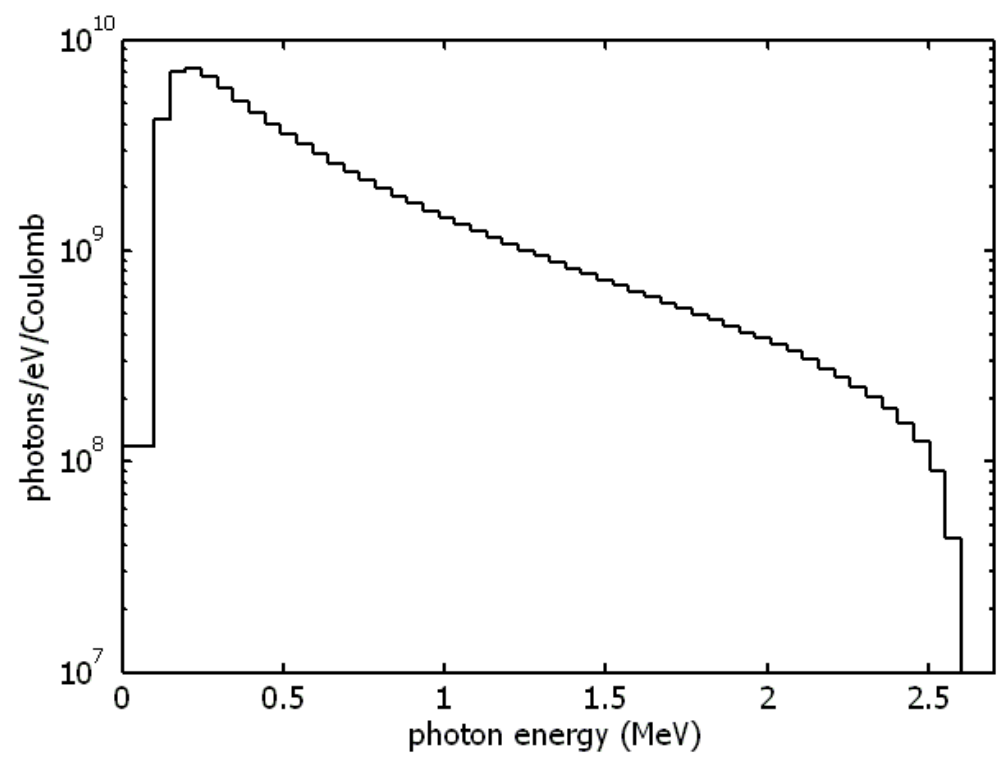

Figure 2.6 MCNPX-computed bremsstrahlung photons spectrum for photons leaving within $3.57^{\circ}$ of the initial electron trajectory, for $2.6 \mathrm{MeV}$ electrons normally incident upon $102 \mu \mathrm{m}$ thick Au foil backed by $1 \mathrm{~cm}$ thick $\mathrm{Cu}$.

Table 2.1 Laser Compton photon energies given by equation 2.21.

\begin{tabular}{ccccc}
\hline \hline $\begin{array}{c}E_{e^{-}} \\
(\mathrm{MeV})\end{array}$ & $\gamma$ & $\begin{array}{c}E_{L} \\
(\mathrm{eV})\end{array}$ & $\begin{array}{c}\theta \\
(\mathrm{mrad})\end{array}$ & $\begin{array}{c}E_{\gamma} \\
(\mathrm{keV})\end{array}$ \\
\hline 370.3 & 724.7 & 1.165 & 0.0 & 2431.5 \\
370.3 & 724.7 & 1.165 & 0.2 & 2381.8 \\
370.2 & 724.5 & 1.165 & 0.0 & 2430.2 \\
\hline 347.6 & 680.2 & 1.165 & 0.0 & 2143.6 \\
347.6 & 686.9 & 1.165 & 0.2 & 2104.7 \\
\hline 262.2 & 513.1 & 2.330 & 0.0 & 2431.5 \\
262.2 & 513.1 & 2.330 & 0.2 & 2406.4 \\
\hline 246.1 & 481.8 & 2.330 & 0.0 & 2143.6 \\
246.1 & 481.8 & 2.330 & 0.2 & 2125.5 \\
\hline
\end{tabular}

NRF count rates for a system. Although a formal optimization has not been performed, generally bremsstrahlung beams with endpoint energies that are a few hundred keV higher in energy than the resonance being measured have shown good results in experiments and simulations. More formal beam endpoint energy optimization should be considered when prototype NRF assay systems are being designed.

\section{Quasi-monoenergetic Sources}

The most advanced quasi-monoenergetic (QM) source type to be considered are laser-Compton photon sources. When a laser beam is scattered off a beam of relativistic electrons, the photons are up-shifted in energy to produce photons whose energies are given by[13, 14, 15, 16, 17]:

$$
E_{\gamma}=\frac{4 \gamma^{2} E_{L}}{1+(\gamma \theta)^{2}+4 \gamma E_{L} / m_{e} c^{2}}
$$


where $\theta$ is the angle between the electron beam and the up-scattered photon, in radians, $E_{L}$ is the laser energy, and $\gamma$ is given by

$$
\gamma=\frac{1}{\sqrt{1-\left(v_{e} / c\right)^{2}}}
$$

Table 2.1 presents evaluation of equation 2.21 assuming a Nd:YAG laser is used as the lowenergy photon source, and also for the case of a frequency-doubled Nd:YAG laser beam. Along with the required electron energies to produce resonant-energy photons, the strong angular dependence of photon beam energy is also shown. Using Nd:YAG laser energies, the electron energy must be adjustable between 335 and $375 \mathrm{MeV}$ to produce photons capable of exciting the known ${ }^{239} \mathrm{Pu}$ resonances. Likewise if the laser is frequency-doubled, the electron energy must be adjustable between 240 and $270 \mathrm{MeV}$.

Proposed facilities anticipate $1 \times 10^{6}$ photons $/ \mathrm{eV} / \mathrm{s}$ intensities and beam energy spreads as low as $1 \mathrm{keV}$. For quasi-monoenergetic photon systems under construction it is planned to produce QM photon sources utilizing $120 \mathrm{~Hz}, 10 \mathrm{ps}$ pulsed lasers[14]. The potential to time-correlate the counting of NRF $\gamma$ rays with the timing of the source may produce additional improvement in measurement parameters.

In Section 5, the importance of very intense photon sources will be made clear, and it will be explained that 10 - 100 times more intense photon sources than those currently proposed are needed for measurements of $\mathrm{Pu}$ content in spent fuel on a time scale of a few hours.

\subsection{2 $\gamma$-ray Detectors}

In order to use NRF to assay a material, the resonantly scattered photons must be observed against an intense background. Normally, this is best accomplished with high-resolution $\gamma$-ray detectors, however count rate requirements also play an important role in detector selection.

The best energy resolution for detectors widely used today is found with HPGe. While microcalorimeters have achieved better energy resolution, they have only been successful at measuring low-energy photons $(\lesssim 200 \mathrm{keV})$ at very low count rates $(\lesssim 1$ count/s)[18, 19]. Thus in this report, HPGe detectors are considered the base-line detector and other detectors capable of higher countrates are compared to $\mathrm{HPGe}$. At $2 \mathrm{MeV}$, HPGe energy resolution, $\Delta E$, is approximately $2.5 \mathrm{keV}$ (FWHM). Actual resolutions depend on the exact geometry of the Ge crystal, the bias voltage and the electronics used to readout the signal, but this value is used as representative of HPGe detector resolution.

HPGe and other detector types can vary significantly in geometries. To generally account for this, we assume that a reference detector will be a $100 \%$ relative efficiency HPGe detector, with a surface area of approximately $50 \mathrm{~cm}^{2}$. We conservatively assume that the count rate is limited to $20 \mathrm{kHz} .{ }^{3}$ An approximate drawing of such a detector in a typical shielding configuration is provided as Figure F.4.

To relate the relative intensities of NRF signals and backgrounds to the precision with which a measurement may be made, we present a simple statistical model. Suppose the signal of interest is detected at a rate, $S$. The background rate, $B$, is estimated by examining the count rate in adjacent channels. The total signal rate is, $T=S+B$. We define the signal-to-background ratio as $S / B=\xi$. After a counting period of $t$, we expect $t(S+B)$ total counts within the

\footnotetext{
${ }^{3} \mathrm{HPGe}$ detectors have operated above $100 \mathrm{kHz}$ but show distorted spectra. The optimization of detector rates could be considered in future work[20].
} 


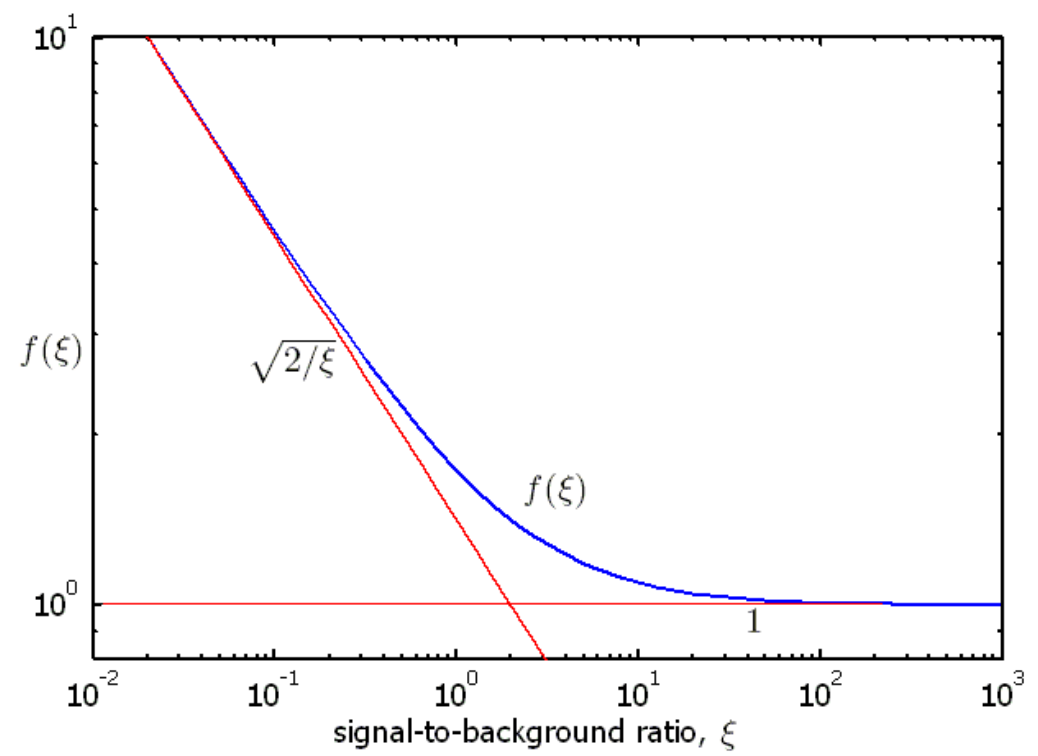

Figure 2.7 The relative increase in the fractional uncertainty of a measurement, $f(\xi)$ as a function of the signalto-background ratio, $\xi$, along with the functions (red) that describe $f(\xi)$ as $\xi \rightarrow 0$ and $\xi \rightarrow \inf$.

area of interest (presumably where an NRF peak is expected). The total number of counts from the signal is $N_{S}=t S$, with fractional uncertainty of $\vartheta=\frac{\sigma_{S}}{N_{S}}$. Assuming Poisson statistics, the standard deviation of the expected number of total counts, $N$, is $\sqrt{N}$, and we have

$$
\vartheta=\frac{\sqrt{t(T+B)}}{t S}=\frac{\sqrt{S+2 B}}{\sqrt{t} S}=\frac{\sqrt{1+2 / \xi}}{\sqrt{N_{S}}} \equiv \frac{f(\xi)}{\sqrt{N_{S}}}
$$

The function, $f(\xi)$, that expresses the reduction in statistical quality of a measurement of $N_{S}$ signal counts varies as $f \sim \sqrt{2 / \xi}$ for small values of $\xi$, whereas for large $\xi, f \rightarrow 1 . f(\xi)$ is shown in Figure 2.7 along with the limiting functions. This observation leads us to conclude that the NRF count rate must be almost as intense as the background signal rate within the detector's energy resolution to obtain counts with reasonable statistical quality.

For a given geometry, the count rates, $S$ and $B$ are both proportional to the detector's efficiency, $\epsilon$, therefore for a given measurement, $N \sim \epsilon$, and

$$
\vartheta \sim \frac{1}{\sqrt{\epsilon}}
$$

implying that high-efficiency detectors are preferred for their ability to increase $N_{S}$ for a given measurement.

Background signals due to processes such as elastic and inelastic scattering will vary slowly for photon sources that are broad in energy resolution compared to those of detectors. Therefore, using a HPGe detector, it is necessary that the NRF interaction rate be approximately 1000 $\left(\approx \Delta_{E} / \Gamma_{D}\right)$ times the scattering rate of non-resonant photons to accrue NRF count rate statistics in an efficient manner. This ratio increases proportionally with detector resolution.

As an example, a $\mathrm{LaBr}_{3}$ scintillation detector has a resolution that is about 18 times worse than a HPGe detector[21]. This implies that the background count rate within the detector's 
Table 2.2 Parameters relevant for Cherenkov detectors of multi-MeV photons

\begin{tabular}{lccc}
\hline \hline material & $n$ & $E_{t h}(\mathrm{MeV})$ & $E_{\gamma}(\mathrm{MeV})$ \\
\hline water & 1.333 & 0.262 & 0.262 \\
$\mathrm{SiO}_{2}$ nanotube film & 1.05 & 1.165 & 1.375 \\
$\mathrm{SF}_{6}$ gas @ 22 atm & 1.025 & 1.817 & 2.044 \\
$\mathrm{SiO}_{2}$ aerogel & 1.025 & 1.817 & 2.044 \\
$\mathrm{SF}_{6}$ gas @ 10 atm & 1.008 & 3.553 & 3.793 \\
$\mathrm{SF}_{6}$ gas @ 1 atm & 1.00078 & 12.41 & 12.66 \\
air @ 1 atm & 1.0003 & 20.6 & 20.8 \\
\hline
\end{tabular}

resolution will be about 18 times higher than it would be for an HPGe detector, and if $\xi_{\mathrm{HPGe}}=1$, the statistical value of a $\mathrm{LaBr}_{3}$ count,

$$
f\left(\xi_{\mathrm{HPGe}}\right) / f\left(\xi_{\mathrm{LaBr}_{3}}\right) \approx 1 / 3.5
$$

times less than that of an HPGe count. However, $\mathrm{LaBr}_{3}$ can operate at approximately 15 times higher count rates than HPGe, which may make its use advantageous, especially for transmission NRF measurements that use a TD.

\subsubsection{Threshold $\gamma$-ray Detectors}

The requirement that NRF detectors be capable of very high detection rates lead to investigation of alternative detector types, such as threshold detectors which only induce signals when incident particles have sufficient energy, such as Cherenkov detectors.[22, 23]

Cherenkov radiation is emitted when a particle such as an electron passes through a medium with a velocity, $v_{e}$, that is faster than the speed of light, $v_{h \nu}=c / n$ or if $\beta \equiv v_{e} / c>1 / n$. This gives a minimum electron energy of

$$
E_{t h}=m_{0} c^{2}\left(\sqrt{1+\frac{1}{n^{2}-1}}-1\right)
$$

Multi-MeV photons primarily interact by incoherent scattering or pair production. Electrons are primarily produced by incoherent scatter, yielding a continuum of electron energies up to $E_{e}=\frac{2 E_{\gamma}^{2}}{m_{e} c^{2}+2 E_{\gamma}^{2}}$. As $E_{\gamma}$ increases, this value nears $E_{\gamma}-m_{e} c^{2} / 2$. With $E_{\gamma}$ in the NRF energy range $(1.5-3 \mathrm{MeV})$, the difference between $\gamma$-ray energies and the most energetic Compton electrons is approximately $220 \mathrm{keV}$. Therefore in order to have some sensitivity for NRF photo-electrons, a Cherenkov detector must have $E_{t h}<E_{\gamma}-220 \mathrm{keV}$. Table 2.2 lists the index of refraction of many materials, the threshold energy for electrons to produce Cherenkov radiation, and the corresponding minimum photon energy that will induce Cherenkov radiation.

Since Cherenkov detectors do not directly measure photon energy, we consider their energydependent photon sensitivity. Each electron is capable of producing numerous Cherenkov photons, however the number of photons tends to be proportional to the energy of the electron inducing the Cherenkov emission. Examples of measured Cherenkov light yields are shown in Figure 2.8.

Because higher energy electrons induce larger signals, photons of higher energies than the resonance energy become particularly important as they contribute disproportionately to the measured 


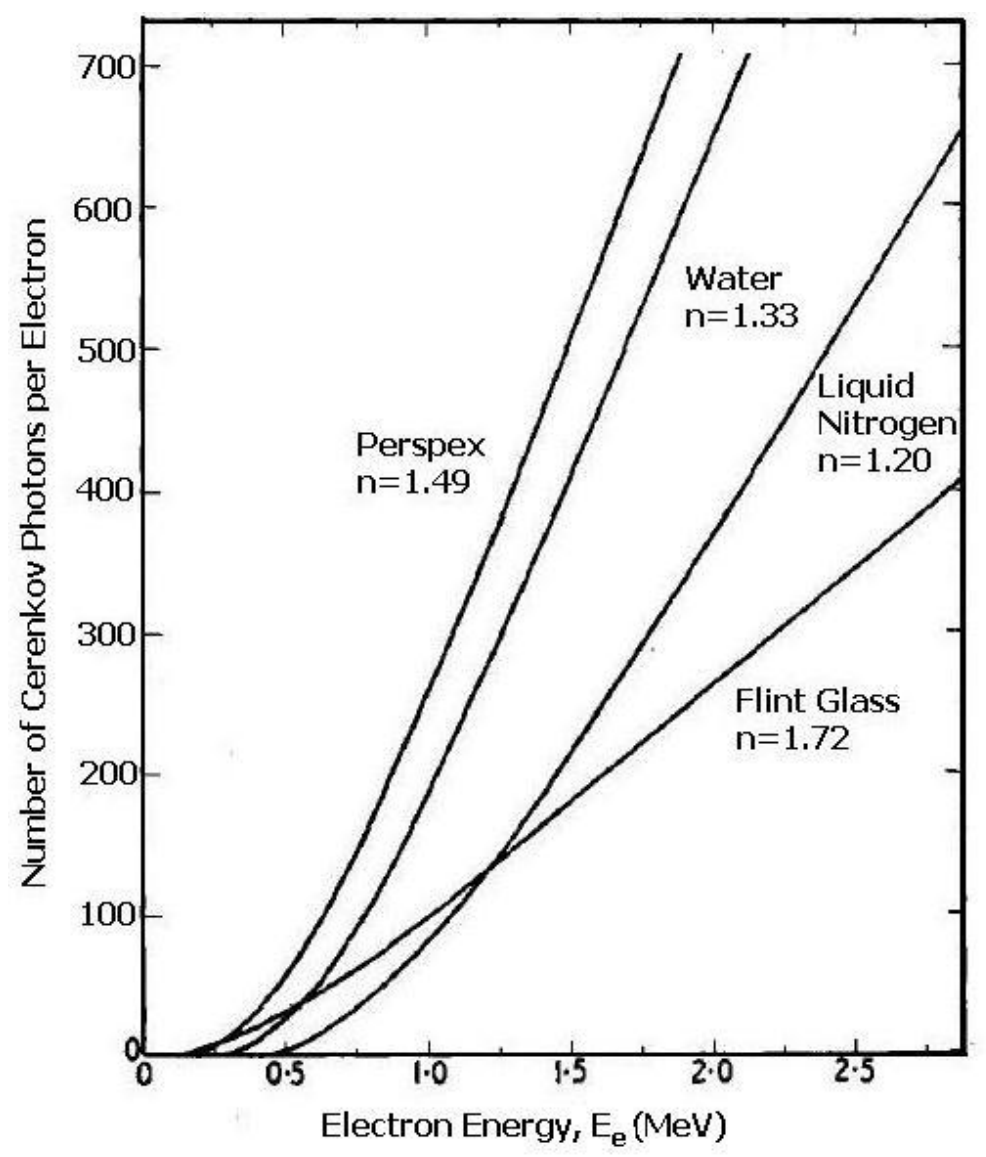

Figure 2.8 Measured Cherenkov light yields for electrons of energy, $E_{e}$ from Reference [23]. 
signal, which is problematic, especially for backscatter NRF measurements. We have found that these detector types would be useful in measurements conducted using quasi-monoenergetic photon sources that are approximately $10^{4}$ times more powerful than those currently being proposed. 


\section{Modeling - Analytical and MCNPX}

In this section the development and use of models to predict expected nuclear resonance fluorescence detection rates for non-destructive materials assay studies are discussed. These models assume that NRF is detected by a single radiation detector, and that use of multiple detectors would proportionally increase the calculated count rates. The two assay methods considered here, backscatter and transmission assay, have advantages and disadvantages that will be discussed in the following sections along with examples of measurements that have been modeled by a combination of computational and analytical methods.

\subsection{Backscatter Analytical Model}

A schematic drawing of a backscatter NRF assay geometry is shown in Figure 2.3. A source of energetic photons illuminates the target material (considered to be used fuel) and one or more radiation detectors measure the photon flux backscattered from the target. Section 2.3.2 indicates that improved detector energy resolution increases the statistical value of registered NRF counts. Because of this, high-purity germanium detectors (HPGe) are most commonly used for NRF experiments. The photon flux at the detector, $\Phi\left(\mathbf{r}_{\mathbf{d}}, E\right)$, which is proportional to the measured count rate, can be written as,

$$
\Phi\left(\mathbf{r}_{\mathbf{d}}, E\right)=\Phi_{\text {target }}\left(\mathbf{r}_{\mathbf{d}}, E\right)+\Phi_{\text {radioactivity }}\left(\mathbf{r}_{\mathbf{d}}, E\right)+\Phi_{\text {beam }}\left(\mathbf{r}_{\mathbf{d}}, E\right)
$$

where $\Phi_{\text {target }}\left(\mathbf{r}_{\mathbf{d}}, E\right)$ is the flux due to interrogating photons that were scattered toward the detector after interacting in the spent fuel, which includes the contribution due to NRF of constituent isotopes. $\Phi_{\text {radioactivity }}\left(\mathbf{r}_{\mathbf{d}}, E\right)$ is due to photons that were emitted due to radioactive decay of materials within the interrogation geometry, and $\Phi_{\text {beam }}\left(\mathbf{r}_{\mathbf{d}}, E\right)$ is due to interrogating beam photons that have reached the detector without interacting within the target material. Sufficient shielding must be placed between the bremsstrahlung source and the detectors to keep $\Phi_{\text {beam }}$ minimal.

\subsubsection{NRF Count Rates in Backscatter Assay}

We consider the contributions to $\Phi_{\text {target }}\left(\mathbf{r}_{\mathbf{d}}, E\right)$ due to resonant and non-resonant scattering separately. Resonant scattering produces NRF signals, whereas non-resonant scattering produces only background.

The rate at which NRF signals due to photons of energy, $E$, from a location, $\mathbf{r}$, within the target volume, $V$, are detected is given by:

$$
\frac{d^{2} R_{\mathrm{NRF}}}{d V d E}=N \Phi(E, \mathbf{r}) \sigma_{\mathrm{NRF}}(E) W_{e}(\theta) \exp \left[-\mu\left(E_{\gamma}\right) r_{o}\right]\left[\epsilon\left(E_{\gamma}\right) \frac{\Omega(\mathbf{r})}{4 \pi} P_{f}\left(E_{\gamma}\right)\right]
$$

where $N$ is the number density of atoms in the target that undergo NRF with cross section $\sigma_{\mathrm{NRF}}(E), \Phi(E, \mathbf{r})$ is the energy-differentiated photon flux at the point $\mathbf{r}, W_{e}(\theta)$ is the effective angular correlation function (approximately given by equation 2.8), $E_{\gamma}$ is the energy of the emitted $\mathrm{NRF} \gamma$ ray, which interacts within the target material with an attenuation coefficient, $\mu\left(E_{\gamma}\right)$ that 


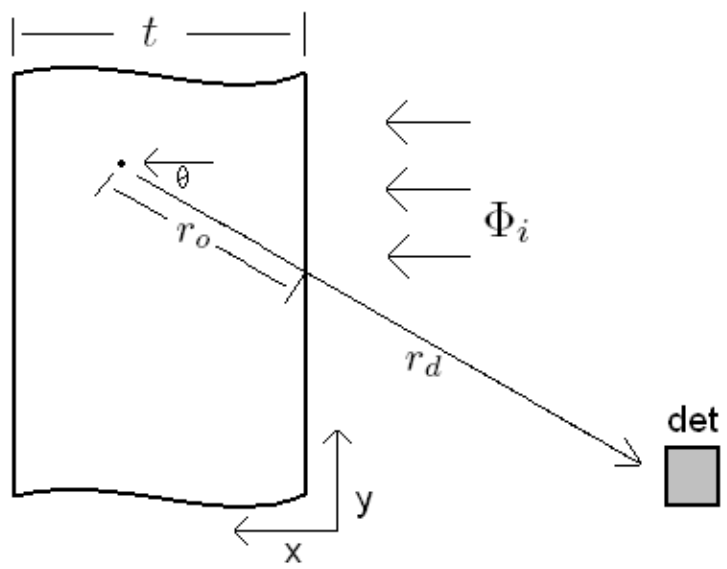

Figure 3.1 Schematic drawing of an NRF interrogation of a target slab.

results in a total attenuation of the $\mathrm{NRF} \gamma$ ray of $\exp \left[-\mu\left(E_{\gamma}\right) r_{o}\right], \epsilon\left(E_{\gamma}\right)$ is the probability that the radiation detector measures the full energy of the NRF $\gamma$ ray, $\frac{\Omega(\mathbf{r})}{4 \pi}$ is the fraction of the solid angle subtended by the radiation detector from the point where the $\gamma$ ray is emitted, and $P_{f}\left(E_{\gamma}\right)$ is the probability that the NRF $\gamma$ ray penetrates through the radiation filter without scatter.

NRF $\gamma$-ray emission corresponding to de-excitation to the nuclear ground state occurs with a probability $\Gamma_{0} / \Gamma$. Likewise emission to a different excited state, labeled $i$ would occur with a probability $\Gamma_{i} / \Gamma$. The resulting $\gamma$ ray energy is $E_{\gamma}=E-E_{\text {recoil }}$ where $E_{\text {recoil }}$ is given by equation 2.19. Generally, the energy transferred to the recoiling nucleus is sufficient to reduce the energy of the NRF $\gamma$ ray such that it is no longer resonant and attenuation of NRF $\gamma$ rays is only due to nonresonant interactions.

\section{Slab Geometry}

The simplest geometry to consider is a slab target of thickness, $t$, irradiated with a uniform parallel beam of intensity, $\Phi_{i}$, that is normally incident upon the slab. This geometry is applied to the calculation of the transmission detector response. A radiation detector is assumed to be located sufficiently far from the target that the beam diameter and $t$ are negligibly small compared to the detector distance, $r_{d}$. A schematic rendering of this geometry is shown in Figure 3.1. Although not indicated, the detector geometry may include a filter that NRF energy photons have a probability, $P_{f}\left(E_{\gamma}\right)$, to penetrate.

For simplicity, we neglect photon down-scatter. Then $\Phi(E, \mathbf{r})$ only varies due to the attenuation of photons:

$$
\Phi(E, x)=\Phi_{i} \exp [-\mu(E) x]
$$

and $\mu(E)$ now contains both resonant and non-resonant contributions:

$$
\mu(E)=\mu_{n r}+N \sigma_{\mathrm{NRF}}(E)
$$

Considering only photon energies near an NRF resonance, we may neglect the energy-dependence of the non-resonant attenuation coefficient, $\mu_{n r}$. Likewise, the attenuation coefficient for NRF $\gamma$ rays, $\mu\left(E_{\gamma}\right)$, is equal to $\mu_{n r}$ because nuclear recoil has made them non-resonant. 
The distance the photon must traverse to leave the target is given by $r_{o}=x / \cos (\theta)$, where $\theta$ is the angle between the interrogating photon trajectory and the direction of the detector's location. The detector's surface area is assumed to be given by $A$.

Substituting, the rate of detection of full-energy NRF $\gamma$ rays can be written as:

$$
\frac{d^{2} R_{\mathrm{NRF}}}{d x d E} \approx \exp \left[-\left(\mu_{n r}[1+1 / \cos (\theta)]+N \sigma_{\mathrm{NRF}}(E)\right) x\right] N \Phi_{i} \sigma_{\mathrm{NRF}}(E) W_{e}(\theta) \frac{A \epsilon P_{f} E_{\gamma}}{4 \pi r_{d}^{2}}
$$

We define

$$
\alpha=\mu_{n r}[1+1 / \cos (\theta)]
$$

and $\mu_{\mathrm{NRF}}(E)=N \sigma_{\mathrm{NRF}}(E)$ and obtain:

$$
\frac{d R_{\mathrm{NRF}}}{d E}=\int_{0}^{t} \frac{d^{2} R_{\mathrm{NRF}}}{d x d E} d x \approx \frac{1-\exp \left[-\left(\alpha+\mu_{\mathrm{NRF}}(E)\right) t\right]}{\alpha+\mu_{\mathrm{NRF}}(E)} N \Phi_{i} \sigma_{\mathrm{NRF}}(E) W_{e}(\theta) \frac{A \epsilon P_{f}\left(E_{\gamma}\right)}{4 \pi r_{d}^{2}}
$$

If we further approximate that

$$
\sigma_{\mathrm{NRF}}(E) \approx \begin{cases}0, & \text { if } E<E_{C}-\Gamma_{D} / 2 \\ \sigma_{\mathrm{NRF}}^{C}, & \text { if } E_{C}-\Gamma_{D} / 2 \leq E \leq E_{C}+\Gamma_{D} / 2 \\ 0, & \text { if } E>E_{C}+\Gamma_{D} / 2\end{cases}
$$

where

$$
\sigma_{\mathrm{NRF}}^{C}=\frac{\int \sigma_{\mathrm{NRF}}(E) d E}{\Gamma_{D}}
$$

$E_{C}$ is the centroid energy of the resonance, and $\Gamma_{D}$ is given by equation 2.14 . We may integrate equation 3.7 to obtain:

$$
R_{\mathrm{NRF}} \approx \frac{1-\exp \left[-\left(\alpha+N \sigma_{\mathrm{NRF}}^{C}\right) t\right]}{\alpha+N \sigma_{\mathrm{NRF}}^{C}}\left[N \Phi_{i} \sigma_{\mathrm{NRF}}^{C}\right] \frac{W_{e}(\theta) A \epsilon P_{f}\left(E_{\gamma}\right)}{4 \pi r_{d}^{2}}
$$

Equation 3.10 is arranged such that the expected rate at which NRF $\gamma$ rays are detected is divided into contributions due to three phenomena, the first term is due to the effective geometric attenuation of photons before and after NRF. A more complex derivation of this term for nontrivial geometries and use of a simple finite element integrating routine is described in Appendix $\mathrm{C}$. The second term, $N \Phi_{i} \sigma_{\mathrm{NRF}}^{C}$ is the rate (per unit thickness) at which NRF would occur in the target without any attenuation, and the final term is due to the probability of detection of NRF $\gamma$ rays emitted from the target.

Although the constant cross section approximation of equation 3.8 will prove to be very flawed in analysis of transmission assay, the assumption that the solid angle subtended by the detector is independent of the interaction location within the target proves to be the largest approximation in many backscatter NRF assay geometries.

\subsection{Transmission Model}

\subsubsection{Transmission Assay: NRF and Background Signal Rates}

Photons penetrating the fuel assembly undergo attenuation given by

$$
\Phi(E)=\Phi_{0}(E) \exp [-\mu(E) x]
$$


where $x$ is the thickness of assembly material the photon penetrates, and $\mu(E)$ is the energydependent attenuation coefficient for photons in the assembly material, given by

$$
\mu(E)=\sum_{i j} N_{i} \sigma_{i j}(E)
$$

where $N_{i}$ is the number density of a given isotope, $i$, and $\sigma_{i j}(E)$ is the partial cross section for a photon interaction event of type $j$ for that isotope (i.e., incoherent scattering, pair production, $\mathrm{NRF}, \ldots)$. There is also the potential for higher-energy photons to be down-scattered to the energy, $E$, resulting in higher photon fluences than indicated by equation 3.11. The phenomenon of notch refill, where a down-scattered photon becomes resonant in energy, is discussed in more detail in Section 3.3.4. However, for the case where the energy of an NRF resonance is near the maximum energy of the interrogating photon spectrum, this occurrence is relatively rare whereas, downscattering significantly increases the photon flux for the low-energy portion of the transmitted spectrum, relative to that predicted by equation 3.11 .

The processes by which non-resonant photons are backscattered to produce background events will be described in some detail in Section 4.1 when the backscatter measurement is examined. Whereas the target described in Section 4.1 is the assay target, the same physics apply to the TD and therefore similar calculations are performed for the TD. The primary difference being the fuel assembly hardens the spectrum of the interrogating beam, and the shielding requirements for the radiation detectors are significantly reduced.

We consider non-resonant photon transport through a target composed of $\mathrm{UO}_{2}$, $\mathrm{Zr}$, and small quantities of other actinides and fission products. The target geometry is a homogenized mixture of these constituents at $4 \mathrm{~g} / \mathrm{cm}^{3}$ density and $21.8 \mathrm{~cm}$ square, which is the homogenized equivalent of a 15 pin by 15 pin $(15 \times 15)$ spent fuel assembly or, due to thinner pins, it is also similar to the 17x17 assembly considered in Appendix B. Expected photon intensities are estimated with MCNPX calculations, where the effects of resonant absorption are explicitly excluded. Resonant absorption is calculated separately using the formalisms developed in Section 3.2.2.

The bremsstrahlung spectrum used in this simulation to irradiate the target is that described in Section 2.3.1. This spectrum, shown in Figure 2.6 is assumed to be produced $150 \mathrm{~cm}$ from the side face of the $15 \times 15$ homogenized fuel assembly target. The geometry is such that the diameter of the un-scattered beam is $21.8 \mathrm{~cm}$ at the back plane of the target. The spectrum of photons leaving the target's back plane in the direction of the TD is shown in Figure 3.2. The integrated spectrum is attenuated by a factor of $\sim 400$; however, photons above $2 \mathrm{MeV}$ are only attenuated by a factor of $40-50$.

A third computation simulated the transport of photons with the spectrum shown in Figure 3.2 as they impinge upon the TD. The energy-differentiated photon flux was calculated at a point 100 $\mathrm{cm}$ from the TD at an angle of $120^{\circ}$ relative to the initial beam direction. This photon energy distribution is then taken as the source spectrum for a final series of simulations in which the shielded detector response is examined. In these simulations, the thickness of the $\mathrm{Pb}$ filter was varied, and the backscattered photon spectrum was transported through a shielded HPGe detector geometry as described in Section F.4. For each incident photon, the energy deposited within the germanium was calculated, resulting in the expected photon spectrum as measured by a shielded HPGe detector.

The thicknesses of the $\mathrm{Pb}$ filters were 1.27, 2.54, 4, 6, and $8 \mathrm{~cm}$ for these simulations. Increasing the filter thickness from $1.27 \mathrm{~cm}$ to $8 \mathrm{~cm}$ decreases the total count rate in the detector $(100 \%$ 


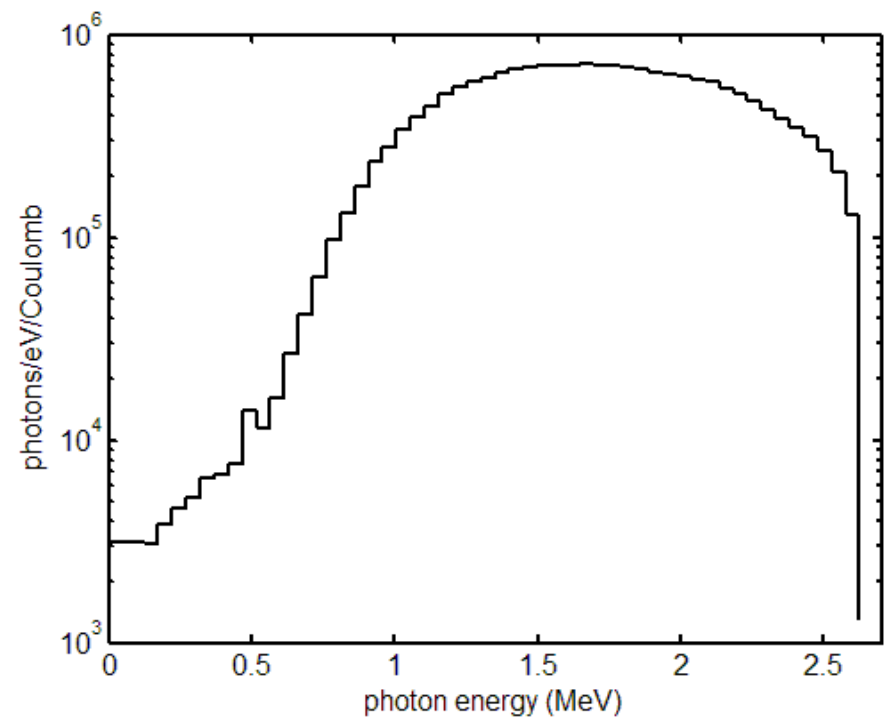

Figure 3.2 Calculated spectrum of photons leaving a fuel assembly rotated $33^{\circ}$ in the direction of a TD located 105 $\mathrm{cm}$ downstream from the assembly. The incident photon spectrum was $2.6 \mathrm{MeV}$ endpoint energy bremsstrahlung, similar to that shown in Figure 2.6, except the source collimation was assumed to be $1^{\circ}$.

relative efficiency HPGe) from $4.4 \times 10^{7}$ to $1.2 \times 10^{5}$ counts per Coulomb of $2.6 \mathrm{MeV}$ electrons incident upon the bremsstrahlung converter. In general, an HPGe detector should operate at a maximum of between $2 \times 10^{4}$ and $8 \times 10^{4} \mathrm{~Hz}[24]$. In the analyses reported here we use $2 \times 10^{4}$ as a conservative assumption for the maximum count rate although insignificantly higher rates may be possible. The maximum count rate defines the maximum allowable beam current for a given filter thickness. These values are summarized in Table 3.1.

Increasing the filter thickness results in more attenuation of the NRF $\gamma$ rays. This decreases the probability that a NRF $\gamma$ ray will deposit its full-energy in the HPGe detector. This attenuation is estimated with the expression $I=I_{O} \exp \left(-\mu x_{f}\right)$, where $\mu$ is the attenuation coefficient (without coherent scattering) taken from reference[25]. The attenuation due to the filter, $I_{\gamma} / I_{\gamma 0}$ is also shown in Table 3.1 for a $2.25 \mathrm{MeV} \gamma$ ray. Attenuation coefficients are not a strong function of energy in the range between 2 and $2.5 \mathrm{MeV}$, and therefore we can consider these values to be fairly representative of the behavior NRF $\gamma$ rays that would be induced by a $2.6 \mathrm{MeV}$ endpoint-energy bremsstrahlung beam.

Neglecting the excess attenuation of resonant-energy photons (which will be explicitly examined in detail in Section 3.2.2), the rate at which NRF $\gamma$ rays are detected can be estimated using equation 3.10, where $\Phi_{i}$ now refers to the photon spectrum incident upon the TD (Figure 3.2), and the full-energy photon detection efficiency, $\epsilon$, must also take into account the filtering effect. With these modifications, we show in the second column of Table 3.1 expected count rates of a hypothetical $20 \mathrm{eV} \cdot \mathrm{b}$ resonance at $2.25 \mathrm{MeV}$ for a $100 \%$ relative efficiency HPGe detector located $100 \mathrm{~cm}$ from the TD per Coulomb of electrons incident upon the bremsstrahlung converter.

We then examine how the expected rate at which NRF $\gamma$ rays are measured decreases due to resonant absorption of photons in the assay target. This measured decrease in the intensity of transmitted resonant-energy photons is used to determine the areal density of the IOI in the target. 
Table 3.1 Effects of the photon filter on the photon spectrum that is calculated to be measured by a $100 \%$ relative efficiency HPGe detector. $x_{\mathrm{f}}$ indicates the thickness of the Pb filter. The values corresponding to counts per 2.6 $\mathrm{MeV} \mathrm{e} \mathrm{e}^{-}$indicate the total number of expected counts per $2.6 \mathrm{MeV}$ electron incident upon the bremsstrahlung converter, and from these values, the electron beam intensity resulting in $2 \times 10^{4}$ counts per second, $I_{\max }$, is calculated. Values labeled as $I_{\gamma} / I_{\gamma 0}$ indicate the attenuation of a $2.25 \mathrm{MeV} \gamma$ ray as it penetrates through the corresponding filter thickness.

\begin{tabular}{ccrr}
\hline \hline $\begin{array}{c}x_{\mathrm{f}} \\
(\mathrm{cm})\end{array}$ & $\begin{array}{c}\text { counts per } \\
2.6 \mathrm{MeV} \mathrm{e}^{-}\end{array}$ & $\begin{array}{r}I_{\max } \\
(\mathrm{mA})\end{array}$ & $I_{\gamma} / I_{\gamma 0}$ \\
\hline 1.27 & $7.0 \times 10^{-12}$ & 0.46 & 0.53 \\
2.54 & $1.8 \times 10^{-12}$ & 1.8 & 0.28 \\
4.0 & $4.1 \times 10^{-13}$ & 7.8 & 0.14 \\
6.0 & $7.6 \times 10^{-14}$ & 42 & 0.051 \\
8.0 & $1.9 \times 10^{-14}$ & 170 & 0.019 \\
\hline
\end{tabular}

\subsubsection{Analytical Considerations}

In this section, we introduce the analytical model that relates the areal density of a $\mathrm{Pu}$ isotope to the decrease in the intensity of the NRF $\gamma$ rays measured by radiation detectors in the transmission geometry. We consider the relative attenuation of photons at, and near, resonant-energies through materials containing varying amounts of an IOI. As discussed in Section 2.1.2, the energydependent cross section that photons experience while traversing a material is given to an excellent approximation by the Doppler-broadened Lorentzian profile (DBLP) described in equation 2.16. The relative probability that a photon at or near resonant-energy traverses the assay target and then subsequently undergoes NRF in the TD is considered. In Section 3.2.4 the expected NRF probabilities are compared to alternate predictions that would result if approximate models of the energy-dependent cross section were used.

First, we assert that within a few eV of a resonance, it is valid to approximate all non-resonant cross sections as constants. This approximation is accurate to a fraction of a percent[25]. Likewise, across the width of a resonance, the change in intensity of bremsstrahlung-spectrum photons may be neglected. With this, the energy-differentiated photon flux, $\Phi_{i}(E)$, incident upon the assay target is assumed constant and effects due to non-resonant photon attenuation are separated. Neglecting down-scattering, the effective photon flux leaving a target is approximately

$$
\Phi_{o}(E) \approx \Phi_{i} \exp \left[-N \sigma_{\mathrm{NRF}}(E) x\right] \exp \left[-\mu_{\text {atom }} x\right]
$$

where $N$ is the atom density of a $\mathrm{Pu}$ isotope (in units of atoms $/ \mathrm{cm}^{3}$ ) in the assembly, $x$ is the target thickness (that is determined by a ray-tracing algorithm, see Section 3.2.3), $\sigma_{\mathrm{NRF}}(E)$ is the cross section due to NRF, and $\mu_{\text {atom }}$ is the attenuation coefficient for photons in the assay target due to non-resonant processes (assumed to be constant over the energy range of interest).

The rate at which NRF $\gamma$ rays emitted into backward angles relative to the direction of the $\mathrm{NRF}$-inducing incident beam are counted in a geometry consisting of a slab target was considered in Section 3.1.1. The only difference here is that the photon flux incident upon the slab is now $\Phi_{o}(E)$.

$$
\frac{d R_{\mathrm{NRF}}}{d E} \approx \frac{1-\exp \left[-\left(\alpha+\mu_{\mathrm{NRF}}(E)\right) t_{\mathrm{TD}}\right]}{\alpha+\mu_{\mathrm{NRF}}(E)} N_{\mathrm{TD}} \Phi_{o}(E) \sigma_{\mathrm{NRF}}(E) W_{e}(\theta) \frac{A \epsilon P_{f}\left(E_{\gamma}\right)}{4 \pi r_{d}^{2}}
$$


where $t_{\mathrm{TD}}$ refers to the TD thickness and $\alpha$ is defined in equation 3.6.

Equation 3.14 may be re-written as follows

$$
\frac{d R_{\mathrm{NRF}}}{d E}=\lambda(E)\left[\exp \left[-N \sigma_{\mathrm{NRF}}(E) x\right] \sigma_{\mathrm{NRF}}(E)\right]\left[N_{\mathrm{TD}} \Phi_{i} \exp (-\mu x) W_{e}(\theta) \frac{A \epsilon P_{f}\left(E_{\gamma}\right)}{4 \pi r_{d}^{2}}\right]
$$

The first term,

$$
\lambda\left(E, t_{\mathrm{TD}}\right)=\frac{1-\exp \left[-\left(\alpha+\mu_{\mathrm{NRF}}(E)\right) t_{\mathrm{TD}}\right]}{\alpha+\mu_{\mathrm{NRF}}(E)}
$$

is an effective thickness that a photon experiences as it traverses the slab. If the quantity $(\alpha+$ $\left.\mu_{\mathrm{NRF}}(E)\right)$ is small,

$$
\lambda\left(E, t_{\mathrm{TD}}\right) \approx t_{\mathrm{TD}}
$$

Indicating that the rate of $\mathrm{NRF} \gamma$ ray detection would be directly proportional to the TD thickness. However, for geometries containing a thick TD or if larger photon interaction probabilities are present, the rate at which NRF is induced in the slab is less than $N_{\mathrm{TD}} \sigma_{\mathrm{NRF}} t_{\mathrm{TD}}$ because $\lambda(E) \leq t_{\mathrm{TD}}$.

We define the first two terms of equation 3.15 as $\Lambda\left(E, t_{\mathrm{TD}}, N x\right)$,

$$
\Lambda\left(E, t_{\mathrm{TD}}, N x\right) \equiv \lambda(E)\left(\exp \left[-N \sigma_{\mathrm{NRF}}(E) x\right] \sigma_{\mathrm{NRF}}(E)\right)
$$

This function contains all the parameters that can significantly vary over an energy range comparable to the Doppler-broadened width of an NRF resonance. The energy resolutions of all commonly used $\gamma$-ray detectors are much wider than the width of an NRF resonance. Therefore energy-dependent variations in $\Lambda$ will not be directly observed in the detected photon energy spectrum and the quantity $\int \Lambda(E) d E$ provides a quantity that is proportional to the rate at which NRF counts will be measured.

The measured quantity that provides information on the areal density of the Pu isotope in the sensitive volume of the assay target is the reduced rate of NRF $\gamma$-ray counts, relative to those expected for a comparable assay target with none of the $\mathrm{Pu}$ isotope present. This quantity is called the effective attenuation and is given by

$$
\mathbb{A}\left(t_{\mathrm{TD}}, N x\right) \equiv \frac{\int \Lambda\left(E, t_{\mathrm{TD}}, N x\right) d E}{\int \Lambda\left(E, t_{\mathrm{TD}}, 0\right) d E}
$$

The effective attenuation is dependent upon the parameters of the resonance it describes. Effective attenuation functions are shown for different ${ }^{239} \mathrm{Pu}$ resonances when $t_{\mathrm{TD}}=8 \mathrm{~g} / \mathrm{cm}^{2}$ in Figure 3.3. The parameters of the known ${ }^{239} \mathrm{Pu}$ NRF resonances are shown in Table 3.3. Figure 3.3 indicates that for a target of constant total areal density, but increasing $\mathrm{Pu}$ areal density, NRF $\gamma$-ray count rates decrease. The rate at which they decrease is proportional to the width of the resonance. By observing this reduced rate, the areal density of the $\mathrm{Pu}$ isotope is measured.

$\Lambda\left(E, t_{\mathrm{TD}}, N x\right)$ and therefore $\mathbb{A}\left(t_{\mathrm{TD}}, N x\right)$ are functions of $t_{\mathrm{TD}}$. The thin TD sheet approximation can result in significant errors $\left(30 \%\right.$ of $\left.\mathbb{A}\left(t_{\mathrm{TD}}, N x\right)\right)$ when a thick TD is used to measure targets with significant resonant attenuation. These effects however, appear predictable and are less severe when $\mathbb{A}\left(t_{\mathrm{TD}}, N x\right)$ is nearer unity. 


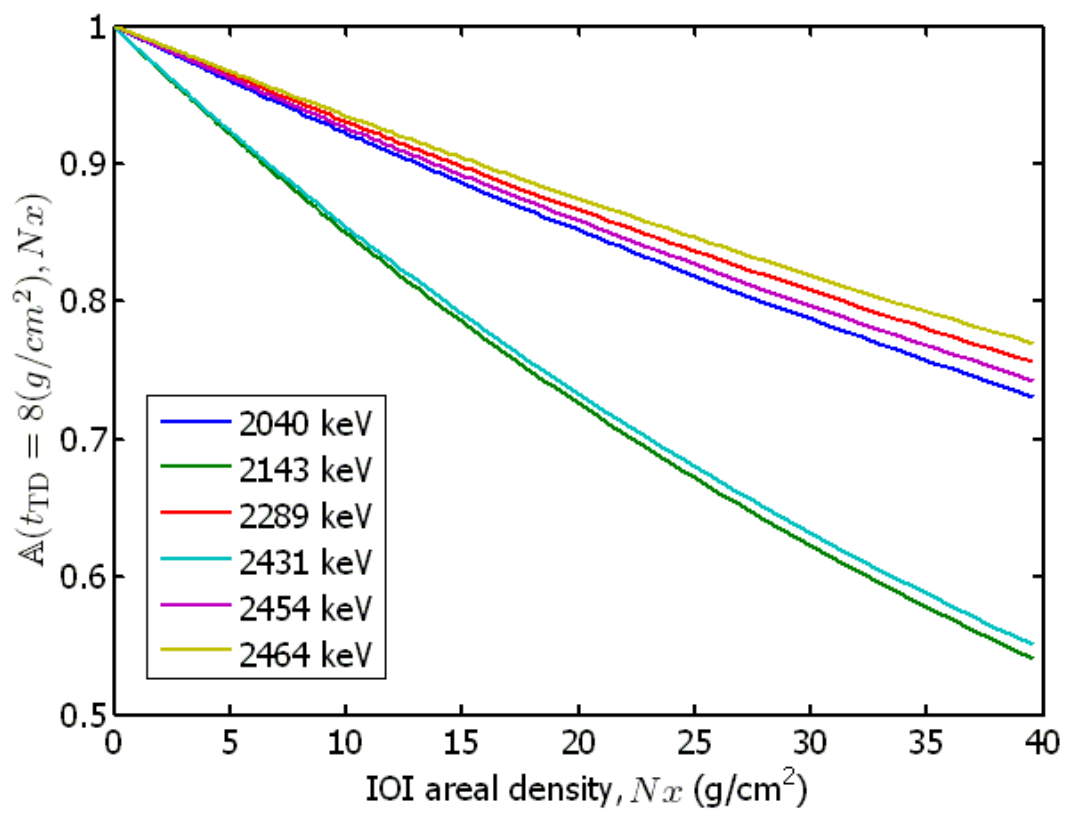

Figure 3.3 The effective attenuation of photons given by equation 3.19 due to ${ }^{239} \mathrm{Pu}$ NRF resonances with $t_{\mathrm{TD}}=8$ $\mathrm{g} / \mathrm{cm}^{2}$. Data for the resonances are shown in Table 3.3 and are from reference [26]. Each resonance is indicated by its centroid energy in the legend.

\subsubsection{Ray-Tracing Algorithm}

A ray-tracing algorithm was written to compute the linear distance of fuel that a source photon would traverse as it penetrates a fuel assembly. This value is used as $x$ in the resonant attenuation expression, equation 3.19. This process relates realistic non-homogeneous structures, such as fuel assemblies to the 1-dimensional thicknesses that have been assumed in the analytical model.

The ray-tracing algorithm was tested on a $17 \times 17$ fuel assembly geometry, described in Appendix B, with MCNPX using F4 tally values in which the contents of constituent volume cells are replaced with void. The MCNPX simulatation produces lengths of segments of fuel pins through which particles traverse without interacting, which is what the ray-tracing algorithm was written to predict. Comparing the calculations for identical geometries produced identical results.

The ray-tracing algorithm was used to determine a source and assembly configuration in which a collimated photon source would produce the smallest variations in $x$, regardless of the direction within the collimator opening angle the photon was emitted. The geometry was varied between ray-tracing simulations by rotating the fuel assembly around the assembly's lengthwise axis, by approximately $2^{\circ}$ increments. To maintain consistency between simulations, the maximum allowed divergence of the bremsstrahlung beam was determined such that all rays must enter one plane and exit the opposite plane (i.e., no rays would enter the assembly in one plane and leave in an adjacent plane). Two views of an example ray-tracing geometry are shown in Figures 3.4 and 3.5. The opening angle of the beam linearly decreases from about $5^{\circ}$ to $1^{\circ}$ as the assembly rotation angle increases from $5^{\circ}$ to $36^{\circ}$.

Using this ray-tracing routine, we produced histograms of the linear fuel distance through which incident photons would penetrate for a given angle of assembly rotations. Figures 3.6 and 3.7 are two such histograms for geometries in which the bremsstrahlung source is assumed to be $100 \mathrm{~cm}$ from the nearest point of the fuel assembly, and the fuel assembly rotation angles are $29.08^{\circ}$ and 


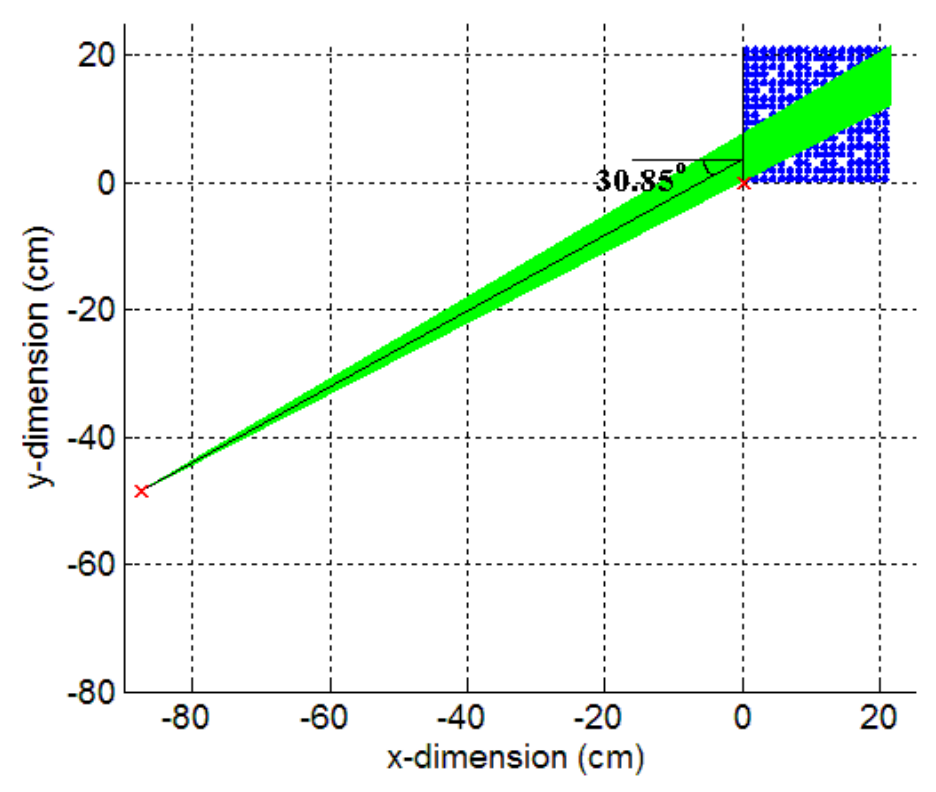

Figure 3.4 Top-view of a ray-tracing geometry. In this simulation, the minimum distance between the source and assembly (the two locations are indicated by red x's) is $100 \mathrm{~cm}$. The mean incident angle is $30.85^{\circ}$. The fuel assembly is shown as the blue structure at the top-right, and each green line indicates a ray.

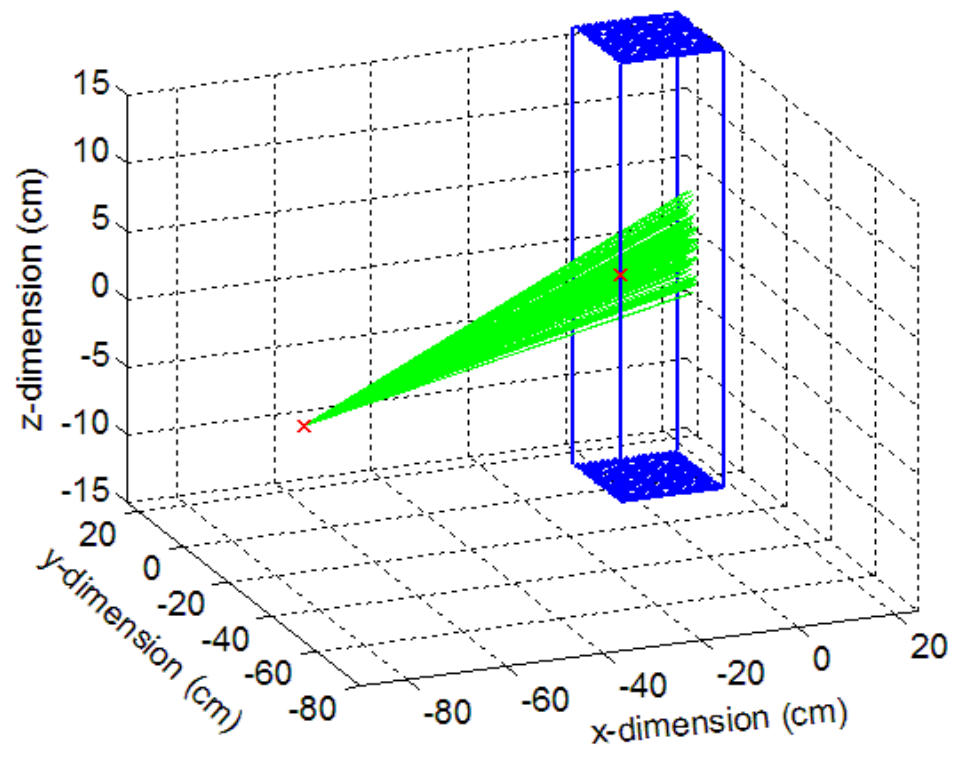

Figure 3.5 Perspective view of the ray-tracing geometry shown in Figure 3.4. Note the z dimension is stretched relative to $\mathrm{x}$ and $\mathrm{y}$. 


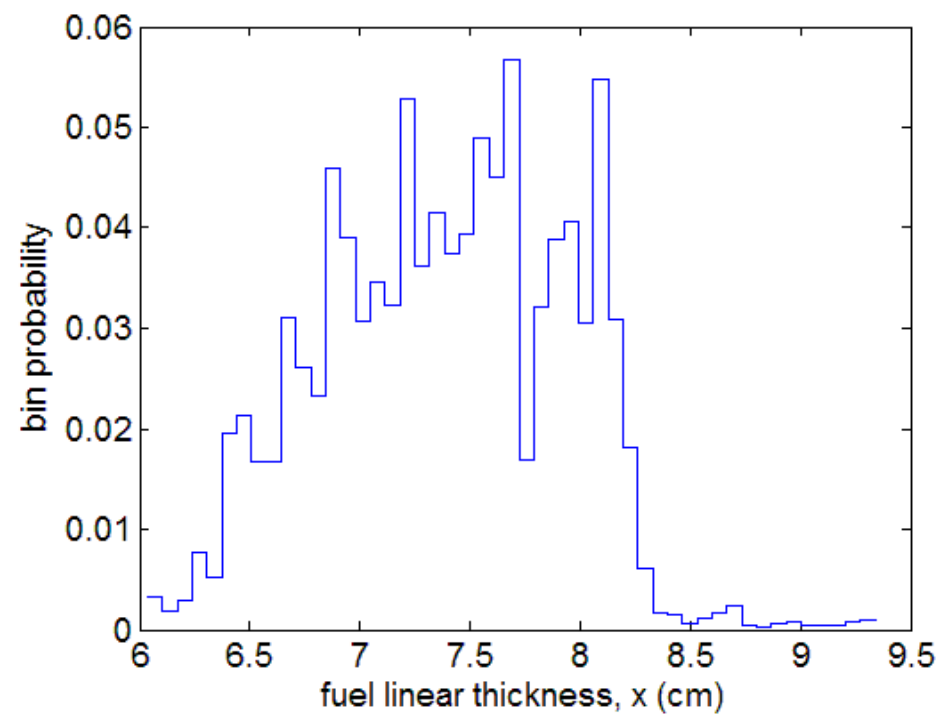

Figure 3.6 Histogram of fuel thicknesses through which photons penetrate for $100 \mathrm{~cm}$ distance source, with a $2.08^{\circ}$ half-angle collimator, and fuel assembly rotation of $29.08^{\circ}$. The $x$ bin widths are $0.67 \mathrm{~mm}$.

$30.85^{\circ}$, respectively.

Comparing Figures 3.6 and 3.7, we note that the width of the distribution of thicknesses through which photons incident upon a $29.08^{\circ}$ rotated assembly is much wider than that for the $30.85^{\circ}$ rotation. Values of the mean linear distance through fuel, $\bar{x}$ penetrated versus rotation angle are shown in Figure 3.8 for the $100 \mathrm{~cm}$ source distance. In this figure, the error bars indicate the standard deviation in the distance values, $\sigma_{x}$. These standard deviation values are due to the geometry of the problem, and not statistics. The ratios of the standard deviations to the mean values, $\sigma_{x} / \bar{x}$, are shown in Figure 3.9 for both $100 \mathrm{~cm}$ and $200 \mathrm{~cm}$ source distances. Considering the two curves, we observe that the smallest fractional change in fuel widths penetrated occurs with rotation angles near 30 or $38^{\circ}$, and that the differences between $100 \mathrm{~cm}$ and $200 \mathrm{~cm}$ source distances are not significant.

\subsubsection{Approximate NRF Cross Section Models}

In the previous section assumed that all resonances take the Doppler-Broadened Lorentzian Profile (DBLP) given in equation 2.16. However, approximate forms of the DBLP are often assumed. The NRF data added to MCNPX and ENDF files as described in Section 3.3.1 uses one such approximation.

The forms considered for resonance shapes are:

- a Maxwell-Boltzmann profile of equation 2.12 with width, $\Delta$, given by equation 2.13;

- a point-wise evaluation of the Maxwell-Boltzmann form with linear interpolation between points. The energies at which the Maxwell-Boltzmann distribution was evaluated are the centroid energy, $E_{C}$, and $E_{C} \pm 2 \mathrm{eV}$ and $E_{C} \pm 4 \mathrm{eV}$; and

- a step increase and decrease as defined in equation 3.8 . 


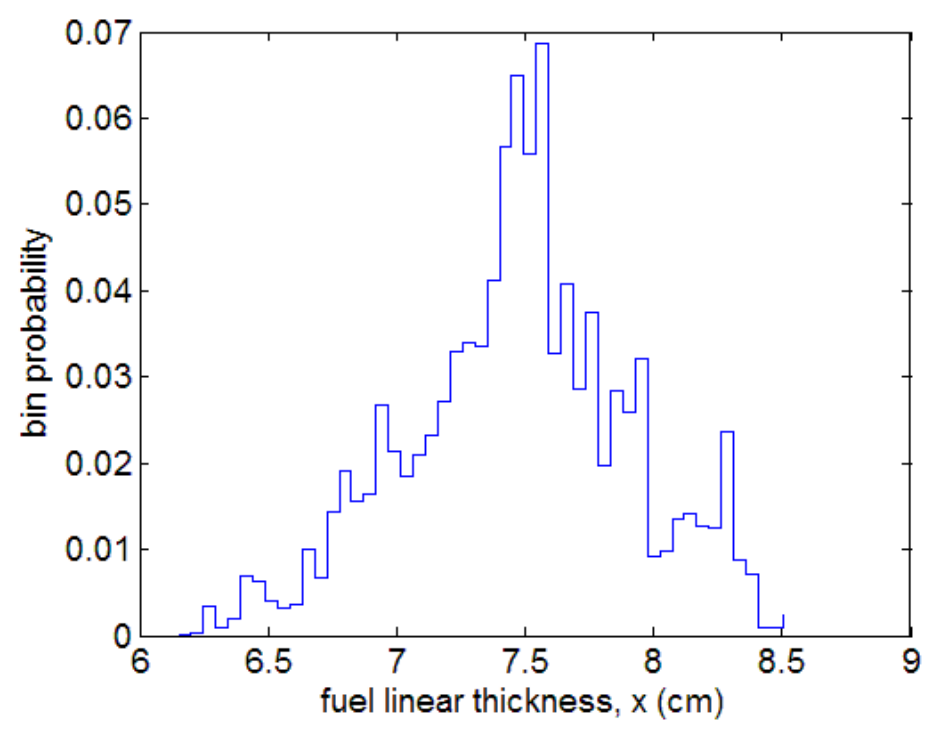

Figure 3.7 Same as Figure 3.6, but for $1.85^{\circ}$ half-angle collimator, $30.85^{\circ}$ fuel assembly rotation, and $0.48 \mathrm{~mm}$ bin widths.

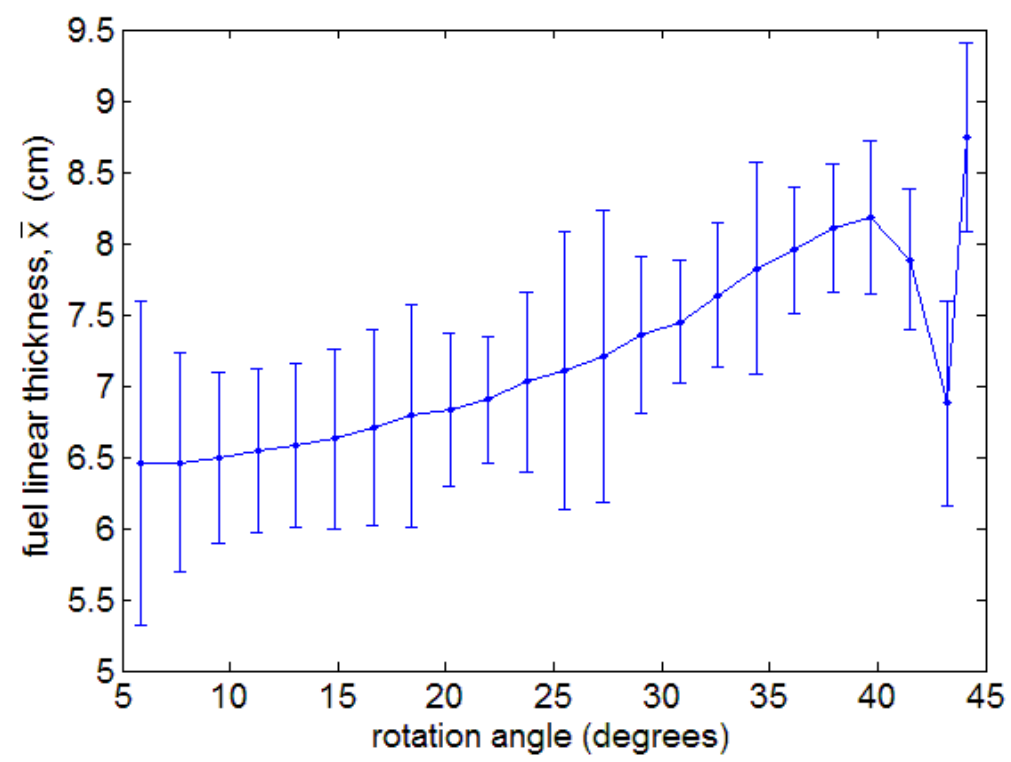

Figure 3.8 Mean values of the linear thickness of fuel, $\bar{x}$, that a ray traverses versus assembly rotation angle for typical 17x17 PWR assembly. The source of photons is assumed to be a point source at $100 \mathrm{~cm}$ distance, collimated such that the photons must pass from the front plane to the back plane of the assembly as indicated in Figures 3.4 and 3.5. The vertical error bars indicate the standard deviation of the fuel lengths traversed by the rays, $\sigma_{x}$. 


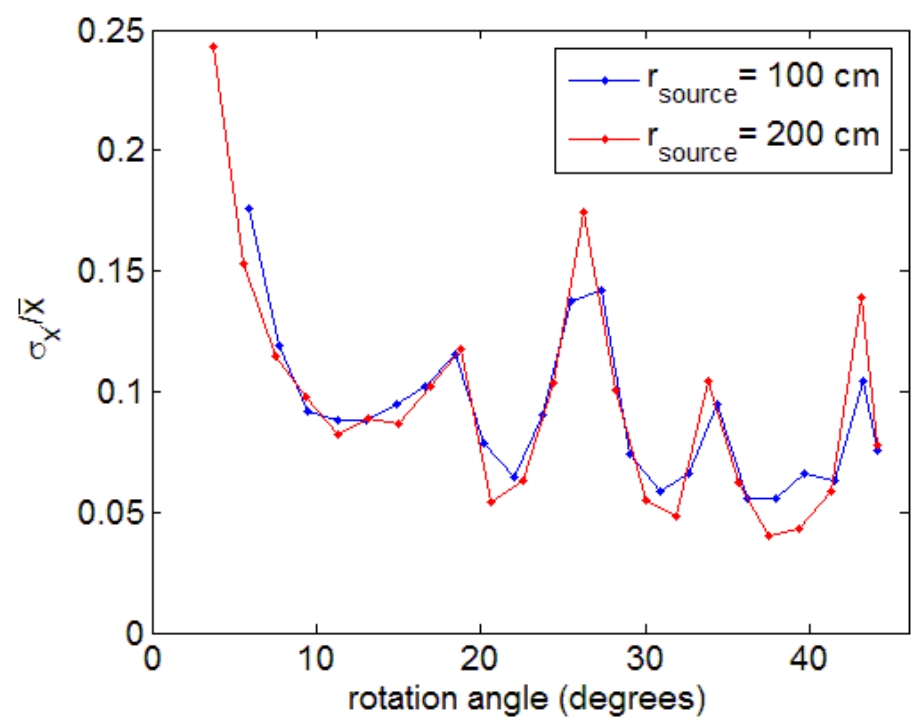

Figure 3.9 The standard deviation, of fuel lengths traversed by rays divided by the mean length versus fuel assembly rotation for $100 \mathrm{~cm}$ and $200 \mathrm{~cm}$ source distances.

The cross section from each model is normalized such that the integrated cross section is the same as that of the natural cross section. These functional forms with shape parameters due to the $2209 \mathrm{keV}$ resonance of ${ }^{238} \mathrm{U}[27]$ are shown in Figure 3.10 .

The differing approximate forms of the cross section profile result in calculated values of $\mathbb{A}\left(t_{\mathrm{TD}}, N x\right)$ that may differ by up to $5 \%$ for ${ }^{239} \mathrm{Pu}$ isotopic areal density found in spent fuel. The largest disagreement results from the use of the constant cross section approximation. Use of the Maxwell-Boltzmann profile or its point-wise evaluation will generally produce less than $1 \%$ errors for ${ }^{239} \mathrm{Pu}$ areal densities. However, the transmission experiment described in Appendix A involved significantly larger areal densities, and therefore careful consideration of the forms of the resonances was necessary.

\subsubsection{Relating Attenuation Measurement to Pu Content in Spent Fuel}

In this section, we examine the precision at which the actinide content may be measured in spent fuel by way of a transmission NRF measurement. First, the range in which $\mathrm{Pu}$ concentrations are expected in spent fuel will be discussed, followed by a statistical argument to relate counting statistics to the areal density of the IOI in the sensitive volume. Following this, the relationship between statistical uncertainties in the effective attenuation of resonant photons and the uncertainty of the areal density will be derived with an example of estimating the expected precision with which ${ }^{239} \mathrm{Pu}$ content may be measured using a bremsstrahlung spectrum. Next, although the NRF response of ${ }^{240} \mathrm{Pu}$ has not been measured, known NRF resonances of ${ }^{238} \mathrm{U}$ are used to estimate the precision with which a transmission measurement might quantify ${ }^{240} \mathrm{Pu}$ content in spent fuel assemblies. Finally, a generalization between the strength of a resonance and the relative statistical uncertainty a measurement of this resonance would provide is made for low-attenuating assay targets. 


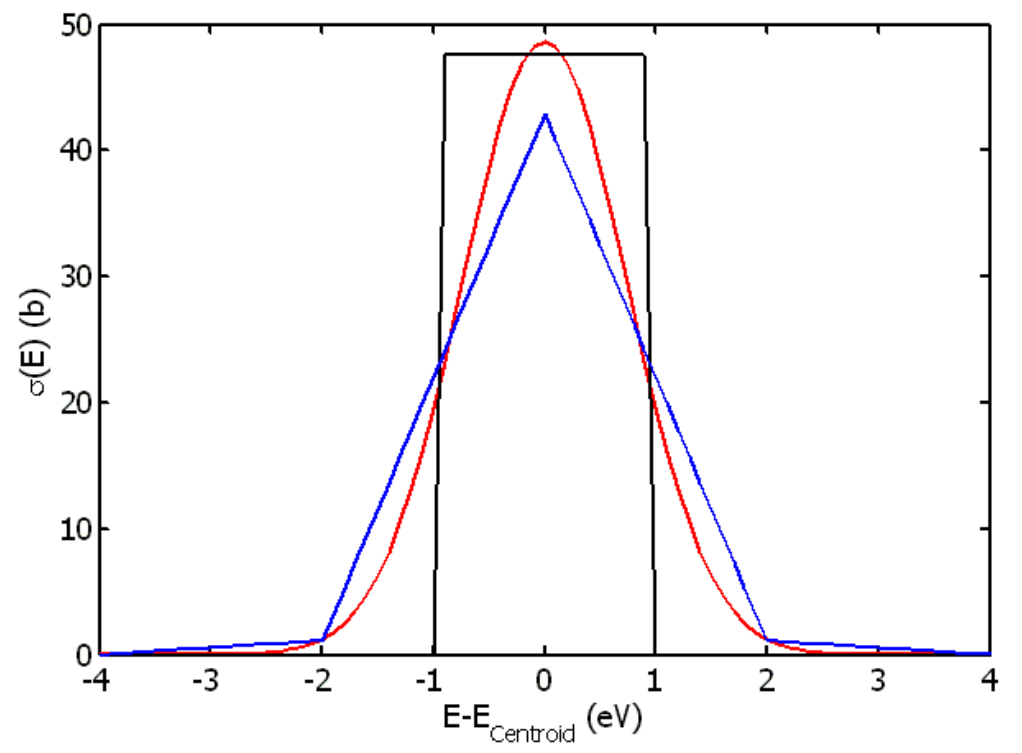

Figure 3.10 Model forms of the resonance cross section profile. Width and total cross section values are from the $2209 \mathrm{keV}$ resonance of ${ }^{238} \mathrm{U}$. Red = Maxwell-Boltzmann, Blue = point-wise, and Black = step function.

\section{Expected Precisions of Pu Areal Density Measurement}

Assume there exists a function for each NRF resonance, $f(N x) \equiv C$ that relates the number of full-energy NRF $\gamma$-ray counts, $C$, to the areal density of the IOI in the target, $N x . N x$ is then given by,

$$
N x=f^{-1}(C)
$$

Likewise, uncertainty in $N x$ can be written as

$$
\sigma_{N x}=\left|\frac{d f^{-1}}{d C}\right| \sigma_{C}
$$

Neglecting down-scatter of photons while they penetrate the assay target and the TD, the expected number of $\gamma$-ray counts, $C$, due to NRF in the TD may be expressed as

$$
C \equiv f(N x)=c_{t} \Phi_{0} \exp \left(-\mu_{\text {atom }} x\right) \mathbb{A}(N x) N_{\mathrm{TD}} \sigma_{\mathrm{NRF}} \frac{P_{f} \epsilon \Delta \Omega}{r_{\text {det }}^{2}}
$$

where $c_{t}$ is the duration of the measurement, and all remaining terms have been defined previously. Only $\mathbb{A}(N x)$ in equation 3.22 is dependent upon $N$, the density of the IOI in the assay target. We re-express $f(N x)$ as,

$$
f(N x)=\chi \mathbb{A}(N x)
$$

where

$$
\chi \equiv c_{t} \Phi_{0} \exp \left(-\mu_{\text {atom }} x\right) N_{\mathrm{TD}} \sigma_{\mathrm{NRF}} \frac{P_{f} \epsilon \Delta \Omega}{r_{d e t}^{2}}
$$

$\chi$ represents the number of NRF $\gamma$-ray counts that are expected to be measured from the TD in the absence of IOI atoms in the assay target. 


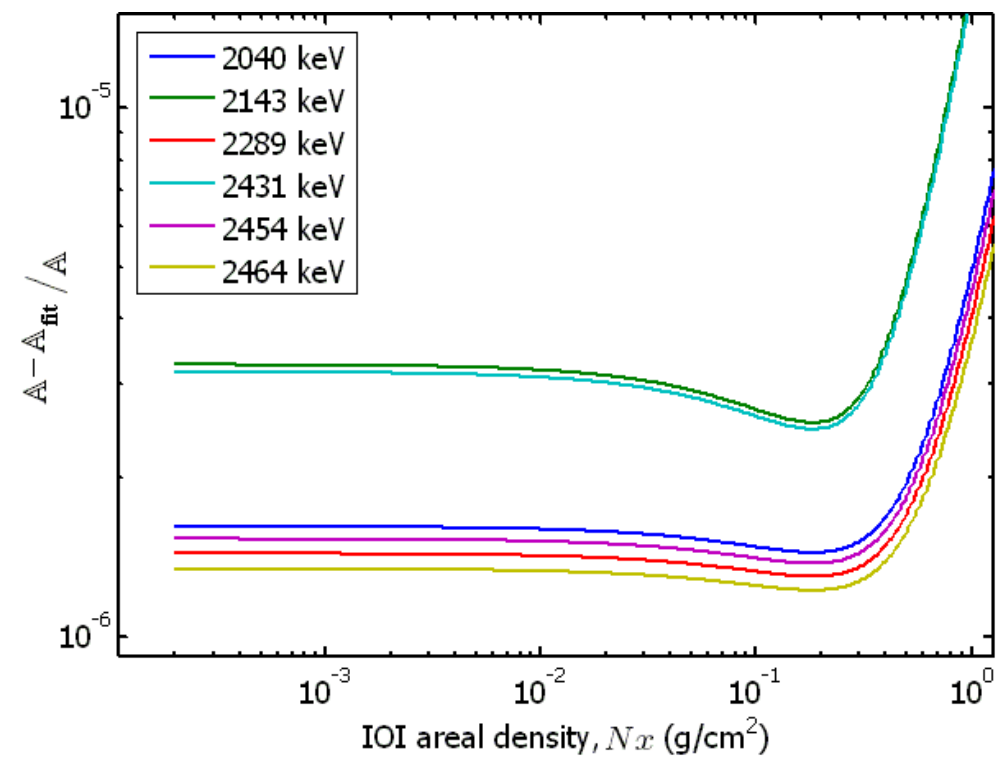

Figure 3.11 Fraction deviation between calculated values of $\mathbb{A}(N x)$ and those resulting from the best-fit of the form shown in equation 3.25 .

The function, $f(N x)$ is therefore directly proportional to $\mathbb{A}(N x)$, the effective attenuation. Unfortunately, the functional form of $\mathbb{A}(N x)$ is somewhat complex, and it cannot be analytically differentiated.

Figure 3.3 shows $\mathbb{A}(N x)$ for ${ }^{239} \mathrm{Pu}$ resonances and a TD thickness of $8 \mathrm{~g} / \mathrm{cm}^{2}$ of ${ }^{239} \mathrm{Pu}$. Stronger resonances result in functions of $\mathbb{A}(N x)$ with a steeper slope at lower areal densities and then flatten for higher values of $N x$.

To obtain an analytically differentiable function that approximates $f^{-1}(C)$, We consider a fit to $\mathbb{A}(N x)$ of the form

$$
\mathbb{A}(N x)_{\text {fit }}=\exp \left(-\alpha_{f} N x\right)
$$

for the range $N x \leq 0.5 \mathrm{~g} / \mathrm{cm}^{2}$, which is approximately the range of areal densities expected for spent fuel assemblies ${ }^{1}$. Comparisons between fits of this form and $\mathbb{A}(N x)$ are shown for ${ }^{239} \mathrm{Pu}$ resonances in Figure 3.11. The fit is quite good over the fitted range, but becomes quite poor for higher $N x$. Best-fit values of $\alpha_{f}$ for each ${ }^{239} \mathrm{Pu}$ resonance are shown in Table 3.3 along with measured resonance parameters from reference[26].

Considering only the cases where $N x \leq 0.5 \mathrm{~g} / \mathrm{cm}^{2}$ and using

$$
f(C) \approx \chi \exp (-\alpha N x)
$$

we can write

$$
f^{-1}(C) \approx \frac{\ln \chi-\ln C}{\alpha}
$$

and

$$
\frac{d f^{-1}}{d C}=\frac{-1}{C \alpha}
$$

\footnotetext{
${ }^{1}$ A fit of the form $\mathbb{A}(N x)_{\text {fit }}=a_{1} \exp \left(a_{2} N x\right)+a_{3} \exp \left(a_{4} N x\right)$ provides quite good agreement over a much larger range of areal densities $\left(\leq 2 \times 10^{-6}\right.$ error fraction for $\left.N x \leq 30 \mathrm{~g} / \mathrm{cm}^{2}\right)$, but it also does not provide an analytical form for $d f^{-1}(C) / d C$.
} 
Using equation 2.23, which relates the statistical precision of a $\gamma$ ray counting measurement to the ratio of signal-to-background intensities, $\xi$, and substituting equation 3.28 into equation 3.21, we have

$$
\sigma_{N x}=\frac{\sqrt{1+2 / \xi}}{\alpha \sqrt{C}}
$$

This relates the precision with which $N x$ is measured to both counting statistics and the quantity $\alpha$, which is the magnitude of the slope of the effective attenuation curve at the corresponding areal density.

From equation 3.29, we can infer that large resonances will provide the smallest statistical errors when the IOI areal density in the target is small, as is the case for measuring $\mathrm{Pu}$ in spent fuel assemblies. The results of these models are applied to the evaluation of measurement methods using bremsstrahlung and quasi-monoenergetic photon sources in Sections 4.2 and 5.2, repectively.

\section{Resonance Strength for Transmission Measurements}

To illustrate the importance of the resonance strength for providing precise measurements of target areal densities, we consider a case where $N x$ is still assumed to be small (i.e., where equation 3.25 remains valid), and compare two hypothetical resonances, $a$ and $b$. Assume these resonances have widths, $\Gamma_{a}$ and $\Gamma_{b}$, respectively, and that $\Gamma_{a}>\Gamma_{b}$. The relative uncertainties in areal density that transmission measurements made using resonance $a$ will be reduced by at least

$$
\frac{\sigma_{a}}{\sigma_{b}} \approx\left(\frac{\Gamma_{b}}{\Gamma_{a}}\right)^{1.5-2}
$$

relative to an identical measurement using resonance $b$. This is the combination of three factors. First, stronger resonances result in proportionally higher NRF count rates, which, reduces the

uncertainty by a factor of $\left(\frac{\Gamma_{b}}{\Gamma_{a}}\right)^{1 / 2}$. Also, stronger resonances increase the slope of the effective attenuation function, $\alpha$, which result in a relative decrease in uncertainty of approximately $\left(\frac{\Gamma_{b}}{\Gamma_{a}}\right)$. Finally, for NRF signals not significantly stronger than background intensity, the term $\sqrt{1+2 / \xi}$ in equation 3.29 can contribute an additional factor of up to $\left(\frac{\Gamma_{b}}{\Gamma_{a}}\right)^{1 / 2}$.

\subsection{MCNPX Modeling}

We have used the MCNPX code extensively to study the use of NRF for measuring Pu content in spent fuel. MCNPX was used to generate bremsstrahlung spectra, simulate photon transport in both the backscatter and transmission geometries, test the analytical models derived to compute NRF fluences in Sections 3.1 and 3.2, and to test the effect of notch refill in Section 3.3.4.

It became evident in our initial study of NRF for spent fuel assay that MCNPX needed additional capabilities to become a useful tool for modeling NRF responses and background. Critical to this effort, we have added existing NRF data to the MCNPX photonuclear data files and are now able to include NRF physics into MCNPX simulations. During the modeling studies, it was also recognized that MCNPX does not accurately calculate photon elastic scatter that is a significant contributor to the background for measurement of NRF signals. This was remedied by modification of the photon transport routines used by MCNPX and by reconstructing the photo-atomic 
Table 3.2 Characteristics of measured NRF states in ${ }^{235} \mathrm{U}$ as reported in reference[26]. Values of $g \Gamma_{0}$ were obtained by application of equation 2.2. States where $\Gamma_{0} / \Gamma \neq 1$ indicate that a $\gamma$ ray of energy, $E_{\gamma}=E_{C}-46.2$ $\mathrm{keV}$ was observed. These lines are assumed to correspond to de-excitation of the NRF state via emission of a $\gamma$ ray populating the low-lying $9 / 2^{-}$excited state of ${ }^{235} \mathrm{U}$. Values of $\int \sigma_{\mathrm{NRF}} d E$ shown here are the sum of the reported values.

\begin{tabular}{|c|c|c|c|c|c|c|c|}
\hline \multicolumn{2}{|c|}{$\begin{array}{c}E_{\text {level }} \\
(\mathrm{keV})\end{array}$} & \multicolumn{2}{|c|}{$\begin{array}{c}\mathrm{g} \Gamma \\
(\mathrm{meV})\end{array}$} & \multicolumn{2}{|c|}{$\begin{array}{c}\mathrm{g} \Gamma_{0} \\
(\mathrm{meV})\end{array}$} & \multicolumn{2}{|c|}{$\begin{array}{l}\sigma_{\mathrm{NRF}} d E \\
(\mathrm{eV} \cdot \mathrm{b})\end{array}$} \\
\hline 56.23 & .80 & 1.47 & \pm 0 . & 1.47 & \pm 0 & 4.1 & \pm 1 \\
\hline & & 17 & \pm & & & 9 & \pm \\
\hline &.. & 8.79 & \pm & & & 14 & \pm 1 \\
\hline & $=.2$ & 2.9 & & & & & \pm 1. \\
\hline & $=.2$ & 4.3 & & & & . 6 & \pm 1. \\
\hline & & & & & & 9.7 & \pm 1 \\
\hline 2006.19 & .01 & 2.40 & 10.04 & 2.40 & \pm 0.84 & 4.7 & $I 1$ \\
\hline
\end{tabular}

datafiles used by MCNPX to include extended descriptions of the form factors used to describe the elastic photon scatter process. This is described in more detail in Appendix D.

\subsubsection{Addition of Available NRF Resonance Data to ENDF and MCNPX Datafiles}

In order to be able to model the NRF response in MCNPX measured NRF data were added to the photonuclear evaluated nuclear data files (ENDF) and were converted to ACE files for use in MCNPX [29]. To maintain compatibility with existing data processing codes such as NJOY [30], cross sections for NRF interactions have been added as pointwise data and characteristic NRF $\gamma$ rays are added as secondary particles emitted by the reaction. The energy width between consecutive data points was selected to be a minimum of $2 \mathrm{eV}$ due to ENDF format limitations. Most NRF resonances have natural linewidths on the order of millieV (meV). To describe NRF resonances using such a coarse energy scale, the resonances were Doppler broadened assuming a temperature, $T$, of $300 \mathrm{~K}$. The resulting resonance form is due to Maxwell-Boltzmann statistics:

$$
\sigma_{D}(E)=\int \sigma(E) d E \frac{1}{E_{0}} \sqrt{\frac{m c^{2}}{\pi k T}} \exp \left(\frac{-\left(E-E_{0}\right)^{2}}{E_{0}^{2} k T / m c^{2}}\right)
$$

This form more closely resembles the shape of NRF resonances experienced by incident photons because thermal motion of atoms within materials causes the photons to experience a Dopplershifted resonance. Similar to the implementation of NRF cross sections, the energies of emitted NRF $\gamma$ rays are entered into ENDF pointwise, and the multiplicity for photon emission is defined for each resonance.

The NRF responses of ${ }^{235} \mathrm{U}$ and ${ }^{239} \mathrm{Pu}$ were measured in 2006 and 2007 [26], and the results from these experiments are presented in Tables 3.2 and 3.3, respsectively. Several pairs of observed NRF $\gamma$ rays were found to have energy differences equal to the excitation energy of low-lying excited states $\left(7.9 \pm 0.002 \mathrm{keV}\right.$ for ${ }^{239} \mathrm{Pu}$ and $46.21 \pm 0.01 \mathrm{keV}$ for $\left.{ }^{235} \mathrm{U}\right)[31]$. In these cases the sum of the measured cross sections is shown in the table, and the tabulated values of $\Gamma_{0}$ and $\Gamma$ differ. Subsequent experiments attempting to measure higher-energy NRF resonances in these isotopes have not yielded new transitions[32, 33]. 
Table $3.3{ }^{239} \mathrm{Pu}$ NRF data. The values of $\int \sigma_{\mathrm{NRF}} d E$ were taken from Reference [26]. Where $g \Gamma_{0} \neq g \Gamma$, two NRF $\gamma$ rays were measured at energies that differ by the excitation energy of the first excited state of ${ }^{239} \mathrm{Pu}$. It has been assumed that these $\gamma$ rays correspond to de-excitation of a single NRF state. Values of $\mathrm{g} \Gamma_{0}$ were calculated from equation 2.2. The meaning of $\alpha_{f}$ is explained in the text.

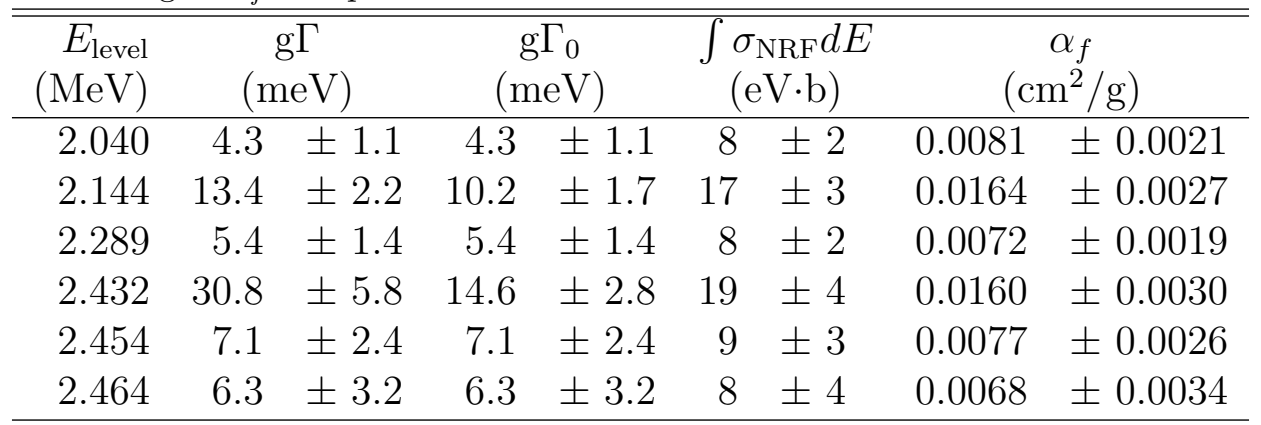

Table 3.4 Relevant ${ }^{238} \mathrm{U}$ NRF data. Values of $\Gamma_{0}^{2} / \Gamma$ and $\Gamma_{1} / \Gamma_{0}$ were taken from reference[27] where $\gamma$-ray emission to the first excited state of ${ }^{238} \mathrm{U}\left(J^{\pi}=2^{+}\right)$at $44.92 \mathrm{keV}$ were also measured. Here, it is assumed that this probability is $1-\Gamma_{0} / \Gamma$. The final column is calculated from equation 2.2 , it has a fractional uncertainty equal to that of $\Gamma_{0}$.

\begin{tabular}{rrrrrrr}
\hline \hline $\begin{array}{r}E_{\text {level }} \\
(\mathrm{MeV})\end{array}$ & \multicolumn{2}{c}{$\Gamma$} & \multicolumn{2}{c}{$\begin{array}{c}\Gamma_{0} \\
(\mathrm{meV})\end{array}$} & \multicolumn{2}{c}{$\begin{array}{c}\int \sigma_{\mathrm{NRF}} d E \\
(\mathrm{meV})\end{array}$} \\
\hline 2.176 & 54.7 & \pm 2.5 & 36.0 & \pm 2.2 & 87.7 & \pm 5.2 \\
2.209 & 54.3 & \pm 2.8 & 35.0 & \pm 2.3 & 82.7 & \pm 5.1 \\
2.245 & 29.0 & \pm 1.6 & 19.7 & \pm 1.4 & 45.0 & \pm 3.0 \\
2.295 & 13.4 & \pm 1.5 & 8.4 & \pm 1.0 & 14.4 & \pm 1.4 \\
2.410 & 26.0 & \pm 1.7 & 16.9 & \pm 1.2 & 33.6 & \pm 2.1 \\
2.468 & 30.2 & \pm 2.2 & 20.1 & \pm 1.6 & 38.0 & \pm 2.8 \\
\hline
\end{tabular}




\subsubsection{MCNPX Simulation of Backscatter Measurements with Bremsstrahlung}

With the addition of NRF data to MCNPX datafiles and the inclusion of elastic photon backscatter to MCNPX, the code may be directly used to determine expected photon intensities and count rates that would be expected in backscatter NRF measurements. The results of these measurements with bremsstrahlung photon sources are described in Section 4.1. Here, the calculation process is described.

The primary difficulty in simulating backscattered NRF is that the vast majority of photons that interact within the assay target will produce low-energy or forward-scattered photons. However, the energetic portion of the backscattered photon spectrum is of most interest. To overcome these difficulties, many variance reduction techniques are used.

First, simulation of electrons is time-consuming. Therefore, the simulation of bremsstrahlung source spectra is done separately. Thereafter, the calculated bremsstrahlung spectrum is used as a source term for separate simulations.

A second simulation is conducted with the bremsstrahlung photons incident upon a target in the backscatter NRF assay geometry. Sampling of the bremsstrahlung photon source spectrum is biased to preferentially simulate photons of higher energies, with reduced statistical weights. The photon flux at a hypothetical detector location is then calculated using the next-event estimator tally (F5) wherein each time a particle interacts in the assay target, the probability for emission of a particle in the direction of the detector is determined, and a value proportional to this quantity is added to the flux tally. The spectra shown in Figure 4.1 were determined by such a calculation.

In a third simulation, the response of a radiation detector is simulated by impinging the photon spectrum determined in the second calculation onto a shielded detector geometry, such as that described in Section F.4.1. The energy deposited within the detector volume is tallied, and if necessary, an energy resolution-broadening routine is applied. These simulations provide the total and NRF count rates that are expected for a given assay geometry.

\subsubsection{MCNPX Simulation of Resonant Attenuation}

In addition to determining expected NRF and background signal rates for transmission measurements using bremsstrahlung sources (as described in Section 3.2.1), MCNPX was also used to test the effective attenuation model. This is done by determining the NRF flux at a detector position when no resonant absorption occurred in the assay target and comparing that to the NRF flux when the $\mathrm{Pu}$ IOI is present in the target.

NRF peak intensities are calculated using MCNPX for two geometries, one in which the $\mathrm{Pu}$ IOI is present in the fuel assembly, and another in which it is absent. The removal of the IOI from the assembly results in slight changes in the non-resonant attenuation of photons as they penetrate the assembly. To correct for this non-resonant attenuation change, the intensity of NRF peaks is normalized by the intensity of the $511 \mathrm{keV}$ peak which is entirely due to pair production in the TD, i.e.,

$$
\mathbb{A}_{E_{p k}}=\frac{A_{E_{p k}}}{A_{E_{p k}}^{n o P u}} \frac{A_{511}^{n o P u}}{A_{511}}
$$

This method is described and examined in Section A.5, wherein experimental data agreed within statistics to that analytically predicted. Simulations also indicate that systematic uncertainties associated with this normalization routine are likely to be near $1 \%$ when two targets that differ in 


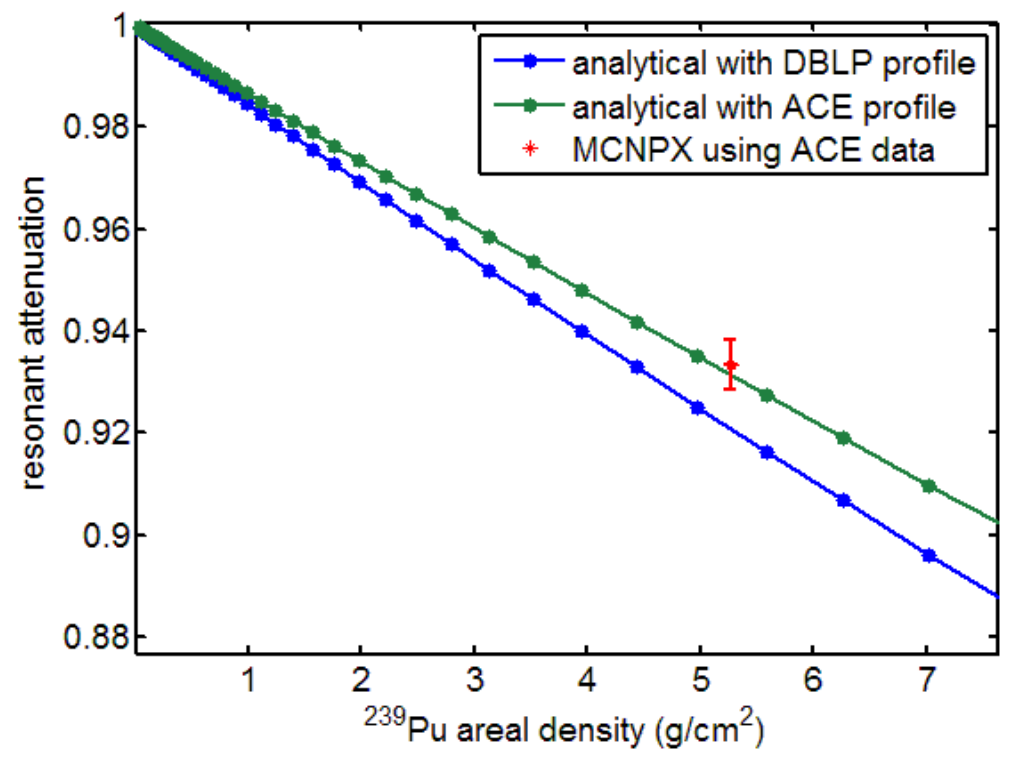

Figure 3.12 Comparison of analytically-calculated effective attenuation values with that calculated by MCNPX. The different analytical curves correspond to the expected true form (blue) and that due to the shape of the NRF data entered into the ACE file used by MCNPX.

actinide content by $9.6 \mathrm{wt} . \%$ are compared. Although more detailed examination of this method would be advisable, it shows promise to correct for beam normalization and fuel assembly geometry differences.

The quantity $\mathbb{A}_{E_{p k}}$, is used in equation 3.19 for the corresponding resonance to determine the expected areal density of $\mathrm{Pu}$ in the target. Given that the $\mathrm{Pu}$ areal density is controlled and known in MCNPX simulations, these simulations serve as computational checks on the analytical model.

Figure 3.12 presents comparisons between analytical effective attenuation values (described in Section 3.2.2) and those obtained using the analysis method described here to evaluate $\mathbb{A}_{E_{p k}}$. Two analytical curves are provided. The blue curve is the analytically-expected attenuation due to the $2431 \mathrm{keV}{ }^{239} \mathrm{Pu}$ resonance, as in equation 3.19. The green curve is the expected attenuation due to the $2431 \mathrm{keV}{ }^{239} \mathrm{Pu}$ resonance if the 5 point evaluation of the Maxwell-Boltzmann cross section is used as the cross section form (as described in Section 3.2.4). This curve is shown because the 5 point evaluation profile is used to described resonances used in MCNPX (in the ACE data format). The red point with error bars indicates an effective attenuation value obtained via MCNPX calculations. Although the MCNPX value overlaps the green curve within the error bars, it is anticipated that the true value would lay above the curve, due to the effects of notch refill.

\subsubsection{Notch Refilling and Transmission Assay Complications}

In transmission measurements, the attenuation of resonant-energy photons in the target provides the signal that relates to areal density of the IOI in the target. This attenuation manifests itself in a decrease in the flux intensity at the resonance, which is often referred to as a notch. Notch refilling is the process by which interrogating photons are down-scattered into the notch during transport through the assay target and TD. The down-scatter of photons results in more resonant-energy 


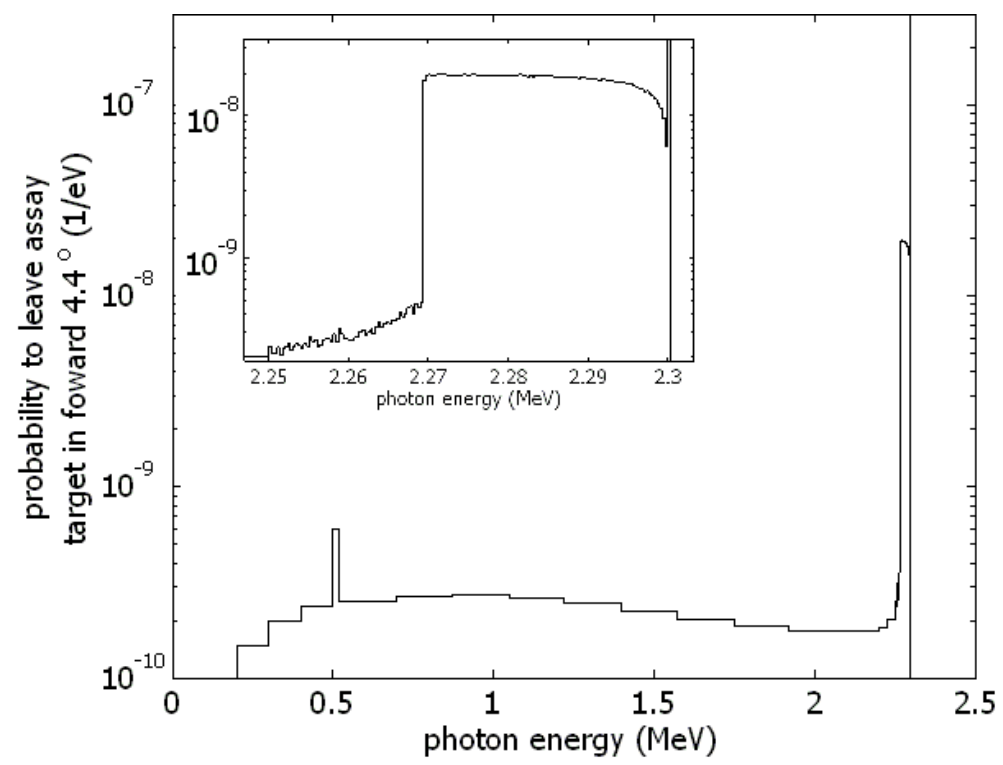

Figure 3.13 Calculated spectrum of photons leaving a target of areal density similar to a homogenized fuel assembly within $4.4^{\circ}$ of the initial photon direction. The incident photon energy was $2.3 \mathrm{MeV}$. The inset shows a larger view of the energy range of $2.25-2.3 \mathrm{MeV}$. The probability that an incident photon leaves the target without interacting is $2.1 \%$.

photons leaving the assay target than would be predicted by the model described in Section 3.2.2. In this section, the relative intensity of the notch-refilling effect is considered.

Notch refilling depends not only on the shape of the energy spectrum, but also on the measurement geometry because the down-scattered photons must interact in the TD in order to change the measured signal. The smaller the solid angle subtended by the TD, the more restricted is the geometry for notch refilling. Likewise, increasing the number of photons that have more energy than the excitation energy of a resonance increases the likelihood that some of these photons will down-scatter into the notch created by resonant absorption.

The processes that result in notch refilling are incoherent scattering and bremsstrahlung emitted from photo-electrons. Both processes predominantly produce down-scattered photons predominantly in forward angles. Those photons that are down-scattered into a resonant energy and toward the TD effectively refill the notch.

To examine this effect, we first consider a MCNPX simulation of $2.3 \mathrm{MeV}$ photons incident upon an assay target. The target consisted of a $7 \mathrm{~cm}$ thick slab of $\mathrm{Pb}$ behind a $4.4 \mathrm{~mm}$ thick slab of DU, which is comparable to the average areal density of a fuel assembly. The energy spectrum of photons leaving the assay target within $4.4^{\circ}$ of the initial direction were calculated. The selection of $4.4^{\circ}$ is representative of the solid angle that would be subtended by a large TD. The results of this simulation are shown in Figure 3.13. Full-energy photons and those that have undergone a single incoherent scatter are the most probable sources of emitted photons. The photons that undergo a single incoherent scatter produce a continuum of photons between 2.27 and $2.3 \mathrm{MeV}$. Pair production and subsequent positron annihilation, multiple-scattering events, and secondary electron bremsstrahlung produce the events indicated below the Compton continuum.

A $2.3 \mathrm{MeV}$ initial energy photon undergoing incoherent scattering with $\theta \leq 4.4^{\circ}$ results in a scattered photon with energy $E^{\prime} \geq 2.27 \mathrm{MeV}$. This energy is the lower energy of the Compton continuum. The probability per $2.3 \mathrm{MeV}$ incident photon for an emitted photon to be down- 
scattered into the Compton continuum is approximately $2 \times 10^{-8}$ per eV. Doppler-broadened NRF resonances tend to have $\Gamma \approx 1.5 \mathrm{eV}$, thus we can assume $3 \times 10^{-8}$ per eV is a reasonable probability for down-scattering of $2.3 \mathrm{MeV}$ photons into its Compton continuum for assay targets of similar areal densities as fuel assemblies.

To consider the potential for refill of a given resonant notch, only photons incident upon the assay target whose Compton continuum includes the resonance energy are of importance. If the TD only subtends a polar angle of $4.4^{\circ}$ only photons with $E-E_{\text {res }} \lesssim 35 \mathrm{keV}$ need be considered. From Figure 2.6, we expect $\sim 2 \times 10^{8}$ photons/eV/Coulomb to impinge upon the assay target in the energy range of $2-2.4 \mathrm{MeV}$. Combining this source photon rate with the notch-refill probability $\left(\sim 2 \times 10^{-8}\right.$ per $\left.\mathrm{eV}\right)$ and the range of energies of interest, we expect $\sim 1.4 \times 10^{5}$ notch refill events per Coulomb of electrons incident upon the bremsstrahlung converter. Conversely, if an entire bremsstrahlung spectrum were transmitted through this target, we would expect in the absence of resonant attenuation, $\sim 4.5 \times 10^{6}$ photons $/ \mathrm{eV} / \mathrm{C}$ to be incident upon the TD between 2 and 2.4 $\mathrm{MeV}$. Thus, photons that arrive at the TD, after down-scattering, represent $2-5 \%$ of the total flux in this energy range.

From these estimates, we expect that notch refilling will result in a slight decrease in the slopes of the effective attenuation curves, $\mathbb{A}(N x)$ (Figure 3.3), compared to those predicted by neglecting notch refill. This, in turn, would slightly increase the statistical uncertainties estimated in Table 4.3 and the surrounding discussion. Nevertheless, notch refilling would change the statistical uncertainty estimates for a measurement of a $\mathrm{Pu}$ isotope in spent fuel significantly less than the contributions from uncertainties in the measured NRF cross sections. We therefore conclude that while notch refilling is likely to be an important factor in achieving an absolute areal density measurement with precision near 1\%, the NRF cross sections must be known to far better precision before this level of uncertainty can be reached.

\section{Division of Simulations Into Resonant and Non-resonant Problems}

The simulation of NRF measurements that use quasi-monoenergetic photon sources can be treated differently than bremsstrahlung source simulations because of the narrow range of photon energies that must be considered. This implies that non-resonant source photons will behave similarly, regardless of their initial energy. To take advantage of this, two separate simulations are conducted for assay using quasi-monoenergetic photons, one with source photons of resonant energy, and the other where the effects of NRF is turned off, which only simulates the transport of non-resonant photons.

These two simulations may be combined by summing the two spectra, weighted by the relative number of resonant and non-resonant photons in the source spectrum. Since the NRF resonances were entered into the ACE data files as 5 data points each separated by $2 \mathrm{eV}$, the total width of the resonance, described in MCNPX, is $8 \mathrm{eV}$ although the FWHM is much closer to $2 \mathrm{eV}$ (see Figure 3.10). Because of this, the resonant-energy portion of the source photon spectrum is frequently considered to be $8 \mathrm{eV}$ wide, centered on the nominal resonance centroid energy. This decomposition method is used throughout Section 5. 


\section{Determining $\mathrm{Pu}$ in Spent Fuel Using Available Technology}

We have studied and evaluated the potential of NRF-based assay methods that employ currently available bremsstrahlung sources and $\gamma$-ray detectors. In this section the backscatter and transmission methods are discussed with examples of modeled measurements.

Nuclear resonance fluorescence produces isotope-specific signatures. Therefore the prospect of using NRF to measure $\mathrm{Pu}$ content in spent fuel must be separately considered for each $\mathrm{Pu}$ isotope. The only $\mathrm{Pu}$ isotope for which the $\mathrm{NRF}$ response has been measured is ${ }^{239} \mathrm{Pu}$. However, nuclides with even numbers of protons and neutrons tend to have stronger NRF resonances than odd-nucleon isotopes. We therefore consider the NRF response of ${ }^{238} \mathrm{U}$ as relevant to predicting the response from ${ }^{240} \mathrm{Pu}$.

In this section the use of models and MCNPX simulations to predict expected nuclear resonance fluorescence detection rates for non-destructive materials assay studies is presented along with predictions of background rates. These models assume that NRF is detected by a single radiation detector, and that use of multiple detectors would proportionally increase the calculated count rates.

\subsection{Backscatter Measurement of Spent Fuel Pins}

To ascertain the likelihood that NRF could be used to assay spent fuel, the backscatter measurement technique was examined. The modeled geometry for the assay is similar to that shown in Figure 2.3, except that the fuel assembly indicated in the drawing is replaced by a cylindrical fuel pin. The pin was assumed to be a $10 \mathrm{~mm}$ diameter $\mathrm{UO}_{2}$ cylinder.

$\gamma$-ray interactions counted by radiation detectors that are not due to NRF are considered background. These events may be due to scatter of the interrogating photon beam or radioactive decay of material in the assay geometry. It is also important to differentiate between background events at and near resonant energies and those at other energies. The intensity of radioactive emission is estimated in Appendix B and the results are used here.

\section{Backscattered Background}

The physical processes that result in photons being emitted at backwards angles relative to the incident photon direction are incoherent scatter, coherent scatter, pair production, and bremsstrahlung emission from photoelectrons. Incoherent scatter produces only low-energy backscattered photons and pair production results in $511 \mathrm{keV}$ photons in all directions. Coherent scattering on the other hand produces energetic photons, and while the coherent scattering processes are predominantly forward-directed, but do also result in some energetic backscattered radiation, which necessitated modification of the MCNPX coherent scatter routine. Similarly, multi-step processes such as multiple incoherent scattering events or bremsstrahlung emission from energetic photoelectrons can result in energetic backscattered radiation. 


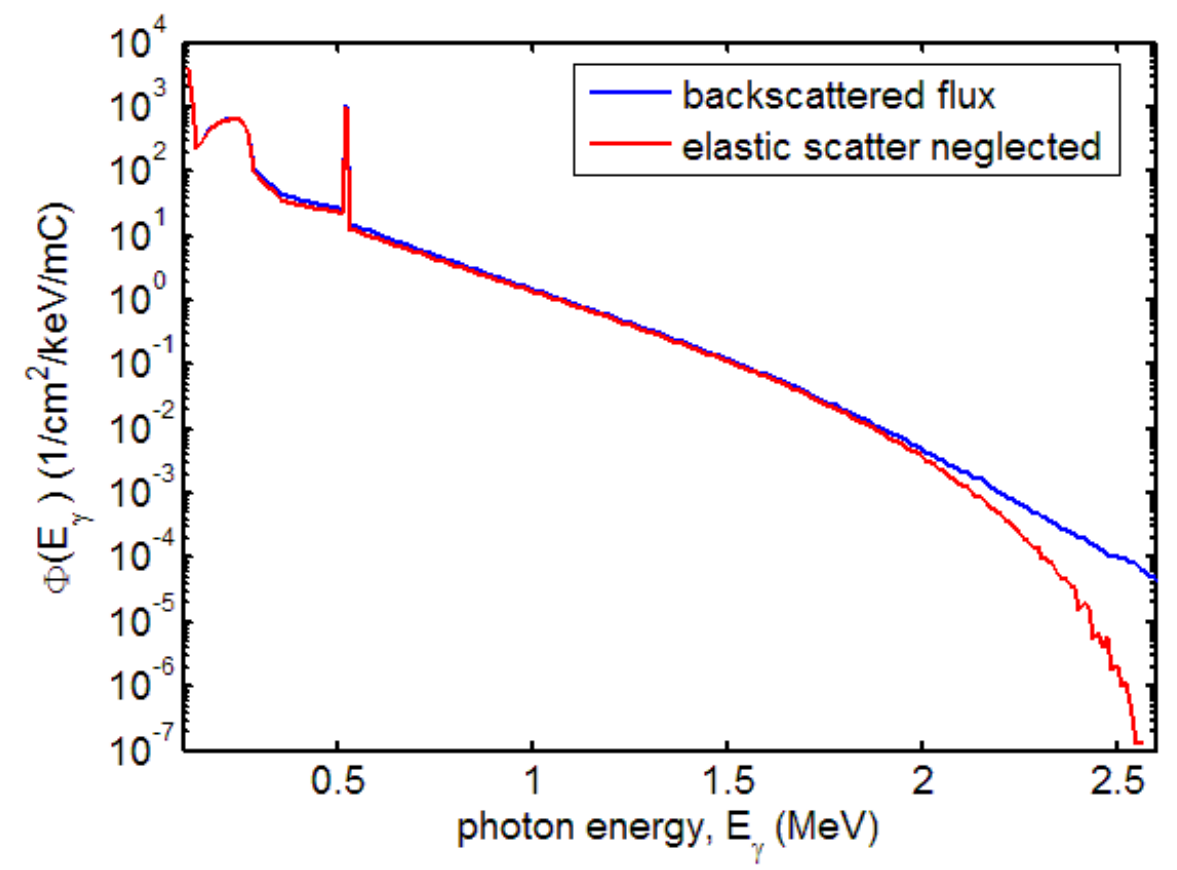

Figure 4.1 Calculated backscattered photon flux for assay of a $5 \mathrm{~mm}$ diameter cylinder of spent BWR elements at a detection position $100 \mathrm{~cm}$ from the target, at $135^{\circ}$ relative to initial electron beam direction. The flux is normalized per $\mathrm{mC}$ of $2.6 \mathrm{MeV}$ electrons incident upon bremsstrahlung converter. The red curve indicates the photon flux calculated by MCNPX before the elastic photon scatter routines were corrected.

The background for the case studied here was simulated in MCNPX. The interrogating radiation was generated with $2.6 \mathrm{MeV}$ electrons incident upon a $102 \mu \mathrm{m}$ Au foil on a $1 \mathrm{~cm}$ thick $\mathrm{Cu}$ backing. This simulation produced the spectrum shown in Figure 2.6. The resulting bremsstrahlung was collimated to $1^{\circ}$ half-angle and used to irradiate the $\mathrm{UO}_{2}$ cylinder $30 \mathrm{~cm}$ downstream. The resulting backscattered fluence, shown in Figure 4.1, was calculated $100 \mathrm{~cm}$ from the target in a viewing window located at an angle of $135^{\circ}$ relative to the direction of the electron beam.

The energy-integrated fluence for the spectrum shown in Figure 4.1 is approximately $1.6 \times 10^{5}$ photons $/ \mathrm{cm}^{2} / \mathrm{mC}$. With the front surface area of a large HPGe detector of $\sim 50 \mathrm{~cm}^{2}$, the detector count rate would be too high with a bremsstrahlung source of reasonable intensity and therefore thick filters (e.g. $7.5 \mathrm{~mm}$ of $\mathrm{Pb}$ ) must be used to reduce count rates due to low-energy photons emitted and scattered from the spent fuel.

The expected background photon spectrum due to detection of non-resonantly scattered interrogation photons is determined by simulating the shielded detector response to the calculated photon flux shown in Figure 4.1. The resulting values at resonant energies are tabulated as $R_{\mathrm{bkg}}$ in Table 4.1. The total intensity is given by integrating the energy-differentiated spectrum over all energies. This results in $4.6 \times 10^{2}$ counts $/ \mathrm{mC}$. Using a $45 \mathrm{~mA}$ electron beam to produce the bremsstrahlung beam would then result in approximately $10^{4}$ background counts/s in the counting geometry. Adding the simulated counting rate due to the radioactivity of the spent fuel of $10^{4}$ counts/s (See Appendix B), yields a total count rate of $\sim 2 \times 10^{4}$ counts/s. 


\section{NRF Count Rates from Backscatter Assay of Spent Fuel Pin}

Equation C.3 was evaluated for the example assay geometry where ${ }^{239} \mathrm{Pu}$ comprises $0.4 \%$ of the actinide mass. The interrogating beam was a $2.6 \mathrm{MeV}$ endpoint-energy bremsstrahlung beam. The detector response was modeled as described in Section F.4 and due to spent fuel radioactivity, was assumed to be shielded in the configuration shown in Figure F.4, with a filter thickness, $x_{f}=7.5$ $\mathrm{cm}$.

The resulting expected count rates of $\mathrm{NRF} \gamma$ rays from ${ }^{239} \mathrm{Pu}$ are tabulated in Table 4.1. The values are reported in units of counts $/ \mathrm{mC}$, indicating that rates are proportional to the intensity of the electron beam that generates the bremsstrahlung beam. For sources of background that are continuous in energy, the energy-differentiated photon flux is multiplied by the detector's energy resolution, which is assumed to be $3 \mathrm{keV}$. The expected uncertainties in the rate at which NRF $\gamma$ rays would be counted are primarily due to experimental uncertainties in the measured strengths of the NRF resonances. These values are up to $33 \%$ for the smaller resonances. Likewise, effects due to detector dead-time will proportionally decrease all rates listed in Table 4.1.

Referring to the results shown in Table 4.1, we observe that a beam current of $\sim 200 \mathrm{~mA}$ would be necessary for the intensity of NRF $\gamma$ rays from the $2143 \mathrm{keV}{ }^{239} \mathrm{Pu}$ resonance to be equal to that due to the radioactivity of spent fuel, 11 years after reactor discharge. With this beam intensity in mind, we now consider the intensity of non-resonantly backscattered photons, which also scales with the intensity of the interrogating beam.

The significantly larger intensity of the non-resonantly scattered photons and the radioactive decay photons, relative to the NRF $\gamma$ rays causes the measurement of backscattered NRF $\gamma$ rays needed to determine spent fuel content to be difficult. Since longer cooling times can reduce the photon intensity due to radioactive decay in spent fuel, the fundamental limit is defined by the fact that the intensity of NRF $\gamma$ rays, relative to those non-resonantly scattered, is fixed and small. This indicates that very large numbers of photon counts would be required to precisely measure the quantity of ${ }^{239} \mathrm{Pu}$ in spent fuel and that information from multiple resonances should be combined, if possible.

\section{Combining Data from Multiple Resonances}

To estimate the total uncertainty of the areal density of ${ }^{239} \mathrm{Pu}$, we assume that the isotopic $\mathrm{Pu}$ concentration value is determined by taking a weighted mean of the values expected from measurement of the individual NRF lines.

The areal density determined by measurement of each NRF line, $\rho_{i}$, is given by

$$
\rho_{i}=\frac{N_{i}}{t I_{e} \epsilon_{i}}
$$

where $t$ is the duration of the measurement, $I_{e}$ is the bremsstrahlung-inducing beam current, $\epsilon_{i}$ is a proportionality constant that relates the number of NRF counts per coulomb of bremsstrahlunginducing electrons to the $\mathrm{Pu}$ isotope concentration, and $N_{i}$ is the number of counts $\left(N_{i}=R_{\mathrm{NRF}} I_{e} t\right)$.

To estimate the total uncertainty of the areal density of ${ }^{239} \mathrm{Pu}$, we assume that the areal density value is determined from a weighted mean of the measured areal densities from each resonance. The weighted mean is given by

$$
\rho=\frac{\sum_{i=1}^{n} w_{i} \rho_{i}}{\sum_{i=1}^{n} w_{i}}
$$


Table 4.1 Expected rates at which ${ }^{239} \mathrm{Pu}$ NRF $\gamma$ rays $\left(R_{\mathrm{NRF}}\right)$, non-resonantly backscattered interrogation photons $\left(R_{\mathrm{bkg}}\right)$, and photons emitted via radioactive decay spent fuel that has cooled for 11 years $\left(R_{\text {fuel }}\right)$, are detected at energies corresponding to ${ }^{239} \mathrm{Pu}$ resonance $\gamma$ rays. The detector was assumed to be a $100 \%$ relative efficiency HPGe shielded behind $7.5 \mathrm{~cm}$ lead, with assumed energy resolution of $3 \mathrm{keV}, 100 \mathrm{~cm}$ from the center of the fuel pin at an angle of $135^{\circ}$, relative to the initial electron beam direction. Values in the last row represent total rates expected over the entire spectrum.

\begin{tabular}{ccccc}
\hline \hline $\begin{array}{c}\text { Centroid } \\
\text { Energy }(\mathrm{keV})\end{array}$ & $\begin{array}{c}\int \sigma_{\mathrm{NRF}} d E \\
(\mathrm{~b} \cdot \mathrm{eV})\end{array}$ & $\begin{array}{c}R_{\mathrm{NRF}} \\
(1 / \mathrm{mC})\end{array}$ & $\begin{array}{c}R_{\mathrm{bkg}} \\
(1 / \mathrm{mC})\end{array}$ & $\begin{array}{c}R_{\text {fuel }} \\
(1 / \mathrm{s})\end{array}$ \\
\hline 2040.3 & 8 & $2.5 \times 10^{-5}$ & $4.2 \times 10^{-3}$ & $9 \times 10^{-3}$ \\
2135.0 & 4 & $1.1 \times 10^{-5}$ & $2.4 \times 10^{-3}$ & $8 \times 10^{-3}$ \\
2143.6 & 13 & $3.3 \times 10^{-5}$ & $2.2 \times 10^{-3}$ & $8 \times 10^{-3}$ \\
2289.0 & 8 & $1.6 \times 10^{-5}$ & $6.9 \times 10^{-3}$ & $6 \times 10^{-3}$ \\
2423.5 & 10 & $1.4 \times 10^{-5}$ & $2.3 \times 10^{-4}$ & $5 \times 10^{-3}$ \\
2431.7 & 9 & $1.2 \times 10^{-5}$ & $2.2 \times 10^{-4}$ & $5 \times 10^{-3}$ \\
2454.4 & 9 & $1.1 \times 10^{-5}$ & $1.8 \times 10^{-4}$ & $4 \times 10^{-3}$ \\
2464.6 & 8 & $9.6 \times 10^{-6}$ & $1.6 \times 10^{-4}$ & $3 \times 10^{-3}$ \\
\hline & totals & $1.3 \times 10^{-4}$ & $4.6 \times 10^{2}$ & $1 \times 10^{4}$ \\
\hline
\end{tabular}

where $n$ is the number of NRF $\gamma$ rays considered (eight for Table 4.1) and the weights are

$$
w_{i}=1 / \sigma_{\rho_{i}}^{2}
$$

The standard deviation for the weighted mean is given by

$$
\sigma_{\rho}=\sqrt{\sum_{i=1}^{n}\left(\sigma_{\rho_{i}} \frac{d \rho}{d \rho_{i}}\right)^{2}}
$$

where

$$
\frac{d \rho}{d \rho_{i}}=\frac{1}{\sigma_{\rho_{i}}^{2}} \frac{1}{\sum_{i=1}^{n} w_{i}}
$$

Using equation 4.1 and that $\sigma_{N} / N=\sqrt{1+2 / \xi} / \sqrt{n}$, we obtain

$$
\sigma_{\rho_{i}}=\frac{\sqrt{N_{i}(1+2 / \xi)}}{\epsilon t I_{e}}
$$

The signal, relative to the background intensity is given by

$$
\xi=\frac{I_{e} R_{\mathrm{NRF}}}{I_{e} R_{\mathrm{bkg}}+R_{\text {fuel }}}
$$

Therefore, the total uncertainty, $\sigma_{\rho}$, can be related to a given measurement duration. 
Table 4.2 Measurement durations in units of number of $100 \%$ relative efficiency HPGe detectors.time necessary to obtain stated statistical uncertainty in ${ }^{239} \mathrm{Pu}$ concnetrations in a single spent fuel pin.

\begin{tabular}{cccc}
\hline \hline $\begin{array}{c}I_{e} \\
(\mathrm{~mA})\end{array}$ & $\begin{array}{c}1 \% \\
(\text { det·yr })\end{array}$ & $\begin{array}{c}5 \% \\
(\text { det.day })\end{array}$ & FOM \\
\hline 0.01 & $4.5 \times 10^{8}$ & $6.5 \times 10^{9}$ & 0.03 \\
0.10 & $4.5 \times 10^{6}$ & $6.6 \times 10^{7}$ & 0.11 \\
1.0 & $5.5 \times 10^{3}$ & $8.0 \times 10^{4}$ & 0.31 \\
10 & 120 & $1.8 \times 10^{3}$ & 0.65 \\
45 & 15.4 & 225 & 0.86 \\
100 & 6.0 & 88 & 0.93 \\
\hline
\end{tabular}

\section{Prospects for Using HPGe Detectors}

Measurement durations necessary to obtain $1 \%$ and $5 \%$ uncertainties, in units of the product of number of detectors and time are tabulated in Table 4.2 for several beam intensities. The fourth column indicates the relative efficiency for a measurement with the stated beam current. The stronger the current, the less significant is the radioactive background. However, in the assumed configuration, currents above $45 \mathrm{~mA}$ will begin to induce problematic count rates in an HPGe detector.

The stated measurement times are for a single fuel pin. The measurement times for an exterior row fuel pin of an assembly would be quite similar if a detector were collimated to only view the irradiated portion of the pin. Fuel pins located in deeper rows in an assembly accrue statistics at slower rates due to self shielding by other pins. We therefore conclude that determination of ${ }^{239} \mathrm{Pu}$ concentration in spent fuel via measurement of backscattered bremsstrahlung-induced NRF $\gamma$ rays is too impractical to pursue.

\subsubsection{Alternative Backscatter Schemes}

The signal-to-noise ratio, $\xi$, in the backscatter measurements are well into the small $\xi$ regime, wherein statistical uncertainty in backscatter NRF-measured concentrations scale as $\sigma \propto 1 / \sqrt{N \xi}$. Given that the figure of merit in Table 4.2 does not decrease significantly below unity (i.e., $I_{e} \gtrsim 10$ $\mathrm{mA}$ ), the regime implies the following:

- Detectors that operate at higher (lower) count rates may proportionally increase (decrease) $N$ for a given measurement duration and therefore decrease (increase) $\sigma$ by $1 / \sqrt{N}$;

- Detectors with better (worse) resolution proportionally increase (decrease) $\xi$ for a given measurement and therefore decrease (increase) $\sigma$ for a given measurement duration by $1 / \sqrt{\xi}$;

- Measurement of a material with larger (smaller) NRF resonances produces proportionally larger (smaller) values of $N$ and $\xi$, thereby proportionally decreasing (increasing) $\sigma$ for a given measurement.

The last point indicates that ${ }^{240} \mathrm{Pu}$ concentrations will be easier to measure, assuming it has larger NRF resonances. However, unless ${ }^{240} \mathrm{Pu}$ has exceptionally strong resonances (which are not theoretically predicted), measurement times will not be reduced to practical durations. 


\subsection{Transmission NRF Assay}

The concept of performing a transmission measurement to determine $\mathrm{Pu}$ content in spent fuel was presented in Section 2.2.2. The method has the following advantages compared to a backscatter measurement:

- the positioning of radiation detectors makes it possible to shield them from radioactivity emitted from the spent fuel;

- measured NRF $\gamma$ rays have significantly improved signal strengths, relative to the nonresonant photon scatter background; and

- neglecting the small effects of notch refill, the measurement has uniform sensitivity throughout the volume of the irradiated portion of the fuel assembly.

Transmission methods also generally allow for stronger photon sources than those used in a backscatter measurement. Assuming intense CW electron sources, such as a Rhodotron, can be used to generate bremsstrahlung, the transmission measurement of entire fuel assemblies produces more effective measurements than single fuel pins.

This section uses the results of Sections 3.2.1, 3.2.2, and 3.2.5 to examine the effectiveness of a transmission measurement of $\mathrm{Pu}$ areal densities in spent fuel. We examine the precision at which the IOI content may be measured in spent fuel by way of a transmission NRF measurement using the range of $\mathrm{Pu}$ concentrations that are expected in spent fuel (see Section 2.2.4).

\subsubsection{Expected Precision for Measurement of Actinides in Spent Fuel using HPGe Detector Array}

In a transmission assay, the rate at which a particular resonance undergoes NRF in the TD is proportional to the product of the integrated cross section of that resonance, the expected photon intensity incident upon the TD at the resonance centroid energy without resonant attenuation, and the effective attenuation, $\mathbb{A}(N x)$. Using the $\mathrm{NRF}$ data for ${ }^{239} \mathrm{Pu}$ in Table 3.3 and assuming the incident bremsstrahlung photon spectrum after transport through spent fuel and incident on TD, given in Figure 3.2, we estimate relative count rates for each of the ${ }^{239} \mathrm{Pu}$ resonances, and use equation 3.29 to estimate the expected experimental uncertainty in a ${ }^{239} \mathrm{Pu}$ areal density measurement. Two examples are given in Table 4.3. The examples assume that four hundred $100 \%$ relative efficiency HPGe detectors are located behind lead filters of thickness $x_{f}$ and positioned $100 \mathrm{~cm}$ from a $4 \mathrm{~mm}$ thick TD. The uncertainties expected from a 24 hour measurement using $170 \mathrm{~mA}$ beam of $2.6 \mathrm{MeV}$ electrons are shown for $x_{f}=8 \mathrm{~cm}$ and for a $42 \mathrm{~mA}$ beam for $x_{f}=6$ $\mathrm{cm}$. Both cases result in the maximum total photon interaction rate in the HPGe detectors of $2 \times 10^{4} / \mathrm{s}$. In this geometry, $1 / 3$ of the total available backward solid angle is subtended by the detectors.

To estimate the total uncertainty of the areal density of ${ }^{239} \mathrm{Pu}$, we assume that the areal density value is determined from a weighted mean of the measured areal densities from each resonance. This estimate, using equations 4.2 - 4.5 is based solely on counting statistics and is given in the last row of Table 4.3 .

Other important $\mathrm{Pu}$ isotopes in $\mathrm{SNF}$ besides ${ }^{239} \mathrm{Pu}$ are ${ }^{240} \mathrm{Pu}$ and ${ }^{242} \mathrm{Pu}$. While the NRF responses of these isotopes have not been measured yet, nuclides with even numbers of neutrons 
Table 4.3 Expected effective attenuation values, $\mathbb{A}$, signal/background ratios of the NRF $\gamma$ rays, $\xi$, numbers of counted NRF $\gamma$ rays due to ${ }^{239} \mathrm{Pu}$ resonances, $C$, and statistical relative uncertainties, $\sigma_{\mathrm{Nx}}$, for two simulated assays of ${ }^{239} \mathrm{Pu}$ in spent fuel assemblies. The assay assumes a 24 hour measurement of a fuel assembly containing 0.25 $\mathrm{g} / \mathrm{cm}^{2}{ }^{239} \mathrm{Pu}$ and that the bremsstrahlung source induces $2 \times 10^{4}$ counts per second in each of 400 HPGe detectors. An * indicates that the corresponding NRF $\gamma$ ray is due to de-excitation of the NRF state to the first-excited state of ${ }^{239} \mathrm{Pu}$.

\begin{tabular}{cccclcl}
\hline \hline$E_{\text {level }}$ & \multirow{2}{*}{$\xi$} & \multicolumn{3}{c}{$x_{f}=6 \mathrm{~cm}$} & \multicolumn{2}{c}{$x_{f}=8 \mathrm{~cm}$} \\
$(\mathrm{MeV})$ & & & $C$ & $\sigma_{\mathrm{Nx}}(\%)$ & $C$ & $\sigma_{\mathrm{Nx}}(\%)$ \\
\hline 2.040 & 0.998 & 0.87 & $5.1 \times 10^{6}$ & 39.6 & $7.6 \times 10^{6}$ & 32.5 \\
$2.144^{*}$ & 0.996 & 0.59 & $2.3 \times 10^{6}$ & 33.8 & $3.4 \times 10^{6}$ & 27.7 \\
2.144 & 0.996 & 1.91 & $7.5 \times 10^{6}$ & 12.8 & $1.1 \times 10^{7}$ & 10.5 \\
2.289 & 0.998 & 1.91 & $3.7 \times 10^{6}$ & 41.4 & $5.5 \times 10^{6}$ & 34.0 \\
$2.432^{*}$ & 0.996 & 2.44 & $3.3 \times 10^{6}$ & 18.5 & $5.0 \times 10^{6}$ & 15.1 \\
2.432 & 0.996 & 2.72 & $3.0 \times 10^{6}$ & 19.0 & $4.5 \times 10^{6}$ & 15.6 \\
2.454 & 0.998 & 2.60 & $2.8 \times 10^{6}$ & 41.3 & $4.2 \times 10^{6}$ & 33.9 \\
2.464 & 0.998 & 2.34 & $2.4 \times 10^{6}$ & 51.6 & $3.6 \times 10^{6}$ & 42.3 \\
\hline total & & & $3.0 \times 10^{7}$ & 8.3 & $4.5 \times 10^{7}$ & 6.8 \\
\hline
\end{tabular}

and protons generally have stronger NRF resonances than those with at least one unpaired nucleon such as ${ }^{239} \mathrm{Pu}^{1}$. With this in mind, we estimate the accuracy to which a measurement of the ${ }^{240} \mathrm{Pu}$ content in a spent fuel assembly might be obtained. Assuming that the ${ }^{238} \mathrm{U}$ NRF state characteristics shown in Table 3.4 are representative of the ${ }^{240} \mathrm{Pu}$ resonances, we can calculate the total statistical uncertainty for the ${ }^{238} \mathrm{U}$ resonances assuming an areal density in spent fuel of 0.15 $\mathrm{g} / \mathrm{cm}^{2}$. The results from this calculation are shown in Table 4.4.

While measurement of ${ }^{239} \mathrm{Pu}$ areal densities in spent fuel to $1 \%$ precision appears to require $1.9 \times$ $10^{4}$ detector-days (or 3 times as long as in a backscatter measurement), a 1\% precision measurement of ${ }^{240} \mathrm{Pu}$ could take 260 detector.days, if ${ }^{240} \mathrm{Pu}$ has similar NRF intensities as those known for ${ }^{238} \mathrm{U}$. This demonstrates the importance of strong resonances for transmission measurements.

Thicker filters appear to enable faster accrual of counting statistics, however in the assumption that detection is rate-limited, increasing the filter thickness from 6 to $8 \mathrm{~cm}$ would imply that the electron beam intensity would increase from 42 to $170 \mathrm{~mA}$. Given the significant increase in heatload on the converter and power consumption, this may not be an attractive option.

\subsubsection{Measurement of $\mathrm{Pu}$ in Spent Fuel using Alternative Detector Types}

Whereas NRF $\gamma$ rays measured in backscatter measurements were determined to be significantly weaker than background photon intensities within any known detector's resolution, this is not the case for a transmission measurement. Because of this, worsening detector resolution does not reduce the precision of the measurement by $1 / \sqrt{\xi}$, but rather produces a more complicated and less severe effect. Conversely, detectors that can operate at higher count rates would allow significantly stronger beams and or thinner filters. We explicitly consider the use of $\mathrm{LaBr}_{3}$, which can easily tolerate $\sim 15$ times higher count rates than HPGe detectors, and evaluate two cases with

\footnotetext{
${ }^{1}$ See for example, references[34],[35], and[36]
} 
Table 4.4 Same as Table 4.3, except for $0.15 \mathrm{~g} / \mathrm{cm}^{2}{ }^{240} \mathrm{Pu}$ using the known ${ }^{238} \mathrm{U}$ resonances as example ${ }^{240} \mathrm{Pu}$ responses. The assay assumes a 24 hour measurement of a fuel assembly and that the bremsstrahlung source induces $2 \times 10^{4}$ counts per second in each of 400 HPGe detectors. An * indicates that the corresponding NRF $\gamma$ ray is due to de-excitation of the NRF state to the first-excited state of ${ }^{238} \mathrm{U}$.

\begin{tabular}{cccclcl}
\hline \hline$E_{\text {level }}$ & \multirow{A}{*}{$\xi$} & \multicolumn{3}{c}{$x_{f}=6 \mathrm{~cm}$} & \multicolumn{2}{c}{$x_{f}=8 \mathrm{~cm}$} \\
$(\mathrm{MeV})$ & & & $C$ & $\sigma_{\mathrm{Nx}}(\%)$ & $C$ & $\sigma_{\mathrm{Nx}}(\%)$ \\
\hline $2.176^{*}$ & 0.989 & 5.2 & $2.0 \times 10^{7}$ & 2.3 & $3.0 \times 10^{7}$ & 1.9 \\
2.176 & 0.989 & 8.1 & $3.1 \times 10^{7}$ & 1.8 & $4.7 \times 10^{7}$ & 1.5 \\
$2.209^{*}$ & 0.989 & 7.9 & $1.8 \times 10^{7}$ & 2.5 & $2.7 \times 10^{7}$ & 2.0 \\
2.209 & 0.989 & 8.0 & $3.0 \times 10^{7}$ & 1.9 & $4.4 \times 10^{7}$ & 1.6 \\
$2.245^{*}$ & 0.994 & 4.5 & $9.3 \times 10^{6}$ & 6.7 & $1.4 \times 10^{7}$ & 5.5 \\
2.245 & 0.994 & 7.3 & $1.5 \times 10^{7}$ & 5.0 & $2.2 \times 10^{7}$ & 4.1 \\
$2.295^{*}$ & 0.998 & 1.7 & $3.3 \times 10^{6}$ & 33 & $4.9 \times 10^{6}$ & 27 \\
2.295 & 0.998 & 2.9 & $5.5 \times 10^{7}$ & 23 & $8.2 \times 10^{6}$ & 19 \\
$2.410^{*}$ & 0.996 & 4.0 & $4.6 \times 10^{6}$ & 13 & $6.9 \times 10^{6}$ & 11 \\
2.410 & 0.996 & 6.5 & $7.4 \times 10^{7}$ & 9.9 & $1.1 \times 10^{7}$ & 8.1 \\
$2.468^{*}$ & 0.995 & 4.5 & $4.7 \times 10^{6}$ & 12 & $6.9 \times 10^{6}$ & 9.6 \\
2.468 & 0.995 & 7.4 & $7.7 \times 10^{7}$ & 8.6 & $1.1 \times 10^{7}$ & 7.1 \\
\hline total & & & $1.6 \times 10^{8}$ & 0.98 & $2.3 \times 10^{8}$ & 0.81 \\
\hline
\end{tabular}

$4 \mathrm{~cm}$ and $6 \mathrm{~cm}$ thick filters, respectively. The expected uncertainties for a 24-hour transmission measurement using $400 \mathrm{LaBr}_{3}$ detectors of similar size to $100 \%$ relative efficiency HPGe detectors were calculated. The beam intensities, $I_{e}$, corresponding to the given detector count rates and the resulting fractional areal density statistical uncertainties, $\sigma_{\mathrm{Nx}}$ for these geometries are presented in Table 4.5.

These results indicate that lower-resolution detectors capable of significantly higher count rates may be capable of providing 20\% - 40\% lower statistical error (higher precision) but for the price of a needed 10-fold increase in electron beam current. Better precision transmission measurements of $\mathrm{Pu}$ in spent fuel can be achieved, so long as bremsstrahlung beam intensity may be increased such that detectors are rate-limited.

\subsection{Pu Assay with Bremsstrahlung Sources Conclusions}

From the examples presented in this section, we draw the following conclusions:

- backscatter measurements of ${ }^{239} \mathrm{Pu}$ concentration in SNF are not practical, and it is unlikely that ${ }^{240} \mathrm{Pu}$ would have sufficiently strong NRF resonances to make backscatter measurements of ${ }^{240} \mathrm{Pu}$ practical;

- transmission measurements of ${ }^{239} \mathrm{Pu}$ concentration with bremsstrahlung would be even more time-consuming, requiring $\sim 1.9 \times 10^{4}$ detector.days to obtain $1 \%$ counting statistics;

- if ${ }^{240} \mathrm{Pu}$ resonances are as large as those in ${ }^{238} \mathrm{U}$, transmission measurements of ${ }^{240} \mathrm{Pu}$ con- 
Table 4.5 Summarizing the effects when using a 400 detector array for a 24-hour measurement on maximum beam current, $I_{e}$, to get the max count rate, $\dot{C}_{\max }$, and the resulting fractional areal density statistical uncertainties, $\sigma_{\mathrm{Nx}}$, when a $\mathrm{LaBr}_{3}$ detector array is used instead of $\mathrm{HPGe}$. The $*$ indicates that ${ }^{240} \mathrm{Pu}$ resonances are unknown at that known ${ }^{238} \mathrm{U}$ resonances were used for this calculation instead.

\begin{tabular}{|c|c|c|c|c|c|}
\hline & & \multicolumn{2}{|c|}{ HPGe } & \multicolumn{2}{|c|}{$\mathrm{LaBr}_{3}$} \\
\hline & $\sigma_{E}$ & \multicolumn{2}{|c|}{$1.3(\mathrm{keV})$} & \multicolumn{2}{|c|}{$23(\mathrm{keV})$} \\
\hline & $\dot{C}_{\max }$ & \multicolumn{2}{|c|}{$2 \times 10^{4} / \mathrm{s}$} & \multicolumn{2}{|c|}{$3 \times 10^{5} / \mathrm{s}$} \\
\hline & $x_{f}$ & $4 \mathrm{~cm}$ & $6 \mathrm{~cm}$ & $4 \mathrm{~cm}$ & $6 \mathrm{~cm}$ \\
\hline & $I_{e}(\mathrm{~mA})$ & 7.8 & 42 & 117 & 630 \\
\hline${ }^{239} \mathrm{Pu}$ & $\sigma_{\mathrm{Nx}}(\%)$ & 11.4 & 8.3 & 9.3 & 6.53 \\
\hline${ }^{240} \mathrm{Pu}^{*}$ & $\sigma_{\mathrm{Nx}}(\%)$ & 1.4 & 0.98 & 1.0 & 0.54 \\
\hline
\end{tabular}

centration to $1 \%$ uncertainty would still take $\sim 200$ detector days, which is possible even if not practical. Measurements with a statistical uncertainty of several percent would only take $\sim 1 / 10$ of the time and reduce measurement time and/or the size of the detector array accordingly;

- filter thicknesses are large for backscatter measurements due to SNF radioactivity, which remains sufficiently intense after $10 \mathrm{y}$;

- for transmission measurements, increasing filter thicknesses from 4 to $8 \mathrm{~cm}$ can slightly improve counting times with significant increase in necessary beam intensity; and

- detectors that can operate at significantly higher count rates at the small expense of resolution may yield shorter counting times for transmission measurements. 


\section{Determining $\mathrm{Pu}$ in Spent Fuel Using Quasi-monoenergetic Photon Sources}

The ideal photon source for an NRF measurement would be a monoenergetic beam that excites a particular resonance of interest and generates a minimal scattered photon background. In contrast, bremsstrahlung sources produce a continuous energy spectrum whose intensity falls off exponentially up to the energy of the electron beam. Quasi-monoenergetic (QM) photon sources produce significantly narrower source photon energy ranges. Sources based on the Compton upscattering of laser light are under development to produce intense photon beams with $1-10 \mathrm{keV}$ energy width. This source type is briefly described in Section 2.3.1.

In our analysis of NRF systems using QM sources it is mostly assumed that the source delivers a continuous beam. However, laser Compton sources that have been produced are pulsed. If the pulse rate of a hypothetical source is sufficiently high, it may be considered approximately continuous. Lower frequency sources require more detailed consideration and would best be combined with threshold (Cherenkov) detectors as discussed at the end of this section.

\subsection{Backscatter Measurement Using Quasi-monoenergetic Photons}

Analogous to Section 4.1, we consider here the possibility of directly measuring the rate at which $\mathrm{Pu}$ in spent fuel undergoes NRF when irradiated with a QM photon source. The reduction in photon intensity and energy at large scatter angles again motivates measurement at primarily backwards angles, i.e., $\theta>90^{\circ}$. To study this measurement scenario, we divide the problem into four parts:

- Backscatter of resonant-energy photons;

- Backscatter of non-resonant photons;

- Radioactive background due to spent fuel activity; and

- Detector response.

\subsubsection{Backscattered Photon Spectra}

We assume a QM beam that is uniform in intensity over an $8 \mathrm{eV}$ range and sufficiently narrow to be considered incident upon only a single row of fuel pins in an assembly. The mean energy of the beam was taken to be the centroid energy of the $2431 \mathrm{keV}{ }^{239} \mathrm{Pu}$ resonance. Likewise, we assume HPGe detectors are collimated and positioned to view only a single fuel pin within the irradiated row of fuel pins. To simulate this, the probability of photons interacting within fuel pins that are outside of the collimator viewing angle were explicitly excluded from directly contributing to the calculated photon flux. A schematic of the geometry simulated in MCNPX is shown in Figure 5.1. The portions of the fuel lattice that are differently colored indicate regions within which photons interacting are considered to directly contribute to the calculated flux. 


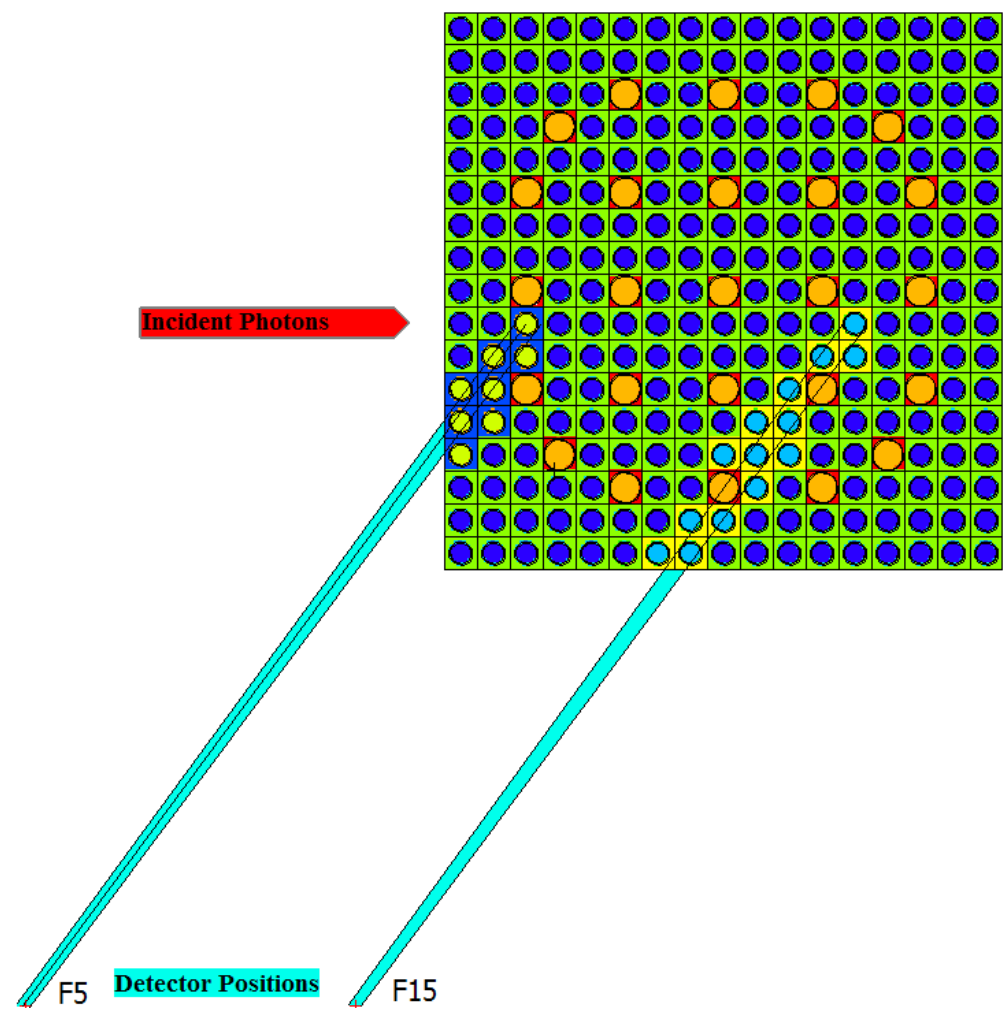

Figure 5.1 Geometry simulated in MCNPX to determine energy-dependent flux of backscattered QM photons incident upon a spent fuel assembly.

The resonantly and non-resonantly backscattered photon fluences were calculated with identical MCNPX runs, except NRF datafiles were excluded from the non-resonant photon scatter calculations. The photon source is assumed to be non-diverging, normally incident upon the fuel assembly, centered at the row of pins adjacent to the center row (which has several void locations), and uniformly distributed throughout a $6.3 \mathrm{~mm}$ radius circle.

Two tallies were performed, each giving the expected photon fluence at a hypothetical detector location. Both locations were $100 \mathrm{~cm}$ from the row of fuel pins that were irradiated, at an angle of $125^{\circ}$ from the initial beam trajectory. One tally simulated a detector collimated to view the 3rd fuel pin in the row of irradiated fuel, and the second simulated a detector collimated to view the 13th fuel pin. The spectra calculated at each detector position are shown in Figures 5.2 and 5.3, where the blue lines indicate the spectrum resulting when NRF physics is included, and the red when NRF is excluded. The reason that a full energy peak remains when NRF is disabled is due to the fact that non-resonant elastic scatter (NRES) is still possible. The cross section for NRES is significantly smaller than that for NRF, however all isotopes in the spent fuel are capable of undergoing NRES, whereas presumably, only the ${ }^{239} \mathrm{Pu}$ at 0.4 wt.\% undergoes NRF at this energy. At full energy, the calculated intensities of NRF $\gamma$ rays divided by photon intensities calculated without NRF is 7.2 for both pins. This value is roughly the maximum possible signalto-background ratio, $\xi_{\text {max }}$, that a $\mathrm{QM}$ backscatter measurement of the $2431 \mathrm{keV}{ }^{239} \mathrm{Pu}$ resonance could attain. However, most QM photon sources are at least 100 times broader in energy, resulting in signficantly worse $\xi$. For $\sigma_{S}>\Delta E_{\text {det }}$, realistic signal-to-background ratios, $\xi$, using QM photons 


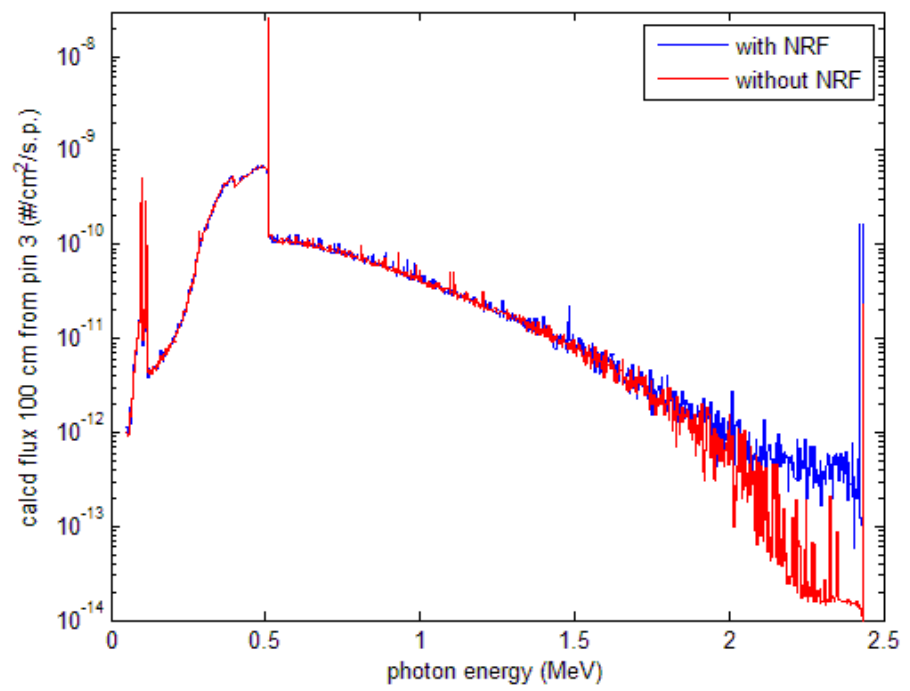

Figure 5.2 MCNPX-computed spectrum due to backscatter of monoenergetic photons at resonant (blue) and a nearby non-resonant (red) energies incident upon a single row of a spent fuel assembly. These spectra correspond to the labeling 'F5' in Figure 5.1.( $3^{\text {rd }}$ pin)

Table 5.1 Parameters describing average spectra due to a single monoenergetic photon incident upon a spent fuel assembly with resonant energy that are backscattered toward detectors that are collimated to view the 3rd and 13th row of pins, respectively. $\frac{\Phi(E<511)}{\Phi_{T}}$ and $\frac{\Phi(E=511)}{\Phi_{T}}$ indicate the fraction of the total calculated flux that are due to photons with energies less than, and equal to $511 \mathrm{keV}$, respectively. $\Phi_{\mathrm{NRF}}$ is the expected flux of NRF $\gamma$ rays per source photon, $\Phi_{\mathrm{ES}}$ is the expected flux due to non-resonant elastic scatter of source-energy photons, and $\Phi_{T}$ is the expected total flux of photons due to monoenergetic, monodirectional photons normally incident upon a 1 $\mathrm{cm}$ diameter portion of a fuel assembly.

\begin{tabular}{cccccc}
\hline \hline $\begin{array}{c}\text { tally } \\
\text { pin }\end{array}$ & $\frac{\Phi(E<511)}{\Phi_{T}}$ & $\frac{\Phi(E=511)}{\Phi_{T}}$ & $\begin{array}{c}\Phi_{\mathrm{NRF}} \\
\left(1 / \mathrm{cm}^{2} / \mathrm{ph}\right)\end{array}$ & $\begin{array}{c}\Phi_{\mathrm{ES}} \\
\left(1 / \mathrm{cm}^{2} / \mathrm{ph}\right)\end{array}$ & $\begin{array}{c}\Phi_{T} \\
\left(1 / \mathrm{cm}^{2} / \mathrm{ph}\right)\end{array}$ \\
\hline 3 & 0.44 & 0.33 & $3.1 \times 10^{-10}$ & $2.4 \times 10^{-11}$ & $7.83 \times 10^{-8}$ \\
13 & 0.80 & 0.05 & $2.7 \times 10^{-12}$ & $2.0 \times 10^{-13}$ & $7.96 \times 10^{-9}$ \\
\hline
\end{tabular}

are determined by the detector resolution. The ratio can be estimated as

$$
\xi_{\text {real }} \approx \frac{\xi_{\text {MonoeE }} \Delta E_{\text {src }}}{\Delta E_{\mathrm{det}}} \approx \frac{1}{50}
$$

for these simulations, in which $\Delta E_{s r c}=8 \mathrm{eV}$ and $\Delta E_{\operatorname{det}}=3 \mathrm{keV}$ HPGe detector. The ramifications of poor signal-to-background will be discussed below.

Values describing the total calculated spectra for these runs are given in Table 5.1. The column labeled $\Phi_{\mathrm{ES}}$ is the intensity of the full-energy peak in the simulation that excluded NRF physics, $\Phi_{\mathrm{NRF}}$ is the sum of photons that are expected to be due to NRF, $\Phi_{\mathrm{NRF}}=\Phi(E=2431)+\Phi(E=$ $2423)-\Phi_{E S}$. The fraction of the total flux that is due to photons of energy $511 \mathrm{keV}$ or less is quite large. This implies that if necessary, absorbers could be placed between the fuel assembly and photon detectors to reduce the low-energy photon count rate and therefore the total count rate of a detector. 


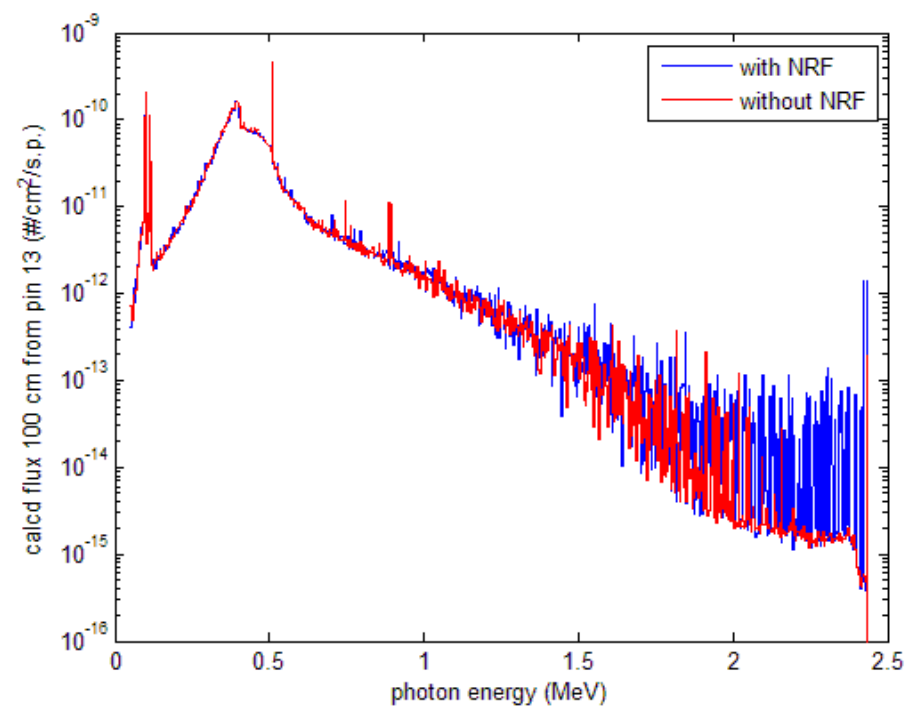

Figure 5.3 MCNPX-computed spectrum due to backscatter of monoenergetic photons at resonant (blue) and a nearby non-resonant (red) energies incident upon a single row of a spent fuel assembly. These spectra correspond to the labeling 'F15' in Figure 5.1. ( $13^{\text {th }}$ pin)

Neglecting the contribution to the background count rate due to radioactive decay of spent fuel, we may use the values in Table 5.1 to determine the best-case counting parameters necessary to make a measurement of ${ }^{239} \mathrm{Pu}$ content in spent fuel using backscattered $\mathrm{NRF} \gamma$ rays. For simplicity, we assume that photons will be detected with a $100 \%$ relative efficiency HPGe detector of surface area, $A_{d e t}=50 \mathrm{~cm}^{2}$. To make a $1 \%$ measurement, we would normally require $10^{4} \mathrm{NRF}$ counts. However, due to the very poor signal intensity, relative to background $(\xi=1 / 50)$, we estimate using equation 2.23 that $C_{\mathrm{NRF}}=10^{5} \mathrm{NRF}$ counts are necessary. Given that NRF $\gamma$ rays have a full-energy deposition probability for the detector, $\epsilon_{\mathrm{NRF}}=0.2$, we would need

$$
N_{\text {res }}=\frac{C_{\mathrm{NRF}}}{\epsilon_{\mathrm{NRF}} A_{\text {det }} \Phi_{\mathrm{NRF}}}=3.22 \times 10^{13}
$$

photons within $\pm 4 \mathrm{eV}$ of the resonance centroid energy to make this measurement on a fuel pin in the third layer with a single HPGe detector. Likewise, a 1\% measurement of a pin in the 13th row of fuel would require $3.70 \times 10^{15}$ photons within $\pm 4 \mathrm{eV}$ of the resonance centroid energy. For example a proposed (LLNL[16]) QM photon source is to generate $10^{6}$ photons $/ \mathrm{eV} / \mathrm{s}$. With this source, a $1 \%$ measurement of the $3 \mathrm{rd}$ row of fuel pins would require $4 \times 10^{6} \mathrm{~s}$ of active detectors, or approximately 1200 detector·hours.

To this point, detector count rate limitations have been neglected. We assume that the photon source energy distribution can be described by a Gaussian distribution with width $\sigma_{S}$ :

$$
P(E)=\frac{1}{\sqrt{2 \pi \sigma_{S}^{2}}} \exp \left[\frac{-\left(E-E_{C}\right)^{2}}{2 \sigma_{S}^{2}}\right]
$$

and that the source is well-tuned, i.e., the centroid energy of the source is equal to the centroid energy of the resonance, $E_{C}=E_{\text {res }}$. With these assumptions, the total number of photons emitted 
from a Gaussian-distributed source is given by:

$$
N_{T}=\frac{N_{\text {res }}}{\int_{E_{C}-4 e V}^{E_{C}+4 e V} P(E) d E} \approx \frac{\sqrt{2 \pi} N_{\text {res }} \sigma_{S}[\mathrm{eV}]}{8 \mathrm{eV}}
$$

when $\sigma_{S} \gg 8 \mathrm{eV}$. Likewise, we assume that the probability for detection of a photon that is directed toward the detector is unity (although the photon may not deposit its full energy). We can then determine the total number of counts a detector will measure, for a given number of NRF counts.

$$
C_{T}=N_{T} A_{d e t} \Phi_{T}
$$

Substituting Equation 5.4 into 5.5, we have the total number of counts expected in a detector for a photon source energy width and a requisite number of resonant-energy photons.

$$
C_{T}=\frac{C_{\mathrm{NRF}} \Phi_{T}}{\epsilon_{\mathrm{NRF}} \Phi_{\mathrm{NRF}}} \frac{\sqrt{2 \pi} \sigma_{S}}{8[\mathrm{eV}]}
$$

This can be related to the minimum time, $t$, a measurement would require, assuming a system of $n_{\text {det }}$ detectors, each operating at the maximum tolerable detection rate, $\dot{C}_{\max }$.

$$
t=\frac{C_{T}}{\dot{C}_{\max } n_{\text {det }}}
$$

Likewise, we can determine the intensity, $\dot{S}$, of the QM photon beam necessary to induce NRF at the maximum rate, thereby minimizing the required measurement time for a given measurement geometry and precision,

$$
\dot{S}=\frac{\dot{C}_{\max }}{\Phi_{T} A_{d e t}}
$$

Equations 2.23, and 5.6 - 5.8 essentially constrain the parameters of a non-destructive determination of isotopic compositions using measured backscattered NRF. For all known minor actinide resonances in spent fuel, the signal will be significantly weaker than the background, therefore the number of NRF counts necessary to make a measurement of a given precision scales as $1 / \sqrt{\xi}$. Likewise, the number of non-resonant counts will scale with the beam width. For beams with realistic energy bandwidth, this begins to constrain the total count rate at which detectors may operate. A summary of the constraining parameters of such a system is shown in Table 5.2 for various source widths and HPGe detectors (assuming $3 \mathrm{keV}$ resolution).

Revisiting the possible use of the proposed (LLNL) quasi-monoenergetic photon source, and assuming $\sigma_{S} \approx 2500 \mathrm{eV}$, we observe that approximately 63 detector.days would be necessary to perform the $1 \%$ meaurement, indicating this measurement would be possible to conduct in 1 day using a 63 HPGe detector array. Likewise, more intense photon sources (in terms of total number of photons on target per second) would generally saturate unshielded HPGe detectors, which would necessitate the use of a filter, which is discussed below in the context of filtering spent fuel radioactivity.

\subsubsection{Influence of Spent Fuel Radioactivity on Backscatter Measurement}

The probable photon intensity to which radiation detectors would be subjected due to radioactive decay of used fuel when making a direct measurement of backscattered NRF radiation is estimated 
Table 5.2 Backscatter measurement parameters for $1 \%$ measurment of ${ }^{239} \mathrm{Pu}$ in spent fuel assembly for third row of pins, neglecting fuel radioactivity. HPGe detectors have been assumed to have $3 \mathrm{keV}$ resolution and to operate at $2 \times 10^{4} \mathrm{~Hz}$.

\begin{tabular}{ccccccc}
\hline \hline $\begin{array}{c}\sigma_{S} \\
(\mathrm{keV})\end{array}$ & $\begin{array}{c}\Phi_{\mathrm{NRF}} \frac{N_{T}}{N_{\mathrm{NRF}}} \\
\left(1 / \mathrm{cm}^{2} / \mathrm{ph}\right)\end{array}$ & $\xi$ & $\begin{array}{c}C_{\mathrm{NRF}} \\
\text { counts }\end{array}$ & $\begin{array}{c}C_{T} \\
\text { counts }\end{array}$ & $\begin{array}{c}t_{\text {meas }} \\
(\text { det.day })\end{array}$ & $\begin{array}{c}\text { intensity } \\
(1 / \mathrm{eV} / \mathrm{s})\end{array}$ \\
\hline 0.1 & $1.0 \times 10^{-11}$ & 0.42 & $2.4 \times 10^{4}$ & $9.5 \times 10^{8}$ & 0.55 & $2.0 \times 10^{7}$ \\
1 & $1.0 \times 10^{-12}$ & 0.042 & $7.0 \times 10^{4}$ & $2.8 \times 10^{10}$ & 15.6 & $2.0 \times 10^{6}$ \\
2.5 & $4.0 \times 10^{-13}$ & 0.017 & $1.1 \times 10^{5}$ & $1.1 \times 10^{11}$ & 63 & $8.2 \times 10^{5}$ \\
3 & $3.3 \times 10^{-13}$ & 0.014 & $1.2 \times 10^{5}$ & $1.4 \times 10^{11}$ & 82 & $6.8 \times 10^{5}$ \\
10 & $1.0 \times 10^{-13}$ & 0.014 & $1.2 \times 10^{5}$ & $4.7 \times 10^{11}$ & 274 & $2.0 \times 10^{5}$ \\
100 & $1.0 \times 10^{-14}$ & 0.014 & $1.2 \times 10^{5}$ & $4.7 \times 10^{12}$ & 2740 & $2.0 \times 10^{4}$ \\
\hline
\end{tabular}

Table 5.3 Backscatter measurement parameters for $1 \%$ measurment of ${ }^{239} \mathrm{Pu}$ in spent fuel assembly for third row of pins, when 3 inch $\mathrm{Pb}$ filter is present. HPGe detectors have been assumed to have $3 \mathrm{keV}$ resolution and to operate at $10^{4} \mathrm{~Hz}$ due to beam photons and $10^{4} \mathrm{~Hz}$ due to radioactive decay in spent fuel.

\begin{tabular}{ccccccc}
\hline \hline $\begin{array}{c}\sigma_{S} \\
(\mathrm{keV})\end{array}$ & $\begin{array}{c}\Phi_{\mathrm{NRF}} \frac{N_{T}}{N_{\mathrm{NRF}}} \\
\left(1 / \mathrm{cm}^{2} / \mathrm{ph}\right)\end{array}$ & $\xi$ & $\begin{array}{c}C_{\mathrm{NRF}} \\
\text { counts }\end{array}$ & $\begin{array}{c}C_{T} \\
\text { counts }\end{array}$ & $\begin{array}{c}t_{\text {meas }} \\
(\text { det.day })\end{array}$ & $\begin{array}{c}\text { intensity } \\
(1 / \mathrm{eV} / \mathrm{s})\end{array}$ \\
\hline 0.1 & $1.0 \times 10^{-11}$ & 0.42 & $2.4 \times 10^{4}$ & $9.5 \times 10^{8}$ & 0.07 & $6.1 \times 10^{9}$ \\
1 & $1.0 \times 10^{-12}$ & 0.042 & $7.0 \times 10^{4}$ & $2.8 \times 10^{10}$ & 2.1 & $2.0 \times 10^{8}$ \\
2.5 & $4.0 \times 10^{-13}$ & 0.017 & $1.1 \times 10^{5}$ & $1.1 \times 10^{11}$ & 8.4 & $2.4 \times 10^{8}$ \\
3 & $3.3 \times 10^{-13}$ & 0.014 & $1.2 \times 10^{5}$ & $1.4 \times 10^{11}$ & 11 & $2.0 \times 10^{8}$ \\
10 & $1.0 \times 10^{-13}$ & 0.014 & $1.2 \times 10^{5}$ & $4.7 \times 10^{11}$ & 37 & $6.1 \times 10^{7}$ \\
100 & $1.0 \times 10^{-14}$ & 0.014 & $1.2 \times 10^{5}$ & $4.7 \times 10^{12}$ & 366 & $6.1 \times 10^{6}$ \\
\hline
\end{tabular}

in Appendix B. The assay geometry depicted in Figure 5.1 is quite similar to that considered in Section B.1.1. In this section, it was determined that approximately $7.5-10 \mathrm{~cm}$ of $\mathrm{Pb}$ would need to be placed in the collimator opening between an HPGe detector and the portion of the interrogated fuel assembly that is within the line of sight. $7.5 \mathrm{~cm}$ of $\mathrm{Pb}$ will attenuate $2431 \mathrm{keV}$ photons by a factor of 40, and the total count rate due to scattered interrogating photons by a factor of 600. With this filter, we re-present the measurement parameters in Table 5.3. For sources with a broader energy width, the signal-to-background ratios are not significantly changed and measurement times actually decrease due to filtering of the low-energy portion of the backscattered spectrum. However, the necessary source intensity to achieve the tabulated rates are increased by a factor of approximately 40 .

The expected count rate of background photons that a $100 \%$ relative efficiency detector in this geometry, located behind $7.5 \mathrm{~cm}$ of $\mathrm{Pb}$, measures is approximately $10^{4} / \mathrm{s}$. The previously referred to QM photon source, producing $10^{6}$ photons $/ \mathrm{eV} / \mathrm{s}$ on target, would now induce $\sim 30$ counts $/ \mathrm{s}$ in a reference detector and $6 \times 10^{-4} \mathrm{NRF}$ counts/s. Therefore the assumption in the preceeding section that detector count rates are satuarated due to counting of interrogating source photons would require a significantly more intense photon beam, as indicated in Table 5.3. The expected count rate of radioactive decay photons contributing background at $2431 \mathrm{keV}$ would contribute a count rate of $3 \times 10^{-3}$ counts $/ 3 \mathrm{keV} / \mathrm{s}$. This would further decrease the signal-to-background 


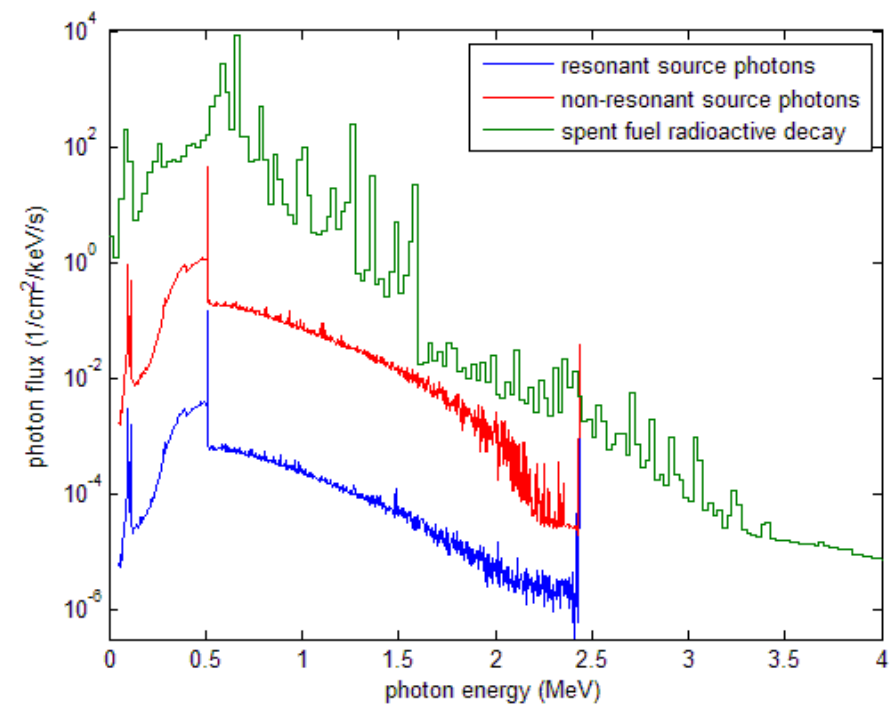

Figure 5.4 Relative intensities of photon fluxes due to scattering of resonant (blue) and non-resonant (red) interrogating source photons compared with the intensity of background photons (green).

ratio $\xi$ for the NRF signal (from 0.017 to 0.015 ) for proposed sources, but would have very little effect on $\xi$ for very intense sources. The time that the $1 \%$ measurement would take using the LLNL-proposed source would then increase by a factor of 43 from 50 detector.days to $2.2 \times 10^{3}$ detector·days.

\subsubsection{Measurement of Other Rows of Pins}

The preceding discussion has assumed a measurement of the 3rd row of fuel pins in an assembly. Flux calculations were also performed for the 1st, 7 th and 13 th rows of pins. The relative rates at which NRF $\gamma$ rays would be measured for an interrogating beam of the same intensity for these three rows are $4.5,1 / 5.5$, and $1 / 116$, respectively. These values indicate that a measurement of the first row of pins would require less time than indicated here, and that measurement of the innermost pins would require approximately 6 times longer measurements than indicated. The 13th row of pins (and presumably deeper rows) would be more efficiently measured by rotating the assembly and irradiating its back side.

\subsubsection{Use of Threshold Detectors}

Radiation detectors that are only sensitive to photons above a threshold energy do not necessarily significantly improve the prospect of making a measurement of ${ }^{239} \mathrm{Pu}$ content in spent fuel via a backscatter measurement unless the detector can also resolve the energy of detected photons, or can be used with a pulsed QM photon source to reduce the background rates through time-gating.

Without time gating, the issue of background can be illustrated by comparing the photon spectra shown in Figure 5.2 with a simulated spectrum of the emitted $\gamma$-ray spectrum from the radioactivity in a SNF assembly as is shown in Figure 5.4.

Threshold detectors effectively integrate the photon flux above some minimum energy $E_{\min }$, 
possibly with a response function, which to first order can be considered constant.

$$
C_{\mathrm{Th}} \approx \int_{E_{\min }}^{\infty} R(E) \Phi(E) d E
$$

The most optimistic threshold detector response model would be that of a step function and $E_{\text {min }}=2.4 \mathrm{MeV}$. In this case, the NRF signal would represent just $1.8 \times 10^{-4}$ of the measured signal, implying that statistics would accrue about 100 times slower than if the threshold detector primarily measured NRF signals, with most of the signal coming from the radioactive background and the non-resonant source photons.

However, if the QM photon source is pulsed, like the QM sources described in Section 2.3.1 with 10 ps bunches at $f=120 \mathrm{~Hz}$, detectors that measure single photons will be limited to very low acquisition rates. Conversely, integrating threshold-type detectors could be configured to measure an integrated signal only during the beam pulse. Assuming a time resolution $\sigma_{t}$ of $1 \mathrm{~ns}$, the measured intensity of radioactive background photons would be reduced by a factor of $\sigma_{t} \times f=1.2 \times 10^{-7}$. In this case, systems with threshold detectors and pulsed QM photon sources would measure a negligible radioactive background and would thus be able to measure spent fuel. Depending on the sharpness of the threshold that could be implemented, the signal-to-background ratios could reach those indicated for $\sigma_{S} \leq 3 \mathrm{keV}$ in Table 5.2. QM sources with a wider energy spread would result in proportionally smaller values of $\xi$.

\subsubsection{Conclusions for Backscatter Measurements Using Quasi-monoenergetic Sources}

The best-case scenario for using NRF to measure $\mathrm{Pu}$ content in spent fuel is to use a truly monoenergetic photon source of arbitrary intensity and a detector with good energy resolution that is not count rate-limited. In this case, the NRF signal due to the strongest ${ }^{239} \mathrm{Pu}$ resonances is approximately 10 times background (for $3 \mathrm{keV}$ energy resolution detectors), and measurement of $10^{4} \mathrm{NRF}$ counts would provide $\sim 1 \%$ counting statistics. In the geometry in which the detector-TD distance was $100 \mathrm{~cm}$, as described in Section 5.1, this would require $8 \times 10^{11}$ source photons for a single $100 \%$ relative efficiency detector to measure the third row of pins. More detectors would proportionally reduce the necessary number of source photons, i.e., an array of 100 such detectors would require $8 \times 10^{9}$ source photons.

This ideal scenario is unrealistic in three ways: energy-resolving detectors are incapable of measuring arbitrary count rates and photon sources can neither be arbitrarily intense, nor truly monoenergetic. The problem of detector rate limitations becomes foremost because it limits source beam intensities and necessitates shielding from the spent fuel radioactivity, which causes more source photons to be required to obtain the same number of NRF counts. Photon sources with broader energy distributions reduce the signal-to-background ratio, requiring longer counting times to obtain the same statistics.

If the QM source is pulsed, energy-resolving detectors may operate at average rates that are significantly lower than the nominal rate limit, resulting in increased counting times. Threshold detectors are not rate limited, and could be gated to reject radioactive background photons between photon source pulses. This could result in significant improvements for backscatter measurements using very intense QM sources. The best-case scenario would be the use of a $\sim 10^{8}$ photons $/ \mathrm{eV} / \mathrm{s}$, $\sigma_{S}=2.5 \mathrm{keV}$ source inducing $\mathrm{NRF}$ that is measured by an ideal Cherenkov detector array. With $\xi=0.017$, this measurement would take 12 hours using a single detector with a $50 \mathrm{~cm}^{2}$ 
area and an efficiency comparable to the full-energy efficiency of HPGe $(\sim 20 \%)$. An array of 80 such detectors could then accomplish a measurement with $1 \%$ statistical uncertainty in 10 minutes. While this indicates the promise of NRF, neither the detectors nor the photon source presently exist. Construction of demonstration QM sources of $10^{6}$ photons $/ \mathrm{eV} / \mathrm{s}$ intensity have been proposed, and aerogel Cherenkov materials could potentially produce acceptible threshold characteristics[37, 38].

In summary, we observe the following for backscatter measurements:

- Increasing the QM source intensity from the proposed LLNL design to $2 \times 10^{8} \mathrm{ph} / \mathrm{eV} / \mathrm{s}$ would proportionally reduce the necessary count time. Above this intensity, detector count rates would become the limiting factor and increased source intensities would require additional filter thicknesses to reduce count rates;

- Narrower than $2.5 \mathrm{keV}$ bandwidth beams would represent an improvement in $\xi$ and could potentially produce up to a factor of 8 decrease in measurement time;

- Broader bandwidth beams would not necessarily worsen the system performance, but would reduce the allowable increase in beam intensity before which additional filter thickness would be necessary, thus leading to longer measurement times;

- Improved detector energy resolution (over the $3 \mathrm{keV} \mathrm{FWHM} \mathrm{resolution} \mathrm{assumed} \mathrm{here)} \mathrm{would}$ result in proportionally better values of $\xi$, and proportionally shorter measurement times (until $\sim 5 \mathrm{eV}$ resolution is achieved);

- Worsened detector energy resolution results in proportionally longer measurement times until separate peaks cannot be resolved, (Table B.2 provides known background peaks and Table 3.3 provides known Pu NRF lines);

- Detectors capable of higher measurement rates proportionally decrease counting times and reduces necessary filter thickness, which further reduces counting times but also reduces the allowable increase in beam intensity or width;

- Measurements with gated threshold detectors and a pulsed photon source would be insensitive to the radioactive background and would thus be suitable for backscatter assay.

\subsection{Transmission Measurements Using Quasi-monoenergetic Sources}

Because the transmission measurements rely on a TD, the radiation detectors are not directly exposed to the large photon intensity due to radioactive decay in the used fuel assembly. Therefore we are able to consider the transmission measurement of a fuel assembly without directly considering the influence of the radioactive background. Analogously to Section 5.1, we divide the problem of measuring used fuel into several independent calculations:

1. Transport of resonant-energy photons;

2. Transport of non-resonant photons;

3. Detector response. 


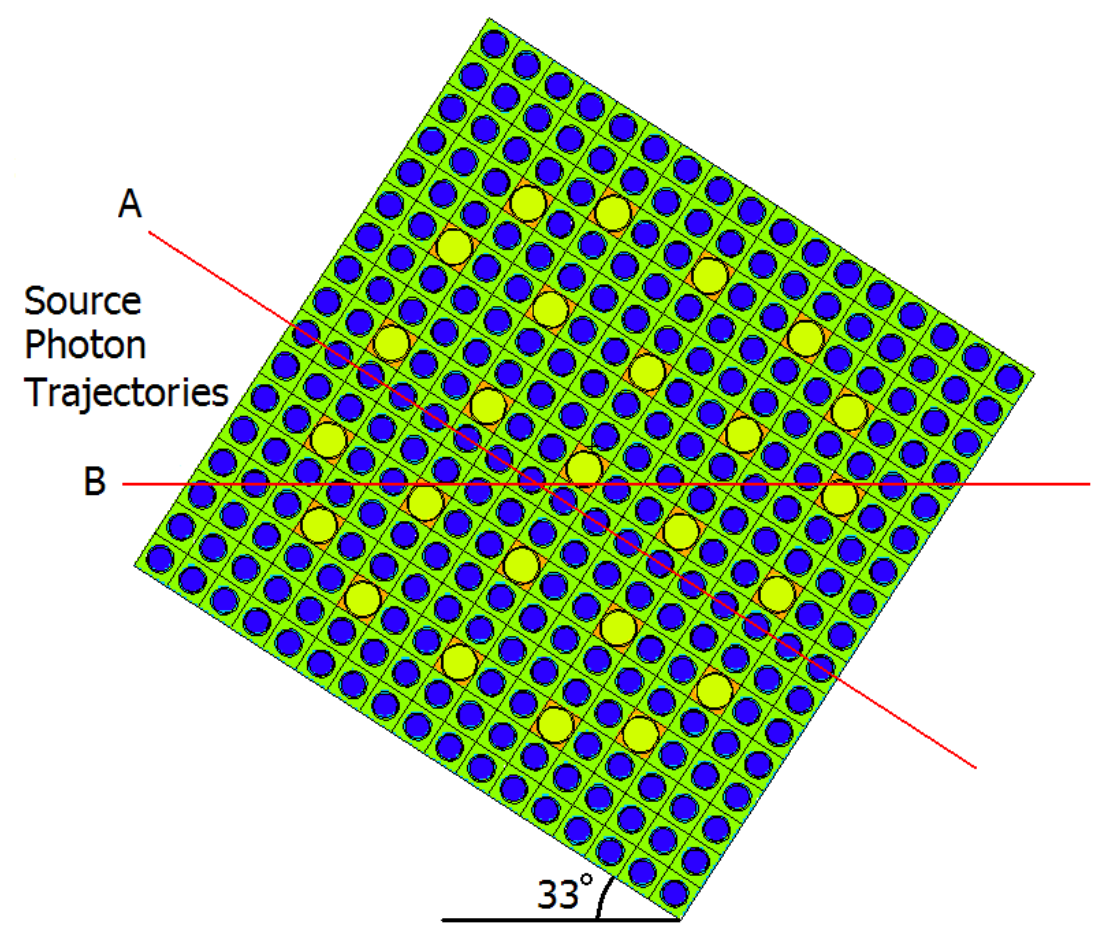

Figure 5.5 Schematic describing the source photon trajectories for the two different geometries of monoenergetic transmission measurements considered. The red lines indicate rays describing the trajectory of the source photons for the two geometries, labeled "A" and "B".

Table 5.4 Thicknesses of materials traversed by rays depicting monodirectional source trajectories in two source geometries.

\begin{tabular}{rcc}
\hline \hline $\begin{array}{r}\text { Source } \\
\text { Geometry }\end{array}$ & $\begin{array}{c}\mathrm{UO}_{2} \\
(\mathrm{~cm})\end{array}$ & $\begin{array}{c}\text { Zr Clad } \\
(\mathrm{cm})\end{array}$ \\
\hline $\mathrm{A}$ & 13.940 & 2.210 \\
$\mathrm{~B}$ & 7.654 & 2.700 \\
\hline
\end{tabular}

The transport of resonant and non-resonant photons were simulated in MCNPX in two assay geometries, both of which use monodirectional, monoenergetic photons to irradiate fuel in a $17 \times 17$ fuel assembly. In one geometry, the photon source is normally incident upon a full row (17 pins) adjacent to the center of the fuel assembly (labeled "A" in Figure 5.5). In the other geometry, the photon beam is assumed incident upon the assembly at an angle, $33^{\circ}$ from normal (labeled "B" in Figure 5.5). The red lines in Figure 5.5 represent the trajectories of the source photons through a fuel assembly for these two source geometries. The thicknesses of material through which the simulated photons penetrate as they traverse the fuel (assuming no interaction) are given in Table 5.4 .

Beyond the fuel assembly, a TD is placed in the trajectory of the interrogating photon beam. For these simulations, the TD was assumed to be a $1 \mathrm{~cm}$ thick plate of pure ${ }^{239} \mathrm{Pu}$ with a $10 \mathrm{~cm}$ 


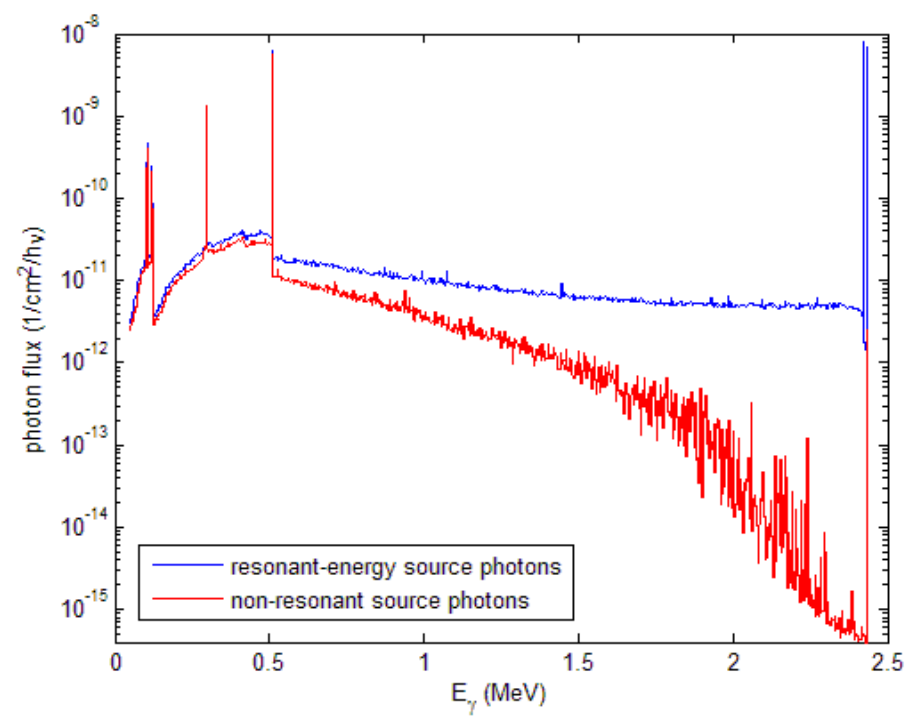

Figure 5.6 MCNPX-computed spectra due to a transmission measurement of a fuel assembly containing no ${ }^{239} \mathrm{Pu}$ using monoenergetic photons at resonant (blue) and a nearby non-resonant (red) energies incident upon a spent fuel assembly as indicated by Geometry "B" in Figure 5.5.

diameter ${ }^{1}$. In a real system the diameter of the TD would be as small as the transmitted beam. Finally, the photon flux, resulting from backscattered photons emitted from the TD is determined at a position $100 \mathrm{~cm}$ along a ray leaving the center of the $\mathrm{TD}$ at a $120^{\circ}$ angle, relative to the initial photon beam trajectory.

Spectra calculated for geometry "B", using MCNPX and the modified photonuclear data files are shown in Figure 5.6. Table 5.5 presents parameters describing the spectra shown in Figure 5.6, as well as those spectra that result from a photon beam incident as depicted in geometry "A". Similar to the simulation of the backscatter method, the intensities in Table 5.5 may be scaled to the intensities of resonant and non-resonant source photons and summed to determine the expected spectrum due to a given QM photon source distribution.

\subsubsection{Computed Photon Spectra using Quasi-monoenergetic Sources}

To demonstrate that beams comprised of resonant and non-resonant components would behave as a linear combination of their components, a series of simulations were conducted using Gaussian beam source energy profiles centered on the $2.431 \mathrm{MeV}{ }^{239} \mathrm{Pu}$ resonance, the irradiation geometry labeled "A" in Figure 5.5, and with ${ }^{239} \mathrm{Pu}$ content of $0 \%$ and 3.62 wt.\%. The source photon energies are determined by sampling the function given in equation 5.3 with $E_{C}=2.4315 \mathrm{MeV}$. Three simulated photon spectra due to $\sigma_{S}=100 \mathrm{eV}, 1 \mathrm{keV}$ and $10 \mathrm{keV}$ and $0 \%{ }^{239} \mathrm{Pu}$ are shown in Figure 5.7. In the simulations, the width of energy bins were assumed to be $3 \mathrm{keV}$, to demonstrate FWHM energy resolution of a HPGe detector at the resonance energy. At lower energies, the bin widths were increased to $24 \mathrm{keV}$ to improve statistics within the bins. Despite this, statistical uncertainties in the energy bins between $2 \mathrm{MeV}$ and $2.4 \mathrm{MeV}$ can reach $50 \%$, and the small visible

\footnotetext{
${ }^{1}$ Although a $1.5 \mathrm{~kg}{ }^{239} \mathrm{Pu}$ disc is technically feasible, smaller TD dimentions are more likely to be used at a facility. The radial dimension should be defined by the divergence of the photon source.
} 
Table 5.5 Parameters describing average spectra due to a single monoenergetic photon with $E=2431 \mathrm{keV}$ incident upon a spent fuel assembly that penetrate the fuel assembly, impinge upon the TD, and are backscattered toward a detector located $100 \mathrm{~cm}$ from the TD at an angle of $120^{\circ}$, relative to the initial beam direction. The energies of the monoenergetic photons were either considered to be that of the centroid energy of the $2431 \mathrm{keV}{ }^{239} \mathrm{Pu}$ resonance, or sufficiently distant from this energy that the resonance would have no impact on the transport of the photons. The geometry of the incident photon beam, relative to the assembly is depicted in Figure 5.5. $\frac{\Phi(E \leq 511)}{\Phi_{T}}$ indicates the fraction of the total calculated flux that are due to photons with energies $\leq 511 \mathrm{keV}$. $\Phi_{\mathrm{NRF}}$ is the expected flux of NRF $\gamma$ rays per source photon, $\Phi_{\mathrm{ES}}$ is the expected flux due to non-resonant elastic scatter of source-energy photons, and $\Phi_{T}$ is the expected total flux of photons due to monoenergetic, monodirectional photons normally incident upon a $1 \mathrm{~cm}$ diameter portion of a fuel assembly.

\begin{tabular}{ccccc}
\hline \hline $\begin{array}{c}\text { beam } \\
\text { geometry }\end{array}$ & $\frac{\Phi(E \leq 511)}{\Phi_{T}}$ & $\begin{array}{c}\Phi_{\mathrm{NRF}} \\
\left(1 / \mathrm{cm}^{2} / \mathrm{ph}\right)\end{array}$ & $\begin{array}{c}\Phi_{\mathrm{ES}} \\
\left(1 / \mathrm{cm}^{2} / \mathrm{ph}\right)\end{array}$ & $\begin{array}{c}\Phi_{T} \\
\left(1 / \mathrm{cm}^{2} / \mathrm{ph}\right)\end{array}$ \\
\hline$A_{\text {res }}$ & 0.41 & $3.6 \times 10^{-9}$ & $7.0 \times 10^{-13}$ & $8.2 \times 10^{-9}$ \\
$A_{n r}$ & 0.88 & 0 & $7.0 \times 10^{-13}$ & $3.3 \times 10^{-9}$ \\
$B_{\text {res }}$ & 0.37 & $1.5 \times 10^{-8}$ & $2.7 \times 10^{-12}$ & $3.3 \times 10^{-8}$ \\
$B_{n r}$ & 0.87 & 0 & $2.7 \times 10^{-12}$ & $1.2 \times 10^{-8}$ \\
\hline
\end{tabular}

peak at $1.93 \mathrm{MeV}$ is non-physical and due only to statistical fluctuations.

Using these simulations, the analytical attenuation model can be tested against MCNPX, the NRF signal intensity can be compared to the calculated background intensity, and the expected rates at which NRF and non-NRF $\gamma$ rays would be measured can be determined.

The intensity of the $2431 \mathrm{keV} \mathrm{NRF} \gamma$ ray compared to background was determined in two ways; for photon source distributions wider than the tally energy bin width, a Gaussian distribution was fit to the calculated peak due to non-resonant elastic scatter of source photons. The Gaussian shape can be seen for the $\sigma_{S}=10 \mathrm{keV}$ spectrum in Figure 5.7. For narrower source distributions, MCNPX calculations were also performed where the NRF process was turned off. Comparing spectra for identical runs with and without NRF indicates the relative intensity of the NRF $\gamma$ rays, relative to the non-resonant elastic scatter. The two techniques were found to provide similar estimates of the signal-to-background ratio. Figure 5.8 presents the calculated relative intensity of $\mathrm{NRF} \gamma$ rays at $2431 \mathrm{keV}$, relative to the calculated intensity of non-resonantly scattered photons that would be incident upon a detector.

As expected, the intensity of NRF $\gamma$ rays, relative to background increases when a larger fraction of the source photons are resonant-energy, as occurs with a narrower source. This effect becomes most pronounced when the width of the beam becomes less than the assumed detector resolution, at which point reducing $\sigma_{S}$ produces a smaller elastic scatter contribution. Using equation 2.23, we observe that the number of NRF counts needed to make a measurement of a given precision is twice as many for a $\sigma_{S}=20 \mathrm{keV}$ source than for a $\sigma_{S}=100 \mathrm{eV}$ source.

The resonant attenuation of photons predicted by these calculations is compared to the analytical predictions in the following way:

1. Two simulations, identical except in the ${ }^{239} \mathrm{Pu}$ content in the fuel assembly are compared;

2. Differences in non-resonant attenuation due to slight differences in $\mathrm{Pu}$ concentration in the fuel is corrected for by dividing each NRF peak by the intensity of the $511 \mathrm{keV}$ peak in the same spectrum; 

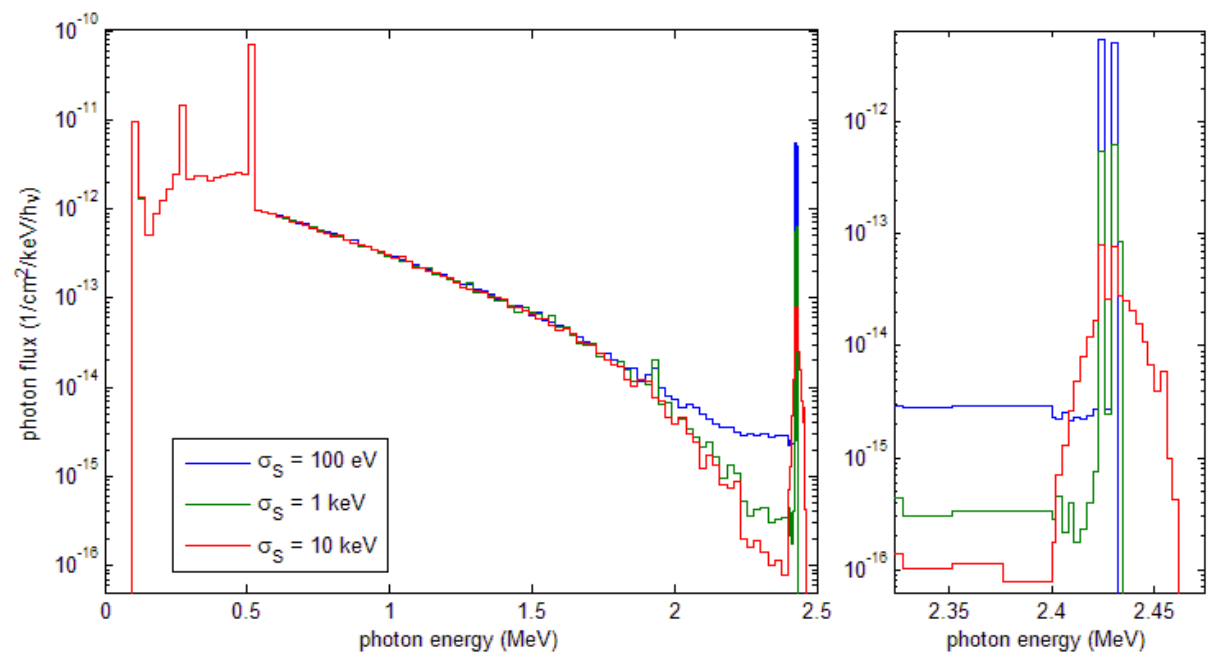

Figure 5.7 MCNPX-computed spectrum due to transmission measurement of fuel assembly containing $0 \%{ }^{239} \mathrm{Pu}$ using a Gaussian energy distribution of source photons centered at the $2.431 \mathrm{MeV}{ }^{239} \mathrm{Pu}$ resonance incident upon a spent fuel assembly as indicated by Geometry "A" in Figure 5.5. The right spectrum gives an expanded view of the region near the resonance energy.

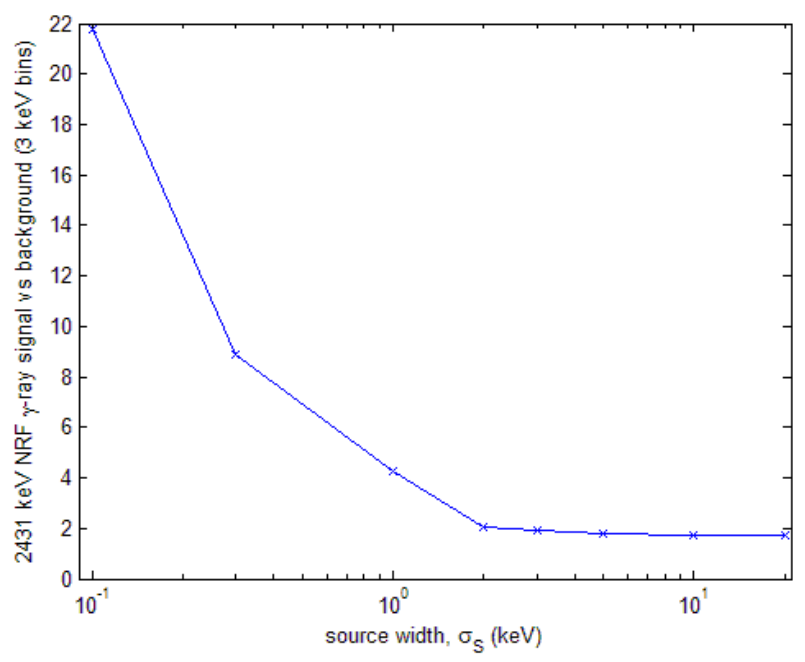

Figure 5.8 Calculated ratios of $2.431 \mathrm{MeV}$ NRF $\gamma$-ray flux at a detector location relative to background flux, $\xi$, within a $3 \mathrm{keV}$ energy bin. Statistical errors are less than $2 \%$. 
3. The normalized NRF peaks from the simulations of 3.62 wt. $\%{ }^{239} \mathrm{Pu}$ assemblies are divided by those from simulations with $0 \%{ }^{239} \mathrm{Pu}$ in the assemblies.

The resonant transmission is then given by

$$
\mathbb{A}=\frac{A_{3.62}^{\mathrm{NRF}}}{A_{0}^{\mathrm{NRF}}} \frac{A_{0}^{511}}{A_{3.62}^{511}}
$$

where $A_{\mathrm{wt} \%}^{p k}$ indicates the calculated area of the $511 \mathrm{keV}$ or the summed NRF peaks. These computed values are compared with the analytical attenation value in Figure 5.9. The analytical model appears capable of estimating the resonant attenaution of photons until $\sigma_{S}$ decreases below approximately $1 \mathrm{keV}$. At this point, the approximation that the source photon intensity is independent of energy is no longer valid. For truly monoenergetic photons, resonant attenuation of photons do not follow the analytical model, as written, but instead follow simple exponential attenuation,

$$
\mathbb{A}=\exp \left[-N \sigma_{\mathrm{NRF}}\left(E_{c}\right) x\right]
$$

where $\sigma_{\mathrm{NRF}}\left(E=E_{c}\right)$ is the value of the Doppler-broadened NRF cross section at the centroid energy. Using $8.65 \mathrm{~b}$, as the centroid value of the Doppler-broadened NRF cross section form implemented in MCNPX (see Appendix E), we obtain $\mathbb{A}=0.892$ for a monoenergetic photon source centered at the resonance energy incident upon a $3.62 \%{ }^{239} \mathrm{Pu}$ assembly. Using the routine described here, MCNPX produces $\mathbb{A}=0.890 \pm 0.007$, indicating that very narrow-band photon sources require additional detail in the analytical description. Also, the fact that the MCNPX values of $\mathbb{A}$ are slightly higher than the analytical value for $2 \mathrm{keV} \leq \sigma_{S} \leq 10 \mathrm{keV}$ may be due to notch refill. As indicated in Section 3.3.4, notch refill will reduce the intensity of the measured attenuation of that predicted by MCNPX (which properly accounts for notch refill) by approximately $5 \%$ i.e., $\mathbb{A}_{\text {meas }} \approx 1-.95\left(1-\mathbb{A}_{\text {model }}\right)$. Applying this correction results in excellent agreement between analytical and MCNPX $\mathbb{A}$ values.

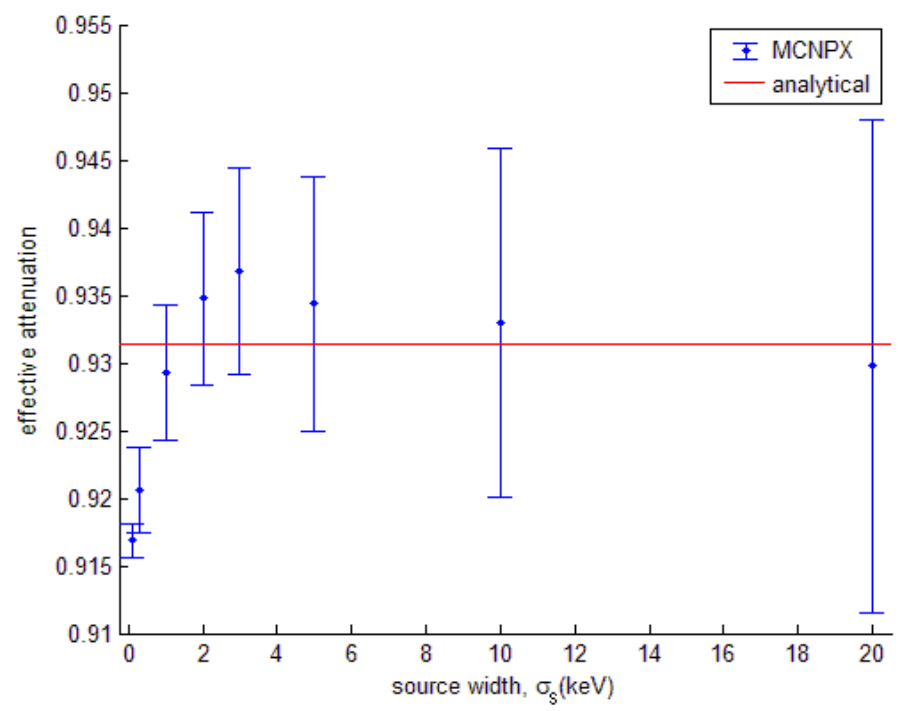

Figure 5.9 MCNPX-calculated reductions in the rate at which NRF $\gamma$ rays would be measured as a function of source photon beam energy width, $\sigma_{S}$. 


\subsubsection{Source Decomposition Method - Defining Relative Weights}

The values shown in Table 5.5 can be used to predict the total and NRF $\gamma$-ray fluxes shown in Figure 5.7. However, the simulated monoenergetic source photons need to be related to a source photon energy distribution. This is done by noting that the resonances are defined in MCNPX as spanning $8 \mathrm{eV}$ and then performing the following steps: We define the fractional flux of resonant energy source photons as

$$
\phi_{r e s}^{S}=\frac{8 \mathrm{eV}}{\sqrt{2 \pi \sigma_{S}^{2}}}
$$

and the flux due to non-resonant photons as

$$
\phi_{n r}^{S}=1-\psi \phi_{r e s}^{S}
$$

The term $\psi$ accounts for the fact that the resonant energy monoenergetic source photon evaluated the NRF cross section at the centroid energy, therefore to allow for a broad source energy distribution, we reduce the effect of resonant scatter by

$$
\psi=\frac{\int \sigma_{\mathrm{NRF}}(E) d E}{8 \mathrm{eV} \times \sigma_{\mathrm{NRF}}\left(E_{c}\right)}
$$

and $\psi=0.275$ for the $2431 \mathrm{keV}$ resonance of ${ }^{239} \mathrm{Pu}$.

Having determined the probability that a source photon from a Gaussian distribution may be considered resonant or non-resonant, we may determine the total photon flux due to a Gaussian source energy distribution, defined by the width, $\sigma_{S}$, as

$$
\Phi_{T}^{\sigma_{S}}=\phi_{n r}^{S} \Phi_{T}^{n r}+\psi \phi_{r e s}^{S} \Phi_{T}^{r e s}
$$

where $\Phi_{T}^{r e s}$ and $\Phi_{T}^{n r}$ are taken from Table 5.5. Likewise, the NRF $\gamma$-ray fluence is given by

$$
\Phi_{\mathrm{NRF}}^{\sigma_{S}}=\psi \phi_{\text {res }}^{S} \Phi_{\mathrm{NRF}}^{\text {res }}
$$

and entire spectra resulting from QM source photons can be decomposed into their contributions due to the transport of resonant and non-resonant energy photons.

We may then determine the total flux, the NRF $\gamma$ ray flux, and the signal-to-background ratio, $\xi$, for any combination of detector resolution and source width given a target that does not resonantly attenuate the photon source. For source widths broader than $\sim 300 \mathrm{eV}$, we may also accurately apply the analytical attenuation model to reduce the expected intensity of resonantenergy photons, which is expected to only be a few percent for ${ }^{239} \mathrm{Pu}$ in spent fuel.

Likewise, the values of $\Phi_{\mathrm{ES}}$ may be compared to those of $\Phi_{\mathrm{NRF}}$ in Table 5.5 to determine the expected signal-to-background ratio for measurement of an NRF peak, using the basis spectra. The intensity of elastically scattered source photons must be normalized by the fraction of source photons within the NRF energy bin. For the centroid bin, this is

$$
\phi_{\mathrm{ES}}=\operatorname{erf}\left(\frac{\Delta E_{b i n} / 2}{\sqrt{2 \sigma_{S}^{2}}}\right)
$$

where erf indicates the error function, $\operatorname{erf}(x)=2 \int_{0}^{x} \exp \left(-t^{2}\right) d t / \sqrt{\pi}$. Using equation 5.17 , we obtain similar signal-to-background ratios as were extracted from the series of simulations. 
Table 5.6 Transmission measurement parameters necessary to perform $1 \%$ measurment of ${ }^{239} \mathrm{Pu}$ in spent fuel assembly in Geometry "A", where $\rho_{\mathrm{Pu}}=0.72 \mathrm{~g} / \mathrm{cm}^{2}$, assuming HPGe detectors operate at $2 \times 10^{4} \mathrm{~Hz}$.

\begin{tabular}{ccccccc}
\hline \hline $\begin{array}{c}\sigma_{S} \\
(\mathrm{keV})\end{array}$ & $\begin{array}{c}\Phi_{\mathrm{NRF}}^{\sigma_{S}} \\
\left(1 / \mathrm{cm}^{2} / \mathrm{ph}\right)\end{array}$ & $\begin{array}{c}\Phi_{\mathrm{T}}^{\sigma_{S}} \\
\left(1 / \mathrm{cm}^{2} / \mathrm{ph}\right)\end{array}$ & $\xi$ & $\begin{array}{c}\mathrm{NRF} \\
\text { counts }\end{array}$ & $\begin{array}{c}t_{\text {meas }} \\
(\text { det.day })\end{array}$ & $\begin{array}{c}\text { intensity } \\
(1 / \mathrm{eV} / \mathrm{s})\end{array}$ \\
\hline 0.1 & $3.14 \times 10^{-11}$ & $3.34 \times 10^{-9}$ & 22.4 & $8.21 \times 10^{7}$ & 25.3 & $3.98 \times 10^{8}$ \\
0.3 & $1.05 \times 10^{-11}$ & $3.31 \times 10^{-9}$ & 7.47 & $9.55 \times 10^{7}$ & 87.6 & $1.34 \times 10^{8}$ \\
1 & $3.14 \times 10^{-12}$ & $3.30 \times 10^{-9}$ & 2.59 & $1.34 \times 10^{8}$ & 407 & $4.02 \times 10^{7}$ \\
2 & $1.57 \times 10^{-12}$ & $3.30 \times 10^{-9}$ & 2.05 & $1.49 \times 10^{8}$ & 907 & $2.01 \times 10^{7}$ \\
3 & $1.05 \times 10^{-12}$ & $3.30 \times 10^{-9}$ & 1.95 & $1.53 \times 10^{8}$ & $1.4 \times 10^{3}$ & $1.34 \times 10^{7}$ \\
5 & $6.27 \times 10^{-13}$ & $3.30 \times 10^{-9}$ & 1.90 & $1.55 \times 10^{8}$ & $2.4 \times 10^{3}$ & $8.06 \times 10^{6}$ \\
10 & $3.14 \times 10^{-13}$ & $3.30 \times 10^{-9}$ & 1.88 & $1.56 \times 10^{8}$ & $4.7 \times 10^{3}$ & $4.03 \times 10^{6}$ \\
20 & $1.57 \times 10^{-13}$ & $3.30 \times 10^{-9}$ & 1.87 & $1.56 \times 10^{8}$ & $9.5 \times 10^{3}$ & $2.01 \times 10^{6}$ \\
\hline
\end{tabular}

\subsubsection{Measurement Parameter Estimates Based on Source Energy Decomposition}

Using equation 3.29 and the exponential constant, $\alpha$ that relates areal densities to the effective resonant attenuation, $\left(=0.016 \mathrm{~g} / \mathrm{cm}^{2}\right.$ from Table 3.3 for the $2.431 \mathrm{MeV}$ resonance), we may derive an expression for the necessary number of counts, $C_{\mathrm{NRF}}$, to measure an areal density, $\rho_{\mathrm{Pu}}$ of ${ }^{239} \mathrm{Pu}$ in spent fuel to a precision given by the fraction uncertainty, $\epsilon / \rho_{\mathrm{Pu}}$ :

$$
C_{\mathrm{NRF}}=\frac{1+2 / \xi}{\left[\alpha \rho_{\mathrm{Pu}}\left(\epsilon / \rho_{\mathrm{Pu}}\right)\right]^{2}}
$$

\section{HPGe Detectors}

Equation 5.18 indicates that for $\epsilon / \rho_{\mathrm{Pu}}=1 \%$ and $\rho_{\mathrm{Pu}}=0.72 \mathrm{~g} / \mathrm{cm}^{2}$, this requires a total of $8.2 \times 10^{7}$ $\mathrm{NRF}$ counts for a $100 \mathrm{eV}$ wide source, which increases to $1.6 \times 10^{8} \mathrm{NRF}$ counts for sources wider than the detector resolution. In order for HPGe detectors to count scattered beam photons at a rate of $2 \times 10^{4} / \mathrm{s}$, the photon source would need to provide $10^{11}$ photons $/ \mathrm{s}$ on target, corresponding to beam intensities ranging from $4 \times 10^{8}-2 \times 10^{6} / \mathrm{eV} / \mathrm{s}$ for $100 \mathrm{eV}-20 \mathrm{keV}$ wide sources. These and many more parameters are summarized in Table 5.6 for Geometry "A", and Table 5.7 for Geometry "B"

The values shown in Tables 5.6 and 5.7 assume that the photon source intensity has the brightness stated in the last column. For larger peak intensity photon sources, thin filters could be used to reduce overall count rates without significantly reducing NRF count rates. For photon sources with lower brightness than those stated, the detection time fractionally increases. The detection times shown also assume a $1 \%$ precision measurement of ${ }^{239} \mathrm{Pu}$, less precise measurements reduce the counting time by the square of the fractional decrease.

Tables 5.6 and 5.7 illustrate the difficulty of performing a precise transmission measurement on ${ }^{239} \mathrm{Pu}$. The primary problems are that ${ }^{239} \mathrm{Pu}$ resonances are weak and the sources required are extremely intense. The LLNL-proposed QM source intensity of $10^{6}$ photons $/ \mathrm{s} / \mathrm{eV}$ with a width of a few $\mathrm{keV}$ would enable $1 \%$ measurments over $10^{4}$ detector days, or using a 40 detector array, 25 days; implying that a $5 \%$ measurement would take one day.

To illustrate the effects of resonance cross sections, another table, Table 5.8 analogous to Tables 5.6 and 5.7, is presented for a ${ }^{240} \mathrm{Pu}$ at $0.3 \mathrm{wt} . \%$ assuming that there exists a $80 \mathrm{eV} \cdot \mathrm{b}$ 
Table 5.7 Transmission measurement parameters necessary to perform $1 \%$ measurment of ${ }^{239} \mathrm{Pu}$ in spent fuel assembly in Geometry "B", where $\rho_{\mathrm{Pu}}=0.40 \mathrm{~g} / \mathrm{cm}^{2}$, assuming HPGe detectors operate at $2 \times 10^{4} \mathrm{~Hz}$.

\begin{tabular}{ccccccc}
\hline \hline $\begin{array}{c}\sigma_{S} \\
(\mathrm{keV})\end{array}$ & $\begin{array}{c}\Phi_{\mathrm{NRF}}^{\sigma_{S}} \\
\left(1 / \mathrm{cm}^{2} / \mathrm{ph}\right)\end{array}$ & $\begin{array}{c}\Phi_{\mathrm{T}}^{\sigma_{S}} \\
\left(1 / \mathrm{cm}^{2} / \mathrm{ph}\right)\end{array}$ & $\xi$ & $\begin{array}{c}\mathrm{NRF} \\
\text { counts }\end{array}$ & $\begin{array}{c}t_{\text {meas }} \\
(\text { det.day })\end{array}$ & $\begin{array}{c}\text { intensity } \\
(1 / \mathrm{eV} / \mathrm{s})\end{array}$ \\
\hline 0.1 & $1.34 \times 10^{-10}$ & $1.22 \times 10^{-8}$ & 24.8 & $2.64 \times 10^{8}$ & 69 & $1.09 \times 10^{8}$ \\
0.3 & $4.47 \times 10^{-11}$ & $1.21 \times 10^{-8}$ & 8.28 & $3.03 \times 10^{8}$ & 237 & $3.68 \times 10^{7}$ \\
1 & $1.34 \times 10^{-11}$ & $1.20 \times 10^{-8}$ & 2.87 & $4.15 \times 10^{8}$ & $1.08 \times 10^{3}$ & $1.11 \times 10^{7}$ \\
2 & $6.70 \times 10^{-12}$ & $1.20 \times 10^{-8}$ & 2.27 & $4.59 \times 10^{8}$ & $2.38 \times 10^{3}$ & $5.54 \times 10^{6}$ \\
3 & $4.47 \times 10^{-12}$ & $1.20 \times 10^{-8}$ & 2.16 & $4.70 \times 10^{8}$ & $3.65 \times 10^{3}$ & $3.69 \times 10^{6}$ \\
5 & $2.68 \times 10^{-12}$ & $1.20 \times 10^{-8}$ & 2.11 & $4.76 \times 10^{8}$ & $6.17 \times 10^{3}$ & $2.22 \times 10^{6}$ \\
10 & $1.34 \times 10^{-12}$ & $1.20 \times 10^{-8}$ & 2.08 & $4.79 \times 10^{8}$ & $1.24 \times 10^{4}$ & $1.11 \times 10^{6}$ \\
20 & $6.70 \times 10^{-13}$ & $1.20 \times 10^{-8}$ & 2.08 & $4.79 \times 10^{8}$ & $2.48 \times 10^{4}$ & $5.54 \times 10^{5}$ \\
\hline
\end{tabular}

Table 5.8 Transmission measurement parameters predicted to perform $1 \%$ measurment of ${ }^{240} \mathrm{Pu}$ in spent fuel assembly in Geometry "B", where $\rho_{\mathrm{Pu}}=0.24 \mathrm{~g} / \mathrm{cm}^{2}$, assuming HPGe detectors operate at $2 \times 10^{4} \mathrm{~Hz}$. The table assumes that ${ }^{240} \mathrm{Pu}$ has an $80 \mathrm{eV} \cdot \mathrm{b}$ resonance, although at this time the $\mathrm{NRF}$ response of ${ }^{240} \mathrm{Pu}$ has not been measrued.

\begin{tabular}{ccccccc}
\hline \hline $\begin{array}{c}\sigma_{S} \\
(\mathrm{keV})\end{array}$ & $\begin{array}{c}\Phi_{\mathrm{NRF}}^{\sigma_{S}} \\
\left(1 / \mathrm{cm}^{2} / \mathrm{ph}\right)\end{array}$ & $\begin{array}{c}\Phi_{\mathrm{T}}^{\sigma_{S}} \\
\left(1 / \mathrm{cm}^{2} / \mathrm{ph}\right)\end{array}$ & $\xi$ & $\begin{array}{c}\mathrm{NRF} \\
\text { counts }\end{array}$ & $\begin{array}{c}t_{\text {meas }} \\
(\text { det·day })\end{array}$ & $\begin{array}{c}\text { intensity } \\
(1 / \mathrm{eV} / \mathrm{s})\end{array}$ \\
\hline 0.1 & $5.65 \times 10^{-10}$ & $1.31 \times 10^{-8}$ & 105 & $3.90 \times 10^{7}$ & 2.62 & $1.01 \times 10^{8}$ \\
0.3 & $1.88 \times 10^{-10}$ & $1.24 \times 10^{-8}$ & 35 & $4.04 \times 10^{7}$ & 7.69 & $3.58 \times 10^{7}$ \\
1 & $5.65 \times 10^{-11}$ & $1.21 \times 10^{-8}$ & 12.1 & $4.46 \times 10^{7}$ & 27.7 & $1.10 \times 10^{7}$ \\
2 & $2.82 \times 10^{-11}$ & $1.21 \times 10^{-8}$ & 9.6 & $4.63 \times 10^{7}$ & 57.2 & $5.52 \times 10^{6}$ \\
3 & $1.88 \times 10^{-11}$ & $1.20 \times 10^{-8}$ & 9.1 & $4.67 \times 10^{7}$ & 86.4 & $3.68 \times 10^{6}$ \\
5 & $1.13 \times 10^{-11}$ & $1.20 \times 10^{-8}$ & 8.9 & $4.69 \times 10^{7}$ & 144 & $2.21 \times 10^{6}$ \\
10 & $5.65 \times 10^{-12}$ & $1.20 \times 10^{-8}$ & 8.8 & $4.70 \times 10^{7}$ & 289 & $1.11 \times 10^{6}$ \\
20 & $2.82 \times 10^{-12}$ & $1.20 \times 10^{-8}$ & 8.7 & $4.70 \times 10^{7}$ & 578 & $5.54 \times 10^{5}$ \\
\hline
\end{tabular}

resonance that could be measured. In this case, measurement duration is reduced by a factor of 25 - 50 compared to the ${ }^{239} \mathrm{Pu}$ measurement because $\alpha$, $\xi$, and $\Phi_{\mathrm{NRF}}^{\sigma_{S}}$ are increased by an amount proportional to the ratio of the NRF cross sections. Using a beam of the type proposed by LLNL would allow a $3 \%{ }^{240} \mathrm{Pu}$ measurement in one day with a $30 \mathrm{HPGe}$ detector array.

\section{High-resolution Scintillator Detectors}

Detectors capable of operating at higher count rates generally have worse energy resolution. However, when the width of the photon source is narrower than the energy resolution of the detector, detector resolution is not important. Given that $\mathrm{LaBr}_{3}$ detectors can operate at approximately 15 times the rate of HPGe detectors, increased photon beam intensities (relative to those indicated in Tables 5.6 - 5.8) can produce lower counting times. For beams with a narrow energy spread, the counting time reduction is directly proportional to the the beam intensity increase, whereas the improvement decreases with wider energy spread. Table 5.9 summarizes the times that a $1 \%$ measurement would take using a $\mathrm{LaBr}_{3}$ detector array versus a HPGe array. The $\mathrm{LaBr}_{3}$ detection times assume that the photon source is 15 times as intense as that stated in the previous tables 
Table 5.9 Comparing transmission measurement times for ${ }^{239} \mathrm{Pu}$ with detector arrays comprised of $\mathrm{LaBr}_{3}$ and HPGe detectors for different source widths, $\sigma_{S}$.

\begin{tabular}{lcccc}
\hline \hline $\begin{array}{l}\sigma_{S} \\
(\mathrm{keV})\end{array}$ & $\begin{array}{c}\text { Detection } \\
\text { Geometry }\end{array}$ & $\begin{array}{c}\mathrm{HPGe} \\
t_{\text {meas }} \\
\text { det·day) }\end{array}$ & $\begin{array}{c}\mathrm{LaBr}_{3} \\
t_{\text {meas }} \\
(\text { det·day })\end{array}$ & improvement \\
\hline 0.1 & $\mathrm{~A}$ & 25 & 1.7 & 15 \\
1.0 & $\mathrm{~A}$ & $4.1 \times 10^{2}$ & 29 & 14.1 \\
10 & $\mathrm{~A}$ & $4.7 \times 10^{3}$ & $1.5 \times 10^{2}$ & 3.1 \\
100 & $\mathrm{~A}$ & $4.7 \times 10^{4}$ & $7.4 \times 10^{3}$ & 1.6 \\
\hline 0.1 & $\mathrm{~B}$ & 69 & 4.6 & 15 \\
1.0 & $\mathrm{~B}$ & $1.1 \times 10^{3}$ & 76 & 14.1 \\
10 & $\mathrm{~B}$ & $1.2 \times 10^{4}$ & $3.8 \times 10^{3}$ & 3.3 \\
100 & $\mathrm{~B}$ & $1.2 \times 10^{5}$ & $7.7 \times 10^{4}$ & 1.6 \\
\hline
\end{tabular}

for the HPGe detector array.

Regardless of the detection geometry, faster detectors produce significant improvement for very intense narrow-band photon sources. When the beam width becomes comparable to the energy resolution of the detector, the detector type becomes less advantageous. Likewise, if photon beam intensities are not above those stated in Tables 5.6 - 5.8, faster detectors are not advantageous. However, if a photon beam could be made to produce $6 \times 10^{9}$ photons $/ \mathrm{eV} / \mathrm{s}$ on target, an array of one hundred $100 \%$ relative efficiency $\mathrm{LaBr}_{3}$ detectors could perform a $1 \%$ measurement of ${ }^{239} \mathrm{Pu}$ in spent fuel in about 25 minutes (1.7 detector-days/100 detectors). Such an array would correspond to subtending $7 \%$ of the backwards solid angle (or 0.43 steradians).

\section{Threshold Detectors}

Detectors that are only sensitive to photons above an energy threshold are useful for a transmission measurement if the intensity of source photons can be increased by a factor of over 100, compared to those that are presently proposed and a factor of $10^{5}$ over those under construction. This is because the processes of transmission through an assembly and subsequent backscatter in the TD sufficiently reduces the intensity of beam photons that reach energy-resolving detectors such that the detectors are not count-rate-limited. However, a system using threshold detectors would need to be well-calibrated and extremely stable in order that the observed reduced count rates can be attributed to resonant absorption of photons as they traverse the target, rather than system instability.

Using Table 5.5, we observe that approximately $1 / 2$ of the photon flux due to resonant, monoenergetic photons is due to NRF. As shown in Figure 5.6, above $2 \mathrm{MeV}$, the majority of the beam-induced backscattered flux is due to NRF and elastic scatter of beam photons. We may therefore consider the intensity of NRF $\gamma$ rays, relative to elastic scatter photons as an estimate of the value of $\xi$ that threshold detectors would experience. These values are shown in Table 5.10, as are the number of NRF counts that would be necessary to obtain $1 \%$ statistics. These numbers increase, relative to those for HPGe detectors, however, given that threshold detectors could perform in current mode, extremely bright beams could be used. Also indicated in Table 5.10 are the times (in detector-hours) and resulting count rates, $\dot{C}$, due to elastic scatter and NRF of source 
Table 5.10 Parameters for measuring ${ }^{239} \mathrm{Pu}$ in spent fuel assembly to $1 \%$ precision using QM photons with a beam brightness of $10^{11} / \mathrm{eV} / \mathrm{s}$ and a threshold detector of comparable volume to a $100 \%$ relative efficiency HPGe detector.

\begin{tabular}{ccccc}
\hline $\begin{array}{c}\sigma_{S} \\
(\mathrm{keV})\end{array}$ & $\xi$ & $\begin{array}{c}\text { NRF } \\
\text { counts }\end{array}$ & $\begin{array}{c}t_{\text {meas }} \\
\text { (det.hr })\end{array}$ & $\begin{array}{c}\dot{C} \\
(1 / \mathrm{s})\end{array}$ \\
\hline 0.1 & 22.4 & $8.2 \times 10^{7}$ & 4.8 & $4.9 \times 10^{3}$ \\
0.5 & 4.48 & $1.1 \times 10^{8}$ & 6.4 & $5.8 \times 10^{3}$ \\
1 & 2.24 & $2.4 \times 10^{8}$ & 8.4 & $6.8 \times 10^{3}$ \\
2.5 & 0.896 & $2.4 \times 10^{8}$ & 14 & $1.0 \times 10^{4}$ \\
10 & 0.22 & $7.5 \times 10^{8}$ & 44 & $2.6 \times 10^{4}$ \\
50 & 0.045 & $3.4 \times 10^{9}$ & 202 & $1.1 \times 10^{5}$ \\
100 & 0.024 & $6.8 \times 10^{9}$ & 400 & $2.2 \times 10^{5}$ \\
\hline
\end{tabular}

photons that would be detected assuming $10 \%$ of incident photons induce the threshold response, and that the beam brightness incident upon the fuel assembly is $10^{11} / \mathrm{eV} / \mathrm{s}$.

Extremely intense photon sources, coupled with one or more threshold type detectors could provide $1 \%$ precision measurement of ${ }^{239} \mathrm{Pu}$ in very short times. The use of narrower band sources is more important when using a threshold detector because of the inability to determine detected photon energies. 


\section{Conclusions and Future Work}

We have investigated the feasibility of applying NRF-based methods to the assay of SNF assemblies, i.e., to the measurement of $\mathrm{Pu}$ content in SNF and potential observation of fuel pin diversion. NRF measurement techniques are sensitive to individual isotopes and measure NRF rates that are directly proportional to the quantity of the measured isotope in a target. This direct correlation is a strong advantage of NRF measurement techniques in that diversion of a single fuel pin can in principle be detected. However, NRF signals are generally produced as only a small fraction of the total photon flux, which can cause signal-to-background difficulties and detector rate limitation issues. In light of these issues, the question of whether NRF measurement of SNF may be made with sufficient precision has been the primary subject of this study.

As an important tool we developed analytical models that allowed us to calculate detectable NRF signals for a variety of backscatter and transmission geometries. Extensive MCNPX modeling was performed to calculate and understand the scattered, non-resonant background, the notch refilling in transmission measurements, and to fully simulate NRF measurements on SNF assemblies. We found that MCNPX underestimated the non-resonant elastic scattering at backwards angles by multiple orders of magnitude mainly due to a flawed treatment of Rayleigh scattering in the code. We corrected this shortcoming and confirmed that the improved treatment of the elastic scattering leads to results in much better agreement with experimental data. Also added to the code was the ability to simulate NRF physics by adding known nuclear resonance data to the photonuclear datafiles so that NRF measurements could be fully modeled.

The main effort in this study was focused on analyzing and evaluating the application of NRF-based measurement methods to the determination of $\mathrm{Pu}$ isotopic content in SNF using the developed modeling tools. Because NRF signatures have only been measured for one isotope $\left({ }^{239} \mathrm{Pu}\right)$, the discussion has focused on this isotope, which is also the $\mathrm{Pu}$ isotope with the highest concentration in most SNF. Given the low concentrations of ${ }^{239} \mathrm{Pu}$ and its small integrated NRF cross sections, the main challenge in achieving precise and accurate measurements lies in accruing of sufficient counting statistics in a reasonable measurement time. This study focused on this challenge for ${ }^{239} \mathrm{Pu}$ and used ${ }^{238} \mathrm{U}$ to estimate responses of other isotopes while also acknowledging that systematic error sources would need to be understood, or at least controlled, in order to make sufficiently accurate measurements.

First, we assessed the potential capabilities of systems based on currently available instrumentation, i.e., systems that use bremsstrahlung photon sources and spectroscopic $\gamma$-ray detectors. Intense bremsstrahlung beams can be generated with Rhodotron accelerators that produce electron beams of up to $\sim 100 \mathrm{~mA}$ with several $\mathrm{MeV}$ energy. The backscattering method has three main limitations: a low signal-to-background ratio for the small $\mathrm{Pu}$ concentrations in SNF, a high background from the radioactive decay of the fission products in the spent fuel, and a strong dependence on depth of the intensity of the NRF signal. These difficulties render this approach not viable for the accurate measurement of $\mathrm{Pu}$ isotopes at the low concentrations found in SNF, using bremsstrahlung sources.

The transmission method provides two important advantages: first, the detectors can be shielded from $\gamma$ rays emitted from the fission products in the SNF, and second, the measure- 
ment sensitivity is not depth dependent. In this method the areal density of the $\mathrm{Pu}$ isotope is derived from the decrease of the NRF peaks in the measured spectrum. This decrease is on the order of $0.5 \%$ for the known ${ }^{239} \mathrm{Pu}$ resonances, implying very good counting statistics are needed to determine this quantity sufficiently well to precisely determine ${ }^{239} \mathrm{Pu}$ content. Due to the high flux of mostly lower-energy, scattered, non-resonant photons associated with bremsstrahlung beams, count rate-limited detectors need to be operated behind thick filters, which reduce already low $\mathrm{NRF}$ count rates further. As an example, we estimated that a measurement of $0.4 \%{ }^{239} \mathrm{Pu}$ with a few percent statistical error would take about 24 hours using a very intense bremsstrahlung source and a detector array that fills a significant fraction of the backwards hemisphere. The measurement for ${ }^{240} \mathrm{Pu}$ could be over ten times faster, if, as expected, this isotope has resonances with much larger cross sections. It is clear that sufficiently precise measurements of $\mathrm{Pu}$ isotope concentrations in SNF would require 10's to 100's of hours, a very intense bremsstrahlung source, and a very large array of fast detectors with energy resolutions near the best that scintillators can acheive, which could aslo be achieved with semiconductor type detectors.

Quasi-monoenergetic photon sources such as Laser Compton Scattering sources could potentially result in significantly different conclusions. Demonstration sources that produce low divergence, narrow beams of $2-2.5 \mathrm{MeV}$ photons with a 1 - $10 \mathrm{keV}$ energy spread are currently being constructed and construction of more intense sources is being proposed. Due to the narrow energy spread, the flux of lower-energy photons scattered into the detectors is dramatically reduced compared to bremsstrahlung sources. This results in systems that have higher resonant photon intensities and require less filtering without exceeding the detector count rate limitations, thus making shorter measurement times possible. We have estimated the times needed to reach $1 \%$ counting precision in a transmission measurement on a SNF assembly with $0.4 \%{ }^{239} \mathrm{Pu}$ content for a range of source parameters and detector options. As an example, for a photon source with a 1 $\mathrm{keV}$ energy spread, an intensity of $6 \times 10^{8} \mathrm{ph} / \mathrm{eV} \cdot \mathrm{s}$, and operating continuously or at $\mathrm{MHz}$ pulse rates, the measurement time would be 7 hours assuming an array of $100 \mathrm{LaBr}_{3}$ detectors $(100 \%$ relative efficiency) are used. Shorter times to obtain similar statistics would require sources with narrower energy spreads and higher intensities.

LCS sources of the type under development at LLNL are pulsed at $\mathrm{kHz}$ or lower rates. Threshold detectors, such as Cherenkov detectors, that can integrate the signal instead of detecting single photons are not rate-limited and thus would enable measurements with pulsed beams. Measurement times would be limited only by the intensity of the photon source. Interestingly, such threshold detectors would also make backscatter measurements with high-intensity pulsed photon sources possible. The detectors could be gated and not be sensitive in between short, sub-nanosecond pulses. This would reduce the background from the radioactivity of the SNF seen by the detectors by many orders of magnitude. In a best-case scenario a measurement with $1 \%$ statistical uncertainty could be accomplished with a $10^{8} \mathrm{ph} / \mathrm{eV} / \mathrm{s}$ photon source and an ideal Cherenkov detector array in $\sim 10 \mathrm{~min}$. While this indicates promise, neither the detectors nor the photon source presently exist. Ongoing developments of quasi-monoenergetic photon sources may reach $10^{6} \mathrm{ph} / \mathrm{eV} / \mathrm{s}$ and aerogel Cherenkov materials could potentially produce acceptable threshold characteristics.

It is important to note that ${ }^{240} \mathrm{Pu}$, as an even mass number isotope, likely has significantly larger resonances than ${ }^{239} \mathrm{Pu}$, which could result in measurement sensitivities that are more than ten times higher. The ability to precisely measure ${ }^{240} \mathrm{Pu}$ content may be useful to provide constraining information for other SNF measurements that determine fission rates per se, but require more 
information to relate that rate to total $\mathrm{Pu}$. Also, since ${ }^{240} \mathrm{Pu}$ content increases with total burn-up, measuring a lower than expected ${ }^{240} \mathrm{Pu}$ concentration in $\mathrm{SNF}$ could indicate weapons-grade $\mathrm{Pu}$ breeding.

We conclude from the results of this study that the potential of NRF-based methods for practical, direct, isotope-specific measurements cannot presently be realized and applied to the measurement of ${ }^{239} \mathrm{Pu}$ in SNF assemblies. Measurements with the required sensitivity and accuracy most likely require quasi-monoenergetic photon sources with intensities that are at least two orders of magnitude higher than those currently being designed or proposed. Such advancements may be possible and suitable sources may become available in the future, but substantial progress in electron accelerator and laser technologies is still needed.

\section{Future Work}

The first priority for assessing NRF measurements of $\mathrm{Pu}$ in SNF is to determine whether ${ }^{240} \mathrm{Pu}$ has sufficiently strong NRF resonances that its measurement could be accomplished with available technology in a practical system. Similar efforts to improve nuclear data for other isotopes that may prove important for safeguarding future nuclear fuel cycles is also of interest. The list of important actinides includes other long-lived $\mathrm{Pu}$ isotopes, ${ }^{233} \mathrm{U}$, and possibly other transuranics. For any isotope to be measured using NRF, the accuracy to which the measurement may be made will be limited by the accuracy and precision to which the resonance strength is known. Therefore more precise measurements of NRF resonance strengths should also be accomplished for all isotopes. Currently efforts are being made to perform initial measurements of ${ }^{240} \mathrm{Pu}$ and ${ }^{233} \mathrm{U}$ NRF responses and to improve measurements of the ${ }^{239} \mathrm{Pu}$ resonance strengths.

Other applications of NRF technology are also the subject of interest. Nuclear safeguards applications include measurement of uranium enrichment and actinide compositions in either traditional or non-traditional fuel types.

Since many actinide NRF responses are too weak to be useful in making make practical SNF measurements using available technology, additional effort should be devoted to the development of improved detectors and/or photon sources. Both fields are being actively pursued, although often for purposes different than applying NRF to nuclear safeguards. Of utmost interest would be the development of very fast, high-resolution $\gamma$-ray detectors, and the continued improvement of very intense LCS photon sources.

Along with technological and nuclear data improvements, the ability to model NRF experiments should be further improved. The non-resonant elastic scatter of photons is treated very approximately by ENDF, and resultingly is not well-modeled by most Monte Carlo radiation transport codes (MCNPX included). Improvement of NRES treatment as well as the variance reduction techniques used to simulate NRF will make future studies of any NRF measurement techniques more straight forward and accurate.

If and when $\gamma$-ray detector and/or photon source technology have sufficiently advanced that NRF measurements become significantly more practical, the design implementation and costs of such systems as well as more explicit treatment of potential systematic errors should be thoroughly addressed. These include beam and detector normalization and stability concerns, the detailed relationship between resonant attenuation and the content of the IOI in the target, and issues of notch refill. 
Appendices 


\section{A Transmission Nuclear Resonance Fluorescence Measurements of ${ }^{238} \mathbf{U}$ in Thick Targets}

\section{A.1 Measurement Summary}

This experiment was not part of the NGSI Spent Fuel Research but was supported by the MPACT campaign of the FCR\&D program of the Office of Nuclear Energy (US DOE) and by the Office of Proliferation Detection (US DOE, NA-221). However, this experimental work is closely related to the NGSI study of NRF for Spent Nuclear Fuel Assay reported here. It provides experimental validation of modeling performed for this study and demonstrates the transmission measurement method. Transmission nuclear resonance fluorescence measurements were made on targets consisting of $\mathrm{Pb}$ and depleted $\mathrm{U}$ with total areal densities near $86 \mathrm{~g} / \mathrm{cm}^{2}$. The ${ }^{238} \mathrm{U}$ content in the targets varied from 0 to $8.5 \%$ (atom fraction). The experiment demonstrates the capability of using transmission measurements as a non-destructive technique to identify and quantify the presence of an isotope in samples with thicknesses comparable to the average thickness of a nuclear fuel assembly. The experimental data also appear to demonstrate the process of notch refilling with a predictable intensity.

\section{A.2 Introduction and Experimental Goals}

In this section, we describe transmission NRF measurements of ${ }^{238} \mathrm{U}$ in thick targets using bremsstrahlung. The target dimensions were selected to have an areal density and attenuation properties similar to a nuclear fuel assembly so that the applicability of the transmission method as a nondestructive measurement technique for spent nuclear fuel could be tested. In this experiment, $\mathrm{Pb}$ was used as a surrogate for the $\mathrm{UO}_{2}$ matrix in spent fuel and the ${ }^{238} \mathrm{U}$ in depleted uranium (DU) was used as a surrogate for ${ }^{239} \mathrm{Pu}$ or any other minor actinide that would be measured in spent fuel. The amount of ${ }^{238} \mathrm{U}$ used in the experiment represents significantly higher concentrations than those of minor actinides in spent fuel. These amounts were selected to demonstrate the transmission attenuation effect in a timely manner using readily available radiation detectors and photon sources. A similar measurement has been made using thinner targets and a quasimonoenergetic photon source[42]. This measurement reported a null result for the observation of the notch refill phenomenon, whereas the data presented here indicate notch refill has occurred.

\section{A.3 Notch Refill}

The term notch refill is used to describe the process by which photons incident upon the assay geometry down-scatter to the energy of a resonance and subsequently interact in the TD. The process results in less observed resonant attenuation than would be predicted by consideration of simple exponential attenuation, and therefore neglect of the notch refill phenomenon would result in NRF transmission measurements that systematically under-predict the areal density of the measured isotope in the target. 
Photon interaction processes that can induce notch refill include incoherent scatter and bremsstrahlung emission from photoelectrons. The rate at which notch refill occurs is dependent upon the compositions of the assay target and transmission detection sheet, their respective positioning, the energy and strength of a resonance, and the photon spectrum used for the measurement.

Generally, notch refill cannot be directly measured because the energy resolution required for such a measurement is approximately $1 \mathrm{eV}$ for a resonance at $2 \mathrm{MeV}$. However, the processes that result in notch refill are readily computed using Monte Carlo radiation transport computer codes such as MCNPX[5]. Comparing MCNPX calculations with models that exclude notch refill gives an estimate of the notch refill intensity. As discussed in Section A.6, the intensity of the notch refill corrections appears to be consistent with the measured data.

Transmission experiments have previously neglected notch refilling because target areal densities were generally significantly smaller[3, 42, 43]. Thinner target thicknesses are better for measurement of resonant state parameters, but the targets used in this experiment were intended to be representative of nuclear fuel assemblies. These thicker targets cause notch refill to be no longer negligible, and the positioning of $\mathrm{DU}$ and $\mathrm{Pb}$ in the targets was further selected to maximize the notch refill effect by placement of the $\mathrm{Pb}$, which induced scatter but no resonant absorption, downstream of the absorbing DU.

\section{A.4 Experimental Setup}

The experiment was conducted at the High Voltage Research Laboratory at the Massachusetts Institute of Technology. Electrons accelerated to $2.60 \pm 0.03 \mathrm{MeV}$, where the indicated uncertainty indicates beam stability, by a Van de Graaff accelerator were transported through a beamline, bent 90 degrees, and entered the experimental geometry as shown in Figure A.1. The converter target consisted of a $102 \mu \mathrm{m}$ thick $\mathrm{Au}$ layer on a $1 \mathrm{~cm}$ thick water-cooled $\mathrm{Cu}$ backing. The electron current incident upon the converter was approximately $65 \mu \mathrm{A}$ throughout the experiment. The bremsstrahlung emitted from the converter target was shaped by a $20 \mathrm{~cm}$ thick $\mathrm{Pb}$ collimator. The diameter of the collimator opening was $1 \mathrm{~cm}$ on the bremsstrahlung converter side, and increased to $2.5 \mathrm{~cm}$, resulting in a $3.6^{\circ}$ opening from the beam center, and a maximum angular acceptance of $5^{\circ}$.

Figure A.1, shows the important components: the bremsstrahlung converter and collimator, the assay target, the transmission detection sheet, the HPGe detectors and the Pb shielding. The assay target, a combination of $\mathrm{DU}$ and $\mathrm{Pb}$, was located directly downstream of the collimator opening. The areal densities of $\mathrm{DU}$ and $\mathrm{Pb}$ used in the assay targets are summarized in Table A.1. The DU was placed up-stream of the $\mathrm{Pb}$ in the beam, setting up the most probable geometry for notch refill.

The transmission detection sheet was placed $142 \mathrm{~cm}$ downstream of the collimator opening and was not moved throughout the experiment. It consisted of five $20.32 \mathrm{~cm} \mathrm{x} 20.32 \mathrm{~cm}$ DU sheets that were nominally $0.8 \mathrm{~mm}$ thick. Each sheet was contained in two plastic bags. The total mass of the five plates plus the containment bags was measured to be $3341.7 \pm 3.3 \mathrm{~g}$, of which 3226.7 $\pm 28 \mathrm{~g}$ are attributed to the DU. Behind the DU plates, $82.3 \pm 0.5 \mathrm{~g}$ of $\mathrm{Mn}$ and $185.1 \pm 0.8 \mathrm{~g}$ of $99.52 \%$ enriched ${ }^{11} \mathrm{~B}$ were also positioned in the beam to serve as auxiliary flux monitors.

Two pairs of approximately 100\% relative efficiency HPGe detectors were positioned $27 \mathrm{~cm}$ from the intersection of the beam center and the transmission detection sheet, at an angle of $118^{\circ}$ relative to the centroid beam direction. Actual detector acceptances were distributed around the 


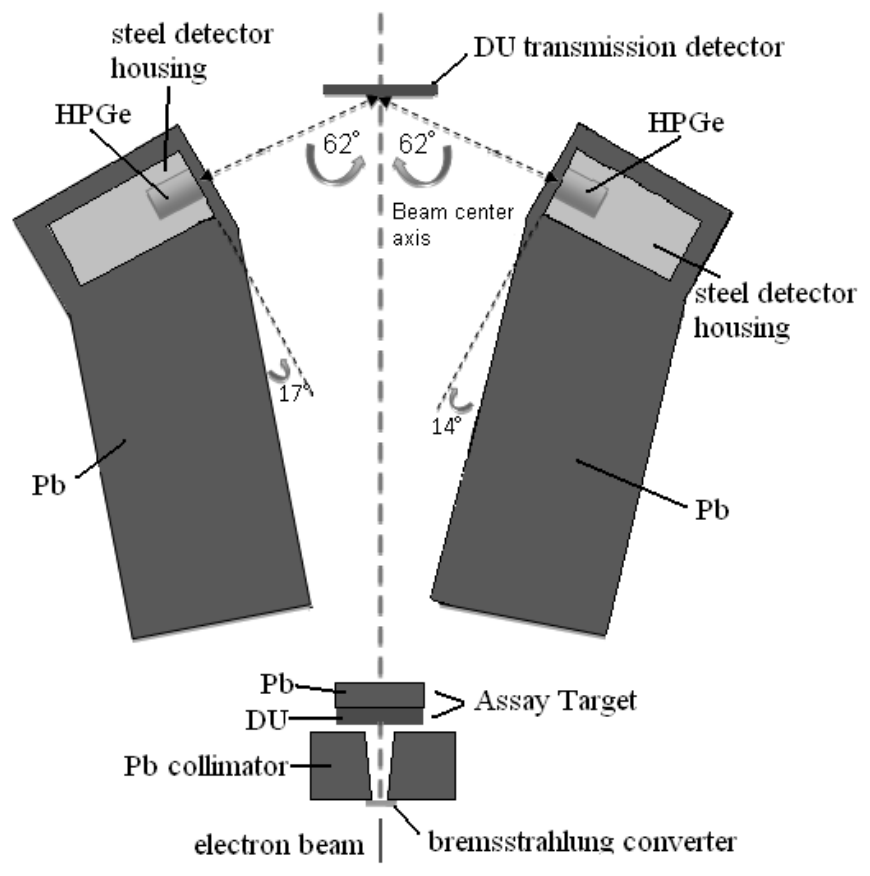

Figure A.1 Schematic view of experimental setup. DU thicknesses have been exaggerated for visibility.

Table A.1 Assay target compositions. Pb density assumed to be $11.34 \mathrm{~g} / \mathrm{cm}^{3}$, DU sheets were assumed to be $99.799 \%{ }^{238} \mathrm{U}$ by mass. Uncertainties in DU masses are approximately $1 \%$.

\begin{tabular}{ccccc}
\hline \hline run & $\begin{array}{c}\rho x_{\mathrm{DU}} \\
\left(\mathrm{g} / \mathrm{cm}^{2}\right)\end{array}$ & $\begin{array}{c}\rho x_{\mathrm{Pb}} \\
\left(\mathrm{g} / \mathrm{cm}^{2}\right)\end{array}$ & $\begin{array}{c}\text { total } \rho x \\
\left(\mathrm{~g} / \mathrm{cm}^{2}\right)\end{array}$ & $\begin{array}{c}{ }^{238} \mathrm{U} \\
\text { atom } \%\end{array}$ \\
\hline 1 & 0 & 86.26 & 86.26 & 0 \\
2 & 8.47 & 79.85 & 87.86 & 8.48 \\
3 & 1.69 & 85.20 & 86.80 & 1.69 \\
4 & 3.34 & 83.37 & 86.54 & 3.37 \\
\hline
\end{tabular}


Table A.2 $\gamma$-ray lines identified in overnight background spectra used to calibrate energy spectra[44].

\begin{tabular}{cc}
\hline \hline$E_{\text {line }}(\mathrm{keV})$ & Isotope \\
\hline 766.36 & ${ }^{234 \mathrm{~m}} \mathrm{~Pa}$ \\
1001.03 & ${ }^{234 \mathrm{~m}} \mathrm{~Pa}$ \\
1193.77 & ${ }^{234 \mathrm{~m}} \mathrm{~Pa}$ \\
1460.83 & ${ }^{40} \mathrm{~K}$ \\
1737.73 & ${ }^{234 \mathrm{~m}} \mathrm{~Pa}$ \\
1911.17 & ${ }^{234 \mathrm{~m}} \mathrm{~Pa}$ \\
2204.21 & ${ }^{214} \mathrm{Bi}$ \\
2614.49 & ${ }^{208} \mathrm{Tl}$ \\
\hline
\end{tabular}

centroid angle with an approximate $10^{\circ}$ width. The detectors were contained in steel housings that were stacked in pairs, resulting in one detector being centered $4.5 \mathrm{~cm}$ above the beam center and the other centered $7 \mathrm{~cm}$ below the beam center.

$\mathrm{Pb}$ brick walls with minimum thicknesses of $80 \mathrm{~cm}$ were constructed to shield the radiation detectors from the bremsstrahlung converter and assay target. The detectors were also shielded with $5 \mathrm{~cm}$ of $\mathrm{Pb}$ in the down-stream direction, $10 \mathrm{~cm}$ of $\mathrm{Pb}$ below and behind the detectors, $17 \mathrm{~cm}$ of $\mathrm{Pb}$ above the detectors, and with a $1.27 \mathrm{~cm}$ thick $\mathrm{Pb}$ filter facing the transmission detection sheet. The filter reduces the intensity of low-energy photons emitted from the transmission detection sheet due to Compton scatter and other processes.

Each target was irradiated for approximately 7 hour measurements. The electron beam current was nominally $65 \mu \mathrm{A}$ for each irradiation. The detectors were operated with Ortec DSPEC Pro $^{\text {TM }}$ digital $\gamma$-ray spectrometers. The integrated pulse amplitudes were read and stored using MAESTRO-32 and a personal computer. In this configuration, the ADC rates were approximately $10^{4}$ counts/s.

\section{A.5 Data Analysis}

Spectra were collected during four day long irradiation shifts and background spectra, with no electron beam incident upon the bremsstrahlung converter, were collected overnight. The radioactivity of the DU and ambient ${ }^{40} \mathrm{~K}$ provided lines for energy-calibrating the detectors. The $\gamma$-ray energies used for energy calibration are shown in Table A.2. After calibrating the four detectors, their spectra were re-binned to a common energy grid and summed to provide a single spectrum for each target. An example of spectral summing is provided in Figure A.2. After summing, peaks that were present in spectra taken during irradiations but not in background spectra were attributed to NRF.

To correct for varying beam intensity and the differing attenuation lengths of the targets, the rates at which $511 \mathrm{keV} \gamma$ rays were measured in the detectors were compared. To demonstrate the accuracy of this normalization, a series of calculations were performed using MCNPX. The calculations simulated a bremsstrahlung spectrum transported through the four different targets. The photon spectra that reached the transmission detection sheet after emission from each of the assay targets is defined as $\Phi^{i}(E)$, where the superscript indicates the target used in the run, whose number is indicated in Table A.1. Each of these computed spectra were also convolved with the cross section for pair production in $\mathrm{U}[25]$ to provide a quantity proportional to the rate at which 


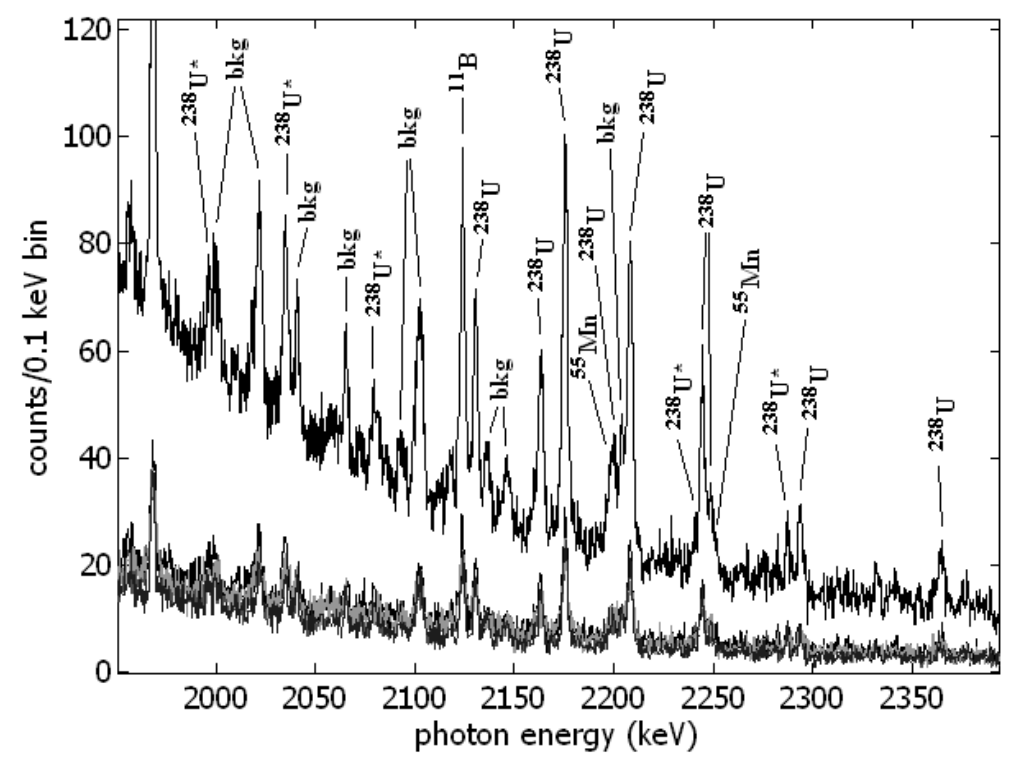

Figure A.2 Spectra from all four detectors collected during run 1. The lower spectra are from the four different detectors before summing. Summing of these spectra produce the upper spectrum, which includes labeling to indicate the source of identified peaks. 'bkg' indicates the peak is present in the radioactive background spectrum, ${ }^{238} \mathrm{U}$ ' indicates the peak is due to a known ${ }^{238} \mathrm{U}$ NRF resonance, and ${ }^{238} \mathrm{U}^{*}$ ' indicates the peak is suspected to be due to ${ }^{238} \mathrm{U}$ NRF.

pair production occurred in the transmission detection sheet,

$$
R_{\mathrm{PP}}^{i}=\int \Phi^{i}(E) \sigma_{\mathrm{PP}}(E) d E
$$

Computed photon spectra, $\Phi^{i}(E)$, were then normalized by dividing each spectrum by $R_{\mathrm{PP}}^{i}$, and compared. The quantity,

$$
\Phi_{N}^{i}(E)=\frac{\Phi^{i}(E) / R_{\mathrm{PP}}^{i}}{\Phi^{1}(E) / R_{\mathrm{PP}}^{1}}
$$

is shown in Figure A.3 for the three targets containing DU. If the simulations indicated that normalization by measured pair production rates were perfect, the functions, $\Phi_{N}^{i}(E)$ would be unity at all energies. The calculations indicate that below approximately $1.8 \mathrm{MeV}$, normalizing spectra incident upon the transmission detection sheet by the intensity of pair production would result in large errors. However, the normalization needs only to be accurate in the range of the NRF resonances: $2.1-2.5 \mathrm{MeV}$. In this range the calculations indicate that by using the measured $511 \mathrm{keV}$ peak to normalize the intensity of photons reaching the transmission detection sheet after penetrating through differing targets, should incur less than a $1.5 \%$ systematic error ${ }^{1}$. The error bars in Figure A.3 indicate the uncertainty of the MCNPX calculation, and do not include the systematic error due to the linear, rather than logarithmic, interpolation of atomic scattering functions that is performed in MCNPX[45]. The saw-tooth shape of the inset in Figure A.3 can be attributed to these interpolation errors, and therefore the actual systematic error due to this normalization technique may be closer to $0.5 \%$.

\footnotetext{
${ }^{1}$ Experience has indicated that the normalization routine is best suited for comparisons between similar targets. Because of this, we anticipate that it could prove useful for beam normalization when making relative $\mathrm{Pu}$ concentration measurements in SNF compared to a reference assembly.
} 


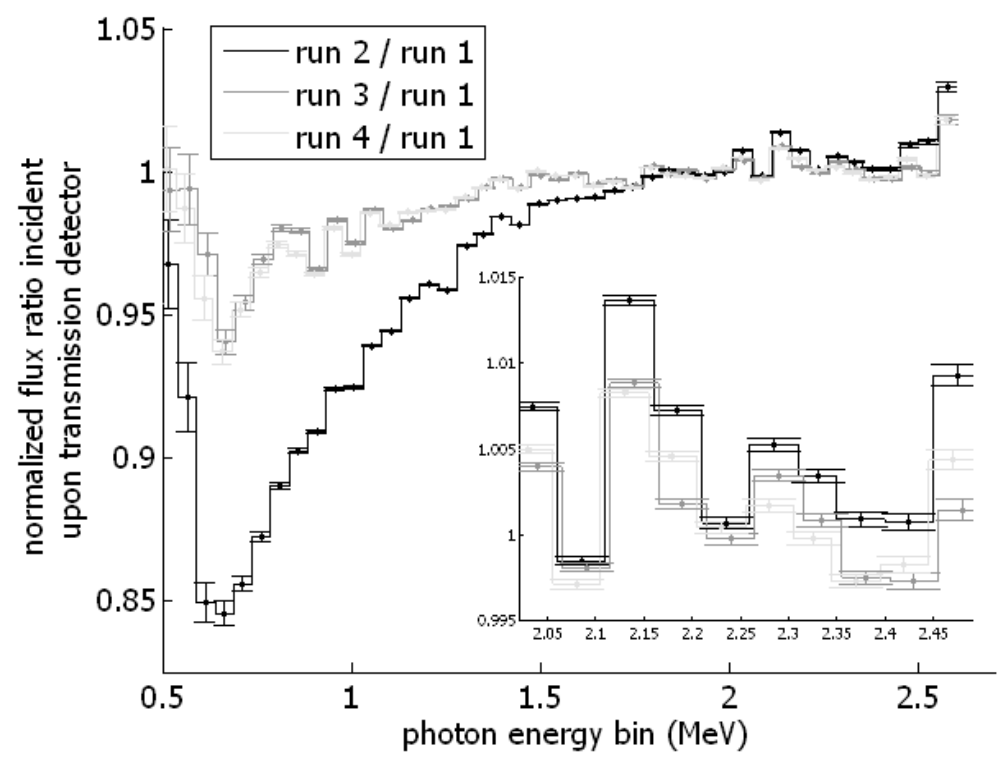

Figure A.3 Calculated values of $\Phi_{N}^{i}(E)$, given by Equation A.2 for the measurement of the targets containing $\mathrm{DU}, \mathrm{i}=2,3$, and 4 .

${ }^{238} \mathrm{U}$ NRF peaks identified in runs 2 - 4 were fit, integrated, and compared to corresponding peak intensities from run 1. The comparison of peak intensities is expressed as

$$
\mathbb{A}_{E_{p k}}^{i}=\frac{A_{E_{p k}}^{i}}{A_{E_{p k}}^{1}} \frac{A_{511}^{1}}{A_{511}^{i}}
$$

where $\mathbb{A}_{E_{p k}}^{i}$ is the relative intensity of the peak at $E=E_{p k}$ for run $i$, relative to run $1 . A_{E_{p k}}^{i}$ corresponds to the fit area of that peak. Each resonance yielded two observable peaks, due to de-excitation of the NRF level directly to the ground level, and also due to de-excitation via the first-excited state. The two values of $\mathbb{A}_{E_{p k}}^{i}$ for each level were averaged, resulting in a single value of $\mathbb{A}_{\text {level }}^{i}$ for each NRF level, measured in each of runs 2 - 4. These values are shown in the last columns of Table A.3.

\section{A.6 Areal Density Measurement}

The areal density of an isotope in an irradiated target is related to the attenuation of resonant energy photons, as observed by a reduction in the rate that radiation detectors measure NRF $\gamma$ rays emitted from the transmission detection sheet. For each ${ }^{238} \mathrm{U}$ resonance, a function was derived that relates the rate at which NRF $\gamma$ rays are emitted from the transmission detection sheet to the areal density of ${ }^{238} \mathrm{U}$ in the assay target. This function, for a target containing the measured isotope at an areal density, $\rho x$, is given by

$$
\mathbb{A}_{\text {model }}(\rho)=C_{\text {notch }} \frac{\int \lambda\left(t_{\mathrm{TD}}, E\right) \exp \left[-\sigma_{D}(E) \rho x\right] \sigma_{D}(E) d E}{\int \lambda\left(t_{\mathrm{TD}}, E\right) \sigma_{D}(E) d E}
$$


Table A.3 Predicted and measured values of $\mathbb{A}_{\text {level }}^{i}$ with statistical errors. The levels at 2467 and $2080 \mathrm{keV}$ have not been previously reported and therefore no prediction of $\mathbb{A}_{\text {level }}^{i}$ was made. Predicted values of $\mathbb{A}_{\text {level }}^{i}$ are obtained from evaluation of Equation A.4 using the values of $\Gamma$ and $\Gamma_{0}$ reported in Heil et al.[27] and that $C_{\text {notch }}=1$, column $\mathrm{C}$ uses values of $\Gamma$ and $\Gamma_{0}$ from ENSDF, columns B and D use the same values as A and C, respectively, but use the best-estimate of $C_{\text {notch }}$ shown in Figure A.4.

\begin{tabular}{|c|c|c|c|c|c|c|c|c|}
\hline \multirow{2}{*}{\multicolumn{2}{|c|}{$\begin{array}{r}E_{\text {level }} \\
(\mathrm{keV})\end{array}$}} & \multicolumn{5}{|c|}{ predicted attenuation } & \multirow{2}{*}{\multicolumn{2}{|c|}{$\begin{array}{c}\text { measured } \\
\text { attenuation }\end{array}$}} \\
\hline & & A & B & $\mathrm{C}$ & $\mathrm{D}$ & uncertainty & & \\
\hline \multirow{8}{*}{$\underset{J}{J}$} & 2175.6 & 0.54 & 0.57 & 0.55 & 0.58 & \pm 0.018 & 0.58 & \pm 0.05 \\
\hline & 2208.2 & 0.55 & 0.58 & 0.57 & 0.59 & \pm 0.019 & 0.62 & \pm 0.06 \\
\hline & 2246.7 & 0.71 & 0.73 & 0.72 & 0.74 & \pm 0.016 & 0.72 & \pm 0.09 \\
\hline & 2293.8 & 0.87 & 0.87 & 0.87 & 0.88 & \pm 0.014 & 0.94 & \pm 0.16 \\
\hline & 2409.7 & 0.78 & 0.80 & 0.79 & 0.80 & \pm 0.013 & 0.84 & \pm 0.16 \\
\hline & 2467.4 & 0.76 & 0.78 & 0.77 & 0.79 & \pm 0.016 & 0.65 & \pm 0.18 \\
\hline & 2080.0 & & & & & & 0.87 & \pm 0.15 \\
\hline & 2287.4 & & & & & & 0.67 & \pm 0.27 \\
\hline \multirow{8}{*}{$\begin{array}{l}N \\
\Xi \\
J\end{array}$} & 2175.6 & 0.88 & 0.89 & 0.88 & 0.89 & \pm 0.006 & 0.92 & \pm 0.06 \\
\hline & 2208.2 & 0.88 & 0.89 & 0.89 & 0.90 & \pm 0.006 & 0.93 & \pm 0.07 \\
\hline & 2246.7 & 0.93 & 0.94 & 0.94 & 0.94 & \pm 0.004 & 0.84 & \pm 0.09 \\
\hline & 2293.8 & 0.97 & 0.97 & 0.97 & 0.97 & \pm 0.003 & 0.99 & \pm 0.16 \\
\hline & 2409.7 & 0.95 & 0.95 & 0.95 & 0.96 & \pm 0.003 & 1.00 & \pm 0.16 \\
\hline & 2467.4 & 0.95 & 0.95 & 0.95 & 0.95 & \pm 0.004 & 1.02 & \pm 0.21 \\
\hline & 2080.0 & & & & & & 0.99 & \pm 0.18 \\
\hline & 2287.4 & & & & & & 0.75 & \pm 0.27 \\
\hline \multirow{9}{*}{$\sum_{j=1}^{\infty}$} & 2175.6 & 0.77 & 0.79 & 0.78 & 0.79 & \pm 0.011 & 0.82 & \pm 0.06 \\
\hline & 2208.2 & 0.78 & 0.80 & 0.79 & 0.81 & \pm 0.011 & 0.80 & \pm 0.07 \\
\hline & 2246.7 & 0.87 & 0.88 & 0.88 & 0.89 & \pm 0.008 & 0.89 & \pm 0.10 \\
\hline & 2293.8 & 0.94 & 0.95 & 0.95 & 0.95 & \pm 0.006 & 0.84 & \pm 0.15 \\
\hline & 2409.7 & 0.91 & 0.91 & 0.91 & 0.92 & \pm 0.006 & 1.00 & \pm 0.17 \\
\hline & 2467.4 & 0.90 & 0.90 & 0.90 & 0.91 & \pm 0.007 & 1.04 & \pm 0.22 \\
\hline & 2080.0 & & & & & & 0.90 & \pm 0.16 \\
\hline & 2287.4 & & & & & & 0.59 & \pm 0.37 \\
\hline & $\chi^{2}$ & 6.84 & 4.79 & 5.72 & 4.34 & & & \\
\hline
\end{tabular}


where $\lambda\left(t_{\mathrm{TD}}, E\right)$ is the effective thickness a photon of energy, E, traverses in a transmission detection sheet having a thickness, $t_{\mathrm{TD}}$, and atom density, $N_{\mathrm{TD}}$, given by

$$
\lambda\left(t_{\mathrm{TD}}, E\right)=\frac{1-\exp \left[-\left(\alpha+N_{\mathrm{TD}} \sigma_{D}(E)\right) t_{\mathrm{TD}}\right]}{\alpha+N_{\mathrm{TD}} \sigma_{D}(E)}
$$

and $\alpha=\mu_{n r}[1+1 / \cos (\theta)]$ and $\theta \approx 62^{\circ}$ for the experimental geometry. The parameter $\mu_{n r}$ contains the attenuation coefficients for all non-resonant photon scattering events, and has been retrieved from the XCOM database[25].

The quantity, $C_{\text {notch }}$, accounts for notch refill. To obtain values of $C_{\text {notch }}$, simulations of the experimental geometry were conducted using MCNPX and the new NRF data library[46] to obtain $\mathbb{A}(\rho)$. These values were compared to analytical evaluations of $\mathbb{A}_{\text {model }} / C_{\text {notch }}$, giving $C_{\text {notch. Values }}$ of $C_{\text {notch }}$ are shown in Figure A.4 for the 2176,2209 and $2245 \mathrm{keV}{ }^{238} \mathrm{U}$ resonances. The notch refill process is most intense for stronger and lower-energy resonances, and is more important for thicker targets. As seen in Figure A.4, the effect of notch refill is estimated not to exceed $5 \%$ for this experiment.

Columns A - D in Table A.3 present calculated values of $\mathbb{A}_{\text {model }}(\rho)$ for four different treatments of Equation A.4. Values in columns A and B assume that the resonance parameters as reported by Heil et al.[27] are correct, whereas the values in columns $\mathrm{C}$ and $\mathrm{D}$ were obtained using the resonance parameters described in ENSDF. Columns $\mathrm{A}$ and $\mathrm{C}$ assume that $C_{\text {notch }}=1$, and columns

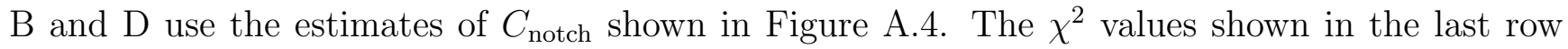
of Table A.3 are determined by comparing the 18 measured $\mathbb{A}_{\text {level }}^{i}$ values to those predicted in columns A - D. Only the statistical uncertainties of each measurement was used to calculate the reported $\chi^{2}$ values $^{2}$. Use of the notch-refill correction and the ENSDF evaluation of the resonance parameters (column D) provided the best agreement between data and prediction and therefore this treatment is used to determine ${ }^{238} \mathrm{U}$ areal densities in the targets as measured by transmission NRF. The predicted attenuation values shown in column B, using the Heil et al. interpretation of the resonance data with the notch refill correction, demonstrates better agreement to the data than either predicted attenuation that omitted the notch refill correction.

Using the values, $C_{\text {notch }}(\rho)$ shown in Figure A.4 and the resonance parameters from ENSDF, Equation A.4 is evaluated and values of $\mathbb{A}_{\text {model }}(\rho)$ corresponding to values in column D of Table A.3 are shown in Figure A.5. The dotted lines indicate errors due to the uncertainty of $\Gamma_{0}$ from the previous measurement of the ${ }^{238} \mathrm{U}$ resonances.

For each measured value of $\mathbb{A}_{\text {level }}^{i}$ shown in Table A.3, $\mathbb{A}_{\text {model }}(\rho)$ was inverted to obtain an areal density of ${ }^{238} \mathrm{U}$ that would produce the corresponding attenuation. These values were combined for the six known resonances by calculating the weighted average of each estimated areal density. The weighted averages are shown in Table A.4 with experimental statistical errors and systematic errors due to uncertainties in the strengths of the ${ }^{238} \mathrm{U}$ resonances.

\section{A.7 Conclusion}

The determination of ${ }^{238} \mathrm{U}$ areal densities ranging between 1.7 and $8.5 \mathrm{~g} / \mathrm{cm}^{2}$ in an approximately $86 \mathrm{~g} / \mathrm{cm}^{2}$ target by observation of attenuation of resonant-energy photons has been accomplished.

\footnotetext{
${ }^{2}$ Inclusion of the statistical uncertainties reported by Heil et al. into the $\chi^{2}$ calculation does not significantly alter the results.
} 


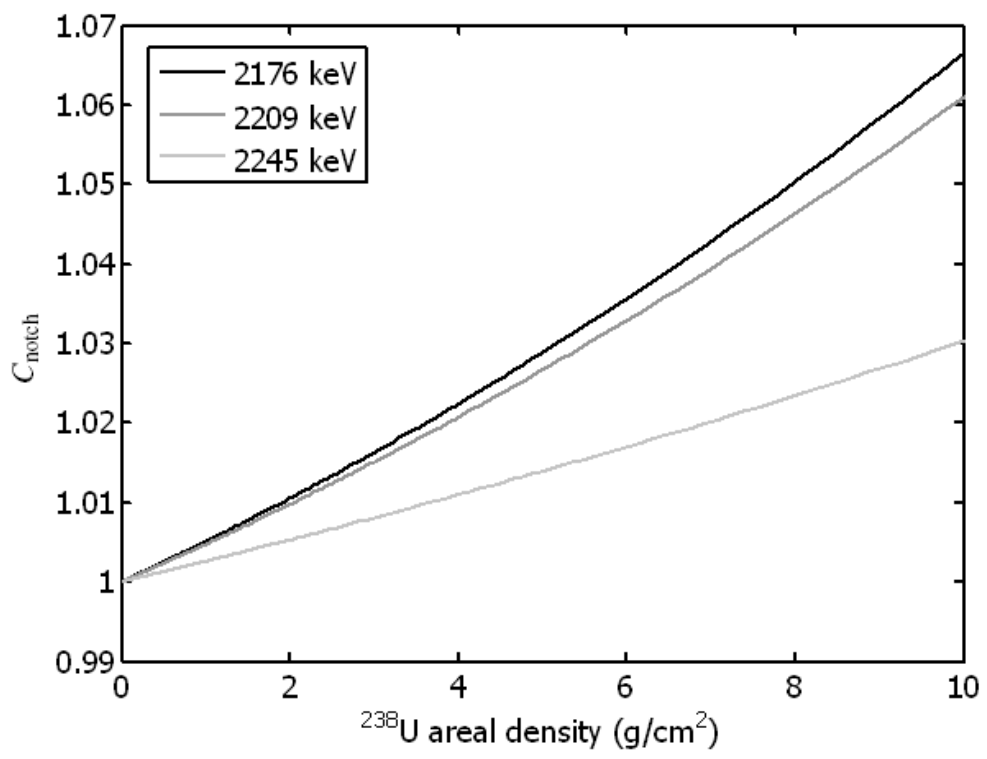

Figure A.4 Estimated intensities of notch refilling for three ${ }^{238} \mathrm{U}$ resonances indicated by their centroid energy.

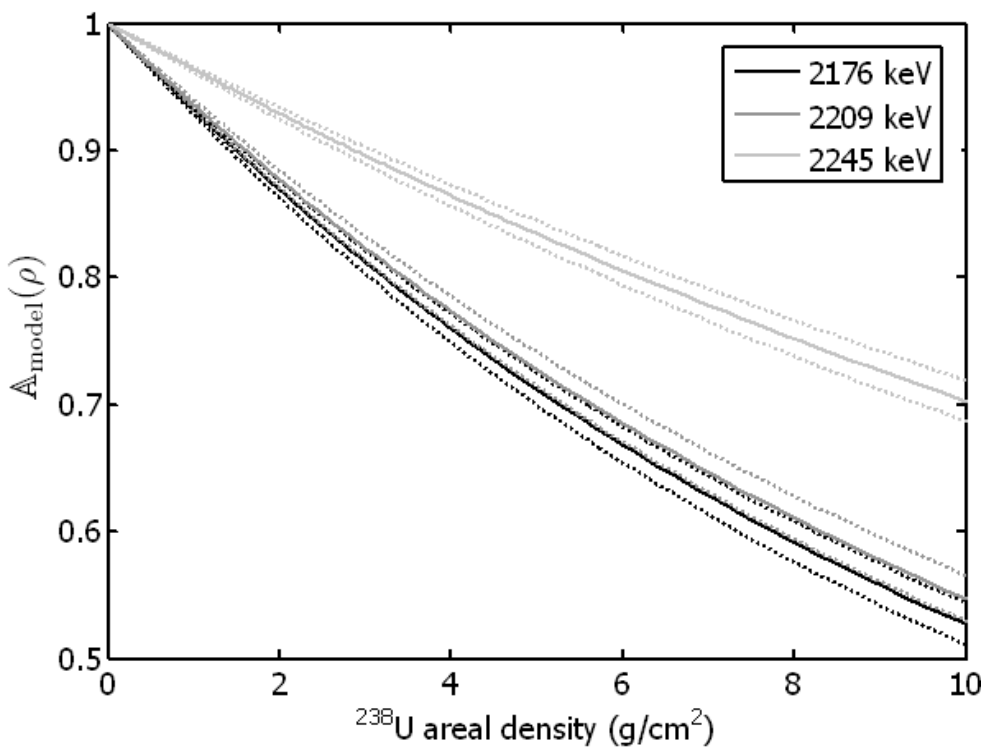

Figure A.5 Predicted values of $\mathbb{A}_{\text {model }}(\rho)$ for three strongest ${ }^{238} \mathrm{U}$ resonances measured. Dotted lines indicate $\pm 1 \sigma$ errors due to uncertainty in the strengths of the resonances from the Heil et. al. measurement. 
Table A.4 Values of the measured areal density of ${ }^{238} \mathrm{U}$ obtained by analysis of NRF peaks, and by direct measurement of the mass and area of the ${ }^{238} \mathrm{U}$ plates used in the assay target. The first errors listed for the NRF measurement are $1 \sigma$ errors due to counting statistics in this experiment. The second stated errors are due to the uncertainties in values of $\Gamma_{0}$ extracted from Heil et al.

\begin{tabular}{ccc}
\hline \hline Run & $\begin{array}{c}\text { NRF-measured } \rho x \\
\left(\mathrm{~g} / \mathrm{cm}^{2}\right)\end{array}$ & $\begin{array}{c}\text { direct } \rho x \\
\left(\mathrm{~g} / \mathrm{cm}^{2}\right)\end{array}$ \\
\hline 2 & $8.14 \pm{ }_{0.99}^{0.98} \pm 0.49$ & 8.47 \\
3 & $1.37 \pm 0.68 \pm 0.08$ & 1.69 \\
4 & $3.12 \pm{ }_{0.76}^{0.73} \pm 0.15$ & 3.34 \\
\hline
\end{tabular}

While previous transmission measurements using quasi-monoenergetic photon sources have indicated null results for the observation of notch refill[42], the data obtained in this experiment, using thick targets and a bremsstrahlung beam have exhibited a trend indicative of notch refill that increases measured NRF rates by up to $5 \%$ for large resonances and the target containing the most ${ }^{238} \mathrm{U}$. A correction based on the MCNPX modeling has been implemented in the analysis producing the areal densities measured by transmission NRF. Without the notch refill correction, the data tend to under-predict the areal density of ${ }^{238} \mathrm{U}$ in the target by approximately twice the standard deviation estimated by Poisson counting statistics.

The ${ }^{238} \mathrm{U}$ resonance parameters reported by Heil et al. tend to result in under-predicted ${ }^{238} \mathrm{U}$ areal densities, primarily due to the 2176 and $2209 \mathrm{keV}$ resonances, which were found to be slightly smaller than reported. This could also be due to a slightly larger notch refill correction than indicated by our modeling.

To within statistical uncertainties, the measurement agrees with models used to describe resonant attenuation and subsequent measurement of fluorescence emitted from the transmission detector. However, the results indicate the difficulty of using transmission nuclear resonance fluorescence to precisely measure small quantities of an isotope in an assay target. Rates at which statistics accrue in a transmission measurement are strongly dependent on the strengths of the resonances used for the measurement. Measurements of $\mathrm{NRF}$ in ${ }^{235} \mathrm{U}$ and ${ }^{239} \mathrm{Pu}$ have indicated their resonances are smaller than those examined here. This indicates that a transmission NRF measurement that is useful for nuclear safeguards applications would require a significantly more intense photon source than the $65 \mu \mathrm{A}$ bremsstrahlung beam used in this experiment. Higher current electron accelerators are commercially available[11]. However, required photon count rates pose difficult challenges for semiconductor detectors and energy resolution requirements make scintillating detectors less attractive. 


\section{B Used Fuel Radioactivity Calculations}

It is important to quantify the photon flux emanating from spent fuel assemblies and the associated background rates in the $\gamma$-ray detectors. Used fuel compositions and radioactive decay rates were determined using the computer code, OrigenARP 5.1.01 (referred to herein as Origen) that is distributed with the Scale 6 software package[39]. This code uses a series of cross section libraries that are calibrated to the neutronic environment of specific nuclear reactor designs to determine the production and depletion of isotopes in nuclear fuel during irradiation. Thereafter, Origen uses isotope half lives tabulated from the Table of Isotopes[40] to calculate radionuclide decay rates within the used fuel, assuming neutron-induced reactivity is negligible.

Because NRF is not highly sensitive to the concentration of trace isotopes that are not being investigated, it was decided that a suitable used fuel library would be a sampling of three different assembly types, all having undergone typical irradiations for used nuclear fuel. The compositions of used fuel that were considered for this work resulted from Origen calculations for the burn-up parameters provided in Table B.1.

The compositions of the irradiated and subsequently cooled fuel were calculated for several decay times up to 20 y. These compositions were used along with an Origen library to determine radioactive decay types and rates. Calculated rates of $\gamma$ ray emission due to nuclear de-excitation, photon creation due to $\beta$ ray induced bremsstrahlung, and $\gamma$ rays resulting from spontaneous fission were determined, histogrammed into $25 \mathrm{keV}$ wide energy bins, and used as a source spectrum for a subsequent MCNPX simulation. The MCNPX simulation considers the self-shielding of $\gamma$ rays in the spent fuel and any external shielding that is present in the NRF assay geometry, producing an expected photon spectrum that would be incident upon the region of a shielded $\gamma$-ray detector.

The total photon intensity $\sim 10$ years after discharge is a factor of $\sim 30$ smaller than that at discharge. The intensity above $1.6 \mathrm{MeV}$ decreases by a factor of $\sim 500$ over the same time. The peak at $1596 \mathrm{keV}$ seen in all specta is due to ${ }^{154} \mathrm{Eu}$, which has an $8.5 \mathrm{y}$ half-life. Higher-energy photons are primarily due to the decays of ${ }^{144} \mathrm{Pr}$ and ${ }^{106} \mathrm{Rh}$, both of which are short-lived daughters of isotopes that decay with half-lives of about $1 \mathrm{y}$. These isotopes may also emit energetic $\beta$ particles during radioactive decay. These $\beta$ particles account for a large fraction of the bremsstrahlung emitted from the spent fuel.

The production of many important background-inducing fission products will vary with reactor operating conditions and types. For example, assuming fission is induced by a thermal neutron

Table B.1 Burn-up parameters used for three assembly types in Origen calculation. IE indicates initial enrichment of uranium in the fuel, $M_{U}$ indicates mass of uranium in fuel, $B U$ indicates total irradiation of fuel or burn-up, and $P_{a v}$ indicates the average power at which the reactor was assumed to operate, which is proportional to neutron flux.

\begin{tabular}{ccccc}
\hline \hline $\begin{array}{c}\text { Assembly } \\
\text { Type }\end{array}$ & $\begin{array}{c}I E \\
(\%)\end{array}$ & $\begin{array}{c}M_{U} \\
(\mathrm{MT})\end{array}$ & $\begin{array}{c}\mathrm{BU} \\
(\mathrm{GWd} / \mathrm{MTU})\end{array}$ & $\begin{array}{c}P_{a v} \\
(\mathrm{MW} / \mathrm{MTU})\end{array}$ \\
\hline 17x17 PWR & 3.1 & 0.4249 & 45.00 & 27.397 \\
9x9 BWR & 1.91 & 0.1675 & 42.00 & 38.837 \\
VVER440 & 3.6 & 0.1198 & 38.29 & 32.894 \\
\hline
\end{tabular}




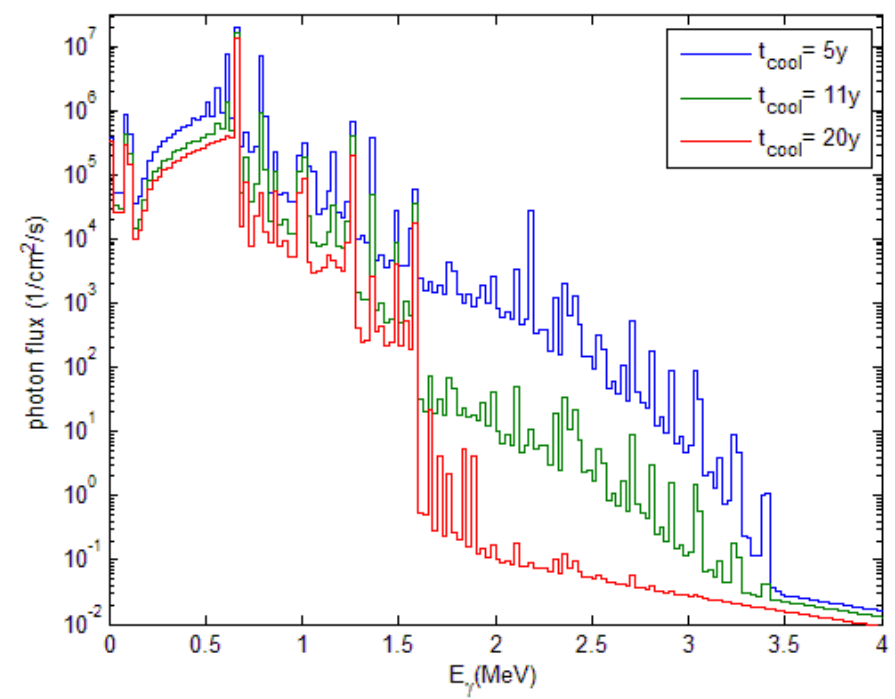

Figure B.1 Calculated average photon flux at $100 \mathrm{~cm}$ from fuel assembly center due to radioactive decay in $17 \mathrm{x} 17$ PWR fuel assembly and 45 GWd/MTU burn-up for cooling times of 5, 11, and $20 \mathrm{y}$. Energy bins are $25 \mathrm{keV}$ wide.

spectrum, the cumulative yield of ${ }^{106} \mathrm{Rh}$ from ${ }^{239} \mathrm{Pu}$ fission is approximately 10 times larger than from ${ }^{235} \mathrm{U}$ fission. Therefore longer irradiations or use of fuel that has high initial plutonium content will result in larger photon intensities between the energies of 1.6 and 3.6 MeV. Because of this variability, the fuel radioactivity values described in this section are for the purposes of providing a reference, rather than precise quantities.

\section{B.1 Unshielded Photon Flux}

Initially, the source term created by Origen was used to determine the photon flux at a hypothetical detector location due to the radioactive decay within a spent fuel assembly. The flux was determined by tallying all photons that crossed a cylindrical surface of $100 \mathrm{~cm}$ radius, centered on the considered fuel assembly. These flux spectra are shown in Figures B.1 - B.3 for the three assemblies indicated in Table B.1 for cooling times of 5, 11, and 20 y after discharge from the reactor.

The computed spectra for each fuel assembly type follow the same trend with time, and are also quite similar to one another. The PWR assembly produces the largest photon flux, but also contains the most fuel. Conversely, the PWR assembly produces the smallest flux per mass of fuel. The most intense $\gamma$-ray line is at $662 \mathrm{keV}$ due to ${ }^{137} \mathrm{Cs}$ decay. The peak at $1596 \mathrm{keV}$ due to ${ }^{154} \mathrm{Eu}$ is also of significance as this peak represents the most intense source of $\gamma$ rays in the energy range of interest for NRF. Above this energy, the primary contributors to the photon flux are ${ }^{144} \mathrm{Pr},{ }^{106} \mathrm{Rh}$ and spontaneous fission of ${ }^{244} \mathrm{Cm} .{ }^{144} \mathrm{Pr}$ and ${ }^{106} \mathrm{Rh}$ are short-lived $\beta^{-}$-emitters that are in secular equilibrium with their decay parents, both of which have approximately 1y half-lives. Discrete peaks that may be observed due to ${ }^{144} \mathrm{Pr}$ and ${ }^{106} \mathrm{Rh}$ decay are listed in Table B.2. The indicated half lives are those of the parent isotopes.

${ }^{244} \mathrm{Cm}$ has an 18 y half-life and contributes a continuum of photons that extends in energy up to $\sim 10 \mathrm{MeV}$. Empirical descriptions of spontaneous fission $\gamma$-ray spectra have been described 


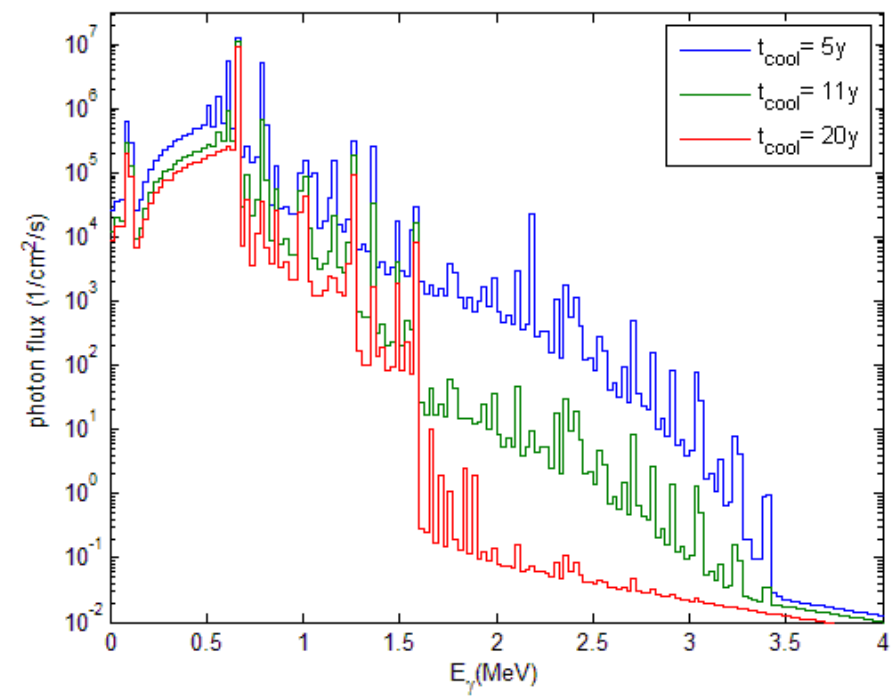

Figure B.2 Calculated average photon flux at $100 \mathrm{~cm}$ from fuel assembly center due to radioactive decay in 9x9 BWR fuel assembly and $42 \mathrm{GWd} / \mathrm{MTU}$ burn-up for cooling times of 5, 11, and $20 \mathrm{y}$. Energy bins are $25 \mathrm{keV}$ wide.

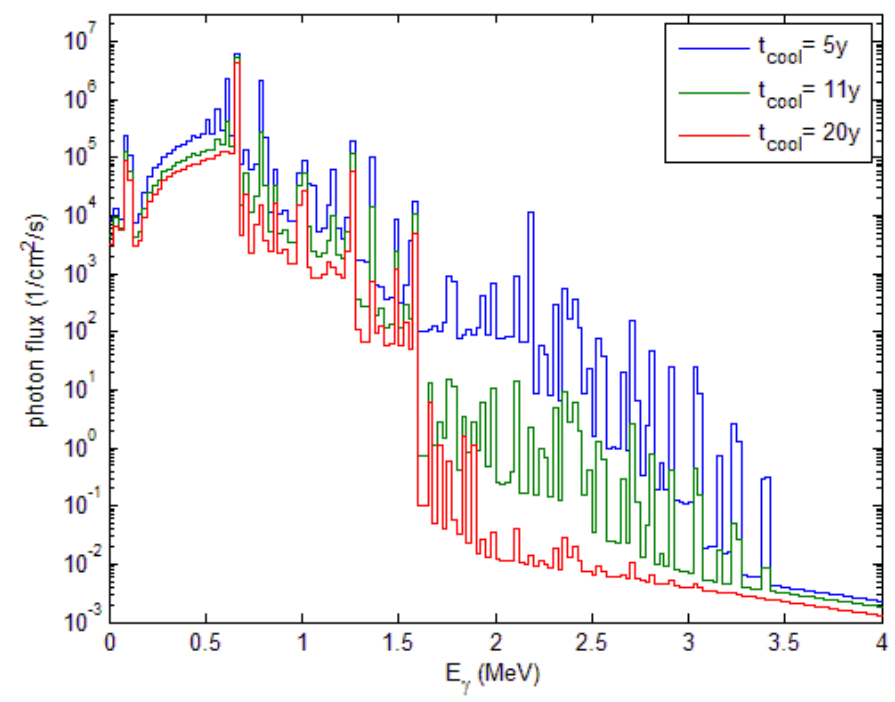

Figure B.3 Calculated average photon flux at $100 \mathrm{~cm}$ from fuel assembly center due to radioactive decay in VVER-440 fuel assembly and $38.3 \mathrm{GWd} / \mathrm{MTU}$ burn-up for cooling times of 5, 11, and $20 \mathrm{y}$. Energy bins are 25 $\mathrm{keV}$ wide. 
Table B.2 $\gamma$-ray energies and intensities of two important fission daughter isotopes in the Pu NRF energy region.

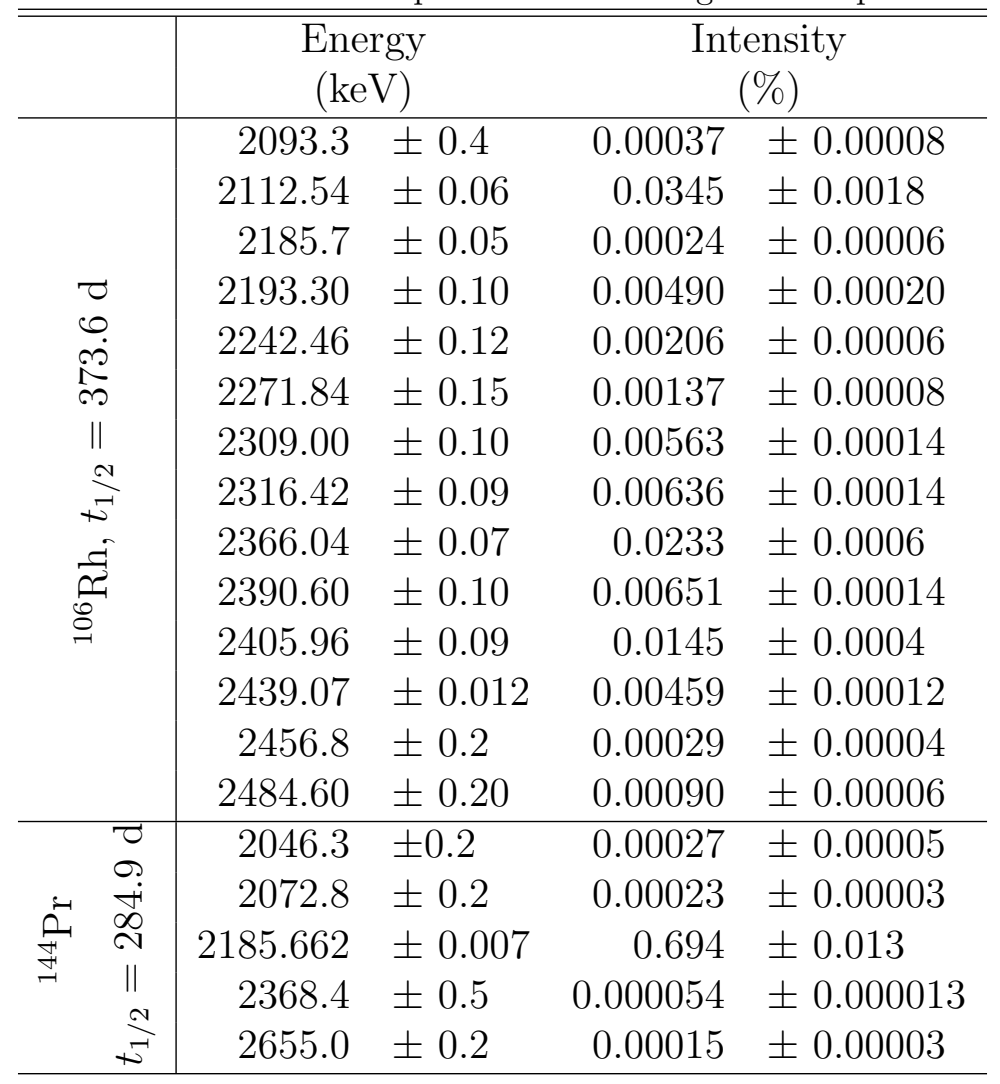


Table B.3 Calculated interaction rates for a 100\% relative efficiecy HPGe detector located $100 \mathrm{~cm}$ from the center of a $17 \times 17$ PWR assembly.

\begin{tabular}{ccc}
\hline \hline $\begin{array}{c}\text { Cooling } \\
\text { Time }(\mathrm{y})\end{array}$ & $\begin{array}{c}\text { Pb Shield } \\
\text { Thickness }(\mathrm{cm})\end{array}$ & $\begin{array}{c}\text { Interaction } \\
\text { Rate }(1 / \mathrm{s})\end{array}$ \\
\hline 5 & 0 & $2.7 \times 10^{9}$ \\
11 & 0 & $1.4 \times 10^{9}$ \\
20 & 0 & $9.8 \times 10^{8}$ \\
\hline 5 & 1.27 & $8.3 \times 10^{8}$ \\
11 & 1.27 & $4.2 \times 10^{8}$ \\
20 & 1.27 & $2.9 \times 10^{8}$ \\
\hline 5 & 2.54 & $2.6 \times 10^{8}$ \\
11 & 2.54 & $1.2 \times 10^{8}$ \\
20 & 2.54 & $8.3 \times 10^{7}$ \\
\hline 5 & 5 & $2.8 \times 10^{7}$ \\
11 & 5 & $1.2 \times 10^{7}$ \\
20 & 5 & $7.0 \times 10^{6}$ \\
\hline 5 & 7.5 & $4.1 \times 10^{6}$ \\
11 & 7.5 & $1.5 \times 10^{6}$ \\
20 & 7.5 & $7.5 \times 10^{5}$ \\
\hline 5 & 10 & $7.5 \times 10^{5}$ \\
11 & 10 & $2.6 \times 10^{5}$ \\
20 & 10 & $1.1 \times 10^{5}$ \\
\hline
\end{tabular}

in the Origen manual[39] and by Verbeke[41]. Above $2 \mathrm{MeV}$, the shapes of the two descriptions are almost identical, however the Origen description, which was used here is about $50 \%$ larger in overall magnitude than that of Verbeke. The Origen spontaneous fission $\gamma$-ray spectrum is given by the following:

$$
\begin{aligned}
N(E) & =11.5 \\
& =35.4 \exp [-1.78 E] \\
& =12.6 \exp [-1.09 E] \\
& =0
\end{aligned}
$$

$$
\begin{array}{r}
0.1<E \leq 0.6 \mathrm{MeV} \\
0.6 \leq E \leq 1.5 \mathrm{MeV} \\
1.5 \leq E \leq 10.5 \mathrm{MeV} \\
E \leq 0.1 ; E>10.5 \mathrm{MeV}
\end{array}
$$

The total photon interaction rates that an unshielded, $100 \%$ relative efficiency HPGe detector, located $100 \mathrm{~cm}$ from the center of a spent fuel assembly would experience due to the $\gamma$-ray flux shown in Figure B.1 is summarized in Table B.3. These rates are unacceptably high indicating that any spent fuel measurement involving traditional detectors will require significant shielding and collimation of the volume.

\section{B.1.1 Shielded Detector Configuration}

To determine the influence of shielding and collimation on expected count rates due to irradiated fuel assembly activity, a modified geometry was considered. In this geometry, a 17x17 fuel assembly was considered to be positioned into a corner of made up of $1 \mathrm{~m}$ thick $\mathrm{Pb}$ walls as shown in 

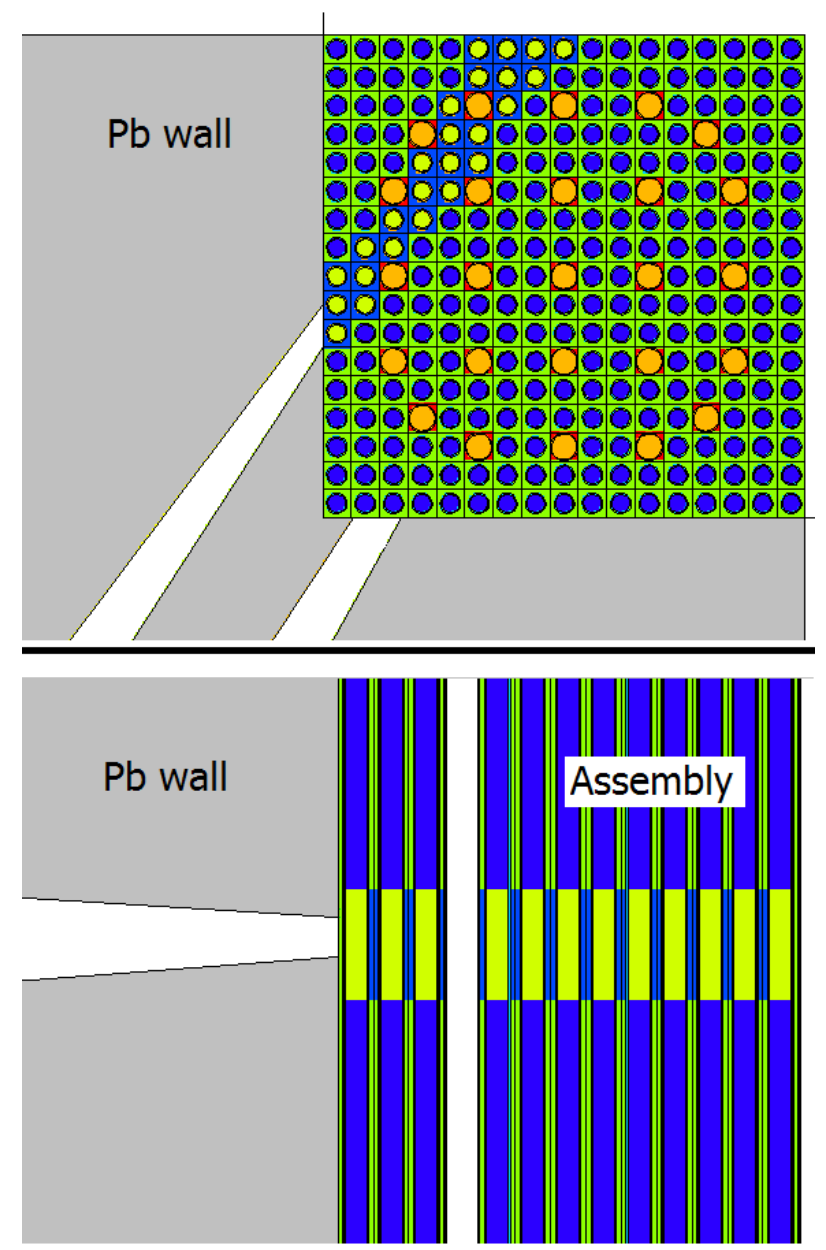

Figure B.4 Two views of heavily shielded radioactive background simulation geometry. The alternative shading indicates regions of the fuel assembly in which sampling of photon production was enhanced, with reduced weight.

Figure B.4. Two holes bored into the wall allow detectors to view a portion of the fuel assembly. The bores were modeled as truncated cones whose projected radii would decrease to $0.5 \mathrm{~cm}$ at the center of the fuel assembly. The maximum radius of the truncated right-angle cone was $4 \mathrm{~cm}$ at $1 \mathrm{~m}$ distance from the fuel pin. In our simulations photon production was biased such that more photons with reduced statistical weight were generated in pins in the differently shaded region to increase the likelihood that simulation of photons contributed to the calculated photon spectrum.

The spectrum resulting from this shielded configuration is reduced in intensity by approximately a factor of 1000 because only a fraction of the total fuel assembly that is within the field of view of the collimated detector. Simulated spectra for a 100\% relative efficiency HPGe detector located behind 5, 7.5 and $10 \mathrm{~cm}$ of $\mathrm{Pb}$ at a distance of $100 \mathrm{~cm}$ from the center of the fuel assembly are shown in Figure B.5. The expected overall count rates are $1.7 \times 10^{5}, 2.2 \times 10^{4}$, and $3.8 \times 10^{3}$ counts/s, respectively. Even with this collimated viewing arrangement, approximately 3" of lead would need to be located directly between a HPGe detector and the fuel assembly to limit the detector count rate due to radioactive emissions to $10^{4} \mathrm{cps}$.

Likewise, with this shielding configuration (2" - 3" $\mathrm{Pb}$ and collimation to $1 \mathrm{pin})$, we anticipate a count rate of $10^{-3}-10^{-2}$ counts $/ \mathrm{keV} / \mathrm{s}$ in the ${ }^{239} \mathrm{Pu} \mathrm{NRF}$ energy range $(2-2.5 \mathrm{MeV})$ due to 


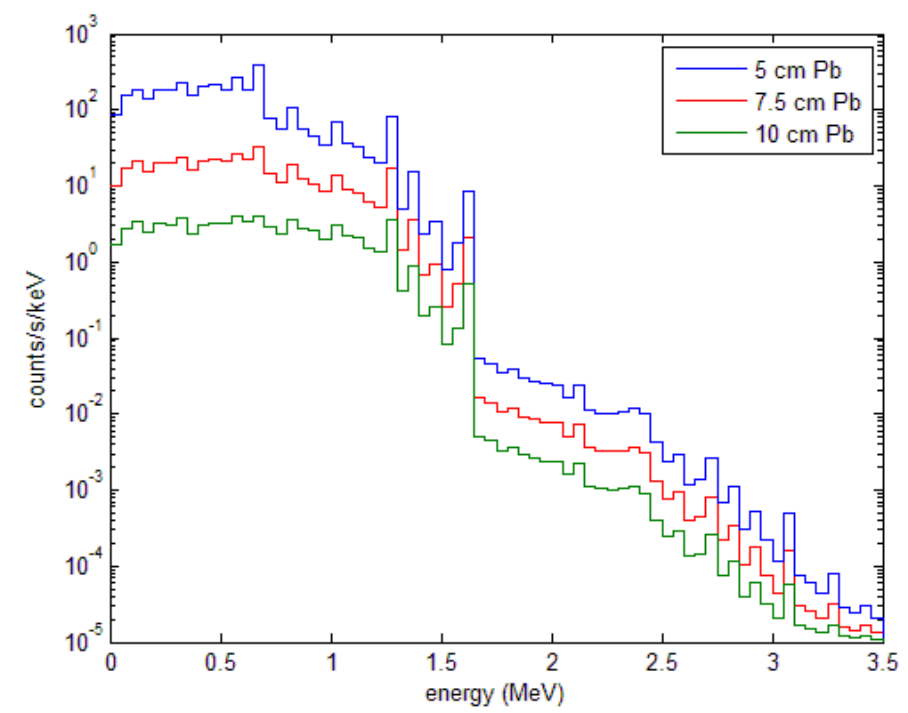

Figure B.5 Calculated spectra due to spent fuel radioactivity expected to be measured by a collimated $100 \%$ relative efficiency HPGe detector located $100 \mathrm{~cm}$ from the center of a spent fuel assembly and behind $5,7.5$ and $10 \mathrm{~cm}$ of $\mathrm{Pb}$. Note activity due to $(\mathrm{n}, \gamma)$ reactions has been neglected.

radioactive decay of spent fuel. A significant fraction of the detector counts will be due to photon interactions that only deposit part of the photon energy and therefore build up a continuous background. This continuum is compared to the NRF rates in Section 4.1.

\section{B.1.2 Simplifying Assumptions}

The photon flux that would be produced due to $(n, \gamma)$ reactions has not been directly considered due to its dependence upon shielding materials used near the assembly. It is anticipated, however, that use of neutron thermalizers and absorbers near the assembly would render the neutron capture $\gamma$ ray insignificant. A single $17 \times 17$ PWR assembly emits from $4 \times 10^{8}$ to $1 \times 10^{8}$ neutrons/s as it cools from $t_{\text {cool }}=5-20 \mathrm{y}$. Considering that this rate is $10^{-7}$ that of $\gamma$-ray emission, we can comfortably neglect this contribution. Furthermore, capture lines for neutrons incident upon all natural isotopes of $\mathrm{Pb}, \mathrm{W}, \mathrm{Ge}, \mathrm{La}$, and $\mathrm{Br}$ were explicitly examined and no lines at energies of known NRF were present in the database[56].

The calculated spectra shown in Figures B.1 - B.3 assumed the fuel assembly as constituent materials homogenized into a block with the assembly's outer dimensions. To check the impact of this simplification a time-intensive simulation was conducted where the fuel assembly consisted of numerous discrete pins. The difference in the calculated emitted photon spectrum is negligible except at very low energies. A comparison of the spectra (without the $\beta$-induced bremsstrahlung) for two identical compositions, one homogenized and the other including each discrete pin, is shown as Figure B.6. The spectra are essentially identical above $500 \mathrm{keV}$, below this energy the spectrum produced in the detailed geometry is more intense than the homogenized geometry by a factor of up to 3 , increasing to approximately 30 with decreasing energy. This is due to the fact that fuel is contained within zircalloy cladding which attenuates low-energy $\gamma$ rays, whereas the homogenized spent fuel allows source particles to leave the assembly after penetrating arbitrarily small thicknesses of material. This would be significant were it not for the fact that NRF mea- 


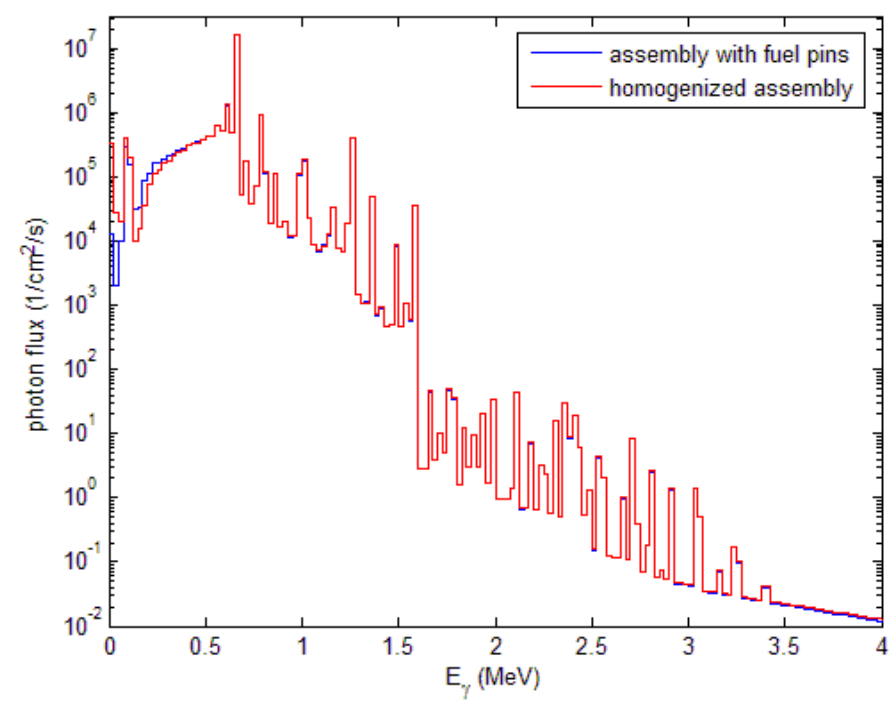

Figure B.6 Calculated average photon flux at $100 \mathrm{~cm}$ from fuel assembly center due to radioactive decay in $17 \mathrm{x} 17$ PWR fuel assembly and $45 \mathrm{GWd} / \mathrm{MTU}$ burn-up for $11 \mathrm{yr}$ cooling time. Both spectra have $25 \mathrm{keV}$ wide bins. The blue spectrum assumes that the radioactive source term is uniformly distributed throughout the $\mathrm{UO}_{2}$ matrix of fuel pins, whereas the red spectrum assumes that the fuel assembly is homogenized and the the source term is distributed throughout the entire assembly.

surement geometries include a filter to significantly reduce the intensity of low-energy photons, which reduces the sensitivity of the measurements to inaccuracies in the predicted low-energy background spectrum.

Because of this, we can generally assume with reasonable precision that the spent fuel activity that a detector will be sensitive to is given by the total assembly activity times the fractional volume of spent fuel is within a detector's line of sight through the $1 \mathrm{~m}$ shielded wall. 


\section{Finite Element Treatment of Target Geometry}

The effective geometric attenuation can be easily computed for more complicated geometries using a finite element method. Returning to equation 3.2, and assuming that photon down-scatter can be neglected, we observe that $\Phi(E, \mathbf{r})$ is given by a combination of beam divergence and exponential attenuation of the incident flux over the length a photon must travel before interaction at the finite element point, $r_{i}$. Therefore, we find

$$
\Phi(E, \mathbf{r}) \approx \Phi_{i}(E) \exp \left[-\mu(E) r_{i}\right]\left[\frac{d_{s}}{d_{s}+r_{i}}\right]^{2}
$$

where $d_{s}$ is the distance between the photon source and the front of the target. The quantity $\left[d_{s} /\left(d_{s}+r_{i}\right)\right]^{2}$ represents the divergence of the interrogating beam. The dimension $r_{o}$ and $r_{d}$ are easily determined for each finite element point. For an element grid point $(i, j, k)$, values of $r_{i}, r_{o}$, $\theta$, and $r_{d}$ are calculated and substituted into the geometric attenuation expression to obtain an energy-differentiated NRF $\gamma$-ray count rate:

$$
\begin{aligned}
\frac{d R}{d E}=\sum_{i} \sum_{j} \sum_{k} N \sigma_{\mathrm{NRF}}(E) \Phi_{i}(E) P_{f}(E) A \epsilon(E) \exp \left[-\mu(E) r_{i}^{i, j, k}\right] \exp \left[-\mu_{n r} r_{o}^{i, j, k}\right] \\
\left(\frac{d_{s}}{d_{s}+r_{i}^{i, j, k}}\right)^{2} W\left(\theta^{i, j, k}\right) \frac{f^{i, j, k}}{4 \pi\left(r_{d}^{i, j, k}\right)^{2}} \Delta^{i} \Delta^{j} \Delta^{k}
\end{aligned}
$$

where $\Delta^{i}, \Delta^{j}$ and $\Delta^{k}$ refer to spatial dimensions of each finite element and $f^{i, j, k}$ indicates the volume fraction of the element that is contained within the target object. When a constant cross section approximation is assumed, and $P_{f}(E)$ and $\epsilon(E)$ are evaluated at the resonance energy, we obtain

$$
\begin{gathered}
R=\sum_{i} \sum_{j} \sum_{k} N \sigma_{\mathrm{NRF}}^{C} \Gamma_{D} \Phi_{i} P_{f} A \epsilon \exp \left[-N \sigma_{\mathrm{NRF}}^{C} r_{i}^{i, j, k}\right] \exp \left[-\mu_{n r}\left(r_{i}^{i, j, k}+r_{o}^{i, j, k}\right)\right] \\
\left(\frac{d_{s}}{d_{s}+r_{i}^{i, j, k}}\right)^{2} W\left(\theta^{i, j, k}\right) \frac{f^{i, j, k}}{4 \pi\left(r_{d}^{i, j, k}\right)^{2}} \Delta^{i} \Delta^{j} \Delta^{k} \\
\equiv N \sigma_{\mathrm{NRF}}^{C} \Gamma_{D} \Phi_{i} A \epsilon \times G
\end{gathered}
$$

where $G$ includes the sum of the products of all terms that contain a parameter that varies between finite elements.

An example of the geometric correction, $G$ as a function of spatial coordinate is shown in Figure C.1. In this example, we assume that a $1 \mathrm{~cm}$ radius sphere of $\mathrm{UO}_{2}$ is irradiated by a uniform beam of photons incident along the x-axis. The ${ }^{235} \mathrm{U}$ abundance of the uranium is assumed to be 0.05 and the energy of the photon beam is assumed to be that of the centroid of the $1.733 \mathrm{MeV}$ resonance in ${ }^{235} \mathrm{U}$, which has an effective natural width, $g \Gamma_{0}=34 \mathrm{meV}$. The detector is positioned 


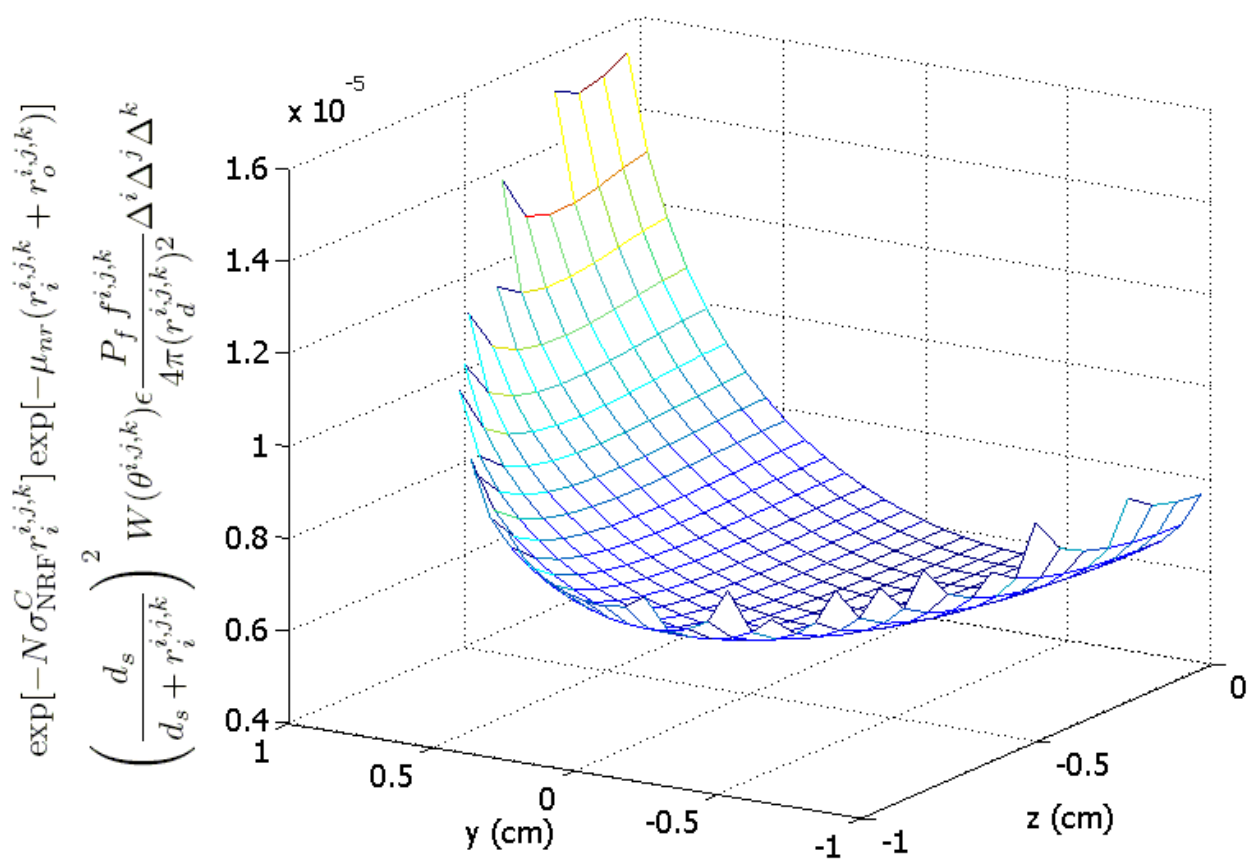

Figure C.1 Grid-point dependent quantities, $G^{i, j, k}$ of equation C.3 for $i=15$. See text for details.

in the $\mathrm{x}-\mathrm{y}$ plane, $30 \mathrm{~cm}$ from the center of the sphere and at an angle of $\theta=135^{\circ}$, relative to the interrogating beam direction. The calculation was performed for one half of the z-dimension coordinates because of symmetry. The sphere was divided into a $30 \times 30 \times 15$ grid, and the values plotted are the grid-point-dependent quantities of equation C.3 for the plane $i=15$, assuming $P_{f}=1$, and $W(\theta)=1$. The $f^{i, j, k}$ were assumed to be unity if the center of the corresponding finite element was within the sphere, and zero otherwise. The summed geometric factor is $G=0.0579$ $\mathrm{cm}$, yielding an expression for the expected $\mathrm{NRF}$ count rate of $0.0579 \mathrm{~cm} \times N \sigma_{\mathrm{NRF}}^{C} \Gamma_{D} \Phi_{i} A \epsilon$. This $\mathrm{NRF} \gamma$-ray detection rate is the same as would be expected from irradiation of $1 \mathrm{~cm}^{2}$ of a 0.0579 $\mathrm{cm}$ thick pure ${ }^{235} \mathrm{U}$ slab, neglecting photon attenuation in the target. It is also 0.276 times the rate that would be estimated if attenuation due to non-resonant interactions in the target sphere were ignored. 


\section{Rayleigh Scattering in MCNP}

\section{D.1 Introduction}

Documentation of the MCNP series of codes has claimed for years to accurately represent the data present in evaluated nuclear data files [5, 29, 47, 48]. The photo-atomic data in the ENDF/BVI format are based on the evaluated photon data library (EPDL97)[49]. However, the work supporting this report observed that the sections of code describing the form factor evaluation of coherent and incoherent scattering had not been updated since their precursor code, MCP, was written in 1973[50]. This legacy code results in significant inaccuracies in photon spectra computed by MCNPX when simulating geometries that are typical of NRF experiments[51].

MCNP treats all coherent scattering as Rayleigh scattering of unpolarized photons and follows the form factor approximation for the Rayleigh cross section:

$$
\sigma_{R a y}(\theta)=\sigma_{T h}(\theta)[F(q, Z)]^{2}
$$

where $F(q, Z)$ is a tabulated form factor value, and $\sigma_{T h}(\theta)$ is the Thomson scatter cross section, given by $\sigma_{T h}(\theta)=\frac{r_{e}^{2}}{2}\left(1+\cos ^{2} \theta\right)$. Here, $r_{e}^{2}$ is the classical electron radius, $2.818 \mathrm{fm}$, and $\theta$ is the angle between the scattered and incident photon.

Data libraries used in MCNP are created by the code NJOY, which reads ENDF tabulations of atomic form factors and converts them into the ACE format used in MCNP[30]. Atomic form factor values are tabulated for a given $Z$ and momentum transfer between the scattered photon and the atomic electron, $q$. The units of $q$ should be units of momentum, however $q$ has traditionally been tabulated in reduced wave number, which has units of inverse length ${ }^{1}$. The derivation and use of momentum transfer values in MCNP has been poorly documented. MCP and MCNP user manuals describes $q$ in units of $\mathrm{cm}^{-1} ;[48,50]$ however, the data tabulated in MCNPX are in units of $\AA^{-1}[52]$.

The legacy problems in elastic scatter come from the fact that MCNP has historically had a fixed array of $q$-values for which coherent scattering form factors, $C(Z, q)$ are defined. Despite the fact that the EPDL97 (and ENDF) now tabulates form factors for $q$ up to $10^{11} \AA^{-1}, q$ in MCNP was restricted to $q \leq 6 \AA^{-1}$ and coherent scattering form factors for $q>6$ were assumed to be 0. Table D.1 demonstrates the maximum coherent scattering angles, $\theta_{\max }$, that were allowed by this restriction. Figure D.1 helps to explain why this short-coming had gone un-noticed by many MCNP users ${ }^{2}$. In it, the fraction of the Rayleigh scattering cross section that is due to scattering

\footnotetext{
${ }^{1}$ The conversion between momentum transfer, $\hbar k \sin (\theta / 2)$, and reduced wave number is accomplished by dividing by Plank's constant, $h$, inserting $\frac{c}{c} \frac{m_{e} c^{2}}{m_{e} c^{2}}$, equating, $E=\hbar c k$ and, $\sin (\theta / 2)=\sqrt{\frac{1-\cos \theta}{2}}$. Evaluating the constants:

$$
q=\frac{c}{h c} \frac{m_{e} c^{2}}{m_{e} c^{2}} 2 \hbar k \sin (\theta / 2)=\frac{E}{m_{e} c^{2}} \frac{2 m_{e} c^{2}}{\sqrt{2} h c} \sqrt{1-\cos \theta}=29.1445\left[\AA^{-1}\right] \frac{E}{m_{e} c^{2}} \sqrt{1-\cos \theta}
$$

${ }^{2}$ This short-coming was independently observed by Lodwick and Spitz when they compared in vivo $\mathrm{x}$ ray fluorescence measurements of lead in bone to MCNP calculations[53].
} 
Table D.1 Maximum coherent scattering angles allowed in the MCNP codes.

\begin{tabular}{rr}
\hline \hline$E(\mathrm{keV})$ & Angle \\
\hline 74.4 & $180^{\circ}$ \\
105 & $90^{\circ}$ \\
194 & $45^{\circ}$ \\
853 & $10^{\circ}$ \\
1733 & $4.9^{\circ}$ \\
2423 & $3.5^{\circ}$ \\
\hline
\end{tabular}

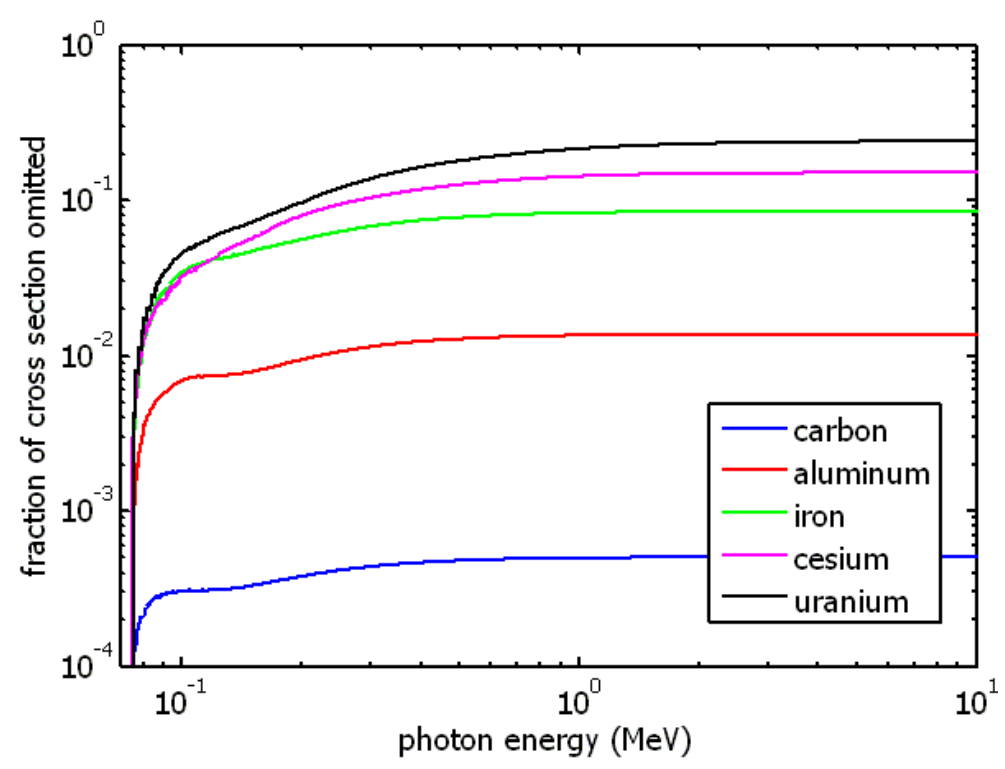

Figure D.1 Fraction of Rayleigh scattering cross section that corresponds to a reduced momentum transfer value, $q \geq 6 \AA^{-1}$.

above $\theta_{\max }$ is plotted versus photon energy for various elements, i.e.,

$$
f=\frac{\int_{q=6\left[\AA^{-1}\right]}^{q_{\max }} \sigma_{R a y}(\theta) d \boldsymbol{\Omega}}{\int_{q=0}^{q_{\max }} \sigma_{\text {Ray }}(\theta) d \boldsymbol{\Omega}}
$$

where $q_{\max }=29.1445\left[\AA^{-1}\right] \frac{E}{m_{e} c^{2}} \sqrt{2}$. The omission is most significant in high- $Z$ materials, and is most important when photon energies exceed approximately $100 \mathrm{keV}$. As will be described, this omission is only important when coherently-scattered photons can be distinguished from incoherently-scattered and annihilation photons. The distinction is most obvious when backscattering of multi-MeV photons from high- $Z$ materials is considered, and this is precisely what is done in experiments designed to measure NRF.

Modification of the MCNPX source code and the NJOY data processing code are described in Section D.4. These modifications enable MCNPX to simulate Rayleigh scattering for all problems.

To test that modifications of the MCNPX code and datafiles were successful, two pairs of simulations were conducted comparing elastic scattering rates before and after implementation of the modifications. For each pair a pencil beam of monoenergetic $1.7 \mathrm{MeV}$ photons was normally impinged upon a $1 \mathrm{~cm}$ radius by $100 \mathrm{~mm}$ depth cylindrical target of either uranium or iron. 
Photons emitted from the target were tallied as they crossed a spherical surface of $10 \mathrm{~cm}$ radius, centered at the center of the target cylinder. The direction of the emitted photons relative to the direction of the incident beam were tallied in $1^{\circ}$ angular bins up to $10^{\circ}$, and then in $5^{\circ}$ bins. The energy of the emitted photons were also tallied, with photons within $860 \mathrm{eV}$ of the initial energy being considered elastically scattered. This bin width was selected to ensure that incoherently scattered photons are not tallied into the elastic bin for scattering angles greater than $1^{\circ}$. The effective elastic scattering cross section was then calculated by the relation

$$
\frac{d \sigma_{\mathrm{ES}}}{d \Omega} \approx \frac{N_{\mathrm{F} 1}}{\Delta \Omega} \frac{M}{N_{A} \rho x}
$$

where $N_{\mathrm{F} 1}$ is the number of photons in the elastic tally bin, $\Delta \Omega$ is the solid angle subtended by the angular bin, $M$ is the molar mass of the target material, $N_{A}$ is Avogadro's number, and $\rho x$ is the areal density of the target.

Figures D.2 and D.3 present the simulated angle-differentiated cross sections for elastic scattering of $1.7 \mathrm{MeV}$ photons before (red) and after (black) the implementation of the extended Rayleigh scattering cross section library. The EPDL97 cross section for elastic scattering is also shown in green in both figures. The cross sections resulting from simulations that include the new form factor arrays closely follow the EPDL97 values. For the red histogram, non-zero values calculated for scattering into angles above the critical angle of $5.2^{\circ}$ are due to the relatively improbable combination of photoelectric absorption followed by bremsstrahlung emission. This process yields photons in the elastic bin, and would yield an even smaller (nominally) elastic scattering probability were the highest energy bin made narrower. Regardless, the five (uranium) and seven (iron) decade decreases in effective elastic scattering cross section between the $3^{\circ}-4^{\circ}$ and $6^{\circ}-7^{\circ}$ bins indicate effects of the non-physical cutoff that had been implemented in MCNP. That the effective cross section was calculated to be $30 \%$ (uranium) and $8.5 \%$ (iron) lower after implementation of the extended library for the range $1^{\circ}-5^{\circ}$ is explained by the fact that photons probabilistically sampled to undergo coherent scattering had been constrained to the forward $5.2^{\circ}$, whereas now all angles are physically allowed. The amount by which MCNPX had overpredicted the Rayleigh scattering cross section is exactly the inverse of the fractions shown in Figure D.1 for $1.7 \mathrm{MeV}$ photons. Thus, as another result of the truncated form factor arrays, MCNP had previously been

overestimating elastic scattering of photons in forwards directions, and this effect was most severe for high photon energies and high- $Z$ materials.

\section{D.2 Form Factor Sampling Methodology}

This section describes the method used in MCNPX to sample the coherent scatter cross section. This method defines the integrated form factors, which must also be included in the ACE files that provide the photo-atomic data for MCNP.

Suppose we want to sample a probability density function $(\mathrm{PDF}), P(y)$, defined in the interval $a \leq y \leq b$, and related to another known PDF, $Q(y)$ by the following

$$
P(y)=C_{0} F(y) Q(y)
$$

where $C_{0}$ is a constant, $C_{0}>1$ and $F(y)$ is a function with range $0 \leq F(y) \leq 1$. 


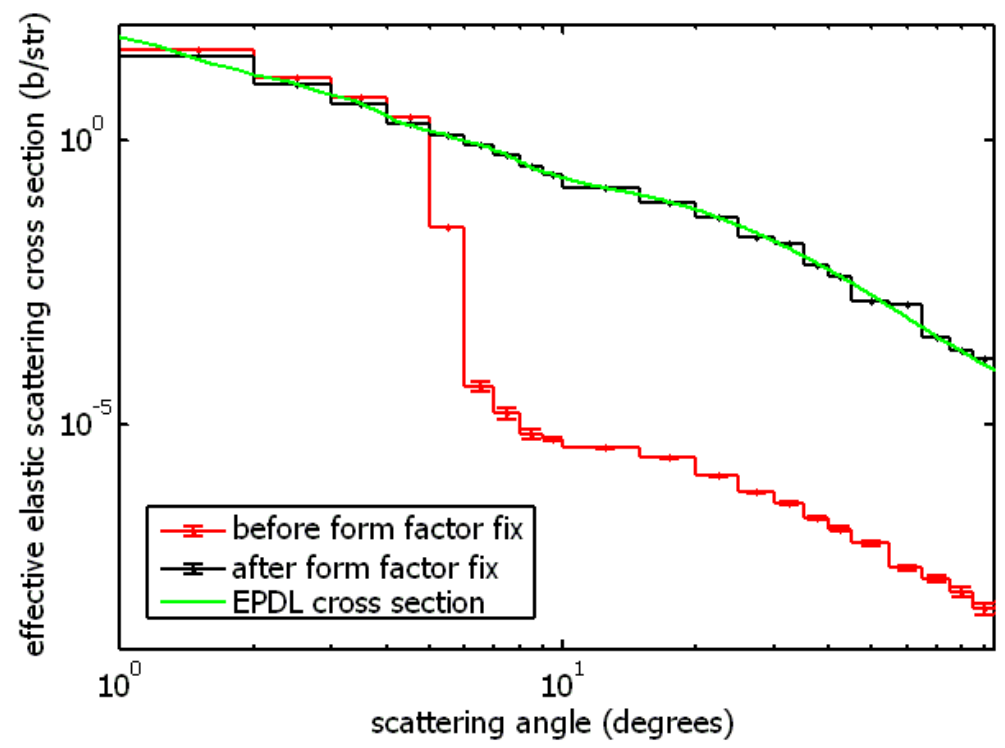

Figure D.2 MCNPX-simulated differential cross section for scattering of $1.7 \mathrm{MeV}$ photons within $860 \mathrm{eV}$ of initial energy from $100 \mu \mathrm{m}$ thick uranium with EPDL Rayleigh scattering cross section overlaid.

\section{D.2.1 Sampling a Simple PDF}

First we must define what is meant by 'sampling a PDF'. Assume the PDF, $Q(y)$, is a normalized and integrable function in some interval,

$$
\int_{a}^{b} Q(y)=1
$$

Sampling of $Q(y)$ is accomplished by using a random number, $0 \leq r \leq 1$, to select a value, $y_{\text {sample }}$, between $a$ and $b$ with a probability proportional to the value of the PDF.

The cumulative (or integrated) distribution function (CDF) for $Q(y)$ is defined as,

$$
\mathbf{Q}\left(y^{\prime}\right)=\int_{a}^{y^{\prime}} Q(y) d y
$$

Therefore $\mathbf{Q}(a)=0$ and $\mathbf{Q}(b)=1$. By inverting the function $\mathbf{Q}\left(y^{\prime}\right)$, we have a function with a domain of $[0,1]$. Using the random number, $r_{1}$, we obtain

$$
y_{\text {sample }}=\mathbf{Q}^{-1}\left(r_{1}\right)
$$

which is a value of $y$, sampled from the PDF $Q(y)$. Clearly, this only works for PDFs that can be integrated, and whose integral can be inverted.

\section{D.2.2 Rejection-Sampling Method}

Sampling of equation D.4 is accomplished by use of the rejection-sampling method. First, $Q(y)$ is sampled. The value of $y$ is accepted, with a probability of $F(y)$, by using a second random number, $r_{2}$ to check that

$$
r_{2}<F(y)
$$




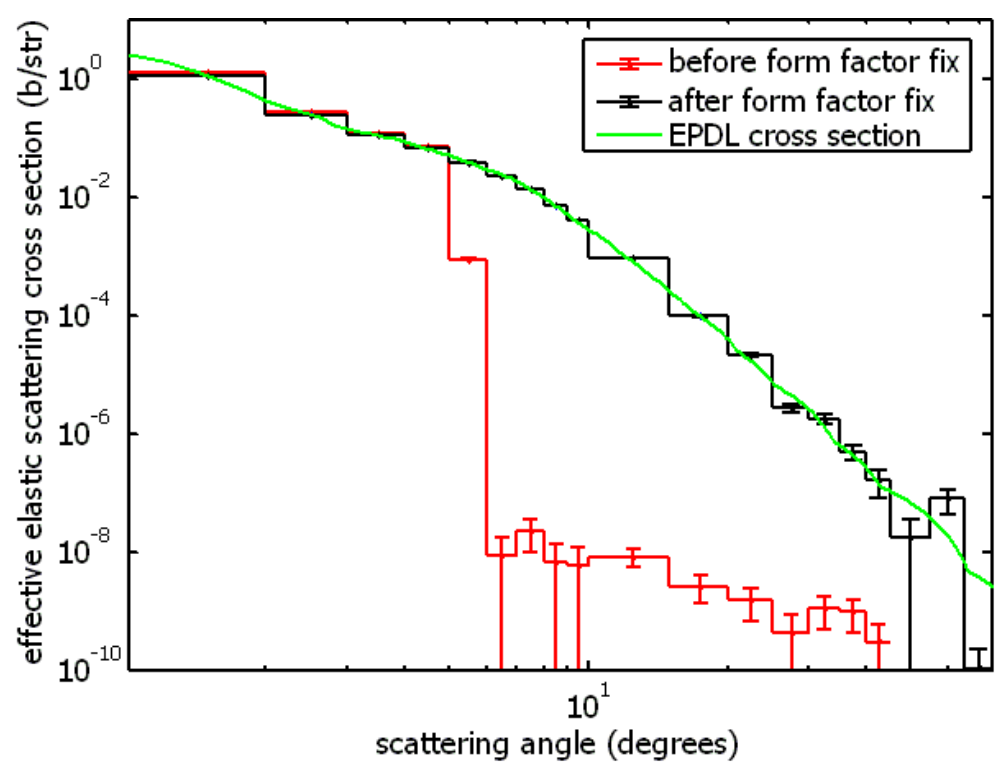

Figure D.3 MCNPX-simulated differential cross section for scattering of $1.7 \mathrm{MeV}$ photons within $860 \mathrm{eV}$ of the initial energy off of $100 \mu \mathrm{m}$ thick iron with EPDL Rayleigh scattering cross section overlaid The large statistical uncertainty at high angles is due to the rarity of the events.

The probability of not accepting a sampled value for $y$ is given by

$$
P_{\text {reject }}=1-\int_{a}^{b} F(y) Q(y) d y
$$

When $y$ is rejected, we sample another value of $Q(y)$ and again check with a new random number against equation D.8, potentially allowing an infinite number of samplings.

To prove that this will give an appropriate sampling of $P(y)$, first note that

$$
1=\int_{a}^{b} P(y) d y=\int_{a}^{b} C_{0} F(y) Q(y) d y
$$

so

$$
\int_{a}^{b} F(y) Q(y) d y=C_{0}^{-1}
$$

The probability of $k$ rejections is then

$$
P_{k}=\left(1-C_{0}^{-1}\right)^{k}
$$

and the probability of accepting the $k+1$-th iteration is

$$
\left(1-1 / C_{0}\right)^{k} F(y)
$$

Using

$$
\sum_{k=0}^{\infty}\left(1-1 / C_{0}\right)^{k}=C_{0}
$$

we see that if an infinite number of iterations is allowed, the PDF for sampling $y$ is given by

$$
C_{0} F(y) Q(y)=P(y)
$$




\section{D.3 Sampling Rayleigh Scattering Cross Sections}

The angle of Rayleigh scattering is sampled in MCNPX by the rejection-sampling method. In this formulation, the independent variable, $y$, is given by $q^{2}$, the square of the momentum transfer of the scattering event, which for a given photon energy is

$$
q=K \alpha \sqrt{1-\mu}
$$

where $K=29.1445 \AA^{-1}, \alpha=E / m_{e} c^{2}$, and $\mu=\cos (\theta)$.

The Rayleigh scattering cross section is given by

$$
\sigma_{\text {Ray }}(\theta)=\sigma_{T h}(\theta)[F(q, Z)]^{2}
$$

where the Thomson scattering cross section is given by

$$
\sigma_{T h}(\theta)=\frac{r_{e}^{2}}{2}\left(1+\cos ^{2} \theta\right)=\frac{r_{e}^{2}}{2}\left(1+\mu^{2}\right)
$$

The PDF for scattering into the angle, $\mu$, is given by

$$
p(\mu)=\frac{\sigma_{\text {Ray }}(Z, \alpha, \mu)}{\int \sigma_{\text {Ray }}(Z, \alpha, \mu) d \mu}
$$

The PDF for a given squared-momentum transfer is given by

$$
P\left(q^{2}\right) d\left(q^{2}\right)=p(\mu)\left|\frac{d \mu}{d\left(q^{2}\right)}\right| d\left(q^{2}\right)
$$

Solving equation D.16 for $\mu$ and differentiating, we have

$$
\frac{d \mu}{d\left(q^{2}\right)}=\frac{-1}{K \alpha^{2}}
$$

Substituting, equations D.17, D.18, D.19, and D.21 into equation D.20, we obtain the expression for the angular scattering PDF

$$
P\left(q^{2}\right)=\frac{r_{e}^{2}}{(K \alpha)^{2}} \frac{1+\mu^{2}}{2} \frac{[F(q, Z)]^{2}}{\int \sigma_{\text {Ray }}(Z, \alpha, \mu) d \mu}
$$

In MCNPX, equation D.22 is re-expressed by multiplying numerator and denominator by pairs of $Z^{2}$ and $\int_{0}^{q_{\max }^{2}}\left[F\left(Z, q^{2}\right)\right]^{2} d\left(q^{2}\right)$ to obtain

$$
P\left(q^{2}\right)=\left[\frac{r_{e}^{2} Z^{2} \int_{0}^{q_{\max }^{2}} Z^{-2}\left[F\left(Z, q^{2}\right)\right]^{2} d\left(q^{2}\right)}{(K \alpha)^{2} \int \sigma_{\text {Ray }}(Z, \alpha, \mu) d \mu}\right]\left[\frac{1+\mu^{2}}{2}\right]\left[\frac{[F(q, Z)]^{2} Z^{-2}}{\int_{0}^{q_{\max }^{2}}\left[F\left(Z, q^{2}\right)\right]^{2} Z^{-2} d\left(q^{2}\right)}\right]
$$

This cumbersome expression is actually written in the form of equation D.4, where the expression in the first brackets

$$
C_{0}=\frac{r_{e}^{2} Z^{2} \int_{0}^{q_{\max }^{2}} Z^{-2}\left[F\left(Z, q^{2}\right)\right]^{2} d\left(q^{2}\right)}{(K \alpha)^{2} \int \sigma_{\text {Ray }}(Z, \alpha, \mu) d \mu}
$$


is, in fact, a constant. The integral, $\int \sigma_{\text {Ray }}(Z, \mu) d \mu$, is the total Rayleigh scattering cross section and the other integral is obtained through interpolation between tabulated integrated form factor values. The expression in the second brackets of equation D.23 is

$$
F(y)=\frac{1+\mu^{2}}{2}
$$

which is a function with range, $[0,1]$. The third brackets contain,

$$
Q(y)=\frac{[F(q, Z)]^{2} Z^{-2}}{\int_{0}^{q_{\max }^{2}}\left[F\left(Z, q^{2}\right)\right]^{2} Z^{-2} d\left(q^{2}\right)}
$$

which is the PDF for selecting a squared momentum transfer, where again, the tabulated integrated form factor is used.

\section{D.3.1 Integrated Form Factors}

The quantity,

$$
\int_{0}^{q_{\max }^{2}} Z^{-2}\left[F\left(Z, q^{2}\right)\right]^{2} d\left(q^{2}\right)
$$

is the integrated form factor. It is numerically integrated in NJOY and values are tabulated for corresponding values of $q^{2}$. The ENDF form factor tabulation specifies that $\ln \left[F\left(Z, q^{2}\right)\right]$ shall be linearly interpolated with $\ln \left[q^{2}\right]$. Thus the integrated form is tabulated as

$$
\int_{0}^{q}{ }^{2} Z^{-2}\left[F\left(Z, q^{2}\right)\right]^{2} d\left(q^{2}\right) \approx \exp \left(\sum_{i} \ln \left[Z^{-2}\left[F_{i}\left(Z, q_{i}^{2}\right)\right]^{2}\right] \Delta\left[\ln \left(q_{i}^{2}\right)\right]\right)
$$

The interpolation scheme is slightly more complicated in that $\ln (0)$ is undefined, and the first momentum transfer entry, $q_{1}=0$. Such problems are fixed in NJOY by the use of linear interpolation as necessary. Likewise, the ENDF creators were aware of this, and correspondingly recommend linear interpolation in such cases.

\section{D.4 Description of MCNPX Patch 'Rayleigh Fix'}

The modification described here successfully allowed MCNPX to simulate Rayleigh scattering properly, but it has been superseded by the modifications described in reference[45], which use the same logic, but also dynamically allocate variables such that the data used in MCNPX is identical to that in the EPDL97 libraries.

A description of the sampling method used to determine the scatter angle of a photon that underwent Rayleigh scattering is described in Section D.2. MCNP uses tabulated values of $C\left(Z, q^{2}\right)$ for each element, $Z$, and for fixed array of a momentum transfer values, $q$. The length of these tabulations was defined by the variable, $\mathrm{MCOH}=55$ words. Two arrays whose contents are defined during MCNPX initialization, VCO and WCO contained the corresponding values of $q$ and $q^{2}$, respectively. The maximum value in VCO was $q=6 \AA^{-1}$. The EPDL97 contains values of $F(Z, q)$ for $q \leq 10^{11} \AA^{-1}$. To enable MCNPX to simulate Rayleigh scattering at all possible angles for energies of interest, new arrays of length MCOHE $=77$ words were also defined in 
MCNPX initialization. They contained the original 55 values of $q$ and $q^{2}$, as well as 22 additional values, increasing quickly to $q=10^{4} \stackrel{\circ}{A}^{-1}$. These arrays were named VCOE and WCOE. The only additional changes necessary to the MCNPX code was a logical check of the length of the form factor arrays (part of the JXs array). If the new form factor array had a length of MCOHE, the code would perform the exact same logic for sampling the coherent scatter PDF as is done in the un-modified code, except that the form factor array and integrated form factor arrays would be defined according to $q$ and $q^{2}$ values of VCOE and WCOE, respectively.

The PERL scripting of the MCNPX patch follows:

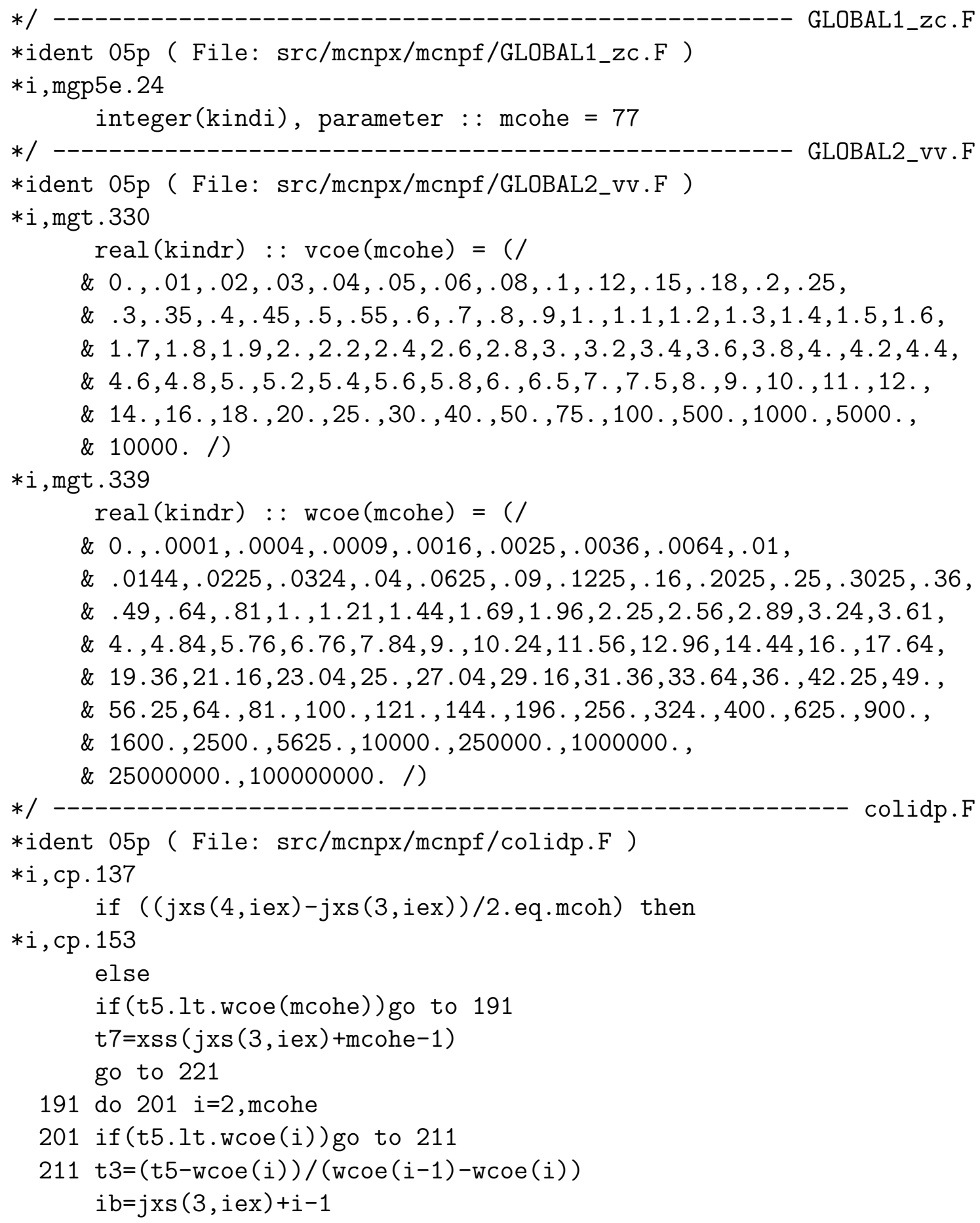




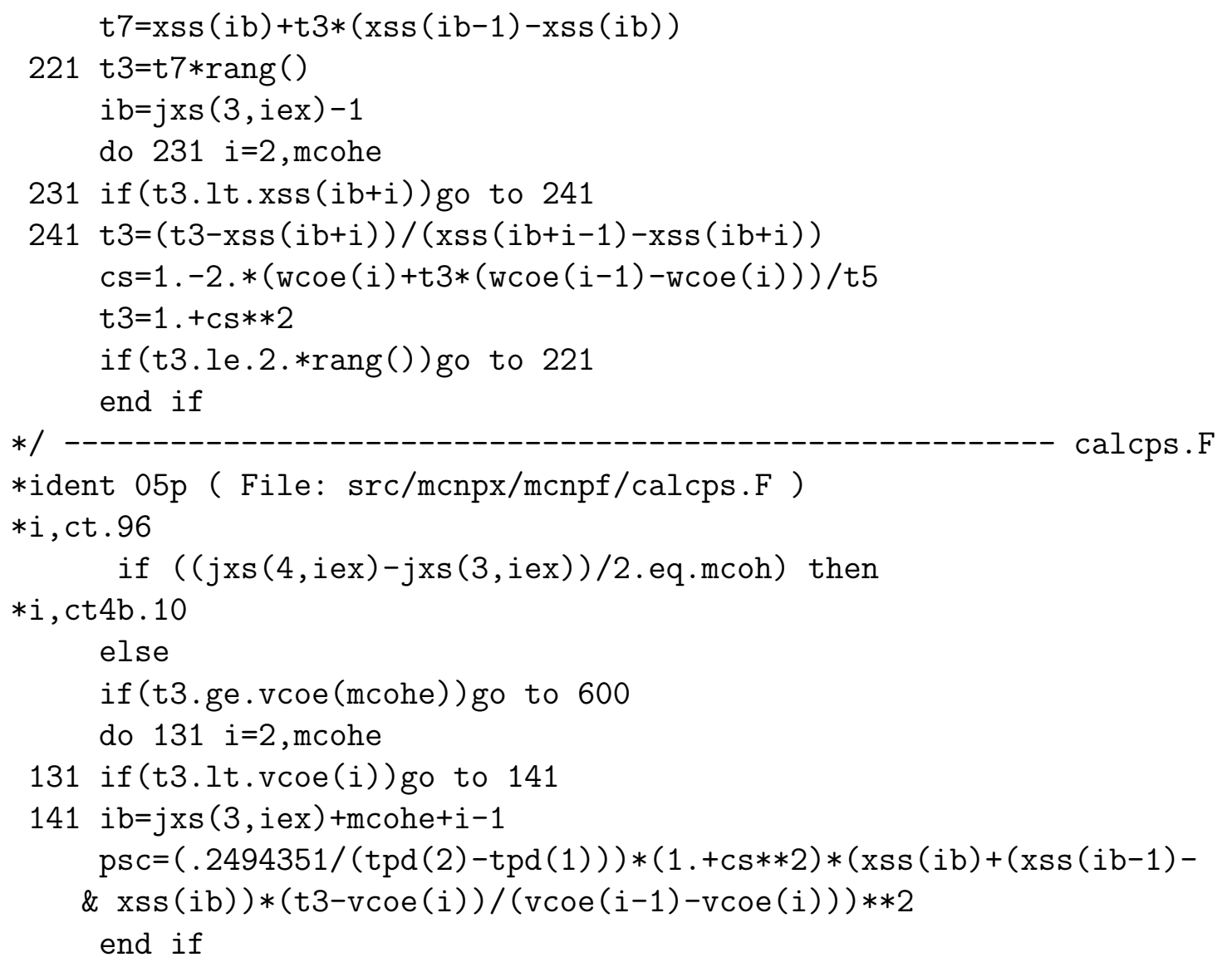

The data processing code, NJOY, must also be modified in order to create data files that contain form factor and integrated form factor arrays that reflect the MCNPX changes. This process was quite simple. The array VCO in MCNPX corresponds to an array vC in the ACER routine of NJOY. This array is modified, and subsequent assumptions of the length of $\mathrm{VC}$ by the following NJOY patch, created to modify NJOY259:

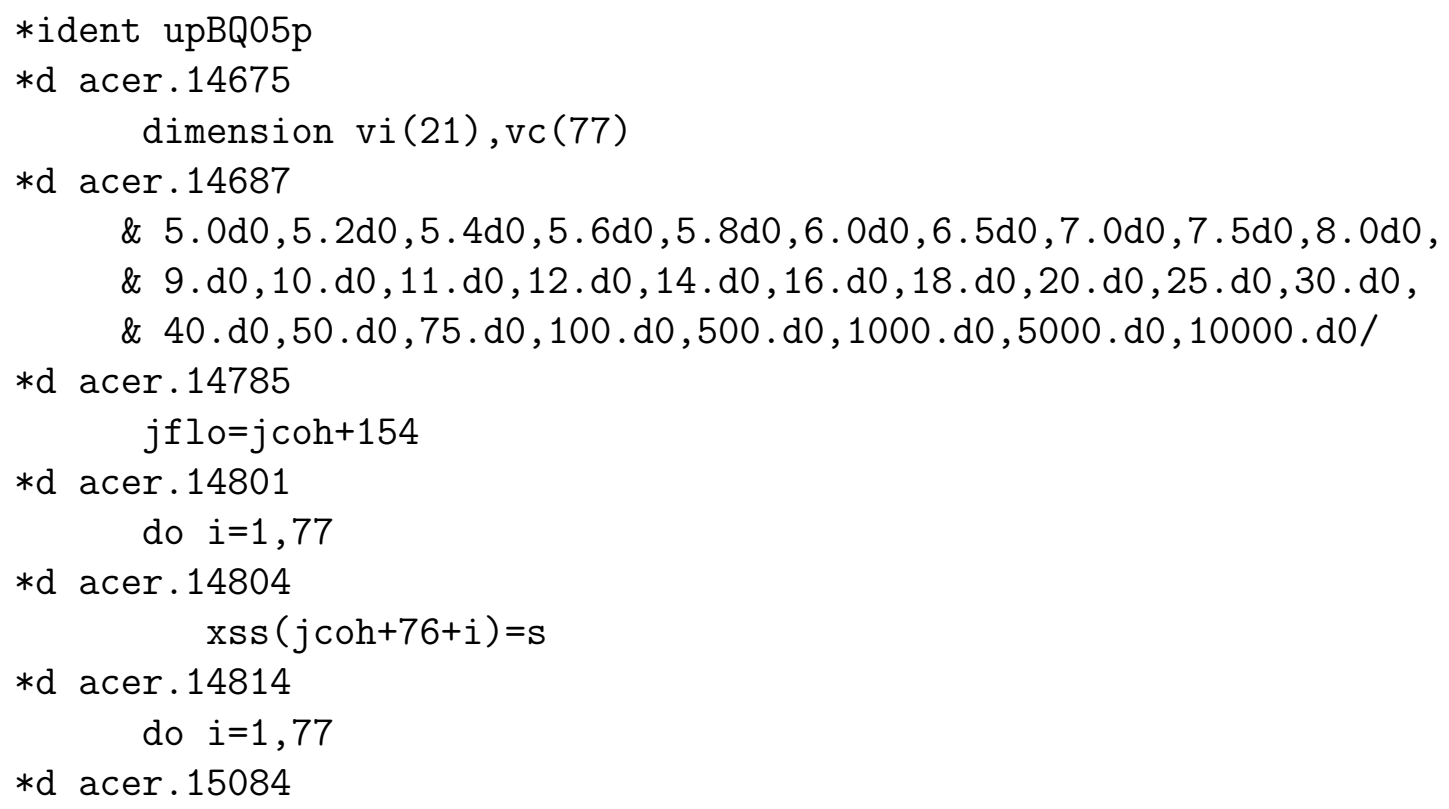


write (nsyso,' $\left.(1 \mathrm{x}, 1 \mathrm{p}, 6 \mathrm{e} 14.4)^{\prime}\right)(\operatorname{xss}(j \operatorname{coh}-1+i), i=1,77)$

$* \mathrm{~d}$ acer. 15088

write(nsyso, ' $\left.(1 \mathrm{x}, 1 \mathrm{p}, 6 \mathrm{e} 14.4)^{\prime}\right)(\mathrm{xss}(\mathrm{jcoh}+76+i), i=1,77)$

$* \mathrm{~d}$ acer. 15142

$\mathrm{n}=21+2 * 77$

Unfortunately, NJOY259 appears to have a bug that causes the program to crash in a latter section in which formatted x-ray fluorescence data tables are produced. These tables are supposed to follow the form factor array portion of the ACE file. To bypass this problem, each new ACE file, containing additional form factor data, that was produced by the modified NJOY259 code was combined with properly formatted $\mathrm{x}$ ray data tables in the previously distributed ACE files. This resulted in properly functioning data files that appropriately represent the Rayleigh scattering evaluated data present in the EPDL97. 


\section{E Addition of NRF Data to Photonuclear Datafiles}

This appendix describes the inclusion of NRF data into the photonuclear ENDF and ACE datafiles used by MCNPX. Because of constraints regarding both the ENDF data format and the NJOY code, several approximations were made, modifying the theoretical shape of the NRF resonances and eliminating correlated $\gamma$-ray emission cascades. After describing the process by which ENDF and ACE datafiles were created, the ramifications of these approximations are discussed.

\section{E.1 Description of Datafile Creation Method}

The capability of simulating photonuclear interactions has been in place within MCNPX since version 2.3[54]. These interactions include photo-fission, other photonuclear interactions such as $(\gamma, \mathrm{n})$ reactions, and in principle, $\left(\gamma, \gamma^{\prime}\right)$ reactions. In the most recent release of MCNPX (version 2.6.0)[5], descriptions of $\left(\gamma, \gamma^{\prime}\right)$ interactions were absent from the photonuclear data files. Below, is summarized the work that has been performed to add NRF data - which is a subset of the $\left(\gamma, \gamma^{\prime}\right)$ reactions - to photon transport in MCNPX. The data retrieval, analysis, and insertion is accomplished by the computer code NRFXSSI.EXE (NRF $\operatorname{cross}(\mathrm{X})$ SectionS Inserter), which was written for this project.

The process of adding NRF data to the ENDF-B/VII[29] and the MCNPX data file format, which are called ACE files, consists of the following steps:

1. NRF Data literature search. The Discrete Level Scheme Library (DLSL) of the Reference Input Parameter Library 2 (RIPL-2)[55] was used as the primary database of NRF levels. For each isotope, the corresponding Evaluated Nuclear Structure Data File (ENSDF) and documents referred to therein were also reviewed to search for more NRF lines.

2. A computer code, RIPL2FIX.EXE was written and used to improve the resolution with which energies of emitted $\gamma$ rays are described in the RIPL-2 DLSL. The program also modifies the DLSL database to take into account the fraction of the decay energy that is taken as recoil kinetic energy by the nucleus during $\gamma$-ray emission.

3. NRFXSSI step 1: information gathering

4. NRFXSSI step 2: NRF level identification and analysis.

5. NRFXSSI step 3: DLSL Level Trimming

6. NRFXSSI step 4: $\gamma$-ray cascade analysis

7. NRFXSSI step 5: data formatting

8. NRFXSSI step 6: data insertion into photonuclear ENDF file

9. ACE file creation using NJOY

10. NJOY bug repair. 
More detailed descriptions of the above process follow. Step 1 is done by hand. Step 2 is accomplished by invoking the program RIPL2FIX.EXE, which was written for this project. Steps 3-8 are accomplished by NRFXSSI.EXE. Step 9 involves operating the nuclear data processing code NJOY[30], and step 10 is post-processing of the NJOY output because the code was found to have a minor bug when creating photonuclear ACE files from ENDF files where photon creation processes are present more than once within the data file.

\section{E.1.1 NRF Data Search}

For each isotope used in this report, the $\operatorname{ENSDF}[56]$ and documents describing $\left(\gamma, \gamma^{\prime}\right)$ reaction studies cited therein were examined ${ }^{1}$. If levels not contained in the DLSL were identified and were within energy ranges deemed important for NRF-based assay, the state, its half-life, and its decay scheme were added to a modified DLSL file. Scientific literature post-dating the ENSDF cutoff date are also reviewed by searching the INSPEC[57] database with the key words 'nuclear resonance fluorescence' and the isotope being updated. The values of $E_{0}$, and $g \Gamma_{0}$ are required to calculate the total NRF cross section of a resonance. The product, $g \Gamma_{0}$ is often provided in the ENSDF, but the spin of the NRF state and therefore $g$ cannot be individually measured. In this case the spin is assumed to be a single unit greater than the ground state spin, and the value of $\Gamma_{0}$ that preserves the measured $g \Gamma_{0}$ is entered into the modified DLSL. ${ }^{2}$

\section{E.1.2 NRF $\gamma$-ray Energy Description and ripl2fix.exe}

The DLSL describes the excitation energies of nuclear states to a precision of $1 \mathrm{eV}$, although these values are often not known to this precision. The energies of $\gamma$ rays corresponding to the de-excitation of an excited nuclear level are only described to a precision of $1 \mathrm{keV}$. This could result in emission of NRF $\gamma$ rays at the resonance energy, which would dramatically alter the transport of NRF $\gamma$ rays. To correct for this, a program named RIPL2FIX.EXE was written.

RIPL2FIX.EXE modifies DLSL files by calculating de-excitation $\gamma$-ray centroid energies to 10 meV precision. After calculating these energies, RIPL2FIX re-writes modified DLSL files with higher $\gamma$-ray energy precision. RIPL2FIX.EXE accomplishes this through the following steps:

RIPL2FIX.EXE reads through each DLSL file located in the /LEVELS/ directory. It first reads the nominal level energies and mass number for each isotope. Then the energy of the $\gamma$ ray emitted during the de-excitation of the parent state to a daughter state (which may or may not be the ground state) is computed by the following equation:

$$
E_{\gamma}=E_{\text {parent }}-E_{\text {daughter }}-\frac{\left(E_{\text {parent }}-E_{\text {daughter }}\right)^{2}}{2 M c^{2}}
$$

where $M c^{2}$ is the rest mass-energy of the $\gamma$-emitting nucleus. The energy lost to the recoiling nucleus is $9 \mathrm{eV}$ for a $2 \mathrm{MeV}$ transition from a nucleus with mass number $A=238$. This mass is approximated as the product of $A$ and the mass of a neutron. This effectively neglects nuclear binding energies, however these differences would result in less than a $0.5 \%$ correction. Likewise,

\footnotetext{
${ }^{1}$ Many more ENDF and ACE files have since been created, but data searching has not been conducted for these isotopes.

${ }^{2}$ Since NFRXSSI.EXE currently assumes isotropic emission of NRF $\gamma$ rays, the spins of NRF states are presently un-important. If the code is improved to consider angular dependence of emitted $\gamma$ rays, unknown spin would result in potentially incorrect emitted $\gamma$-ray angular distributions.
} 
Table E.1 CASCADE and Dataset array formats. Descriptions of the variables are described in the text.

\begin{tabular}{lccccc}
\hline \hline & \multicolumn{5}{|c}{ Column \# } \\
Row type & {$[0]$} & {$[1]$} & {$[2]$} & {$[3]$} & {$[4]$} \\
\hline 1st Row & $\mathrm{N}_{\text {Levels }}$ & IB & $\theta$ & $A$ & 0 \\
Level Row & $N \gamma D$ & $E_{\text {Level }}$ & LN & $\tau$ & $J$ \\
Subsequent $N \gamma D$ Rows & -1 & $E_{\gamma}$ & daughter LN & $P_{\gamma}$ & 0 \\
\hline
\end{tabular}

our expression for the energy of the $\gamma$ ray is not exact: it is obtained from a second order binomial expansion of the quadratic equation that is solved when conservation of momentum and energy are imposed upon the two particle problem of photon emission from an isotope. The next term in the binomial expansion is $\frac{\left(E_{\text {parent }}-E_{\text {daughter }}\right)^{3}}{4 M^{2} c^{4}}$, which for the previous example is $40 \mu \mathrm{eV}$. The output modified DLSL files are created in the directory /OUT/. Compilation has been performed using CYGWIN $\mathrm{C}++[58]^{3}$.

\section{E.1.3 NRFXSSI Step 1: Information Gathering}

The NRFXSSI program is run by the routine NRFXSSI.CPP. This routine only calls subroutines that perform the steps described. In the first subroutine called, GATHER.CPP, the user is asked to identify the name of the isotope to be analyzed. After the specified isotope is located in the DLSL, the width, spin, de-excitation modes, and corresponding daughter levels are loaded into memory for each excited level. The Debye temperature of the elemental material and the ground state spin of the isotope are also loaded into memory. GATHER.CPP returns thee arrays to the main program: ATOMINF is a 3 element array containing the elemental symbol, Z, and A of the isotope; the other two arrays are DATASET and CASCADE. The layout of these arrays is depicted in Table E.1 and the content is described below.

The first row of CASCADE and DATASET is a header that contains information specific to the isotope being processed. $\mathrm{N}_{\text {Levels }}$ is an integer describing the number of nuclear levels described in the DLSL for the isotope. IB is a Boolean, which is 1 if the isotope has an even number of both protons and neutrons, and zero otherwise (at this point, this information is un-used by NRFXSSI). $\theta$ is the Debye temperature of the material in Kelvin, and $A$ is the isotope's mass number. After the header row, a Level Row follows. Each Level Row describes a specific nuclear level defined in the DLSL. $N \gamma D$ is the number of different $\gamma$-ray emissions this level may undergo. LN is an integer describing the level, $\tau$ is the mean lifetime of the level, in seconds, and $J$ is the spin of the level. After each Level Row, $N \gamma D$ rows follow, describing each $\gamma$ ray emitted from the level. The first entry in the $\gamma$ ray row is always a -1 . The energy of the emitted $\gamma$ ray is given as $E_{\gamma}$. The daughter's level is LN, and the probability for emission of the $\gamma$ ray is given by $P_{\gamma}$. After the $N \gamma D \gamma$-ray emission rows, another Level Row follows, until all $\mathrm{N}_{\text {Levels }}$ have been listed.

At this point in the code, DATASET and CASCADE only differ by the ordering of the Level Rows. CASCADE is ordered from highest to lower energy, whereas in DATASET, they are ordered as read from the DLSL. This facilitates subsequent program flow.

\footnotetext{
${ }^{3}$ The version of $\mathrm{C}++$ used to compile this was produced by Cygnus Solutions Inc. Cygwin is now owned by Redhat, and the version used to produce the programs described herein appears to be no longer distributed.
} 


\section{E.1.4 NRFXSSI Step 2: DLSL Data Trimming}

After the data are collected into the arrays, DATASET and CASCADE, both arrays are trimmed of unnecessary levels. Each level in DATASET is subjected to six checks:

1. If the daughter of the highest-energy $\gamma$ ray emitted from a level is not the ground state, the level is removed from the data array.

2. If no $\gamma$-ray data is known, the level is removed from the data array.

3. If the lifetime of the level is unknown, it is removed from the data array. The lifetime of the state must be known because it is related to the NRF cross section, which must be known.

4. If the spin of the level is unknown, it is removed from the data array. Often, the quantity, $g \Gamma_{0}$, is experimentally measured, instead of the spin and lifetime. It is important to note that values of $J$ and $\tau$ must be assigned or the code will purge the state.

5. If the spin of the level is greater than 10 it is removed from the data array. This removal triggers a printed warning in the code execution window.

6. If the probability that a state emits a $\gamma$ ray resulting in direct de-excitation to the ground state state is less than an adjustable tolerance level (set to 0.001 in the code), the level is removed from the data array. This removal triggers a printed warning in the code execution window.

For each level that is removed from DATASET, the subsequent $N \gamma D$ rows corresponding to $\gamma$-ray emission information for the level are also removed.

CASCADE also undergoes a series of checks to purge unnecessary data, however, since a state may be relevant for an NRF cascade, yet not directly excited by NRF absorption, different checks are necessary:

1. Identical to \#2 for DATASET.

2. If a level is higher energy than the highest-energy level in the trimmed DATASET array, the entire level is removed from the data array.

3. Lines corresponding to individual $\gamma$ rays are also purged from cascade if the intensity associated with the $\gamma$-ray emission is less than the tolerance of 0.001 .

\section{E.1.5 NRFXSSI Step 3: NRF Cross Section Analysis}

The cross section for NRF to occur for each resonance is calculated in the subroutine NRFCALC.CPP. This routine determines a cross section function for each excited state in the trimmed DATASET array. First the subroutine asks the user to define a temperature at which the cross section will be calculated. The code assumes that the cross section is either calculated from a Maxwell-Boltzmann distribution, equation 2.12, whose width is defined by the Doppler width of the resonance, equation 2.14, or the natural width of the NRF line, $\Gamma$, whichever is wider. The actual profile is given by equation 2.16, and the code should be modified to reflect this, but for the uses of the code to date, values of $\Gamma$ describing the NRF lines have been trivially small, and 
therefore can be accurately neglected. The ambient temperature, $T_{a}$, at which Doppler-broadened cross sections have been calculated is $300 \mathrm{~K}$ in all the produced ENDF files. The temperature used in equation 2.14 has been modified by

$$
T=T_{a} \cdot\left[0.906 \exp \left(0.1555 /\left(T_{a} / \theta\right)\right)\right]
$$

to take into account the effects of crystal lattices. Equation E.2 is only valid for $T_{a} \gg \theta$, and the code should be modified to instead include a numerical integration of equation 2.17.

Because of constraints in both NJOY and the ENDF format, data points can be defined on an energy grid no finer than $2 \mathrm{eV}$. If the width of the cross section distribution is greater than $6 \mathrm{eV}$, an energy grid spacing of $\Gamma / 2$ is used. This coarse energy resolution causes Gaussian distributions, whose full width at half maximum (FWHM) is normally $1-2 \mathrm{eV}$, to be highly prone to discretization errors that will alter the total integrated cross section during the discretization process. To correct this, the resonant integral is analytically computed in the code, and the pointwise integrated cross section values are multiplied by the ratio of the analytic integral to that of the discretized function. This ensures that the integrated cross section is correct. A schematic description of the discretization and renormalization process is shown in Figure E.1.
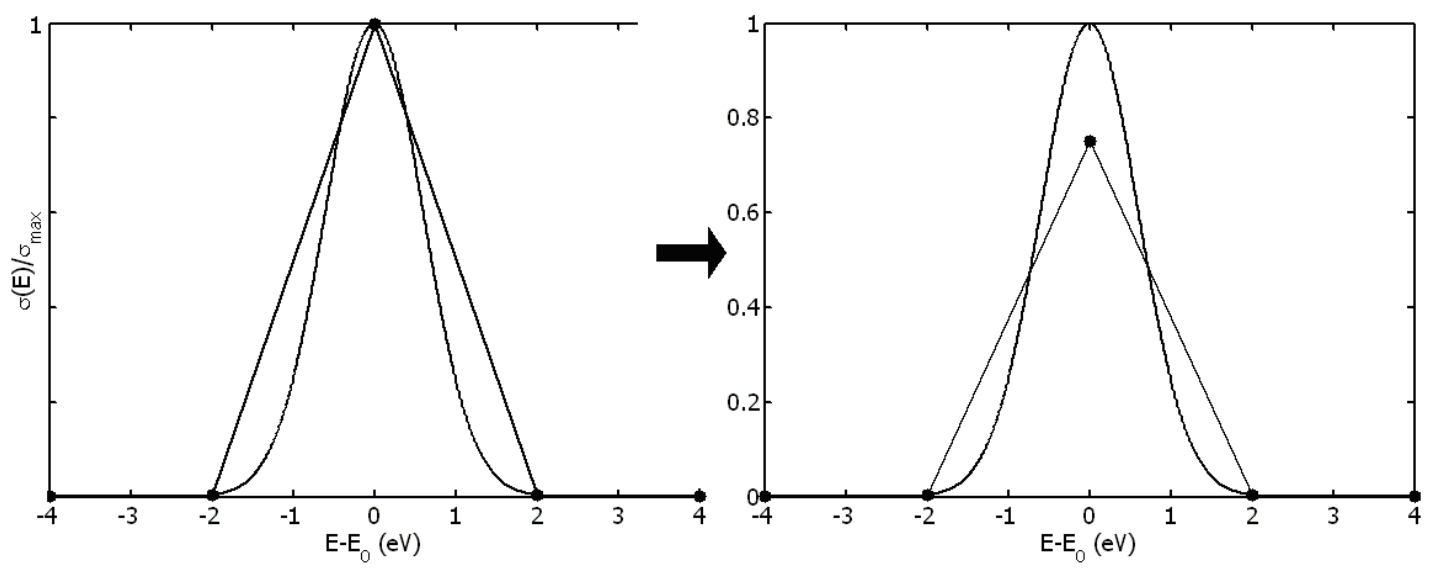

Figure E.1 Point-wise discretization of Maxwell-Boltzmann cross section, renormalized to preserve integrated cross section.

The resulting array, TOTXS, is two columns wide, with each row containing an energy in the first column and the corresponding computed NRF cross section in the second. Each level in the DATASET array results in five rows in the TOTXS array such that each NRF peak is fitted by five points. In the above formulations, it is assumed that the resonances can be well-represented as isolated from one another.

\section{E.1.6 NRFXSSI Step 4: $\gamma$-ray Cascade Analysis}

The subroutine CASCADECALC.CPP processes the $\gamma$-ray cascades emitted from each NRF level. It does this by identifying the levels in CASCADE that correspond to NRF levels identified in DATASET. With the level identified, CASCADECALC.CPP calls a subroutine CASCADEGRAB that loops over each $\gamma$ ray emitted from the level, finds the probability of emission for each $\gamma$ ray, and, if necessary, calls itself to analyze the daughter levels populated by $\gamma$-ray emissions from the 
level. The net result is a new array named GAMARRAYPRELIM. The entries of the columns of GAMARRAYPRELIM are the following:

0. Energy of the NRF level. (All energies are expressed in eV.)

1. Energy of the level that emitted the $\gamma$ ray.

2. Energy of the $\gamma$ ray.

3. Number of $\gamma$ rays emitted per excitation of the level.

4. Level number (LN) of the $\gamma$-ray emitting level. This is subsequently changed to the total number of discrete $\gamma$-ray energies that can be emitted from the corresponding NRF level.

5. Spin of the $\gamma$-ray-emitting state.

6. LN of the daughter $\gamma$-ray level. This subsequently becomes the spin of the ground state or -1 .

After CASCADEGRAB is complete, GAMARRAYPRELIM is sorted. $\gamma$ rays emitted from a given NRF level are placed in ascending order and redundant $\gamma$-ray lines are combined, with their respective probabilities summed.

After this sorting process, LN in Column 4 of GAMARRAYPRELIM is replaced with the number of $\gamma$ rays emitted from a given NRF level and the daughter LN in Column 6 is replaced by the spin of the daughter level, or -1 to denote that the state populated by the $\gamma$-ray emission is the ground state.

With GAMARRAYPRELIM complete, CASCADECALC.CPP computes the $\gamma$-ray multiplicity of each NRF level. The multiplicity of a level is defined as the mean number of emitted $\gamma$ rays per excitation of the NRF level, which is the sum of the values in Column 3 of the GAMARRAYPRELIM array corresponding to a single NRF level. The multiplicities are stored in the Column 1 of the array GMULTS, and the corresponding energy of each level is stored in Column 0. GMULTS also has a header row which contains the total number of resonances in both entries in the row.

Finally, CASCADECALC.CPP produces the array, GAMARRAY. The values of the rows of GAMARRAY are nearly identical to those of GAMARRAYPRELIM. The primary difference is that GAMARRAY is formatted to be entered into the ENDF format as a point-wise evaluation of the multiplicity versus energy function. It is assumed that outside of the resonance energies, the multiplicity for $\gamma$-ray emission is zero. Because of this, each line in GAMARRAYPRELIM becomes 3 lines in GAMARRAY. The first of the three lines corresponds to the lower energy bound of possible $\gamma$-ray energies emitted during a specific de-excitation of the state. The entry in Column 2 (the energy of this data point) is $10 \mathrm{eV}$ less than the energy of the $\gamma$ ray. The corresponding yield, Column 3 , is set to 0 . The second row is identical to the corresponding GAMARRAYPRELIM row, except that the yield must be re-normalized such that the integral over the multiplicity function results in the yield computed for GAMARRAYPRELIM (Column 3), and the $\gamma$-ray multiplicity in GMULTS is factored out. This value is $Y_{i} / 10 / M_{i}$, where $Y_{i}$ is the corresponding value in Column 3 of GAMMARRAYPRELIM, and $M_{i}$ is the corresponding value in Column 1 of GMults. The number, 10, is one-half the width of the multiplicity function which is integrated in radiation transport codes to determine yield. The third row for a single $\gamma$ ray in GAMARRAY is identical to the first row, 
except that the energy is now $10 \mathrm{eV}$ above the nominal energy of the $\gamma$ ray - the upper bound for the yield distribution function. GAMARRAY is passed to INSERT.CPP, which formats the data and inserts it into the MT6 $\mathrm{MF}^{4}$ section of the resulting ENDF file.

\section{E.1.7 NRFXSSI Step 5: Data Formatting and Integration}

NEW355.CPP is called to combine NRF cross section data (produced in NRFCALC.CPP) with cross section data that may be present in the photonuclear ENDF datafile that already exists for the isotope. The data in MT3 in an ENDF file is the cross section data. MF3 corresponds to the total cross section and MF5 corresponds to cross section data for "other types of reactions", which NRF is considered. The subroutine reads the cross section data in the old ENDF file, and inserts the NRF cross sections from the TOTXS array into the appropriate location. In the event that the preexisting cross section is non-zero at an energy where TOTXS is defined, values of the pre-existing cross section are linearly interpolated to the energies where the NRF cross sections are defined and the resulting total cross section is determined by adding these values to the NRF cross section. The resulting arrays, A33 and A35 are then formatted to be inserted into the ENDF photonuclear file.

\section{E.1.8 NRFXSSI Step 6: Data Insertion into Photonuclear ENDF File}

The final step of NRFXSSI is to insert the NRF data into the a photonuclear ENDF file. The subroutine INSERT.CPP accomplishes this. INSERT opens the photonuclear ENDF file, updates the file's descriptor block, and inserts NRF cross section and secondary $\gamma$-ray emission data along with the proper header information.

The first step is to read the data already present in the ENDF file and to modify the file descriptor section (MT1 MF451). All information is read from the input file and either copied or modified as it is written to a temporary output file, TEMP.ENDF. The changes to the descriptor section include incrementing by 1 NMOD, an integer describing how many modifications this ENDF file has undergone, adding a text boilerplate that describes the changes to the ENDF file, checking that there are MT3 MF3, MT3 MF5, and MT6 MF5 section directories in the description section, and either deleting the entries for the lengths of these sections, which will later be updated, or making directory place-holders for these sections to be updated later.

After the descriptor section is modified, INSERT skips forward to the section MT3 MF3, deletes all old data and inserts the array, A33, along with the proper header information. The subroutine NRFIMP.CPP is called to perform the insertion. After MT3 MF3, the MT3 MF5 section is modified using identical logic as the MT3 MF3 section except that the array A35 is used as the data source. The MT6 MF5 insertion involves slightly different formatting, and is accomplished by inserting the GMULTS multiplicity data, and GAMARRAY secondary photon data. Similar logic to the NRFIMP.CPP subroutine is present in this section of INSERT.

Finally, INSERT rewinds TEMP.ENDF and opens the final output file, whose name is identical to the original input file, but is located in the folder/OUT/. TEMP.ENDF is copied verbatim to the output file, but the lengths (in number of rows) of the newly inserted or modified sections are added to the file directory in the file descriptor section.

\footnotetext{
${ }^{4}$ See reference[59] for descriptions of the data formats used in ENDF files.
} 


\section{E.1.9 ACE file Creation using NJOY}

With ENDF files created, the data processing code NJOY was used to convert the ENDF files to ACE files. NJOY was called by the following command: NJOY $259<$ IN.DAT. An example of IN.DAT for processing the ${ }^{238} \mathrm{U}$ ENDF file is shown below:

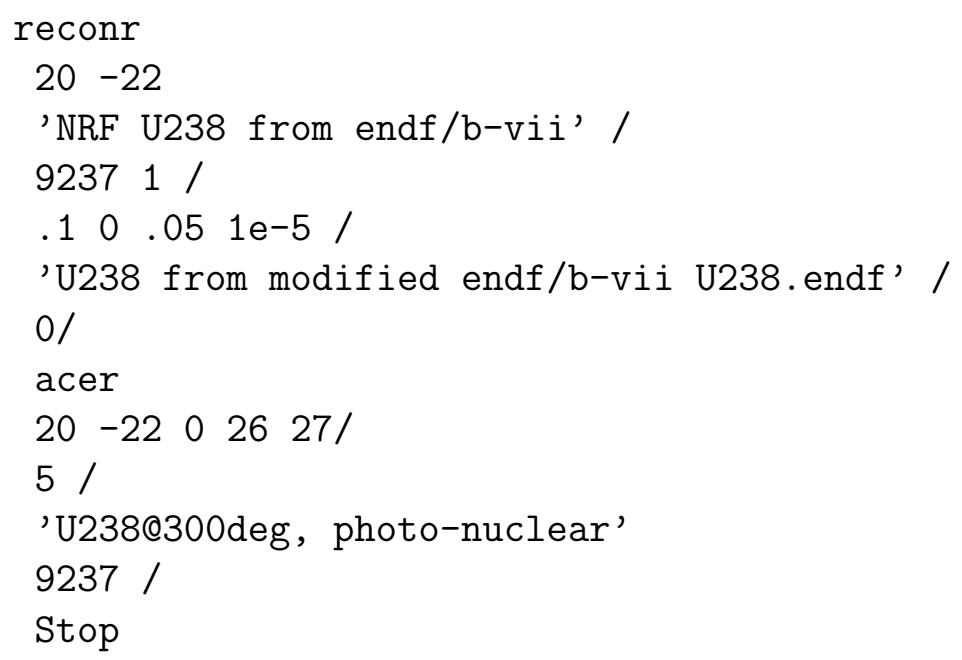

NJOY assumes all input and output files are "tapes". To use the above example, the ${ }^{238} \mathrm{U}$ ENDF file output from NRFXSSI must first be copied to / NJOY/TAPE20. Likewise, the outputs of NJOY are the files TAPE26 and TAPE27. TAPE26 is an ACE file and TAPE27 is the XSDIR line corresponding to the ACE file. The number 9237 identifies ${ }^{238} \mathrm{U}$, and the ' 5 ' in the second line of the ACER call tells NJOY that the ENDF data is photonuclear.

\section{E.1.10 NJOY Bug Repair}

NJOY was found to have a simple systematic bug when processing the photonuclear ENDF files produced by NRFXSSI. Upon completing an NJOY run, the angular distribution locators for secondary particles (the LANDP array) in the output ACE file are incorrectly defined. The error was found to be systematic. The first entry of LANDP should always be 1, but instead, the correct value for the second entry is inserted. Similar problems continue for the remaining entries. The code TAPE26FIX.EXE was written to fix the LANDP array. The output of TAPE26FIX is a properly formatted photonuclear ACE file that may be used in MCNPX.

\section{E.1.11 NRF data insertion results}

Figure E.2 provides an example of a NRF cross section read by MCNPX, and displayed using the cross section plotting functionality in the code.

Figures E.3 and E.4 demonstrate a spectrum that was calculated using the new photonuclear datafiles and the modification of the MCNPX code described in Appendix D. In this simulation, a pencil beam of photons, uniformly intense between 2.430 and $2.432 \mathrm{MeV}$, irradiated a cylinder of ${ }^{239} \mathrm{Pu}$ with $0.5 \mathrm{~cm}$ diameter and $1 \mathrm{~cm}$ length. The photon flux at a distance $60 \mathrm{~cm}$ from the cylinder center was calculated at an angle of $135^{\circ}$ relative to the pencil beam direction using the next-event-estimator tally (see Section F.3). The spectrum demonstrates several features. At energies below $2.4 \mathrm{MeV}$, the spectrum is dominated by the inelastic scattering processes. These 


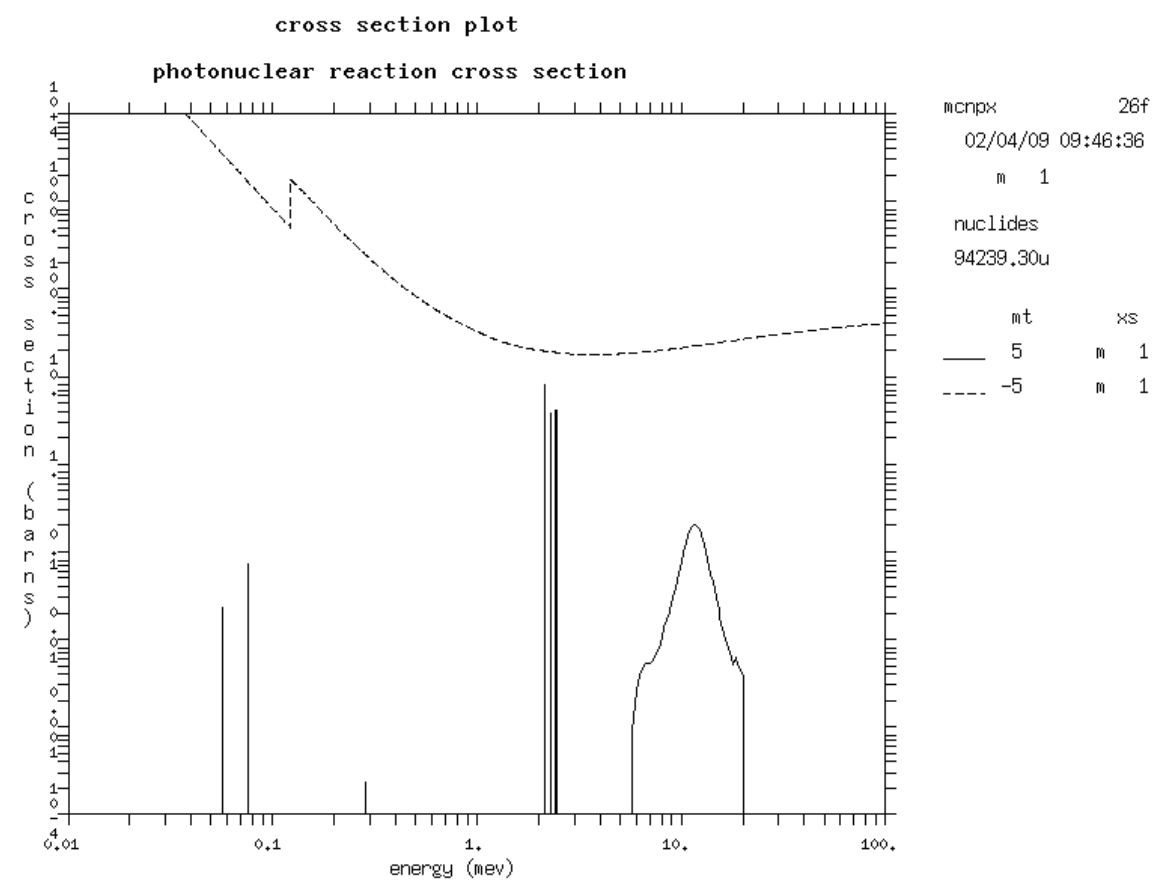

Figure E.2 The ${ }^{239} \mathrm{Pu}$ cross photon sections as read by MCNPX. The dashed line indicates the photo-atomic cross section, and the solid line indicates the photonuclear cross section. Before this modification, the photonuclear cross section file only contained the feature in the 6 to $20 \mathrm{MeV}$ range.

processes result in an annihilation peak at $511 \mathrm{keV}$, a Compton-scatter photo-peak at $266 \mathrm{keV}$, and a continuum due to photo-electron bremsstrahlung production. At the initial beam energy, elastic scatter of photons produces a profile that is similar to the beam profile. This feature is only present because of the modifications to the code described in Appendix D. Finally, two additional peaks are visible at 2431.7 and $2423.8 \mathrm{keV}$. These are due to resonance fluorescence of the $2431 \mathrm{keV}$ state in ${ }^{239} \mathrm{Pu}$, which de-excites to the ground and first-excited states by $\gamma$-ray emission, resulting in two NRF $\gamma$ rays separated in energy by $7.9 \mathrm{keV}$. 


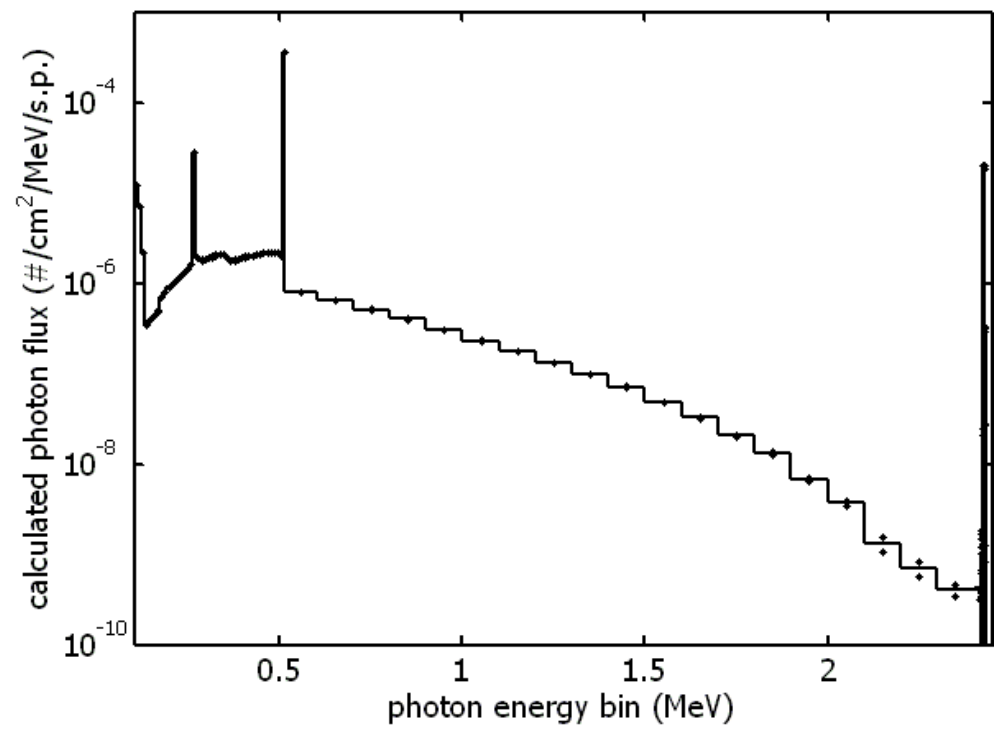

Figure E.3 MCNPX-calculated backscattered photon spectrum per simulated photon due to $2431 \pm 1 \mathrm{keV}$ photons incident upon ${ }^{239} \mathrm{Pu}$. Statistical uncertainties are shown as dots above and below the center of each energy bin.

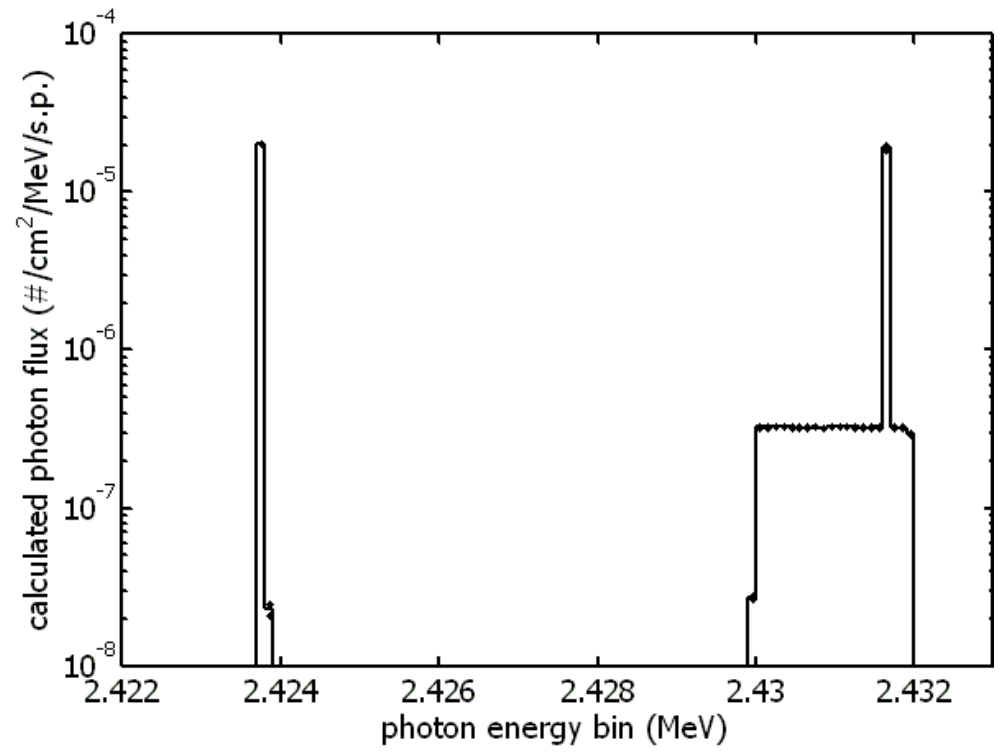

Figure E.4 Same as Figure E.3, except the energy range between 2422 and $2433 \mathrm{keV}$ is expanded. 


\section{F Supporting Documentation of MCNPX Simulations}

Throughout this report, results of MCNPX simulations have been presented. This appendix describes the logic behind some of the more complicated simulations and presents example input files. The primary cause of complications is the use of variance reduction techniques. Variance reduction is defined as using methods to reduce the statistical uncertainty (or variance) of a simulation result in the same amount of time. There are three primary types of variance reduction. The first is to truncate a problem in either geometry or energy space such that irrelevant portions of the simulation-space are not simulated. This is essentially done in all simulations since no model may contain all details of the physical world.

In MCNPX, variance reduction may also take the form of non-physical adjustment of the number of particles simulated and correspondingly modifying the weights of these particles. For example, the simulation described in Section F.3 calculates the photon flux backscattered from a target. Photons that are incident upon the target volume are forced to undergo an interaction, which would normally provide non-physical results. To keep the results physical, MCNPX does the following. First, the probability, $p_{\text {int }}$, for the incident photon to interact along its path through the TD is computed. The location of the forced interaction along the photon's path is then randomly sampled, and a new particle is created at that location with weight, $p_{\text {int }}$ relative to the weight, $w$ of the incident photon. This photon is forced to undergo some collision, whereas the fate of the initial photon continues and is then simulated without any modification, except that its weight is reduced to $w\left(1-p_{\text {int }}\right)$. The effect of this weight-adjusting variance reduction technique is to promote the simulation of particles that have interacted in the target volume, which, for this simulation, results in reducing the statistical uncertainty of the calculated flux.

Weight-varying methods may also be implemented during definition of the probability of generating particles during the simulation (see Section F.1), by increasing the probability that a simulated particle is transmitted through a material, or by inducing extra particles to be simulated in portions of the geometry that are deemed important (see Section F.2).

A third type of variance reduction method involves next-event estimators. Next-event estimators essentially add deterministic treatment to otherwise stochastic modeling. A point or region of interest is defined and each time a particle interacts within the simulation, the expected contribution of the flux at this point is deterministically calculated. This method works quite well when the majority of interactions occur sufficiently far from the point of interest and the angular distribution of particles emitted due to the interaction event are known. Next-event estimators are used in the backscattered photon flux calculations described in Section F.3. Finally, details concerning the treatment of electrons and the production of bremsstrahlung by MCNPX are provided in Sections F.5 and F.6.

\section{F.1 Bremsstrahlung Source Simulations}

Bremsstrahlung was assumed to be the photon source for many of the simulated NRF experiments described in this report. These spectra were calculated using MCNPX with input files similar to the one provided below. The simulated geometry is monoenergetic electrons normally incident 
upon a $102 \mu \mathrm{m}$ thick gold converter $(\mathrm{Z}=79)$ that is supported by $1 \mathrm{~cm}$ thick copper $(\mathrm{Z}=26)$ slab. There is no shielding simulated. In the simulation, the energy and relative angle of photons that cross the surface of a $50 \mathrm{~cm}$ radius sphere whose center is the intersection of the electron beam and the gold foil (surface 10) are counted. The lines phys:e 2.9 8j 1 and bbrem 1. $1.46 \mathrm{I} 2.01$ are of primary interest. The first line indicates that the electron data library need only extend up to $2.9 \mathrm{MeV}$, that all electron physics processes are default except that bremsstrahlung photon production is biased such that one reduced-weight photon is produced at each electron substep rather than probabilistic sampling of full-weight photon production. The second line indicates that bremsstrahlung photon production in material 1 (gold) is to be biased such that higher energy photons are more likely to be produced at correspondingly reduced weight.

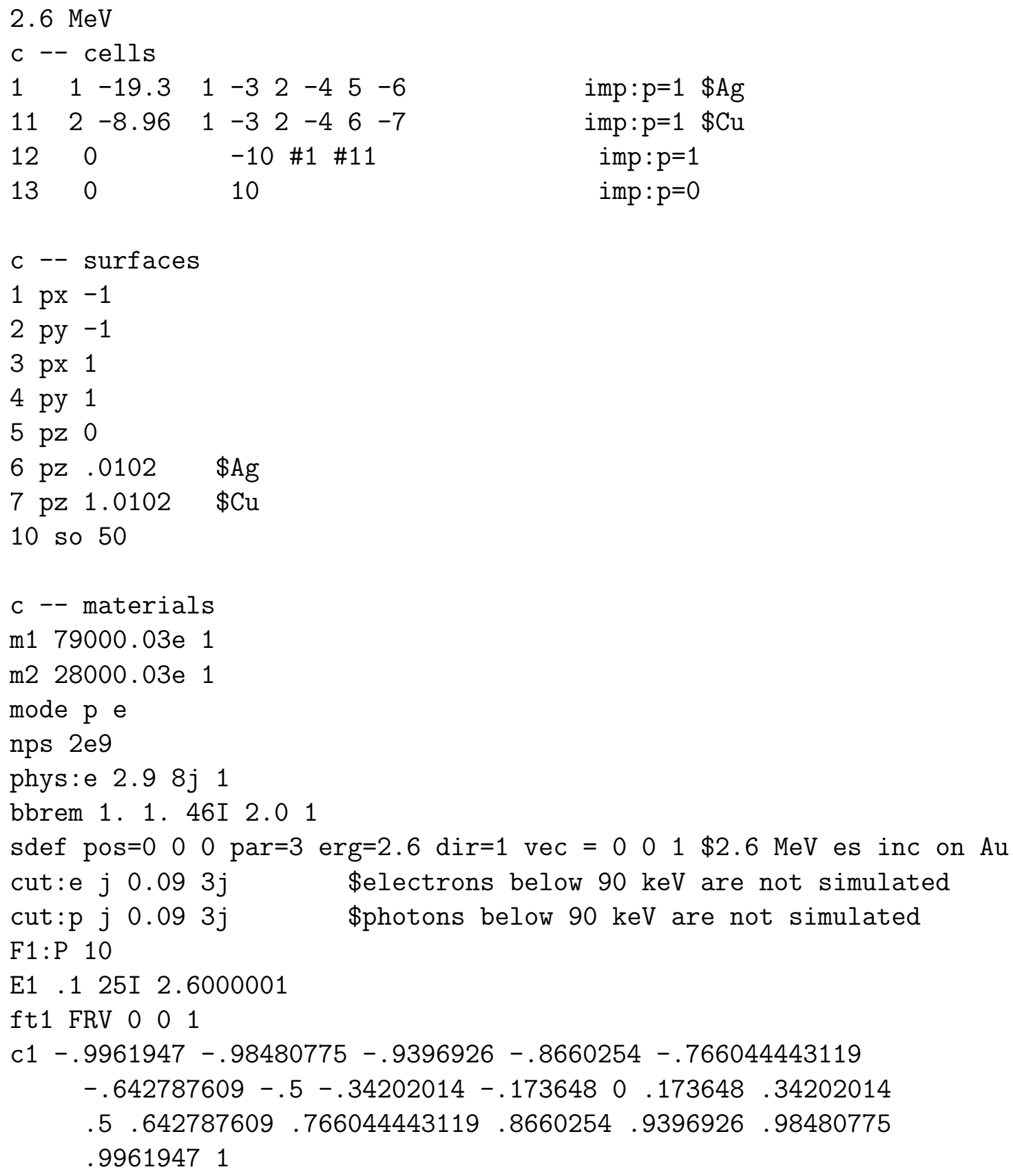




\section{F.2 Photon Penetration Through Thick Shielding}

Shielding calculations tend to be difficult to simulate with Monte Carlo radiation transport codes. For example, the probabilities that a $0.5,1$, and $2 \mathrm{MeV}$ photon penetrates $25 \mathrm{~cm}$ of $\mathrm{Pb}$ without interacting are $3.4 \times 10^{-19}, 4.2 \times 10^{-9}$, and $2.1 \times 10^{-6}$, respectively. To calculate shielding requirements for a bremsstrahlung spectrum, variance reduction techniques must be used to avoid using unnecessary computation time simulating particles that will never reach the opposite side of the shield.

The variance reduction technique uses the following routine.

- The simulation is conducted with no variance reduction, but with reduced shielding density. Weight-windows are generated.

- The density of the shielding is increased and the simulation is re-run with variance reduction. New weight-windows are regenerated.

- This process continues until the full shielding density is simulated.

Use of weight windows is a particle-weight-modifying variance reduction technique. Weight windows divide the geometry of a simulation into segments, and each segment is assigned weight cutoffs. If a simulated particle enters a segment with a weight greater than the segment's upper weight cutoff, the particle is divided into multiple particles of reduced weight, which are then separately simulated. If a simulated particle enters a segment with a weight below the segment's lower weight cutoff the particle is subjected to termination (also called Russian Rouletting), with probability, $p_{R R}$. If the particle is not terminated, the weight of the particle is increased by $1 / p_{R R}$. Likewise, weight-window segments can be generated for different energy ranges.

To generate weight windows, the WWG and WWGE (for energy segmentation) cards are used in the MCNPX input files. These cards cause the code to determine from which segments, and at which energies, came particles that contribute to the flux (or an energy range of the entire flux) that is specified to be important. If a segment is found to be important, the weight cutoffs are lowered, causing more particles with reduced weight be generated in the segment. After the weight window-generating simulation, the weight-windows are written to a file, which may be read for subsequent simulation. The method of iteratively increasing the shielding material density and creating a series of weight-window files allows simulated particles to contribute to the flux with higher probability (and reduced weights), which subsequently allows the code to more efficiently determine how to weight the segments.

A second variance reduction method was also employed to increase the probability that simulated photons would penetrate the shielding. This involved the exponential transform function, invoked by the EXT card. The exponential transform modifies probability distribution function (PDF) that describes the distance to collision, $x_{c}$. Normally, this PDF is given by $P(x)=N \sigma \exp (-N \sigma x)$ where $N$ is the number density of atoms in the material and $\sigma$ is the total reaction cross section. This PDF may be sampled using a random number ${ }^{1}, r$, to obtain $x_{c}=\frac{-\ln (r)}{N \sigma}$. The exponential transform creates a new non-physical cross section, $\sigma^{*}$, samples $x_{c}$ using this value, and if the particle interacts within the transform cell, it increases the weight of

\footnotetext{
${ }^{1}$ See Section D.2 for a description of PDF sampling.
} 
the particle by

$$
w=\frac{\sigma \exp \left(-N \sigma x_{c}\right)}{\sigma^{*} \exp \left(-N \sigma^{*} x_{c}\right)}
$$

If the particle does not react, its weight is reduced by

$$
w=\frac{\exp \left(-N \sigma x_{c}\right)}{\exp \left(-N \sigma^{*} x_{c}\right)}
$$

This conserves the probability for particle transport by correctly increasing interacting particle weights and reducing weights for transmitted particles.

The draw-back of the exponential transform is that it may under-sample scattering events. Because of this, the statistical analyses of the Monte Carlo simulation are carefully considered and the value of $\sigma^{*} / \sigma$ is taken to be as close to unity as is practical.

An example of an input file for a MCNPX shielding simulation is shown below. The simulation is one of the series to determine necessary shielding thicknesses between a bremsstrahlung converter and an HPGe detector. This particular file is the last of four iterations simulating the penetration of the $40-50^{\circ}$ angular range of a $2.5 \mathrm{MeV}$ endpoint-energy bremsstrahlung beam through $40 \mathrm{~cm}$ of tungsten.

$40 \mathrm{~cm} \mathrm{~W}$ shield, $2.5 \mathrm{MeV}$ endpoint-E photons rho should be $19.3 \mathrm{~g} / \mathrm{cm} 3$

c -- cells

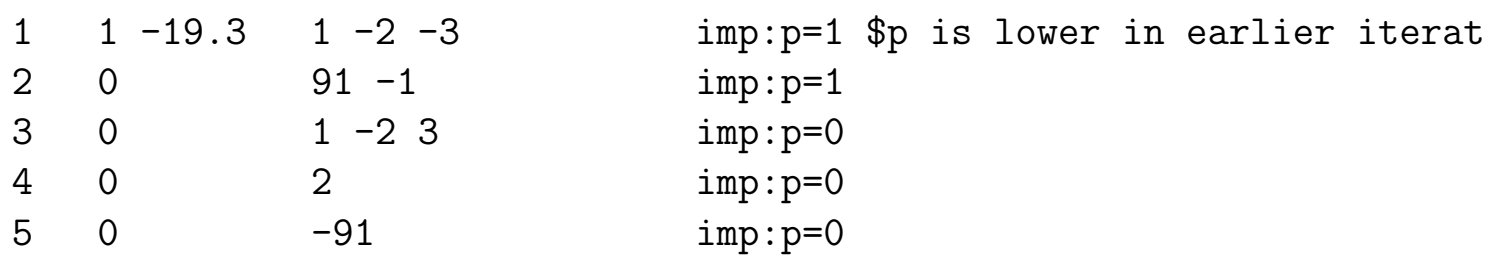

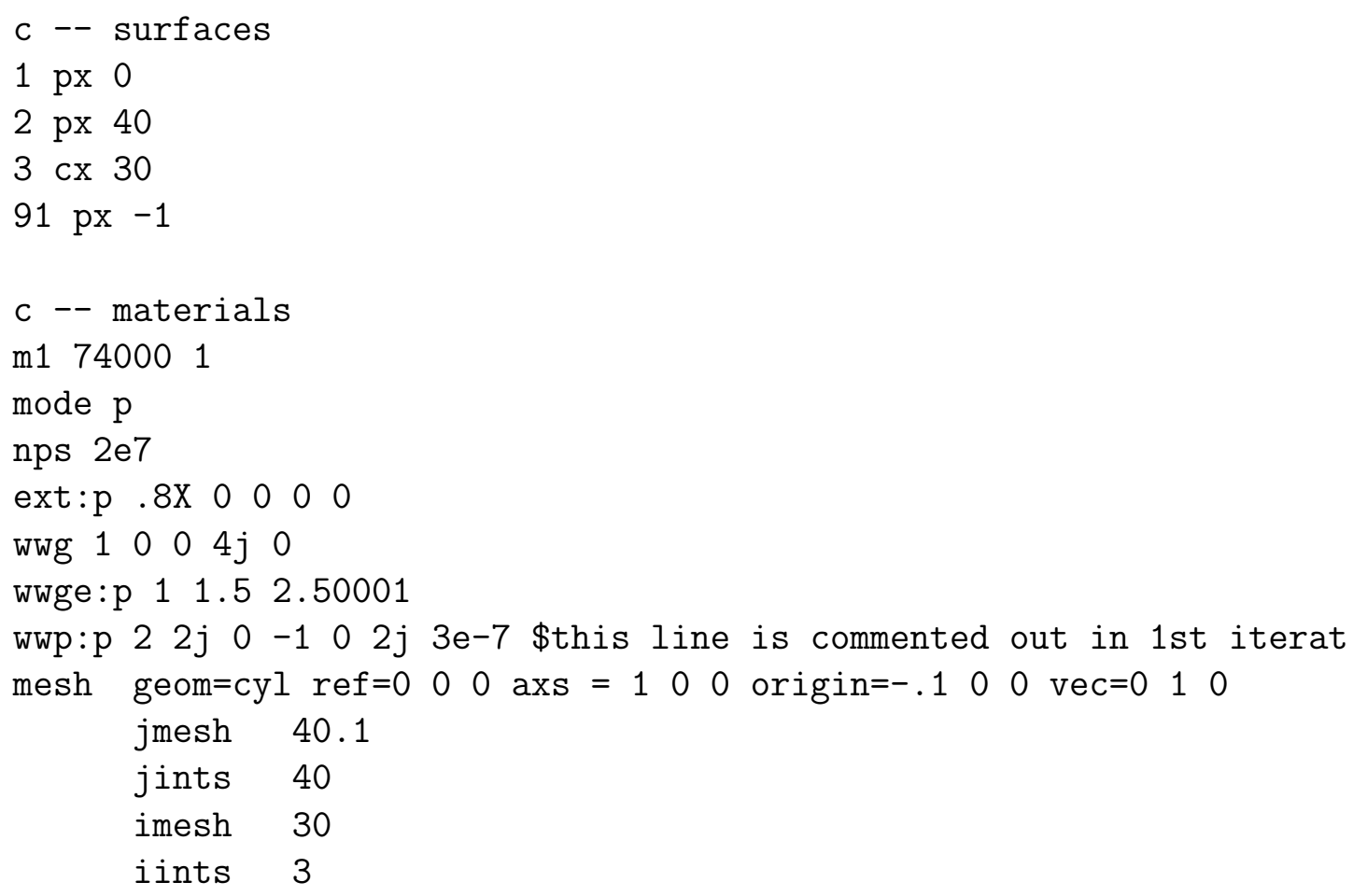




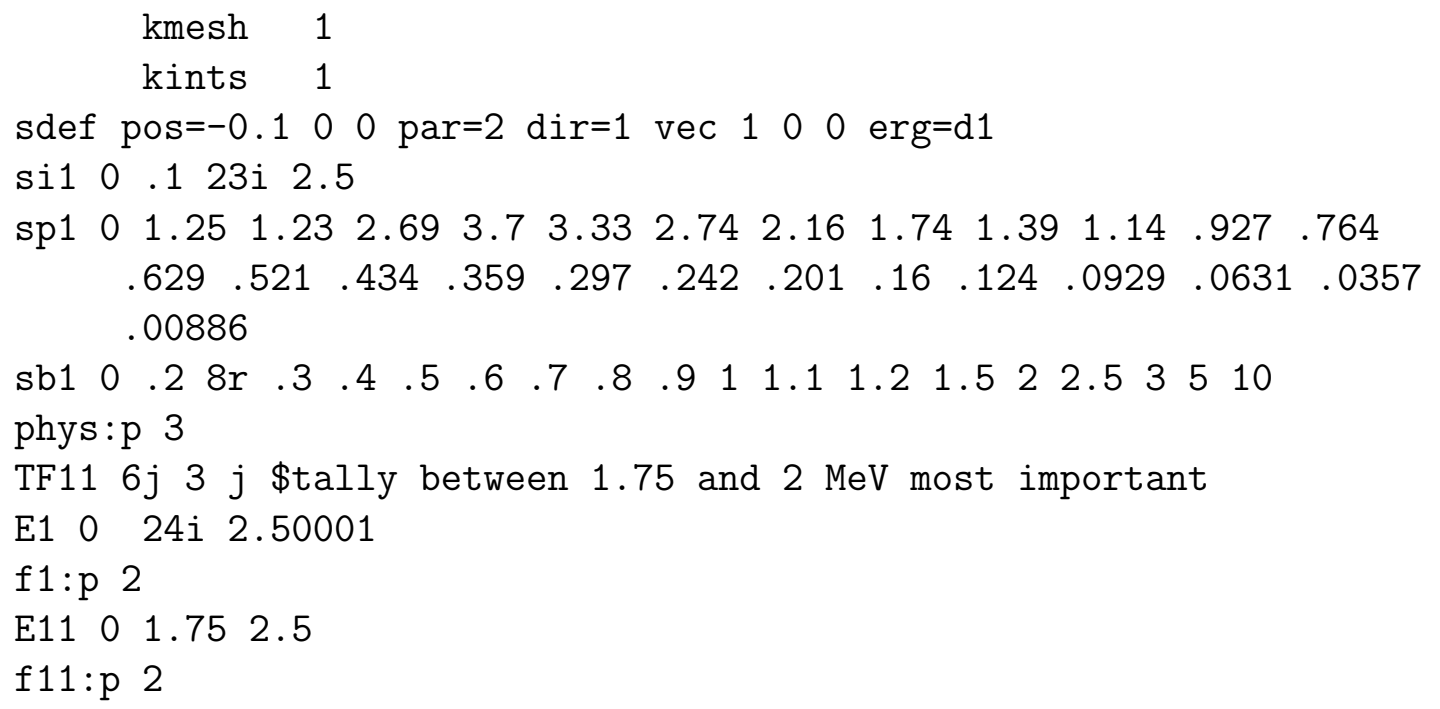

\section{F.2.1 Comparison to Published Build-up Factors}

The effect of down-scattering has been approached by modifying simple attenuation by use of a build-up factor, $B(Z, E, \mu x)$, that is a function of energy, material composition, and geometry. This results in an effective attenuation expression that is written as

$$
I=I_{o} B(Z, E, \mu x) \exp (-\mu(Z, E) x)
$$

There are several different formulations of build-up factors. The factors most commonly encountered apply to dose-rates rather than fluxes.

One build-up formulation is the polynomial form:

$$
B(Z, E, \mu x)=1+a(Z, E) \times(\mu x)+b(Z, E) \times(\mu x)^{2}+c(Z, E) \times(\mu x)^{3}
$$

Values of $a, b$, and $c$ for energy transmission through shielding from an isotropic point photon source were taken from reference [60], and used to find an expected build-up factor. $B(Z, E, \mu x)=$ 23.42 for $4 \mathrm{MeV}$ photons, emitted from the center of a $30 \mathrm{~cm}$ radius tungsten shield. Using equation F.3 and an attenuation coefficient, $\mu=0.779 \mathrm{~cm}^{-1}[49]$, we expect each photon to transmit, on average the following energy:

$$
\begin{aligned}
E=E_{\gamma} B \exp (-\mu x) & \\
& =23.42 \times 4[\mathrm{MeV}] \times \exp \left(-30[\mathrm{~cm}] \times 0.779\left[\mathrm{~cm}^{-1}\right]\right)=6.60 \times 10^{-9}[\mathrm{MeV}]
\end{aligned}
$$

The energy build-up formalism is useful for estimating the energy or dose due to a photon fluence penetrating shielding, but it does not provide an estimate of the number of individual photons that escape the shielding. This renders these build-up factors useless for predicting detector responses, to shielded photon fluxes when detectors are operated in pulse mode ${ }^{2}$. To make these predictions, we turn to Monte Carlo calculations using MCNPX and built-in variance reduction functionality, while using the published build-up parameters as a check on our use of the code.

\footnotetext{
${ }^{2}$ The converse is true, if detectors are operated in an integrating mode, where the charge deposited in a detector is continuously integrated by a capacitive circuit.
} 


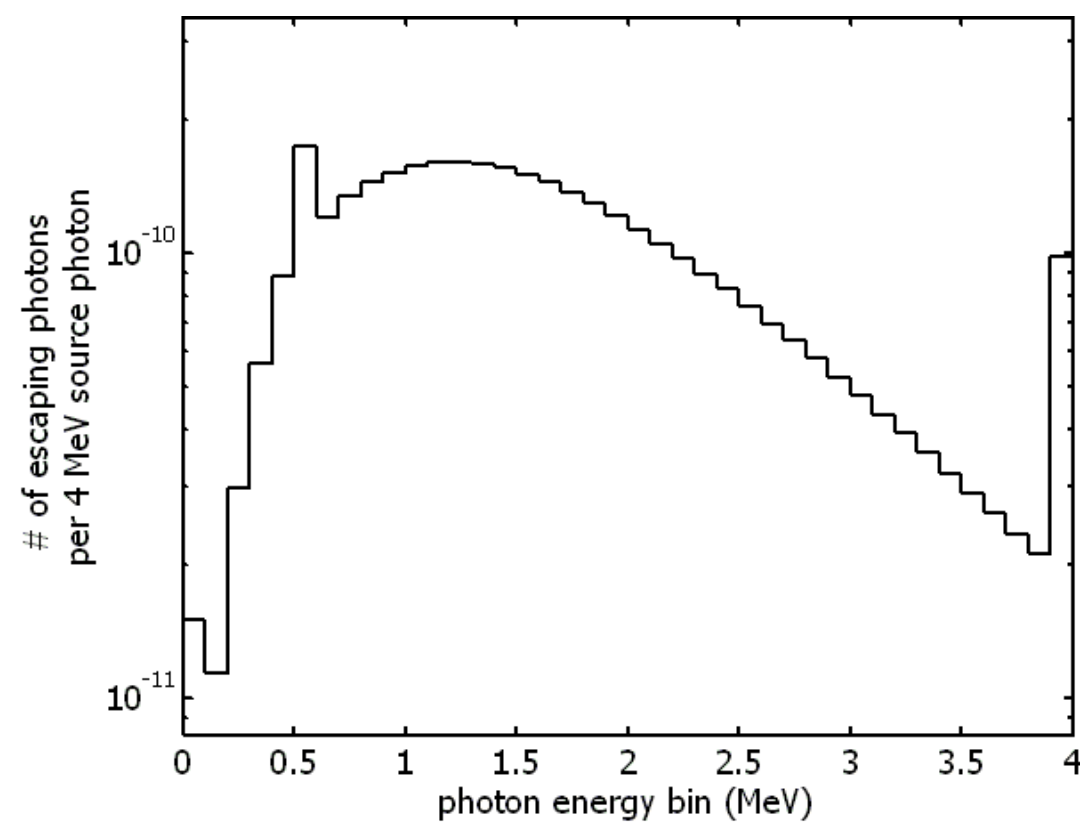

Figure F.1 MCNPX-calculated photon spectrum leaving tungsten shielding for a $4 \mathrm{MeV}$ point isotropic source located at the center of a tungsten sphere of $30 \mathrm{~cm}$ radius. Statistical errors are negligible.

To check the validity of the MCNPX results, we first simulate a geometry identical to that whose build-up constants are tabulated; a point isotropic $4 \mathrm{MeV}$ photon source located in the center of a $30 \mathrm{~cm}$ radius $\mathrm{W}$ sphere. The calculated energy spectrum of photons emitted from the tungsten shield is shown in Figure F.1. To convert this spectrum into emitted energy, and subsequently, a build-up value, probability of photon emission is multiplied by the corresponding energy, and summed for the entire spectrum. This calculation results in the computed average energy leaving the tungsten sphere per $4 \mathrm{MeV}$ photon emitted from the sphere's center, which was $6.14 \times 10^{-9} \mathrm{MeV}$ per $4 \mathrm{MeV}$ source photon for the MCNPX computation. The corresponding energy build-up factor is $B_{\mathrm{MCNPX}}=21.8$. The agreement between this value and that from equation F.5 is satisfactory, given that differing photon cross section data were used for the two computations. We thus conclude that using variance reduction techniques enables MCNPX to provide reasonable estimates of build-up factors, suggesting that the MCNPX calculations are likely to be accurate for studies of photon fluences penetrating through shielding.

\section{F.2.2 Shielding Calculations for Reduction of Source Bremsstrahlung Flux}

To reduce direct detection of interrogation photons, large quantities of shielding are generally placed between the photon source and the radiation detectors. High- $Z$ materials such as tungsten and lead have high densities and relative to lower- $Z$ materials, have larger pair production and photoelectric absorption cross sections, compared to their incoherent scattering cross sections. However, incoherent scattering is still frequently the dominant process for multi-MeV photons. Incoherent scattering tends to produce forward-scattered photons with energies near the un-scattered photon energy, which implies that incoherent scatter is a relatively ineffective process for shielding, relative to other photon interactions. Therefore, simple exponential attenuation using the total interaction cross section, can significantly underestimate the transmitted photon flux. 


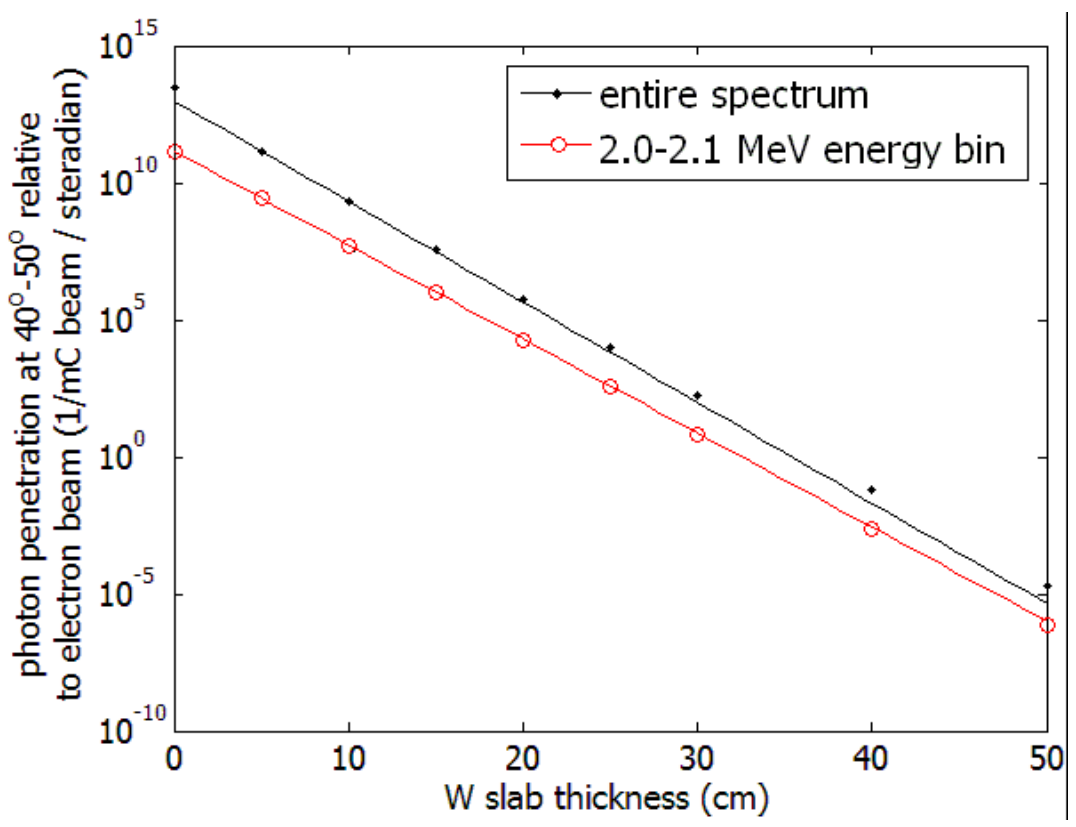

Figure F.2 MCNPX-calculated photon intensities transmitted through a tungsten slab of varying thickness. The open and closed circles indicate calculation results and the solid line indicates exponential fits to the calculated results. The photon spectrum incident upon the shield is that of the $40^{\circ}-50^{\circ}$ angular bin bremsstrahlung beam created by impinging $2.5 \mathrm{MeV}$ electrons upon a $1 \mathrm{~mm}$ thick slab of uranium. Statistical errors are not shown, but the largest error fraction is $5.3 \%$ of the calculated value for the $2-2.1 \mathrm{MeV}$ bin penetrating through $40 \mathrm{~cm}$ of tungsten.

MCNPX simulations were performed to determine the transmission of bremsstrahlung-spectrum photons through large quantities of shielding. The simulated geometry was assumed to be a slab of tungsten whose thickness was varied between simulations. The photon spectrum incident upon the shielding slab was taken from the results of a previous MCNPX simulation in which $2.5 \mathrm{MeV}$ electrons irradiated a $1 \mathrm{~mm}$ thick sheet of uranium. Photons emitted from the uranium converter within a bin of $40^{\circ}-50^{\circ}$ from the initial electron trajectory were used as the photon source for the shielding penetration simulation. Of interest are both total photon intensities and the intensity of photons with near-resonant energies that would provide background to observing NRF $\gamma$ rays. Figure F.2 presents intensities of photons after the bremsstrahlung-spectrum photons penetrate through tungsten shielding. As an example of near-resonant photons, the photon intensity for energies between 2 and $2.1 \mathrm{MeV}$ is shown along with the full energy-integrated spectrum. The spectra have been normalized to number of photons per steradian per $\mathrm{mC}$ of electron charge incident upon the bremsstrahlung converter.

The rates at which $2-2.1 \mathrm{MeV}$ photons and the entire spectrum are attenuated both appear to follow exponential forms for thicker shields. Linear least-squares routines were applied to the logarithms of the attenuation values, yielding a function of the form $\left(I / I_{0}\right)_{\text {fit }}=a \exp (-b x)$. The results of these routines are shown in Table F.1. When fitting the attenuation of the entire spectrum, the least-squares fitting routine was only applied to simulated values of photon transmission for $\mathrm{W}$ shielding thicknesses greater than $5 \mathrm{~cm}$. The fits are compared to the calculated attenuations in Figure F.3, where values of $\frac{\left(I / I_{0}\right)_{\mathrm{MCNPX}}-\left(I / I_{0}\right)_{\mathrm{fit}}}{\left(I / I_{0}\right)_{\mathrm{MCNPX}}}$ are plotted versus tungsten slab thicknesses.

In an ideal NRF geometry, the bremsstrahlung flux intensity, $d \Phi / d E$, incident upon a detector that has penetrated the bremsstrahlung shielding is significantly lower than that due to NRF. 
Table F.1 Fit parameters for representing bremsstrahlung-spectrum photon intensities as $\left(I / I_{0}\right)_{\text {fit }}=a \exp (-b x)$ after attenuation through a thickness, $x$ of shielding.

\begin{tabular}{rcc}
\hline \hline energy range & $a$ & $b\left(\mathrm{~cm}^{-1}\right)$ \\
\hline entire spectrum & $0.3275 \pm 6.4 \times 10^{-4}$ & $0.845 \pm 0.0004$ \\
$2-2.1 \mathrm{MeV}$ & $4.737 \times 10^{-3} \pm 4 \times 10^{-8}$ & $0.7898 \pm 0.0001$ \\
\hline
\end{tabular}

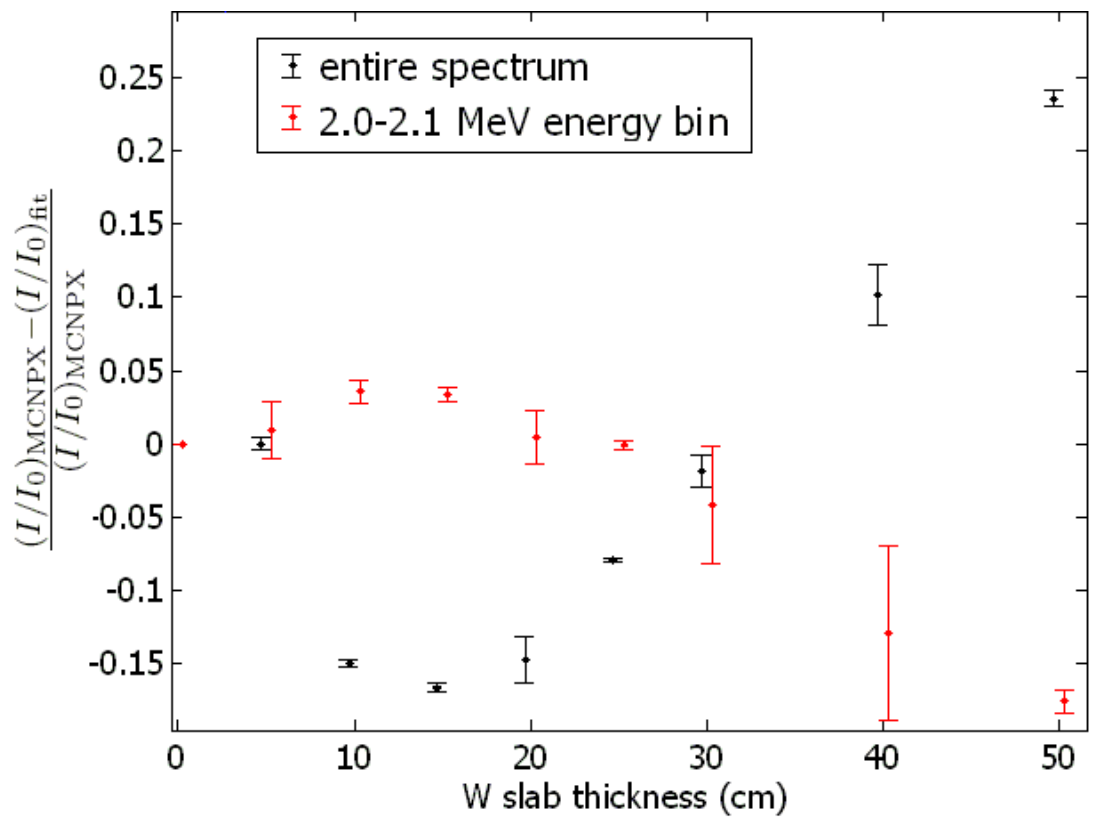

Figure F.3 Fractional deviation between the calculated values of the transmitted photon intensity and those estimated by a linear least-squares fitting-routine. The error bars indicate $\pm 1 \sigma$ errors from the MCNPX computation. The entire spectrum value is not shown for zero slab thickness, it is 0.72 . 
Likewise, the energy-integrated flux must be sufficiently small to permit detector operation at reasonable count rates.

\section{F.3 Backscattered Photon Flux Calculations}

Calculations of backscattered photon spectra and intensities is an important part of predicting the relative strengths of the NRF $\gamma$ rays and background photons. The simulations cannot be accurately performed without variance reduction because the fraction of photon scattering events that result in large-angle scatter without significant energy loss is very small. For example, if a $2.4 \mathrm{MeV}$ photon is incident upon a $1 \mathrm{~cm}$ thick slab of uranium, it will interact with a probability of approximately 0.6 . Of the fraction of photons leaving $1 \mathrm{~cm}$ of uranium due to scattering, approximately one-fifth are in the backwards direction, and the majority of these are low-energy. Of the entire backscattered intensity, approximately $10^{-3}$ are elastically scattered, and approximately $2 \times 10^{-4}$ are inelastically down-scattered to an energy between 2.1 and $2.2 \mathrm{MeV}$. Because of these low probabilities, direct simulation of backscattered photon intensities near the endpoint energy of a bremsstrahlung spectrum requires approximately $10^{4}$ particles to simulate a single energetic photon scattered into the backwards direction, which is very inefficient.

To improve upon these efficiencies, a next-event estimator technique is used for the backscatter simulations. These simulations model the backscattered photon spectrum resulting from some defined photon spectrum incident upon a target (or TD). To determine the expected photon spectrum that would be incident upon a radiation detector, the flux at the center of the detector, at point $\mathbf{r}_{\mathbf{d}}$, is determined by the next-event estimator routine. In the simulation, each time a particle (presumably photon or photo-electron) interactions in the target, the probability-weighted flux contribution at point $\mathbf{r}_{\mathbf{d}}$ is calculated. This contribution is given by

$$
\Delta \Phi\left(\mathbf{r}_{\mathbf{d}}\right)=w \frac{p(\boldsymbol{\Omega})}{R^{2}} \exp \left[-\int_{0}^{R} N(s) \sigma(s) d s\right]
$$

where $w$ is the weight of the interacting particle, which may be non-zero if other variance reduction techniques are employed, $p(\boldsymbol{\Omega})$ is the differential probability that the interaction will result in production of a photon directed toward $\mathbf{r}_{\mathbf{d}}, R$ is the distance between the interaction point and $\mathbf{r}_{\mathbf{d}}$, therefore the $1 / R^{2}$ term takes into account the reduction of emanating photon intensity with distance from the interaction point. Finally, the term $\exp \left[-\int_{0}^{R} N(s) \sigma(s) d s\right]$ reduces the contribution to the fluence by the amount proportional to the attenuation of the photon before reaching $\mathbf{r}_{\mathbf{d}} . s$ is the parameterized distance along the line between the interaction point and $\mathbf{r}_{\mathbf{d}}$, and $N(s)$ and $\sigma(s)$ correspond to the atom density, and interaction cross section for photons in the material present at the distance $s$ along the path, respectively.

Although not explicitly contained in equation F.6, the energy of the contribution to the nextevent estimation is at least partially determined by the interaction type and angle. The following examples further explain this. If the interaction that causes the next-event flux estimation is a coherent scatter, the energy at which the next-event flux estimate at $\mathbf{r}_{\mathbf{d}}$ contributes is equal to that of the scattered photon. If the interaction is an incoherent scatter, the angle, $\Omega$ defines the contribution energy by the Kline-Nishina equation. Other interaction types do not constrain the energy when the scattering angle is fixed, such as a bremsstrahlung emission from a slowing electron. In this case the energy of the bremsstrahlung photon is first sampled, then the PDF 
relating the energy of a bremsstrahlung photon to the angle of emission is sampled to determine $p(\boldsymbol{\Omega})$ at that bremsstrahlung photon energy.

Next-event estimators can result in multiple contributions to the calculation of an expected photon spectrum per particle history. For example all three events described in the preceding paragraph could occur when a single photon impinges upon a target. Next-event estimators result in simulations that give statistically convergent and significant results in reasonable calculation times. The following two examples of MCNPX input files indicate the use of next-event estimator tallies (called F5 tallies in MCNPX) for simulating the backscattered photon flux. The first example produced the calculated spectrum shown in Section E.1.11. This input file reflects modifications to the MCNPX code and its data files described in Appendices D and E. Particularly, the line, (plib=.05)calls for the .05P photo-atomic data files, which contain the full set of form factors for Rayleigh scattering, and the .30U photonuclear data files contain NRF resonances and secondary particle information pertaining to NRF $\gamma$ rays.

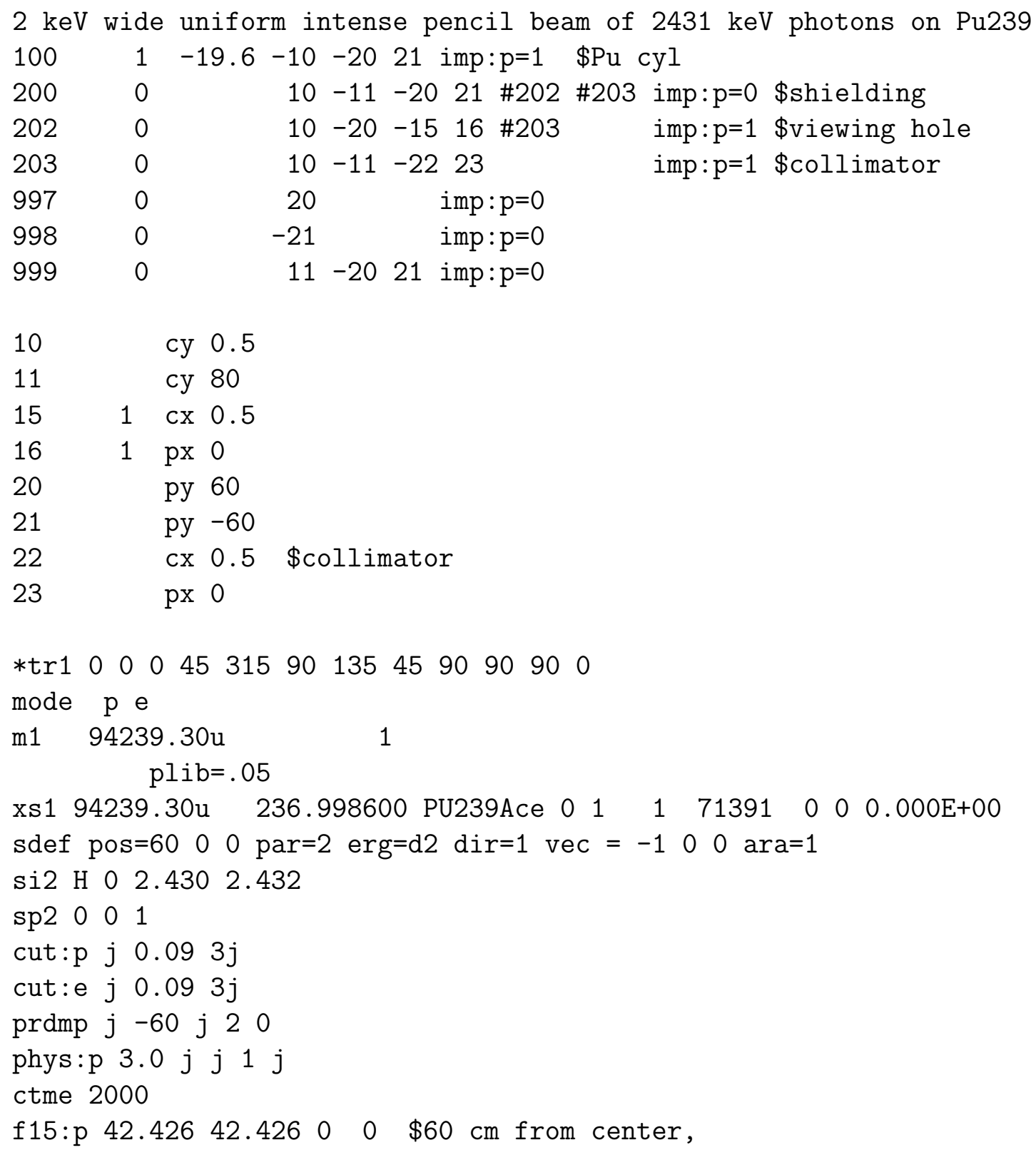


$\begin{array}{lllllllllllllll}\text { e15 } & 0.1 & 39 i & .5 & 509 & 513 & .6 & 14 i & 2.1 & 31 \text { i } & 2.42 & 119 i & 2.43201\end{array}$

A second example explicitly excluded the .05P and .30u data libraries so that the elastic scattering contribution to the backscattered photon spectrum could be calculated using elastic cross section values that also included nuclear Thomson and Delbrück scattering. This calculation was combined with a similar calculation in which the minimum simulated energy was $3 \mathrm{MeV}$ to estimate the inelastic scattering contribution to the background for measurement of ${ }^{137} \mathrm{Ba}$ content in aged ${ }^{137} \mathrm{Cs}$ sources. The cutoff of $3 \mathrm{MeV}$ was used in the following simulation to provide lower uncertainties in the upper energy bins.

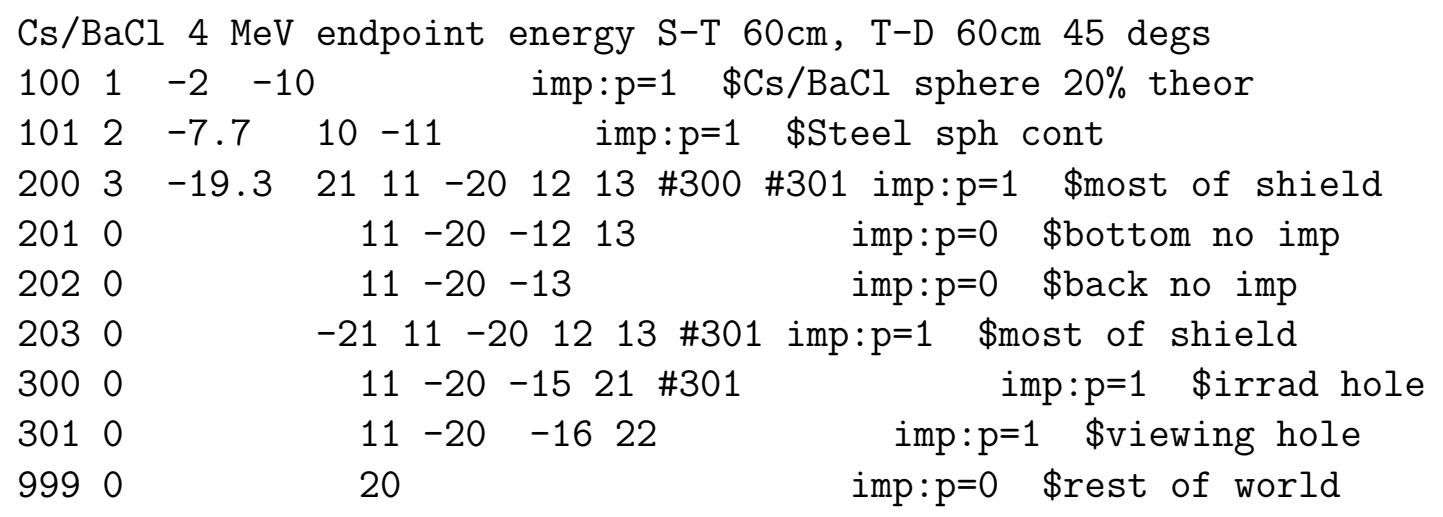

$\begin{array}{llll}10 & & \text { so } 1.742 \\ 11 & & \text { so } & 2.042 \\ 12 & & \text { pz } & -15 \\ 13 & & \text { py } & -15 \\ 15 & & \text { cx } & 1.742 \\ 16 & 1 & \text { cx } & 1.742 \\ 20 & & \text { so } & 65 \\ 21 & & \text { px } & 0 \\ 22 & 1 & \text { px } & 0\end{array}$

*tr1 $0000453159013545909090 \quad 0$

mode $\mathrm{p}$

fcl:p $-106 r$

m1 $17000 \quad 0.5 \quad 55000.25 \quad 56000.25 \quad \$ C s 1 B a 1 C l 2$

m2 $26000 \quad 0.72 \quad 24000 \quad 18 \quad 28000 \quad .10$

m3 740001

dd0 00

print

sdef pos=60 00 par=2 erg=d2 dir=d1 vec $=-100$

si1 H $-1 \quad 9961941$ \$0-5 degrees

sp1 0001

si2 0.14014

sp2 $03.6636 e-8 \quad 9.1549 e-7 \quad 8.3803 e-6 \quad 5.1766 e-5 \quad 0.00010238$

$\begin{array}{llllll}0.00013271 & 0.0001291 & 0.00012422 & 0.00011573 & 0.0001064\end{array}$

$9.7301 e-5 \quad 8.9228 e-5 \quad 8.0441 e-5 \quad 7.3751 e-5 \quad 6.6967 e-5$ 


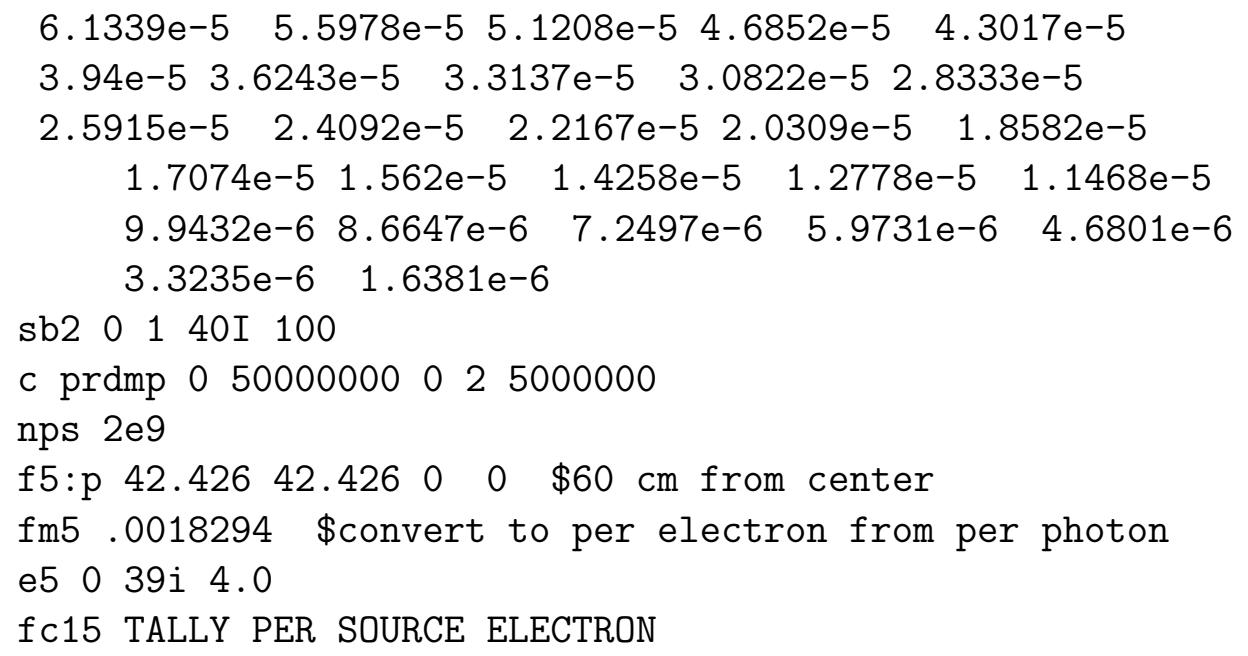

\section{F.4 Detector Response Modeling}

Spectroscopic semiconductor radiation detectors count interactions by measuring a short impulse of electrical current, resulting from the movement of electron-ion pairs in an electric potential. The electron-ion pair is created by interacting particles. The average amount of charge liberated is predicted by the energy deposited by the radiation and the well-studied average energy required to create an ion-pair[61]. The purpose of the MCNPX simulations described in this work is to determine the rate of depositions, and amount of energy deposited, within the detector materials for a given NRF assay geometry. The detector response modeling is accomplished in a very simple manner in MCNPX. The sum energy of simulated particles leaving the detector volume is subtracted from the sum energy of particles entering the volume for each simulated history.

For the detector response calculations used in this report, incident photon spectra were calculated by separate MCNPX simulations, such as those described in Sections F.2 and F.3. These simulations provide calculated spectra at a point or passing through a plane in the modeled space. The detector response model simulations assume that these calculated photon spectra may be represented as though they were uniformly-intense and normally incident upon a plane representing an outer surface of a shielded detector geometry. To further simplify the calculations, monoenergetic photons were simulated as incident upon the shielded geometry and results were superposed to determine the expected detector response to more complicated spectra.

\section{F.4.1 Model Geometry}

Monoenergetic photons were emitted uniformly over a $12 \mathrm{~cm}$ radius disc in the (y,z) plane and irradiated the shielded geometry shown in Figure F.4. The photons are incident from the bottom of the figure. The shielded detector geometry consists of lead of variable thickness, $x_{f}$, in front of a coaxial germanium crystal in an aluminum housing, contained in a square iron enclosure that is open on its front and back. Lead shielding of at least $5 \mathrm{~cm}$ thickness is also present on the sides surrounding the iron enclosure. The geometry of the simulated shielded coaxial HPGe detector is shown in Figure F.4 with dimensions indicated in Table F.2.

The input file for the simulation of the detector response to $3.761 \mathrm{MeV}$ photons for $x_{f}=13.2$ $\mathrm{cm}$ is shown below. 


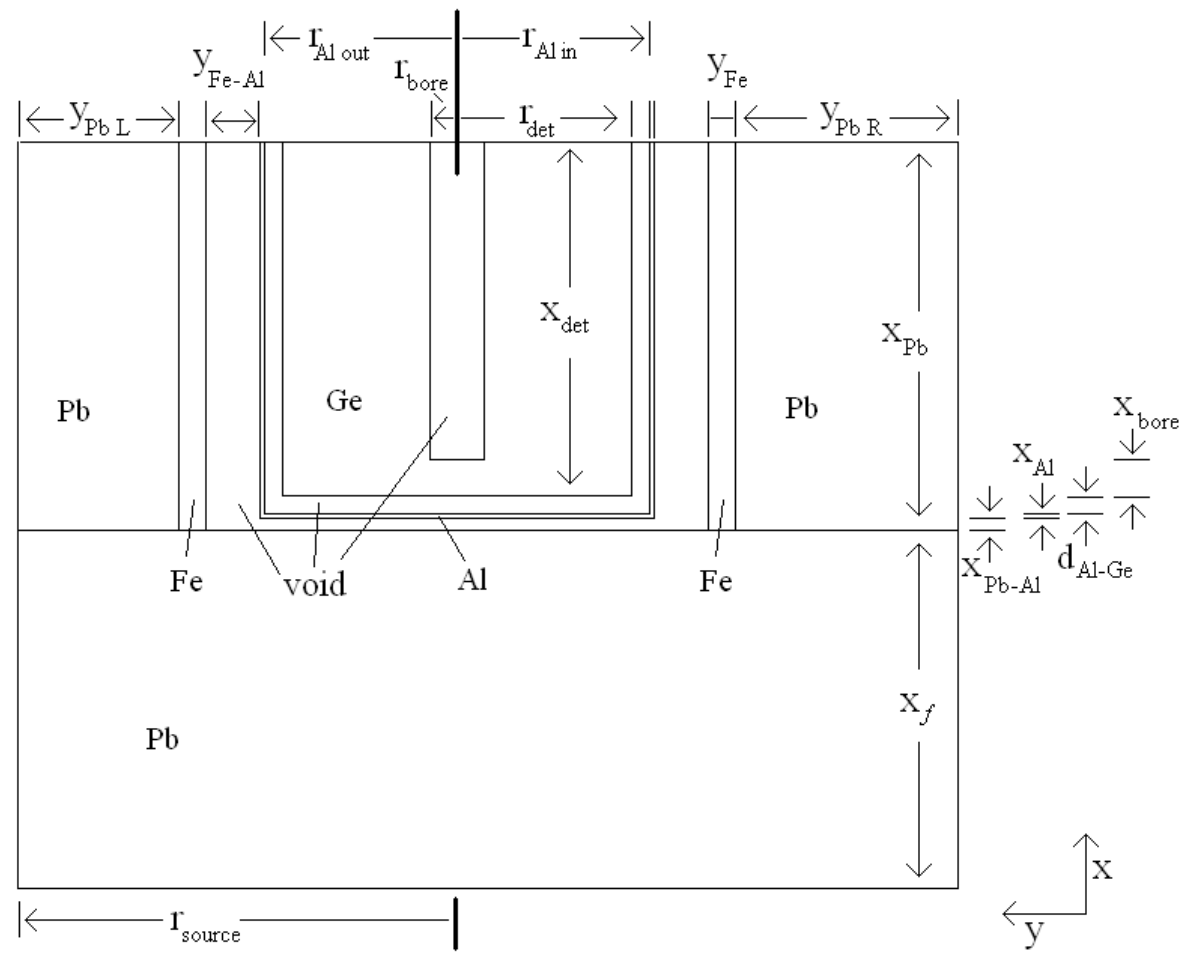

Figure F.4 Horizontal cut-through of the geometry used in MCNPX simulations to estimate photon energy depositions within a shielded HPGe detector. Photons enter the geometry from the bottom, uniformly distributed on the (y,z) plane within a radius, $r_{\text {source }}$, from the germanium crystal center. Dimensions are given in Table F.2. Dimensions given as $r$ indicate radii, and that the object is circular in the perpendicular plane. The HPGe detector is assumed to be set inside an Fe enclosure. The Fe is modeled as a hollow, open-sided rectangular parallelepiped. Below the $\mathrm{Fe}$ is additional $\mathrm{Pb}$ shielding. 
Table F.2 Dimensions of shielded HPGe detector geometry shown in Figure F.4. The final two dimensions listed are not indicated in the figure. $z_{\mathrm{Fe}-\mathrm{Al}}$ is the distance between the top of the $\mathrm{Al}$ case and the top, inner, surface of the Fe enclosure. The dimension, $z_{\mathrm{PbBottom}}$ indicates thickness of $\mathrm{Pb}$ beneath the Fe.

\begin{tabular}{crl}
\hline \hline dimension & \multicolumn{2}{c}{ value } \\
\hline$y_{\mathrm{PbL}}$ & 5 & $\mathrm{~cm}$ \\
$y_{\mathrm{Fe}-\mathrm{Al}}$ & $\geq 1.21$ & $\mathrm{~cm}$ \\
$r_{\text {bore }}$ & 6.2 & $\mathrm{~mm}$ \\
$r_{\text {det }}$ & 3.975 & $\mathrm{~mm}$ \\
$r_{\mathrm{Alin}}$ & 4.375 & $\mathrm{~mm}$ \\
$r_{\mathrm{Alout}}$ & 4.505 & $\mathrm{~mm}$ \\
$y_{\mathrm{Fe}}$ & 1.27 & $\mathrm{~cm}$ \\
$y_{\mathrm{PbR}}$ & 8 & $\mathrm{~cm}$ \\
$x_{\mathrm{det}}$ & 8.05 & $\mathrm{~cm}$ \\
$x_{\mathrm{Pb}}$ & 8.85 & $\mathrm{~cm}$ \\
$x_{\mathrm{Pb}-\mathrm{Al}}$ & 3 & $\mathrm{~mm}$ \\
$x_{\mathrm{Al}}$ & 1 & $\mathrm{~mm}$ \\
$d_{\mathrm{Al}-\mathrm{Ge}}$ & 4 & $\mathrm{~mm}$ \\
$x_{\text {bore }}$ & 8.2 & $\mathrm{~mm}$ \\
$x_{f}$ & variable \\
$r_{\text {source }}$ & 12 & $\mathrm{~cm}$ \\
$z_{\mathrm{Fe}-\mathrm{Al}}$ & $\geq 2.42$ & $\mathrm{~cm}$ \\
$z_{\mathrm{PbBottom}}$ & 7.5 & $\mathrm{~cm}$ \\
\hline
\end{tabular}

$3761 \mathrm{keV}$ photons penetrating $13.2 \mathrm{~cm}$ pb shielding to $100 \% \mathrm{r}$.e. HPGe

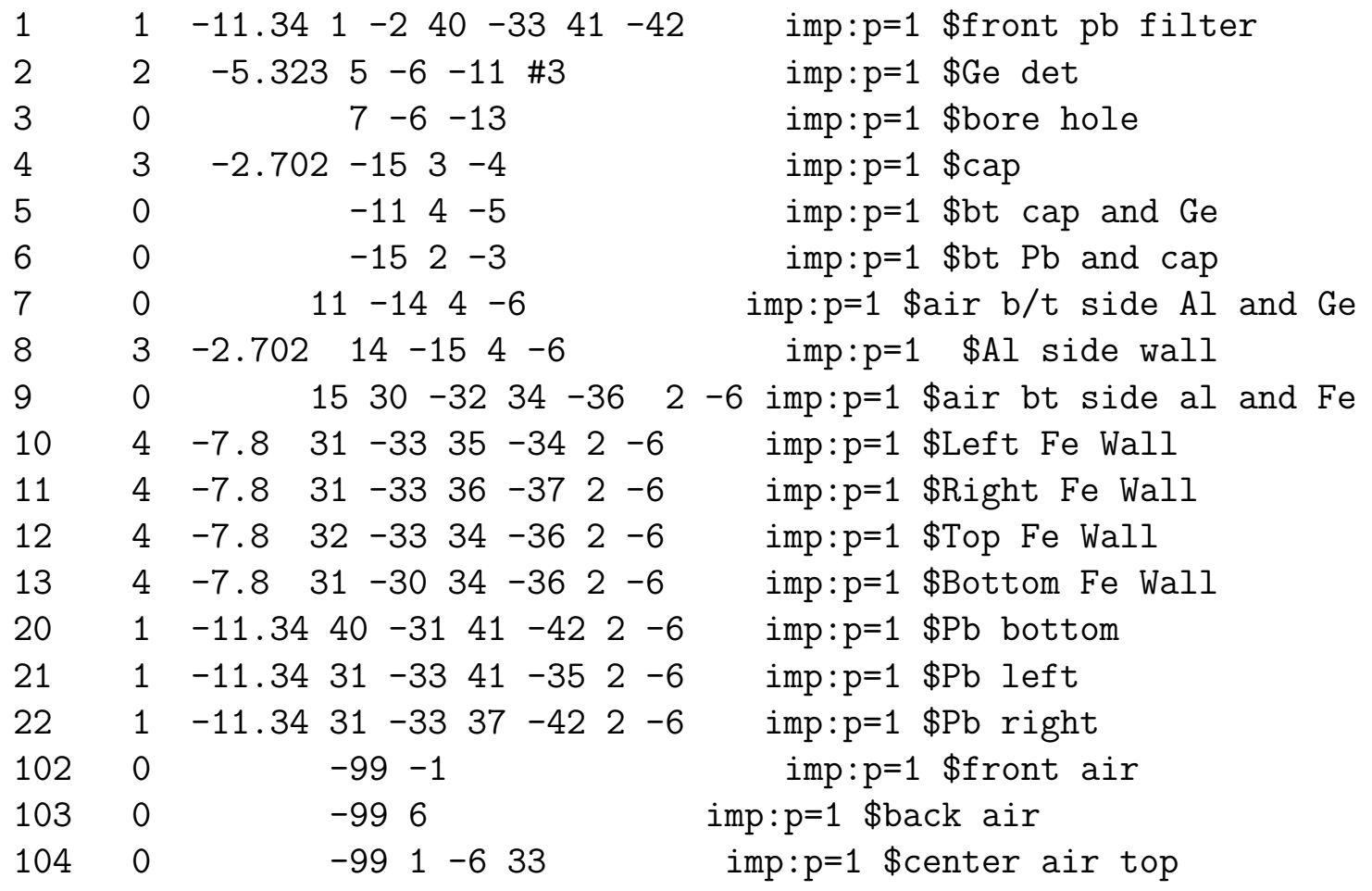




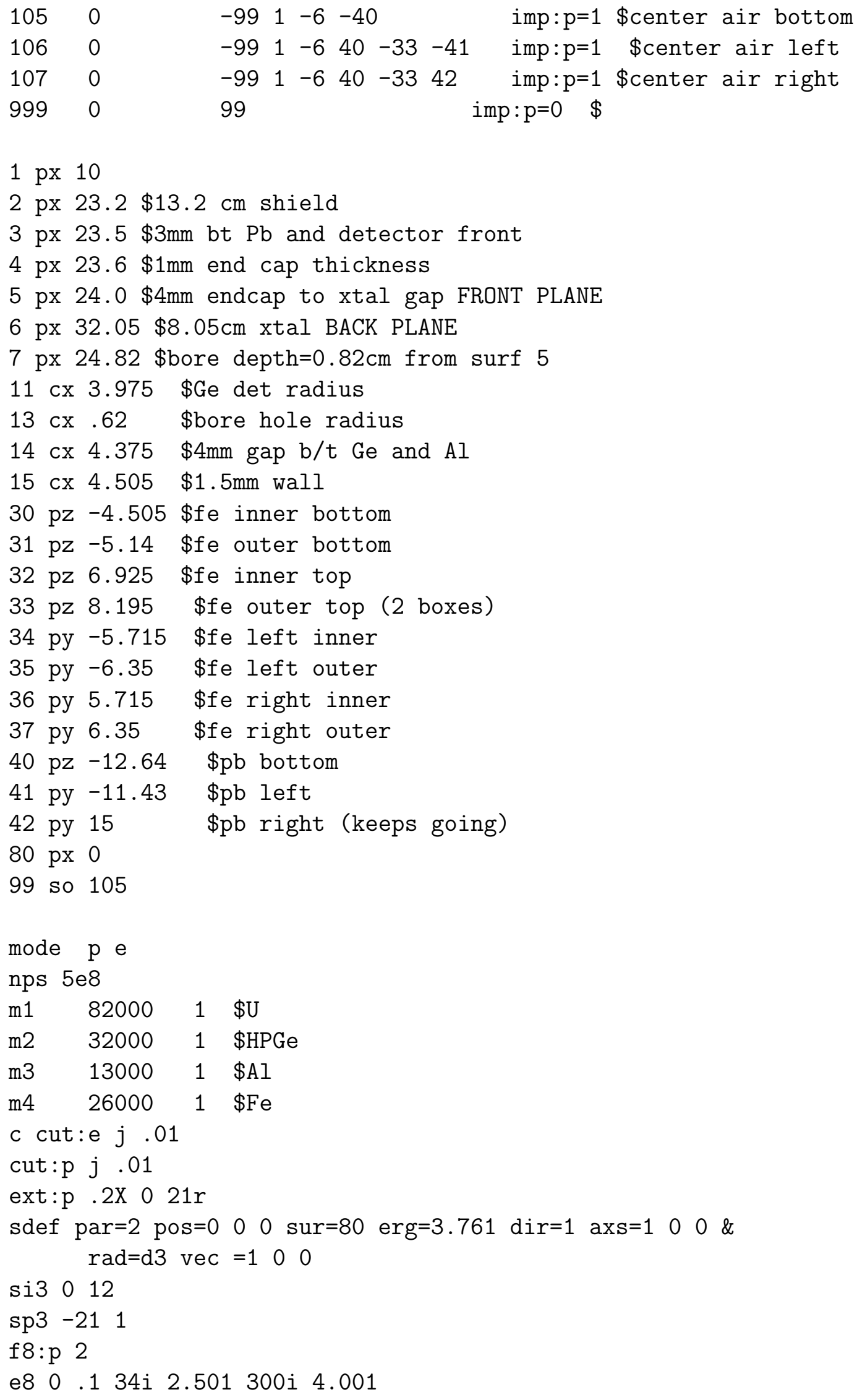

$1 \mathrm{px} 10$

$2 \mathrm{px} 23.2 \$ 13.2 \mathrm{~cm}$ shield

$3 \mathrm{px} 23.5 \$ 3 \mathrm{~mm}$ bt $\mathrm{Pb}$ and detector front

$4 \mathrm{px} 23.6 \$ 1 \mathrm{~mm}$ end cap thickness

$5 \mathrm{px} 24.0 \$ 4 \mathrm{~mm}$ endcap to xtal gap FRONT PLANE

$6 \mathrm{px} 32.05 \$ 8.05 \mathrm{~cm}$ xtal BACK PLANE

7 px 24.82 \$bore depth=0.82cm from surf 5

$11 \mathrm{cx} 3.975$ \$Ge det radius

$13 \mathrm{cx} .62$ \$bore hole radius

$14 \mathrm{cx} 4.375$ \$4mm gap b/t Ge and Al

$15 \mathrm{cx} 4.505 \$ 1.5 \mathrm{~mm}$ wall

$30 \mathrm{pz}-4.505$ \$fe inner bottom

$31 \mathrm{pz}-5.14$ \$fe outer bottom

$32 \mathrm{pz} 6.925$ \$fe inner top

$33 \mathrm{pz} 8.195$ \$fe outer top (2 boxes)

34 py -5.715 \$fe left inner

35 py -6.35 \$fe left outer

36 py 5.715 \$fe right inner

37 py 6.35 \$fe right outer

$40 \mathrm{pz}-12.64 \quad \$ \mathrm{pb}$ bottom

41 py $-11.43 \quad \$ p b$ left

42 py $15 \quad$ \$pb right (keeps going)

$80 \mathrm{px} 0$

99 so 105

mode $\mathrm{p} e$

nps 5 e 8

$\begin{array}{llll}\text { m1 } & 82000 & 1 & \$ \mathrm{U} \\ \mathrm{m} 2 & 32000 & 1 & \$ \mathrm{HPGe} \\ \mathrm{m} 3 & 13000 & 1 & \$ \mathrm{Al} \\ \mathrm{m} 4 & 26000 & 1 & \$ \mathrm{Fe}\end{array}$

c cut:e j .01

cut:p $j .01$

ext:p .2X 0 21r

sdef par=2 pos=0 00 sur=80 erg=3.761 dir=1 axs=1 00 \& $\mathrm{rad}=\mathrm{d} 3 \mathrm{vec}=1 \quad 0 \quad 0$

si3 012

sp3 -211

f8:p 2

$\begin{array}{lllllll}\text { e8 } & 0 & .1 & 34 i & 2.501 & 300 i & 4.001\end{array}$ 


\section{F.5 Electron Stopping Treatment in MCNP}

The process of bremsstrahlung production is complicated by the details of energetic electron transport in materials. Because electrons are charged, they are subjected to the Coulomb force, which has an infinite range. Thus, rather than describing single discrete interactions (as is considered in neutral-particle transport), electrons are treated statistically in the electron transport code, ETRAN[62]. MCNP's electron transport mimics this approach[48]. The energy loss per unit length along the trajectory of an electron is described as a distribution function[63] whose mean is the nominal stopping power of the material[64]. Likewise, the angular deflection of the electron (i.e., $\theta_{12}$ ), and the production of bremsstrahlung are also treated as probability distribution functions[65].

To reduce computational requirements, the electron transport probability distributions are calculated for an energy group whose energy boundaries are given iteratively by

$$
E_{n+1}=\frac{E_{n}}{2^{1 / 8}}
$$

where $E_{1}$ is the maximum electron energy expected in the problem, which is set by default to $100 \mathrm{MeV}$, but is recommended to be changed by the user. The mean distance that an electron travels during energy loss from $E_{n}$ to $E_{n+1}$ is called a step. Each step is further divided into $m$ substeps. There is an empirically predefined default $m$ for each element in MCNP, increasing with $Z$ from 2 to 15 , and the value can be increased above the default through user input. At each substep, the emission of bremsstrahlung, non-radiative energy loss, and angular deflection of the electron are randomly sampled, and the energy and direction of the electron are updated. When the electron energy decreases outside the step, distributions corresponding to the next step are calculated and the process is repeated. This results - on average - in approximately the correct treatment of electron stopping and bremsstrahlung production, however correlations between sampled distributions are ignored. Also, if materials are thinner than a step, inaccuracies may result because the actual electron transport is too coarsely discretized during the sampling of only a few substeps.

There is an additional way in which MCNP can determine the energy group for an electron. This is referred to as the nearest-boundary method, where the step parameters are recalculated when the electron energy becomes closer to $E_{n+1}$ than $E_{n}$. The differences between the two methods become more significant when predicting bremsstrahlung from lower-energy electrons than are of interest here.

\section{F.6 Thick-Target Bremsstrahlung Model}

An additional feature in MCNPX is the thick-target bremsstrahlung model. The code allows the user to select which types of particles are transported during the simulation. When photon transport is turned on (as must be the case in all simulations of interest for NRF), electron transport is not turned on by default. This is primarily due to the hundredfold increase in computation time required to include electron transport. With the exception of the following example, all computational results in this work include electron transport. 


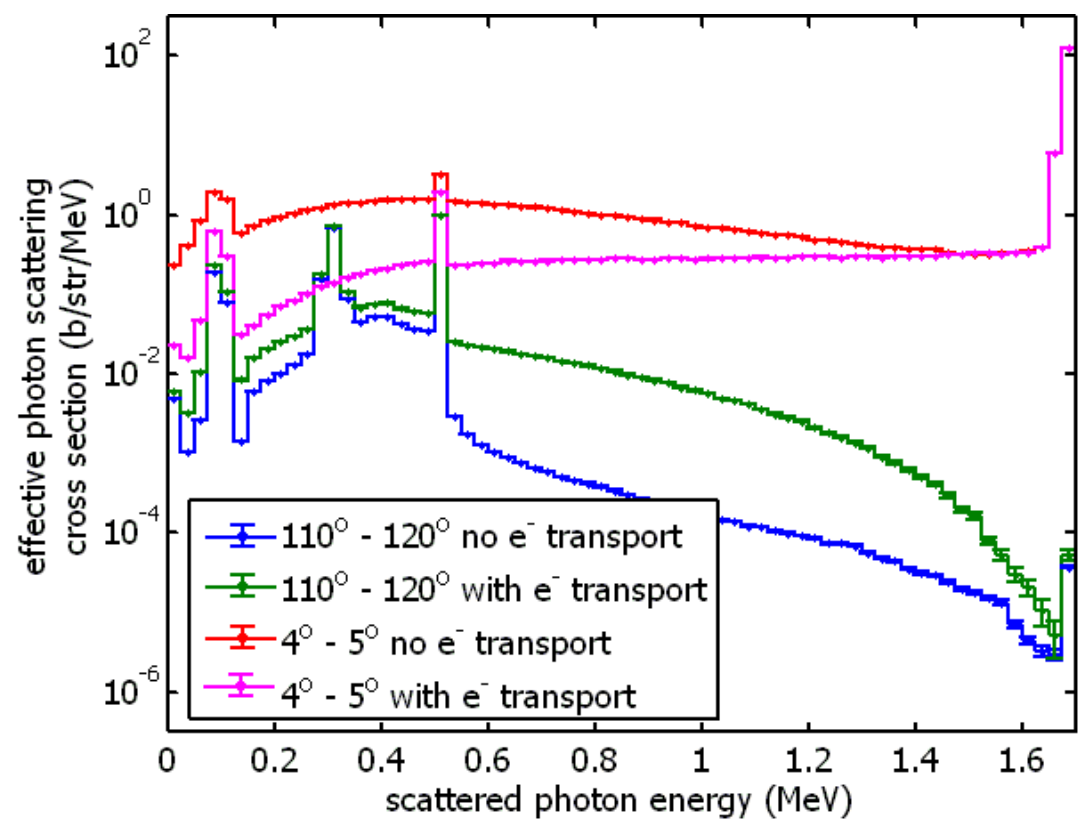

Figure F.5 MCNPX-simulated differential cross section for scattering of $1.7 \mathrm{MeV}$ photons from $1 \mathrm{~cm}$ uranium with and without full electron transport enabled.

When electron transport is not included, a model for bremsstrahlung production is applied whenever an electron would be created during photon transport. The model, called the thick-target bremsstrahlung (TTB) model, immediately produces photons sampled from a bremsstrahlung spectrum at the point of electron creation. The number and direction of the photons are sampled from a tabulated probability distribution that assumes the electron loses all its energy while stopping in a thick target. This assumption is generally quite good. However, when photo-electrons are produced within the electron's range of a material surface, the TTB model can over-predict bremsstrahlung production. Furthermore, the model appears to under sample the emission of bremsstrahlung at large angles relative to the trajectory of the electron-producing photon. The spectra of monoenergetic $1.7 \mathrm{MeV}$ photons normally incident upon the circular surface of a uranium cylinder of $1 \mathrm{~cm}$ radius and $1 \mathrm{~cm}$ thick, as calculated by MCNPX, are shown in Figure F.5. The effective photon scattering cross sections are shown for the angular ranges $4^{\circ}-5^{\circ}$ and $100^{\circ}-$ $110^{\circ}$, with and without electron transport. The TTB model appears to overestimate low-energy bremsstrahlung emission in forwards directions and significantly underestimate bremsstrahlung production in backwards directions. 


\section{Bibliography}

[1] S.J. Tobin, W.S. Charlton, M.H. Ehinger, M.L. Fensin, A.S. Hoover, H.O. Menlove, B.J. Quiter, A. Rajasingam, N.P. Sandoval, S.F. Saavedra, D. Strohmeyer, M.T. Swinhoe, and S.J. Thompson, "Determining Plutonium in Spent Fuel with Nondestructive Assay Techniques," Proceedings of 31st Annual Meeting of ESARDA, Vilnius, Lithuania (2009)

[2] S.J. Tobin, "Determination of Plutonium Content in Spent Fuel with NDA - Why an Integrated Approach?," INMM, July 13-17, 2008, Nashville, TN.(LA-UR-08-03763).

[3] F.R. Metzger, "Resonance Fluorescence in Nuclei," Prog. in Nuc. Phys. 7, (1959) 54.

[4] U. Kneissl, H.H. Pitz, and A. Zilges, "Investigation of Nuclear Structure by Resonance Fluorescence Scattering," Prog. Part. Nucl. Phys. 37, (1996) pp. 349-433.

[5] J.F. Pelowitz (ed.), "MCNPX ${ }^{\mathrm{TM}}$ USERS MANUAL Version 2.6.0," LA-CP-07-1473 (2008).

[6] J.M. Blatt and V.F. Weisskopf, "Theoretical Nuclear Physics," Chapter 12, John Wiley \& Sons, Inc. New York (1952).

[7] D.R. Hamilton, "On Direction Correlation of Successive Quanta," Phys. Rev. 58, (1950) 122.

[8] W.E. Lamb, Jr., "Capture of Neutrons by Atoms in a Crystal," Phys. Rev. 55, (1939) 190.

[9] J.R. Phillips, "Irradiated Fuel Measurements," in Passive Nondestructive Assay of Nuclear Materials, D.T. Reilly, editor. Office of Nuclear Regulatory Research, NUREG/CR-5550. LA-UR-90-732 (1990) 556.

[10] W.B. Wilson, T.R. England, M. Herman, R.E. MacFarlane, and D.W. Muir, "CINDER'90 code for transmutation calculations," Nuclear Data for Science and Technology Conference, Trieste, Italy (1997).

[11] M. Abs, Y. Jongen, E. Poncelet, J.-L. Bol, "The IBA rhodotron TT1000:a very high power E-beam accelerator," Radiation Physics and Chemistry Vol. 71, (2004) 285-288.

[12] J. Pottier, "A new type of RF electron accelerator: the rhodotron," Nucl. Instr. Meth. B40/41 (1989) pp. 943-945.

[13] HIGS flux performance table, DFELL/TUNL, Feb. 5, 2010 (Version 1.0). Accessed online 2, April 2010. http://www.tunl.duke.edu/pdfs/HIGSPerformance.pdf

[14] F.V. Hartemann, S.G. Anderson, C.P.J Barty, D.J. Gibson, C.A. Hagmann, M.S. Johnson, I. Jovanovic, D.P. MaNabb, M.J. Messerly, J.A. Pruet, M.Y. Shverdin, C.W. SIders, and A.M. Tremaine, "Gamma-ray compton light source development at LLNL," Proceedings of PAC07, Albuquerque, New Mexico, (2007) 1245.

[15] H. Ohgaki, T. Noguchi, S. Sugiyama, T. Yamazaki, T. Mikado, M. Chiwaki, K. Yamada, R. Suzuki, and N. Sei, "Linearly polarized photons from Compton backscattering of laser light for nuclear resonance fluorescence experiments," Nuclear Instruments and Methods in Physics Research A 353, (1994) pp. 384-388.

[16] F. Albert, S.G. Anderson, G.A. Anderson, S.M. Betts, D.J. Gibson, C.A. Hagmann, J. Hall, M.S. Johnson, M.J. Messerly, V.A. Semenov, M.Y. Shverdin, A.M. Tremaine, F.V. Hartemann, C.W. Siders, D.P. McNabb, and C.P.J Barty, "Isotope-specific detection of low-density materials with laser-based monoenergetic gammarays," Optics Letters 35, No. 3, (2010) 354.

[17] F.V. Hartemann, F. Albert, G.G. Anderson, S.G. Anderson, A.J. Bayramian, S.M. Betts, T.S. Chu, R.R. Cross, C.A. Ebbers, S.E. Fisher, D.J. Gibson, A.S. Ladran, M.J. Messerly, V.A. Semenov, M.Y. Shverdin, C.W. Siders, D.P. McNabb, C.P.J Barty, A.E. Vlieks, E.N. Jongewaard, and S.G. Tantawi, "Development of a precision tunable gamma-ray source driven by a compact x-band linac," Proceedings of PAC09, Vancouver, British Columbia, (2009) WE5RFP030. 
[18] M.F. Cunningham, J.N. Ullom, T. Miyazaki, S.E. Labov, J. Clarke, T.M. Lanting, A.T. Lee, P.L. Richards, J. Yoond, and H. Spieler, "High-resolution operation of frequency-multiplexed transition-edge photon sensors," Appl. Phys. Letters 81, No. 1 (2002) 159.

[19] J.N. Ullom, J.A. Beall, W.B. Doriese, W.D. Duncan, L. Ferreira, G.C. Hilton, K.D. Irwin, C.D. Reintsema, and L. R. Vale, "Optimized transition-edge x-ray microcalorimeter with $2.4 \mathrm{eV}$ energy resolution at $5.9 \mathrm{keV}$," Appl. Phys. Letters 87, (2005) 194103.

[20] D.T. Vo and P.A. Russo, "Comparisons of the Portable Digital Spectrometer," Los Alamos National Laboratory Report LA-13895-MS (2002).

[21] "Ortec-Lanthanum Bromide Scintillation Detectors Data Sheet", Available online at http://www.orteconline.com/download.asbx?AttributeFileId=e0a47196-05ea-4e35-a5a7-7ab39c66f6e1 Accessed 28 Oct 2010.

[22] J.H. Atkinson and V. Perez-Mendez, "Gas Cerenkov Counters," Rev. of Sci. Inst. 30 (1959) 864.

[23] B.D. Sowerby, "Cerenkov Detectors for Low-energy Gamma-Rays," NIM 97 (1971) pp. 145-149.

[24] D.T. Vo and P.A. Russo, "Comparisons of the Portable Digital Spectrometer," Los Alamos National Laboratory Report LA-13895-MS (2002).

[25] M.J. Berger, J.H. Hubbell, S.M. Seltzer, J. Chang, J.S. Coursey, R. Sukumar, and D.S. Zucker, "XCOM: Photon Cross Sections Database, NIST Standard Reference Database 8 (XGAM)" available online at http://physics.nist.gov/PhysRefData/Xcom/Text/XCOM.html (1998).

[26] W. Bertozzi, J.A. Caggiano, W.K. Hensley, M.S. Johnson, S.E. Korbly, R.J. Ledoux, D.P. McNabb, E.B. Norman, W.H. Park, and G.A. Warren, "Nuclear resononce fluorescence excitations near $2 \mathrm{MeV}$ in ${ }^{235} \mathrm{U}$ and ${ }^{239} \mathrm{Pu}$," Phys. Rev. C 041601(R) (2008).

[27] R.D. Heil, H.H. Pitz, U.E.P Berg, U. Kneissl, K.D. Hummel, G. Kilgus, D. Bohle, A. Richter, C. Wesselborg, and P. Von Brentano, "Observation of orbital magnetic dipole strength in the actinide nuclei ${ }^{232} \mathrm{Th}$ and ${ }^{238} \mathrm{U} . "$ Nuc. Phys. A476 (1988) pp. 39-47

[28] A. Zilges, P. von Brentano, R.-D. Herzberg, U. Kneissl, J. Margraf, H. Maser, N. Pietralla, and H.H. Pitz, "Srong dipole excitations around $1.8 \mathrm{MeV}$ in ${ }^{238} \mathrm{U}$," Phys. Rev. C 52, No. 2 (1995) R468.

[29] M.B. Chadwick, P. Obloinsk, M. Herman, N.M. Greene, R.D. McKnight, D.L. Smith, P.G. Young, R.E. MacFarlane, G.M. Hale, S.C. Frankle, A.C. Kahler, T. Kawano, R.C. Little, D.G. Madland, P. Moller, R.D. Mosteller, P.R. Page, P. Talou, H. Trellue, M.C. White, et al. "ENDF/B-VII.0: Next Generation Evaluated Nuclear Data Library for Nuclear Science and Technology," Nuclear Data Sheets Volume 107, Issue 12, (December 2006) pp. 2931-3118.

[30] R. E. MacFarlane, "NJOY 99 Nuclear Data Processing System", code available at http://t2.lanl.gov/codes/njoy99/.

[31] E. Browne, "Nuclear Data Sheets for A = 235,239," Nuclear Data Sheets 98, (2003) pp. 665-800.

[32] G.A. Warren, J.A. Caggiano, E.A. Miller, W. Bertozzi, A. Klimenko, S.E. Korbly, R.J. Ledoux, and W.H. Park, "Nuclear Resonance Fluorescence of ${ }^{235} \mathrm{U}$ above $3 \mathrm{MeV}$," 2007 IEEE Nuclear Science Symposium Conference Record. 2047.

[33] J.L. McFarland, M.S. Thesis, "A technological step towards threat reduction: nuclear resonance fluorescence." Department of Nuclear Engineering, University of California, Berkeley. (2009).

[34] W. Geiger, Zs. Németh, I. Bauske, P. von Brantano, R.D. Heil, R.-D. Herzberg, U. Kneissl, J. Margraf, H. Maser, N. Pietralla, H.H. Pitz, C. Wesselborg, A. Zilges, "Low-lying dipole excitations in the isotopes ${ }^{113,114}$ Cd," Nuc. Phys. A 580, (1994) 263-276.

[35] M. Scheck, H. von Garrel, N. Tsoneva, D. Belic, P. von Brentano, C. Fransen, A. Gade, J. Jolie, U. Kneissl, C. Kohstall, A. Linnemann, A. Nord, N. Pietralla, H.H. Pitz, F. Stedile, C. Stoyanov, and V. Werner, "Dipole strength distributions in the stable $\mathrm{Ba}$ isotopes ${ }^{134-138} \mathrm{Ba}$ : A study in the mass region of a nuclear shape transition." Phys. Rev. C 70044319 (2004). 
[36] I. Bauske, J.M. Arias, P. von Brentano, A. Frank, H. Friedrichs, R.D. Heil, R.-D. Herzberg, F. Hoyler, P. Van Isacker, U. Kneissl, J. Margraf, H.H. Pitz, C. Wesselborg, and A. Zilges, "First Observation of Scissors Mode States in an Odd-Mass Nuclueus," Physical Review Letters 71 (1993) 975.

[37] H. Burkhardt, P. Koehler, R. Rietmuller, B.H. Wiik, R. Fohrmann, J. Franzke, H. Krasemann, R. Maschuw, G. Poelz, J. Reichardt, J. Ringel, O Romer, R. Rusch, P. Schmuser, R.van Staa, J. Freeman, P. Lecompte, T. Mayer, S.L Wu, and G. Zobernig, "The Tasso Gas and Aerogel Cherenkov Counters," NIM 184 (1981) 319-331.

[38] Y. Allkofer, C. Amsler, S. Horikawa, I. Johnson, C. Regenfus, J. Rochet "NA novel aerogel Cherenkov detector for DIRAC-II," NIM A 582 (2007) 497-508.

[39] SCALE-4.3, Modular System for Performing Standardized Computer Analyses for Licencing Evaluation. Oak Ridge National Laboratory, 1997. RSICC Computer Code Collection CCC-0545/12.

[40] R.B. Firestone, S.Y.F. Chu, and C.M. Baglin, "1998 Update to the 8th Edition of the Table of Isotopes," CD-ROM, John Wiley \& Sons, Inc. (1998).

[41] J.M. Verbeke, C. Hagmann, D. Wright, "Simulation of Neutron and Gamma Ray Emission from Fission and Photofission," UCRL-AR-228518. LLNL report, May 11, 2010.

[42] C.A. Hagmann, J.M. Hall, M.S. Johnson, D.P.McNabb, J.H. Kelley, C. Huibregtse, E. Kwan, G. Rusev, and A.P. Tonchev, "Transmission-based detection of nuclides with nuclear resonance fluorescence using a quasimonoenergetic photon source," J. App. Phys. 106, 084901 (2009).

[43] R. Vodhanel, M.K. Brussel, R. Moreh, W.C. Sellyey, and T.E. Chapuran, "Strong M1 transitions in ${ }^{23} \mathrm{Na}$ below 10 MeV," Phys. Rev. C 292 (1984) 409.

[44] L.P. Ekström and R.B. Firestone, "WWW Table of Radioactive Isotopes," database version 2/28/99 from URL http://ie.lbl.gov/toi/index.htm

[45] J.S. Hendricks and B.J. Quiter, "MCNP/X Form Factor Upgrade for Improved Photon Transport," (LA-UR10-01096) Journal of Nuclear Technology, in Press.

[46] G.W. McKinney, A.B. McKinney, J.S. Hendricks, D.B. Pelowitz, B.J. Quiter, MCNPX NRF Library - Release 2, Proc. ANS Ann. Mtg. (San Diego, CA July 13-17, 2010).

[47] J.E. Sweezy, et. al, "MCNP A General Monte Carlo N-Particle Transport Code, Version 5," LA-UR-03-1987, (2003, revised 2005).

[48] J.F. Briesmeister (ed.), "MCNP ${ }^{\mathrm{TM}}$ A General Monte Carlo NParticle Transport Code Version 4C," LA13709M, (2000).

[49] D.E. Cullen, J.H. Hubbell, and L. Kissel, "EPDL97: the evaluated photon data library, '97 version," UCRL50400, Vol. 6, Rev. 5 (1997).

[50] E.D. Cashwell et. al. "Monte Carlo Photon Codes: MCG and MCP" LA-5157-MS, (March 1973).

[51] B.J. Quiter, B.A. Ludewigt, V.V. Mozin, and S.J. Tobin, "Nondestructive Spent Fuel Assay Using Nuclear Resonance Fluorescence," INMM, July 12-16, 2009, Tucson, AZ.

[52] Lynn Kissel, "RTAB: the Rayleigh scattering database," Radiation Physics and Chemistry Vol. 59, (2000) $185-200$.

[53] C.J. Lodwick and H.J. Spitz, "Modification to the Monte Carlo N-Particle Code for Simulating Direct, In Vivo Measurements of Stable Lead in Bone," Health Physics, Vol. 94, No. 6 (2008) pp. 519-526.

[54] L.S. Waters (ed.), "MCNX ${ }^{\mathrm{TM}}$ USER'S MANUAL Version 2.3.0," LA-UR-02-2607 (2002).

[55] T. Belgya, "Discrete level library files," Reference Input Parameter Library. Available online at http://wwwnds.iaea.org/RIPL-2/ (2002).

[56] Jag Tuli, "Evaluated Nuclear Structure Data File (ENSDF)," Database version of January 20, 2010, Brookhaven National Laboratory. Available online at: http://www.nndc.bnl.gov/ensdf/ (2010). 
[57] Thomson Reuters, "INSPEC," Available online at http://thomsonreuters.com/products_services/science/ science_products/a-z/inspec (2009).

[58] Redhat, "Cygwin," Datasheet available online at http://www.redhat.com/services/custom/cygwin (2008).

[59] "ENDF-6 Formats Manual," ed. M. Herman. Document ENDF-102, BNL-NCS-44945-05-Rev (2001).

[60] M.L. Tobias, D.R. Vondy, and M.P. Lietzke, "NIGHTMARE - An IBM 7090 Code for the Calculation of Gamma Heating in Cylindrical Geometry," USAEC Report ORNL-3198, Oak Ridge National Laboratory, Feb. 26, (1972).

[61] G.F. Knoll, "Radiation detection and measurement," John Wiley \& Sons Inc. New York, (2000)

[62] S.M. Seltzer, "An Overview of ETRAN Monte Carlo Methods," Ch. 7 of Monte Carlo Transport of Electrons and Photons, edited by T.M Jenkins, W.R Nelson, and A. Rindi, Plenum Press (1988).

[63] L. Landau, "On the Energy Loss of Fast Particles by Ionization, J. Phys. USSR 8 (1944).

[64] M. J. Berger, "Monte Carlo Calculation of the Penetration and Diffusion of Fast Charged Particles, in Methods in Computational Physics, Vol. 1, edited by B. Alder, S. Fernbach, and M. Rotenberg, Academic Press, New York, (1963) 135.

[65] S. Goudsmit and J. L. Saunderson, "Multiple Scattering of Electrons, Phys. Rev. 57 (1940) 24.

[66] G.A. Warren, et al., "Nuclear Resonance Fluorescence of ${ }^{235}$ U," 2006 IEEE Nuclear Science Symposium Conference Record. 914.

[67] M.L. Fensin, S.J. Tobin, N.P. Sandoval, S.J. Thompson, M.T. Swinhoe, "A Monte Carlo Linked Depletion Spent Fuel Library for Assessing Varied Nondestructive Assay Techniques for Nuclear Safeguards," LA-UR 09-01188, ANS Advances in Nuclear Fuel Management IV, Hilton Head Island, SC (2009).

[68] S. Agostinelli, et al. "GEANT4 - a simulation toolkit," NIM A 506, No. 3 (2003) pp. 250-303.

[69] R. Buck and E. Lent, "COG: A New, High-Resolution Code for Modeling Radiation Transport," UCRL-5200093-6, (1993).

[70] J.H. Hubbell, W.J. Weigele, E.A. Briggs, R.T. Brown, D.T. Cromer, and R.J. Howerton, "Atomic Form Factors, Incoherent Scattering Functions, and Photon Scattering Cross Sections," J. Phys. Chem. Ref. Data Vol 4, No. 3 (1975).

[71] F. Salvat, J.M. Fernández-Varea, E. Acosta, and J. Sempau, "PENELOPE A Code System for Monte Carlo Simulation of Electron and Photon Transport," OECD Nuclear Energy Agency, ISBN 92-64-18475-9, (2001).

[72] S.M. Seltzer and M.J. Berger, "Bremsstrahlung spectra from electron interactions with screened atomic nuclei and orbital electonrs," NIM B 12, (1985) pp. 95-134.

[73] C.J. Hagmann and J. Pruet,"Photon production through multi-step processes important in nuclear resonance fluorescence experiments," NIM B 259, (2007) pp. 895-909.

[74] A. Tanskanen, "Assessment of the neutron and gamma sources of the spent BWR fuel". Interim report on Task FIN JNT A 1071 of the Finnish Support Programme to IAEA Safeguards. STUK-YTO-TR 170. Helsinki 2000 .

[75] W.J. Alston III, H.H. Wilson, and E.C. Booth, "Resonance fluorescence and absorption measurements of transition strengths in odd-mass nuclei," Nuc. Phys. A 116, (1968) pp. 281-288.

[76] I.Y. Lee, R.M. Clark, M. Cromaz, M.A. Deleplanque, M. Descovich, R.M. Diamond, P. Fallon, A.O. Macchiavelli, F.S. Stephens, and D. Ward, "GRETINA: A gamma ray energy tracking array," Nuc. Phys. A 746 (2004) pp. 255c-259c. 